RESEARCH-EXPOSITORY SURVEY

\title{
A SURVEY OF \\ THE ADDITIVE EIGENVALUE PROBLEM (WITH APPENDIX BY M. KAPOVICH)
}

\author{
SHRAWAN KUMAR* \\ Department of Mathematics \\ University of North Carolina \\ Chapel Hill, NC 27599-3250, USA \\ shrawan@email.unc.edu
}

\begin{abstract}
The classical Hermitian eigenvalue problem addresses the following question: What are the possible eigenvalues of the sum $A+B$ of two Hermitian matrices $A$ and $B$, provided we fix the eigenvalues of $A$ and $B$. A systematic study of this problem was initiated by H. Weyl (1912). By virtue of contributions from a long list of mathematicians, notably Weyl (1912), Horn (1962), Klyachko (1998) and Knutson-Tao (1999), the problem is finally settled. The solution asserts that the eigenvalues of $A+B$ are given in terms of certain system of linear inequalities in the eigenvalues of $A$ and $B$. These inequalities are given explicitly in terms of certain triples of Schubert classes in the singular cohomology of Grassmannians and the standard cup product. Belkale (2001) gave an optimal set of inequalities for the problem in this case. The Hermitian eigenvalue problem has been extended by Knutson-Tao (2000) and Kapovich-Leeb-Millson (2005) for any semisimple complex algebraic group $G$. Their solution is again in terms of a system of linear inequalities obtained from certain triples of Schubert classes in the singular cohomology of the partial flag varieties $G / P$ ( $P$ being a maximal parabolic subgroup) and the standard cup product. However, their solution is far from being optimal. In a joint work with P. Belkale, we define a deformation of the cup product in the cohomology of $G / P$ and use this new product to generate our system of inequalities which solves the problem for any $G$ optimally (as shown by Ressayre). This article is a survey (with more or less complete proofs) of this additive eigenvalue problem. The eigenvalue problem is equivalent to the saturated tensor product problem. We also give an extension of the saturated tensor product problem to the saturated restriction problem for any pair $G \subset \widehat{G}$ of connected reductive algebraic groups.

In the appendix by M. Kapovich, connections between metric geometry and the representation theory of complex semisimple algebraic groups is explained. The connections run through the theory of buildings. This connection is exploited to give a uniform (though not optimal) saturation factor for any $G$.
\end{abstract}

DOI: $10.1007 / \mathrm{s} 00031$

* Supported by NSF grants.

Received May 21, 2013. Accepted June 18, 2014.

Corresponding Author: Sh. Kumar, e-mail: shrawan@email.unc.edu. 


\section{SHRAWAN KUMAR}

\section{Contents}

1. Introduction

2. Notation

3. Determination of the eigencone/saturated tensor cone

4. Specialization of Theorem 10 to $G=\mathrm{SL}(n)$ : Horn inequalities

5. Deformed product

6. Efficient determination of the eigencone

7. Study of the saturated restriction semigroup and irredundancy of its inequalities

8. Notational generalities on classical groups

9. Comparison of the eigencones under diagram automorphisms

10. Saturation problem

11. Deformed product and Lie algebra cohomology

12. A restricted functoriality of the deformed product and a product formula

13. Tables of the deformed product $\odot$ for the groups of type $B_{2}, G_{2}, B_{3}$ and $\mathrm{C}_{3}$

14. An explicit determination of the eigencone for the ranks 2 and 3 simple Lie algebras

15. Appendix (by M. Kapovich)

16. Bibliography

\section{Introduction}

This is a fairly self-contained survey article on the classical Hermitian eigenvalue problem and its generalization to an arbitrary connected reductive group.

For any $n \times n$ Hermitian matrix $A$, let $\lambda_{A}=\left(\lambda_{1} \geq \cdots \geq \lambda_{n}\right)$ be its set of eigenvalues written in descending order. Recall the following classical problem, known as the Hermitian eigenvalue problem: Given two $n$-tuples of nonincreasing real numbers: $\lambda=\left(\lambda_{1} \geq \cdots \geq \lambda_{n}\right)$ and $\mu=\left(\mu_{1} \geq \cdots \geq \mu_{n}\right)$, determine all possible $\nu=\left(\nu_{1} \geq \cdots \geq \nu_{n}\right)$ such that there exist Hermitian matrices $A, B, C$ with $\lambda_{A}=\lambda, \lambda_{B}=\mu, \lambda_{C}=\nu$ and $C=A+B$. This problem has a long history, starting with the work of Weyl (1912) followed by works of Fan (1949), Lidskii (1950), Wielandt (1955), and culminating in the following conjecture given by Horn (1962).

For any positive integer $r<n$, inductively define the set $S_{r}^{n}$ as the set of triples $(I, J, K)$ of subsets of $[n]:=\{1, \ldots, n\}$ of cardinality $r$ such that

$$
\sum_{i \in I} i+\sum_{j \in J} j=r(r+1) / 2+\sum_{k \in K} k
$$

and for all $0<p<r$ and $(F, G, H) \in S_{p}^{r}$ the following inequality holds:

$$
\sum_{f \in F} i_{f}+\sum_{g \in G} j_{g} \leq p(p+1) / 2+\sum_{h \in H} k_{h} .
$$


Conjecture 1. A triple $\lambda, \mu, \nu$ occurs as eigenvalues of Hermitian $n \times n$ matrices $A, B, C$ respectively such that $C=A+B$ if and only if

$$
\sum_{i=1}^{n} \nu_{i}=\sum_{i=1}^{n} \lambda_{i}+\sum_{i=1}^{n} \mu_{i}
$$

and for all $1 \leq r<n$ and all triples $(I, J, K) \in S_{r}^{n}$, we have

$$
\sum_{k \in K} \nu_{k} \leq \sum_{i \in I} \lambda_{i}+\sum_{j \in J} \mu_{j}
$$

Horn's above conjecture was settled in the affirmative (cf. Corollary 15) by combining the work of Klyachko (1998) with the work of Knutson-Tao (1999) on the 'saturation' problem.

The above system of inequalities is overdetermined. Belkale (2001) proved that a certain subset of the above set of inequalities suffices. Subsequently, KnutsonTao-Woodward (2004) proved that the subsystem of inequalities given by Belkale forms an irredundant system of inequalities.

Now, we discuss a generalization of the above Hermitian eigenvalue problem (which can be rephrased in terms of the special unitary group $\mathrm{SU}(n)$ and its complexified Lie algebra $\mathfrak{s l}(n))$ to an arbitrary complex semisimple group. Let $G$ be a connected, semisimple complex algebraic group. We fix a Borel subgroup $B$, a maximal torus $H$, and a maximal compact subgroup $K$. We denote their Lie algebras by the corresponding Gothic characters: $\mathfrak{g}, \mathfrak{b}, \mathfrak{h}, \mathfrak{k}$ respectively. Let $R^{+}$be the set of positive roots (i.e., the set of roots of $\mathfrak{b}$ ) and let $\Delta=\left\{\alpha_{1}, \ldots, \alpha_{\ell}\right\} \subset R^{+}$ be the set of simple roots. There is a natural homeomorphism $\delta: \mathfrak{k} / K \rightarrow \mathfrak{h}_{+}$, where $K$ acts on $\mathfrak{k}$ by the adjoint representation and $\mathfrak{h}_{+}:=\left\{h \in \mathfrak{h}: \alpha_{i}(h) \geq 0 \forall i\right\}$ is the positive Weyl chamber in $\mathfrak{h}$. The inverse map $\delta^{-1}$ takes any $h \in \mathfrak{h}_{+}$to the $K$-conjugacy class of $\sqrt{ }-1 h$.

For any positive integer $s$, define the eigencone

$$
\bar{\Gamma}_{s}(\mathfrak{g}):=\left\{\left(h_{1}, \ldots, h_{s}\right) \in \mathfrak{h}_{+}^{s} \mid \exists\left(k_{1}, \ldots, k_{s}\right) \in \mathfrak{k}^{s}: \sum_{j=1}^{s} k_{j}=0 \text { and } \delta\left(k_{j}\right)=h_{j} \forall j\right\} .
$$

By virtue of the general convexity result in symplectic geometry (cf. [MFK, Thm. 8.9]; also see the proof of Theorem 5), the subset $\bar{\Gamma}_{s}(\mathfrak{g}) \subset \mathfrak{h}_{+}^{s}$ is a convex rational polyhedral cone (defined by certain inequalities with rational coefficients). The aim of the general additive eigenvalue problem is to find the inequalities describing $\bar{\Gamma}_{s}(\mathfrak{g}$ ) explicitly. (The case $\mathfrak{g}=\mathfrak{s l}(n)$ and $s=3$ specializes to the Hermitian eigenvalue problem if we replace $C$ by $-C$.)

Let $\Lambda=\Lambda(H)$ denote the character group of $H$ and let $\Lambda_{+}:=\left\{\lambda \in \Lambda: \lambda\left(\alpha_{i}^{\vee}\right) \geq\right.$ $0 \forall$ simple coroots $\left.\alpha_{i}^{\vee}\right\}$ denote the set of all the dominant characters. Then, the set of isomorphism classes of irreducible (finite-dimensional) representations of $G$ is parameterized by $\Lambda_{+}$via the highest weights of irreducible representations. For $\lambda \in \Lambda_{+}$, we denote by $V(\lambda)$ the corresponding irreducible representation (of highest weight $\lambda$ ). 


\section{SHRAWAN KUMAR}

Similar to the eigencone $\bar{\Gamma}_{s}(\mathfrak{g})$, one defines the saturated tensor semigroup $\Gamma_{s}(G)=\left\{\left(\lambda_{1}, \ldots, \lambda_{s}\right) \in \Lambda_{+}^{s}:\left[V\left(N \lambda_{1}\right) \otimes \cdots \otimes V\left(N \lambda_{s}\right)\right]^{G} \neq 0\right.$ for some $\left.N \geq 1\right\}$.

Then, under the identification $\varphi: \mathfrak{h} \stackrel{\sim}{\rightarrow} \mathfrak{h}^{*}$ (via the Killing form)

$$
\varphi\left(\bar{\Gamma}_{s}(\mathfrak{g})\right) \cap \Lambda_{+}^{s}=\Gamma_{s}(G)
$$

(cf. Theorem 5).

For any $1 \leq j \leq \ell$, define the element $x_{j} \in \mathfrak{h}$ by

$$
\alpha_{i}\left(x_{j}\right)=\delta_{i, j}, \forall 1 \leq i \leq \ell .
$$

Let $P \supset B$ be a standard parabolic subgroup with Lie algebra $\mathfrak{p}$ and let $\mathfrak{l}$ be its unique Levi component containing the Cartan subalgebra $\mathfrak{h}$. Let $\Delta(P) \subset \Delta$ be the set of simple roots contained in the set of roots of $\mathfrak{l}$. Let $W_{P}$ be the Weyl group of $P$ (which is, by definition, the Weyl Group of the Levi component $L$ ) and let $W^{P}$ be the set of the minimal length representatives in the cosets of $W / W_{P}$. For any $w \in W^{P}$, define the Schubert variety:

$$
X_{w}^{P}:=\overline{B w P / P} \subset G / P .
$$

It is an irreducible (projective) subvariety of $G / P$ of dimension $\ell(w)$. Let $\mu\left(X_{w}^{P}\right)$ denote the fundamental class of $X_{w}^{P}$ considered as an element of the singular homology with integral coefficients $H_{2 \ell(w)}(G / P, \mathbb{Z})$ of $G / P$. Then, from the Bruhat decomposition, the elements $\left\{\mu\left(X_{w}^{P}\right)\right\}_{w \in W^{P}}$ form a $\mathbb{Z}$-basis of $H_{*}(G / P, \mathbb{Z})$. Let $\left\{\left[X_{w}^{P}\right]\right\}_{w \in W^{P}}$ be the Poincaré dual basis of the singular cohomology $H^{*}(G / P, \mathbb{Z})$. Thus, $\left[X_{w}^{P}\right] \in H^{2(\operatorname{dim} G / P-\ell(w))}(G / P, \mathbb{Z})$. Write the standard cup product in $H^{*}(G / P, \mathbb{Z})$ in the $\left\{\left[X_{w}^{P}\right]\right\}$ basis as follows:

$$
\left[X_{u}^{P}\right] \cdot\left[X_{v}^{P}\right]=\sum_{w \in W^{P}} c_{u, v}^{w}\left[X_{w}^{P}\right] .
$$

Introduce the indeterminates $\tau_{i}$ for each $\alpha_{i} \in \Delta \backslash \Delta(P)$ and define a deformed cup product $\odot$ as follows:

$$
\left[X_{u}^{P}\right] \odot\left[X_{v}^{P}\right]=\sum_{w \in W^{P}}\left(\prod_{\alpha_{i} \in \Delta \backslash \Delta(P)} \tau_{i}^{\left(w^{-1} \rho-u^{-1} \rho-v^{-1} \rho-\rho\right)\left(x_{i}\right)}\right) c_{u, v}^{w}\left[X_{w}^{P}\right],
$$

where $\rho$ is the (usual) half sum of positive roots of $\mathfrak{g}$. By Corollary 22 and the identity (31), whenever $c_{u, v}^{w}$ is nonzero, the exponent of $\tau_{i}$ in the above is a nonnegative integer. Moreover, the product $\odot$ is associative (and clearly commutative). The cohomology algebra of $G / P$ obtained by setting each $\tau_{i}=0$ in $\left(H^{*}(G / P, \mathbb{Z}) \otimes \mathbb{Z}\left[\tau_{i}\right], \odot\right)$ is denoted by $\left(H^{*}(G / P, \mathbb{Z}), \odot_{0}\right)$. Thus, as a $\mathbb{Z}$-module, this is the same as the singular cohomology $H^{*}(G / P, \mathbb{Z})$ and under the product $\odot_{0}$ it is associative (and commutative). The definition of the deformed product $\odot_{0}$ (now known as the Belkale-Kumar product) was arrived at from the crucial concept of Levi-movability as in Definition 4. For a cominuscule maximal parabolic $P$, the product $\odot_{0}$ coincides with the standard cup product (cf. Lemma 23).

Now we are ready to state the main result on solution of the eigenvalue problem for any connected semisimple $G$ (cf. Corollaries 11 and 29). For a maximal parabolic $P$, let $\alpha_{i_{P}}$ be the unique simple root not in the Levi of $P$ and let $\omega_{P}:=\omega_{i_{P}}$ be the corresponding fundamental weight. 
Theorem 2. Let $\left(h_{1}, \ldots, h_{s}\right) \in \mathfrak{h}_{+}^{s}$. Then, the following are equivalent:

(a) $\left(h_{1}, \ldots, h_{s}\right) \in \bar{\Gamma}_{s}(\mathfrak{g})$.

(b) For every standard maximal parabolic subgroup $P$ in $G$ and every choice of s-tuples $\left(w_{1}, \ldots, w_{s}\right) \in\left(W^{P}\right)^{s}$ such that

$$
\left[X_{w_{1}}^{P}\right] \cdots\left[X_{w_{s}}^{P}\right]=d\left[X_{e}^{P}\right] \text { for some } d \neq 0,
$$

the following inequality holds:

$$
I_{\left(w_{1}, \ldots, w_{s}\right)}^{P}: \quad \omega_{P}\left(\sum_{j=1}^{s} w_{j}^{-1} h_{j}\right) \leq 0 .
$$

(c) For every standard maximal parabolic subgroup $P$ in $G$ and every choice of s-tuples $\left(w_{1}, \ldots, w_{s}\right) \in\left(W^{P}\right)^{s}$ such that

$$
\left[X_{w_{1}}^{P}\right] \cdots\left[X_{w_{s}}^{P}\right]=\left[X_{e}^{P}\right]
$$

the above inequality $I_{\left(w_{1}, \ldots, w_{s}\right)}^{P}$ holds.

(d) For every standard maximal parabolic subgroup $P$ in $G$ and every choice of s-tuples $\left(w_{1}, \ldots, w_{s}\right) \in\left(W^{P}\right)^{s}$ such that

$$
\left[X_{w_{1}}^{P}\right] \odot_{0} \cdots \odot_{0}\left[X_{w_{s}}^{P}\right]=\left[X_{e}^{P}\right]
$$

the above inequality $I_{\left(w_{1}, \ldots, w_{s}\right)}^{P}$ holds.

The equivalence of (a) and (b) in the above theorem for general $G$ is due to Knutson-Tao (2000). Kapovich-Leeb-Millson (2009) showed the equivalence of (a) and (c). The equivalence of (a) and (d) is due to Belkale-Kumar (2006). If we specialize the above Theorem for $G=\mathrm{SL}(n)$, then, in the view of Theorem 14, the equivalence of (a) and (b) is nothing but Horn's conjecture (Corollary 15) solved by combining the work of Klyachko (1998) with the work of Knutson-Tao (1999). (It may be remarked that the proofs of Horn's conjecture and Theorem 14 are recursively interdependent in the sense that the validity of Theorem 14 for $\mathrm{SL}(n)$ proves Horn's conjecture for $\mathfrak{s l}(n)$ (by using the equivalence of (a) and (b) in Theorem 2 for $G=\operatorname{SL}(n))$ but the proof of Theorem 14 for $\operatorname{SL}(n)$ requires the validity of Horn's conjecture for $\mathfrak{s l}(r)$, for $r<n$.) In this case, the equivalence of (a) and (c) is due to Belkale (2001). In this case, every maximal parabolic subgroup $P$ is cominuscule and hence the deformed product $\odot_{0}$ in $H^{*}(G / P)$ coincides with the standard cup product. Hence the parts (c) and (d) are the same in this case.

Because of the identification (3), the above theorem allows us to determine the saturated tensor semigroup $\Gamma_{s}(G)$ (see Theorems 12 and 24 for a precise statement).

The proof of the equivalence of the (a) and (b) parts of the above theorem follows from the Hilbert-Mumford criterion for semistability (cf. Proposition 8) and the determination of the Mumford index as in Lemma 9. The proof of the equivalence of the (a) and (d) parts is more delicate and relies on Kempf's maximally destabilizing one-parameter subgroups and Kempf's parabolic subgroups associated to unstable 


\section{SHRAWAN KUMAR}

points. In addition, the notion of Levi-movability plays a fundamental role in the proof.

As proved by Ressayre (2010), the inequalities given by the (d) part of the above theorem form an irredundant system of inequalities determining the cone $\bar{\Gamma}_{s}(\mathfrak{g})$ (cf. Corollary 36). (As mentioned above, for $\mathfrak{g}=\mathfrak{s l}(n)$ it was proved by Knutson-Tao-Wodward.) Ressayre's proof relies on the notion of well-covering pairs (cf. Definition 9), which is equivalent to the notion of Levi-movability with cup product 1 (cf. Lemma 32).

The eigencone $\bar{\Gamma}_{3}(\mathfrak{g})$ for the ranks 2 and 3 simple Lie algebras $\mathfrak{g}$ is explicitly determined in Section 14. For $\mathfrak{g}$ of rank 2, it is due to Kapovich-Leeb-Millson (2009) and for $\mathfrak{g}$ of rank 3, it is due to Kumar-Leeb-Millson (2003). The description relies on the above theorem (the equivalence of (a) and (d)). As shown by KumarLeeb-Millson (2003), the (c) part of the above theorem gives rise to 126 inequalities for $\mathfrak{g}$ of type $B_{3}$ or $C_{3}$, whereas by the (d) part one gets only 93 inequalities.

Let $\mathfrak{g}$ be a simple Lie algebra with a diagram automorphism $\sigma$ and let $\mathfrak{k}$ be the fixed subalgebra (which is necessarily simple again). Then, as shown by BelkaleKumar (2010) for the pairs $(s l(2 n), s p(2 n))$ and $(s l(2 n+1), s o(2 n+1))$, and by Braley (2012) and Lee (2012) for the other pairs, the eigencone $\bar{\Gamma}_{s}(\mathfrak{k})$ of $\mathfrak{k}$ is the intersection of the eigencone $\bar{\Gamma}_{s}(\mathfrak{g})$ of $\mathfrak{g}$ with the dominant chamber of $\mathfrak{k}$ (cf. Theorem $42)$. The proof for the pair $(s l(2 n), s p(2 n))$ (resp. $(s l(2 n+1), s o(2 n+1)))$ relies on the result that any collection of Schubert varieties in any Grassmannian can be moved by elements of $\mathrm{Sp}(2 n)$ (resp. $\mathrm{SO}(2 n+1)$ ) so that their intersection is proper (cf. Theorems 37 and 39). The proof in the other cases relies on the comparison between the intersection product of the partial flag varieties $G / P$ of $G$ (corresponding to the maximal parabolic subgroups $P$ of $G$ ) with that of the deformed product in the partial flag varieties $K / Q$ of $K$ (corresponding to the maximal parabolic subgroups $Q$ of $K$ ).

An 'explicit' determination of the eigencone $\bar{\Gamma}_{s}(\mathfrak{g})$ of $\mathfrak{g}$ via Theorem 2 hinges upon understanding the product $\odot_{0}$ in $H^{*}(G / P)$ in the Schubert basis, for all the maximal parabolic subgroups $P$. Clearly, the product $\odot_{0}$ is easier to understand than the usual cup product (which is the subject matter of Schubert Calculus) since in general 'many more' terms in the product $\odot_{0}$ in the Schubert basis drop out. However, the product $\odot_{0}$ has a drawback in that it is not functorial, in general even for the standard projections $\pi: G / P \rightarrow G / Q$ for parabolic subgroups $P \subset Q$. But, for certain embeddings of flag varieties $\iota: G / P \hookrightarrow \widehat{G} / \widehat{P}$, RessayreRichmond (2011) defined a certain 'deformed' pull-back map in cohomology which respects the product $\odot_{0}$ (cf. Theorem 58). A decomposition formula for the structure constants in $\odot_{0}$ is obtained by Richmond (2012) (also by Ressayre (2011)) (cf. Theorems 63, 64 and Corollary 65). We give the tables of the deformed product $\odot$ for the groups of type $B_{2}, G_{2}, B_{3}$, and $C_{3}$ and for any maximal parabolic subgroups in Section 13.

Also, as shown by Belkale-Kumar (2006), the deformed product $\odot_{0}$ in $H^{*}(G / P)$ is connected with the Lie algebra cohomology of the nil-radical $\mathfrak{u}_{P}$ of the parabolic subalgebra $\mathfrak{p}$ (cf. Theorem 56).

Let $G \subset \widehat{G}$ be connected reductive complex algebraic groups. Fix a maximal torus $H$ (resp. $\widehat{H})$ and a Borel subgroup $H \subset B($ resp. $\widehat{H} \subset \widehat{B})$ of $G($ resp. $\widehat{G})$ such 


\section{EIGENVALUE PROBLEM}

that $H \subset \widehat{H}$ and $B \subset \widehat{B}$. Define the saturated restriction semigroup

$$
\Gamma(G, \widehat{G})=\left\{(\lambda, \widehat{\lambda}) \in \Lambda_{+} \times \widehat{\Lambda}_{+}:[V(N \lambda) \otimes \widehat{V}(N \widehat{\lambda})]^{G} \neq 0 \text { for some } N \geq 1\right\} .
$$

In Section 7, Theorem 2 is generalized to the determination of $\Gamma(G, \widehat{G})$. Specifically, we have the following result due to Ressayre (2010) (cf. Theorems 33 and 35). (A weaker result was obtained by Knutson-Tao (2000).)

Any algebraic group morphism $\mathbb{G}_{m} \rightarrow H$ is called a one-parameter subgroup (for short, OPS) in $H$. Let $O(H)$ be the set of all the OPS in $H$.

Theorem 3. Assume that no nonzero ideal of $\mathfrak{g}$ is an ideal of $\widehat{\mathfrak{g}}$. Let $(\lambda, \widehat{\lambda}) \in$ $\Lambda_{+} \times \widehat{\Lambda}_{+}$. Then, the following three conditions are equivalent:

(a) $(\lambda, \widehat{\lambda}) \in \Gamma(G, \widehat{G})$.

(b) For any $G$-dominant $\delta \in O(H)$, and any $(w, \widehat{w}) \in W^{P(\delta)} \times \widehat{W}^{\widehat{P}(\delta)}$ such that $\left[X_{w}^{P(\delta)}\right] \cdot \iota^{*}\left(\left[\widehat{X}_{\widehat{w}}^{\widehat{P}(\delta)}\right]\right) \neq 0$ in $H^{*}(G / P(\delta), \mathbb{Z})$, where $\widehat{X}_{\widehat{w}}^{\widehat{P}}{ }^{(\delta)}:=\widehat{\widehat{B} \widehat{w} \widehat{P}(\delta) / \widehat{P}(\delta)} \subset$ $\widehat{G} / \widehat{P}(\delta)$ and $\iota: G / P(\delta) \rightarrow \widehat{G} / \widehat{P}(\delta)$ is the canonical embedding, we have

$$
I_{(w, \widehat{w})}^{\delta}: \quad \lambda(w \dot{\delta})+\widehat{\lambda}(\widehat{w} \dot{\delta}) \leq 0,
$$

where $P(\delta)$ (resp. $\widehat{P}(\delta))$ is the Kempf's parabolic in $G$ (resp. $\widehat{G}$ ) defined by the identity (11).

(c) For any $\operatorname{OPS} \delta_{i} \in \mathfrak{S}(G, \widehat{G})$ and any $(w, \widehat{w}) \in W^{P\left(\delta_{i}\right)} \times \widehat{W}^{\widehat{P}\left(\delta_{i}\right)}$ such that

$$
\begin{aligned}
& \left(c_{1}\right)\left[X_{w}^{P\left(\delta_{i}\right)}\right] \cdot \iota^{*}\left(\left[\widehat{X}_{\widehat{w}}^{\widehat{P}\left(\delta_{i}\right)}\right]\right)=\left[X_{e}^{P\left(\delta_{i}\right)}\right] \in H^{*}\left(G / P\left(\delta_{i}\right), \mathbb{Z}\right) \text {, and } \\
& \left(c_{2}\right) \gamma_{e}^{P\left(\delta_{i}\right)}\left(\dot{\delta}_{i}\right)-\gamma_{w}^{P\left(\delta_{i}\right)}\left(\dot{\delta}_{i}\right)=\widehat{\gamma}_{\widehat{w}}^{\widehat{P}\left(\delta_{i}\right)}\left(\dot{\delta}_{i}\right) \text {, }
\end{aligned}
$$

the above inequality $I_{(w, \widehat{w})}^{\delta_{i}}$ is satisfied, where the set $\mathfrak{S}(G, \widehat{G})$ is defined in Definition 10 and $\gamma_{w}^{P\left(\delta_{i}\right)}\left(\dot{\delta}_{i}\right)$ (resp. $\left.\widehat{\gamma}_{\widehat{w}}^{\widehat{w}\left(\delta_{i}\right)}\left(\dot{\delta}_{i}\right)\right)$ are given by the identities (45) (resp. (46)).

Moreover, the set of inequalities provided by the (c)-part is an irredundant system of inequalities describing the cone $\Gamma(G, \widehat{G})_{\mathbb{R}}$ inside $\Lambda_{+}(\mathbb{R}) \times \widehat{\Lambda}_{+}(\mathbb{R})$, where $\Lambda_{+}(\mathbb{R})$ denotes the cone inside $\Lambda \otimes_{\mathbb{Z}} \mathbb{R}$ generated by $\Lambda_{+}$and $\Gamma(G, \widehat{G})_{\mathbb{R}}$ is the cone generated by $\Gamma(G, \widehat{G})$.

Let $G$ be a connected semisimple group. The saturation problem aims at connecting the tensor product semigroup

$$
T_{s}(G):=\left\{\left(\lambda_{1}, \ldots, \lambda_{s}\right) \in \Lambda_{+}^{s}:\left[V\left(\lambda_{1}\right) \otimes \cdots \otimes V\left(\lambda_{s}\right)\right]^{G} \neq 0\right\}
$$

with the saturated tensor product semigroup $\Gamma_{s}(G)$. An integer $d \geq 1$ is called a saturation factor for $G$, if for any $(\lambda, \mu, \nu) \in \Gamma_{3}(G)$ such that $\lambda+\mu+\nu \in Q$, then $(d \lambda, d \mu, d \nu) \in T_{3}(G)$, where $Q$ is the root lattice of $G$. Such a $d$ exists by Corollary 44. If $d=1$ is a saturation factor for $G$, we say that the saturation property holds for $G$.

The saturation theorem of Knutson-Tao (1999) mentioned above, proved by using their 'honeycomb model,' asserts that the saturation property holds for $G=$ 


\section{SHRAWAN KUMAR}

$\mathrm{SL}(n)$. Other proofs of their result are given by Derksen-Weyman (2000), Belkale (2006) and Kapovich-Millson (2008).

The following general result (though not optimal) on saturation factor is obtained by Kapovich-Millson (2008) (cf. the Appendix).

Theorem 4. For any connected simple $G, d=k_{\mathfrak{g}}^{2}$ is a saturation factor, where $k_{\mathfrak{g}}$ is the least common multiple of the coefficients of the highest root $\theta$ of the Lie algebra $\mathfrak{g}$ of $G$ written in terms of the simple roots $\left\{\alpha_{1}, \ldots, \alpha_{\ell}\right\}$.

Kapovich-Millson (2006) made the very interesting conjecture that if $G$ is simply-laced, then the saturation property holds for $G$. Apart from $G=\operatorname{SL}(n)$, the only other simply-connected, simple, simply-laced group $G$ for which the above conjecture is known so far is $G=\operatorname{Spin}(8)$, proved by Kapovich-Kumar-Millson (2009) by explicit calculation using the equivalence of (a) and (d) in Theorem 2.

For the classical groups $\mathrm{SO}(n)(n \geq 5)$ and $\mathrm{Sp}(2 \ell)(\ell \geq 2), 2$ is a saturation factor. It was proved by Belkale-Kumar (2010) for the groups $\mathrm{SO}(2 \ell+1)$ and $\mathrm{Sp}(2 \ell)$ by using geometric techniques. Sam (2012) proved it for $\mathrm{SO}(2 \ell)$ (and also for $\mathrm{SO}(2 \ell+1)$ and $\mathrm{Sp}(2 \ell)$ ) via the quiver approach (following the proof by DerksenWeyman (2010) for $G=\mathrm{SL}(n)$ ). (Observe that the general result of KapovichMillson gives a saturation factor of 4 in these cases.)

We recall, in Section 10, a 'rigidity' result for the SL $(n)$-representations due to Knutson-Tao-Woodward (2004), which was conjectured by Fulton and also its generalization to an arbitrary reductive group by Belkale-Kumar-Ressayre (2012) (cf. Theorems 53 and 54).

We refer the reader to the survey article of Fulton $\left[\mathrm{F}_{2}\right]$ on the Hermitian eigenvalue problem; and the Bourbaki talk by Brion $[\mathrm{Br}]$.

Acknowledgements. It is my pleasure to thank Prakash Belkale who introduced me to the eigenvalue problem for my joint works with him. I thank N. Ressayre for going through the article and for his comments. I also thank M. Kapovich, B. Leeb, J. Millson, and N. Ressayre for my joint works with them on the subject. We thank the referee for the comments. I gratefully acknowledge the support from the NSF grant DMS-1201310.

\section{Notation}

Let $G$ be a semisimple connected complex algebraic group. We choose a Borel subgroup $B$ and a maximal torus $H \subset B$ and let $W=W_{G}:=N_{G}(H) / H$ be the associated Weyl group, where $N_{G}(H)$ is the normalizer of $H$ in $G$. Let $P \supseteq B$ be a (standard) parabolic subgroup of $G$ and let $U=U_{P}$ be its unipotent radical. Consider the Levi subgroup $L=L_{P}$ of $P$ containing $H$, so that $P$ is the semidirect product of $U$ and $L$. Then, $B_{L}:=B \cap L$ is a Borel subgroup of $L$. Let $\Lambda=\Lambda(H)$ denote the character group of $H$, i.e., the group of all the algebraic group morphisms $H \rightarrow \mathbb{G}_{m}$. Clearly, $W$ acts on $\Lambda$. We denote the Lie algebras of $G, B, H, P, U, L, B_{L}$ by the corresponding Gothic characters: $\mathfrak{g}, \mathfrak{b}, \mathfrak{h}, \mathfrak{p}, \mathfrak{u}, \mathfrak{l}, \mathfrak{b} \mathfrak{b}_{L}$ respectively. We will often identify an element $\lambda$ of $\Lambda$ (via its derivative $\dot{\lambda}$ ) by an element of $\mathfrak{h}^{*}$. Let $R=R_{\mathfrak{g}} \subset \mathfrak{h}^{*}$ be the set of roots of $\mathfrak{g}$ with respect to the Cartan subalgebra $\mathfrak{h}$ and let $R^{+}$be the set of positive roots (i.e., the set of roots of $\mathfrak{b}$ ). 
Similarly, let $R_{\mathfrak{l}}$ be the set of roots of $\mathfrak{l}$ with respect to $\mathfrak{h}$ and $R_{\mathfrak{l}}^{+}$be the set of roots of $\mathfrak{b}_{L}$. Let $\Delta=\left\{\alpha_{1}, \ldots, \alpha_{\ell}\right\} \subset R^{+}$be the set of simple roots, $\left\{\alpha_{1}^{\vee}, \ldots, \alpha_{\ell}^{\vee}\right\} \subset \mathfrak{h}$ the corresponding simple coroots and $\left\{s_{1}, \ldots, s_{\ell}\right\} \subset W$ the corresponding simple reflections, where $\ell$ is the rank of $G$. We denote by $\Delta(P)$ the set of simple roots contained in $R_{\mathfrak{l}}$. For any $1 \leq j \leq \ell$, define the element $x_{j} \in \mathfrak{h}$ by

$$
\alpha_{i}\left(x_{j}\right)=\delta_{i, j}, \forall 1 \leq i \leq \ell .
$$

Recall that if $W_{P}$ is the Weyl group of $P$ (which is, by definition, the Weyl Group $W_{L}$ of $L$ ), then in each coset of $W / W_{P}$ we have a unique member $w$ of minimal length. This satisfies (cf. [ $\mathrm{K}_{1}$, Exercise 1.3.E]):

$$
w B_{L} w^{-1} \subseteq B .
$$

Let $W^{P}$ be the set of the minimal length representatives in the cosets of $W / W_{P}$.

For any $w \in W^{P}$, define the Schubert cell:

$$
C_{w}^{P}:=B w P / P \subset G / P .
$$

Then, it is a locally closed subvariety of $G / P$ isomorphic with the affine space $\mathbb{A}^{\ell(w)}, \ell(w)$ being the length of $w$ (cf. [J, Part II, Chap. 13]). Its closure is denoted by $X_{w}^{P}$, which is an irreducible (projective) subvariety of $G / P$ of dimension $\ell(w)$. We denote the point $w P \in C_{w}^{P}$ by $\dot{w}$. We abbreviate $X_{w}^{B}$ by $X_{w}$.

Let $\mu\left(X_{w}^{P}\right)$ denote the fundamental class of $X_{w}^{P}$ considered as an element of the singular homology with integral coefficients $H_{2 \ell(w)}(G / P, \mathbb{Z})$ of $G / P$. Then, from the Bruhat decomposition, the elements $\left\{\mu\left(X_{w}^{P}\right)\right\}_{w \in W^{P}}$ form a $\mathbb{Z}$-basis of $H_{*}(G / P, \mathbb{Z})$. Let $\left\{\left[X_{w}^{P}\right]\right\}_{w \in W^{P}}$ be the Poincaré dual basis of the singular cohomology with integral coefficients $H^{*}(G / P, \mathbb{Z})$. Thus, $\left[X_{w}^{P}\right] \in H^{2(\operatorname{dim} G / P-\ell(w))}(G / P, \mathbb{Z})$. Similarly, let $\left\{\epsilon_{w}^{P}\right\}_{w \in W^{P}}$ be the basis of $H^{*}(G / P, \mathbb{Z})$ dual to the basis $\left\{\mu\left(X_{w}^{P}\right)\right\}_{w \in W^{P}}$ of $H_{*}(G / P, \mathbb{Z})$ under the standard pairing, i.e., for any $v, w \in W^{P}$, we have

$$
\epsilon_{v}^{P}\left(\mu\left(X_{w}^{P}\right)\right)=\delta_{v, w} .
$$

Then, for any $w \in W^{P}$, by [KuLM, Prop. 2.6],

$$
\epsilon_{w}^{P}=\left[X_{w_{o} w w_{o}^{P}}^{P}\right],
$$

where $w_{o}$ (resp. $w_{o}^{P}$ ) is the longest element of the Weyl group $W\left(\right.$ resp. $W_{P}$ ). (Observe that $w_{o} w w_{o}^{P} \in W^{P}$ for any $w \in W^{P}$.)

An element $\lambda \in \Lambda$ is called dominant (resp. dominant regular) if $\dot{\lambda}\left(\alpha_{i}^{\vee}\right) \geq 0$ (resp. $\dot{\lambda}\left(\alpha_{i}^{\vee}\right)>0$ ) for all the simple coroots $\alpha_{i}^{\vee}$. Let $\Lambda_{+}\left(\right.$resp. $\left.\Lambda_{++}\right)$denote the set of all the dominant (resp. dominant regular) characters. The set of isomorphism classes of irreducible (finite-dimensional) representations of $G$ is parameterized by $\Lambda_{+}$via the highest weights of irreducible representations. For $\lambda \in \Lambda_{+}$, we denote by $V(\lambda)$ the corresponding irreducible representation (of highest weight $\lambda$ ). The dual representation $V(\lambda)^{*}$ is isomorphic with $V\left(\lambda^{*}\right)$, where $\lambda^{*}$ is the weight $-w_{o} \lambda$. 


\section{SHRAWAN KUMAR}

The $\mu$-weight space of $V(\lambda)$ is denoted by $V(\lambda)_{\mu}$. For $\lambda \in \Lambda_{+}$, let $P(\lambda)$ be the set of weights of $V(\lambda)$. We denote the fundamental weights by $\left\{\omega_{i}\right\}_{1 \leq i \leq \ell}$, i.e.,

$$
\omega_{i}\left(\alpha_{j}^{\vee}\right)=\delta_{i, j} .
$$

For any $\lambda \in \Lambda$, we have a $G$-equivariant line bundle $\mathcal{L}(\lambda)$ on $G / B$ associated to the principal $B$-bundle $G \rightarrow G / B$ via the one-dimensional $B$-module $\lambda^{-1}$. (Any $\lambda \in \Lambda$ extends uniquely to a character of $B$.) The one-dimensional $B$-module $\lambda$ is also denoted by $\mathbb{C}_{\lambda}$.

All the schemes are considered over the base field of complex numbers $\mathbb{C}$. The varieties are reduced (but not necessarily irreducible) schemes.

\section{Determination of the eigencone/saturated tensor cone}

Let the notation and assumptions be as in Section 2. In particular, $G$ is a connected semisimple complex algebraic group. Fix a positive integer $s$ and define the saturated tensor semigroup

$\Gamma_{s}(G)=\left\{\left(\lambda_{1}, \ldots, \lambda_{s}\right) \in \Lambda_{+}^{s}:\left[V\left(N \lambda_{1}\right) \otimes \cdots \otimes V\left(N \lambda_{s}\right)\right]^{G} \neq 0\right.$ for some $\left.N \geq 1\right\}$.

Let

$$
\mathfrak{h}_{+}:=\left\{x \in \mathfrak{h}: \alpha_{i}(x) \in \mathbb{R}_{+} \text {for all the simple roots } \alpha_{i}\right\}
$$

be the dominant chamber in $\mathfrak{h}$, where $\mathbb{R}_{+}$is the set of nonnegative real numbers. Define the eigencone

$\bar{\Gamma}_{s}(\mathfrak{g})=\left\{\left(h_{1}, \ldots, h_{s}\right) \in \mathfrak{h}_{+}^{s}:\right.$ there exist $k_{1}, \ldots, k_{s} \in K$ with $\left.\sum_{j=1}^{s}\left(\operatorname{Ad} k_{j}\right) h_{j}=0\right\}$,

where $K \subset G$ is a fixed maximal compact subgroup. Then, $\bar{\Gamma}_{s}(\mathfrak{g})$ depends only upon the Lie algebra $\mathfrak{g}$ and the choices of its Borel subalgebra $\mathfrak{b}$ and the Cartan subalgebra $\mathfrak{h}$.

Under the identification $\varphi: \mathfrak{h} \stackrel{\sim}{\rightarrow} \mathfrak{h}^{*}$ (via the Killing form) $\Gamma_{s}(G)$ corresponds to the set of integral points of $\bar{\Gamma}_{s}(\mathfrak{g})$. Specifically, we have the following result essentially following from Mumford [N, Appendix] (also see $[\mathrm{Sj}$, Thm. 7.6] and [Br, Thm. 1.3]).

\section{Theorem 5.}

$$
\varphi\left(\bar{\Gamma}_{s}(\mathfrak{g})\right) \cap \Lambda_{+}^{s}=\Gamma_{s}(G) .
$$

Proof. For $h=\left(h_{1}, \ldots, h_{s}\right) \in \mathfrak{h}_{+}^{s}$, let

$$
\mathcal{O}_{h}:=\left(K \cdot h_{1}\right) \times \cdots \times\left(K \cdot h_{s}\right) \subset(i \mathfrak{k})^{s} .
$$

Then, $K$ acts on $\mathcal{O}_{h}$ diagonally. Let $m_{h}: \mathcal{O}_{h} \rightarrow i \mathfrak{k}^{*} \simeq i \mathfrak{k}$ be the corresponding moment map, where the last identification is via the Killing form. Then, it is easy to see that $m_{h}\left(y_{1}, \ldots, y_{s}\right)=y_{1}+\cdots+y_{s}$, for $y_{j} \in K \cdot h_{j}$. Hence,

$$
h \in \bar{\Gamma}_{s}(\mathfrak{g}) \Leftrightarrow 0 \in \operatorname{Im}\left(m_{h}\right) .
$$




\section{EIGENVALUE PROBLEM}

Now, take $h=\left(h_{1}, \ldots, h_{s}\right) \in \mathfrak{h}_{+}^{s}$ such that $\lambda=\varphi(h)=\left(\lambda_{1}, \ldots, \lambda_{s}\right) \in \Lambda_{+}^{s}$, where $\lambda_{j}:=\varphi\left(h_{j}\right)$. Consider the closed subvariety

$$
X_{\lambda}:=G \cdot\left[v_{\lambda_{1}}\right] \times \cdots \times G \cdot\left[v_{\lambda_{s}}\right] \subset \mathbb{P}\left(V\left(\lambda_{1}\right)\right) \times \cdots \times \mathbb{P}\left(V\left(\lambda_{s}\right)\right),
$$

where $\left[v_{\lambda_{j}}\right]$ is the line through the highest weight vector in $V\left(\lambda_{j}\right)$.

It is easy to see that $K \cdot h_{j}$ is diffeomorphic with $G \cdot\left[v_{\lambda_{j}}\right]$ as symplectic $K$ manifolds. In particular, there exists a $K$-equivariant symplectic diffeomorphism $\theta: \mathcal{O}_{h} \rightarrow X_{\lambda}$ (under the diagonal action of $K$ ). Hence the following diagram is commutative:

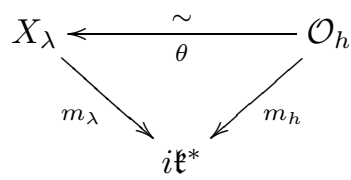

where $m_{\lambda}: X_{\lambda} \rightarrow i \mathfrak{k}^{*}$ is the moment map for the $K$-variety $X_{\lambda}$. Let $\overline{\mathbb{L}}(\lambda)$ be the ample line bundle on $X_{\lambda}$ which is the restriction of the line bundle $\mathcal{O}(1) \otimes \cdots \otimes \mathcal{O}(1)$ on $\mathbb{P}\left(V\left(\lambda_{1}\right)\right) \times \cdots \times \mathbb{P}\left(V\left(\lambda_{s}\right)\right)$. Now, $X_{\lambda}$ has a $G$-semistable point with respect to the ample line bundle $\overline{\mathbb{L}}(\lambda)$ if and only if $0 \in \operatorname{Im}\left(m_{\lambda}\right)$ (cf. [MFK, Thm. 8.3]). Further, by the definition, $X_{\lambda}$ has a $G$-semistable point with respect to the line bundle $\overline{\mathbb{L}}(\lambda)$ if and only if $H^{0}\left(X_{\lambda}, \overline{\mathbb{L}}(\lambda)^{\otimes N}\right)^{G} \neq 0$ for some $N>0$. The latter of course is equivalent (by the Borel-Weil theorem) to

$\left[V\left(N \lambda_{1}\right)^{*} \otimes \cdots \otimes V\left(N \lambda_{s}\right)^{*}\right]^{G} \neq 0 \Leftrightarrow\left[V\left(N \lambda_{1}\right) \otimes \cdots \otimes V\left(N \lambda_{s}\right)\right]^{G} \neq 0 \Leftrightarrow \lambda \in \Gamma_{s}(G)$.

This, together with (10), proves the theorem.

We recall the following transversality Kleiman's theorem (see [BK ${ }_{1}$, Prop. 3]).

Theorem 6. Let a connected algebraic group $S$ act transitively on a smooth variety $X$ and let $X_{1}, \ldots, X_{s}$ be irreducible locally closed subvarieties of $X$. Then, there exists a nonempty open subset $V \subseteq S^{s}$ such that for $\left(g_{1}, \ldots, g_{s}\right) \in V$, the intersection $\bigcap_{j=1}^{s} g_{j} X_{j}$ is proper (possibly empty) and dense in $\bigcap_{j=1}^{s} g_{j} \bar{X}_{j}$.

Moreover, if $X_{j}$ are smooth varieties, we can find such a $V$ with the additional property that for $\left(g_{1}, \ldots, g_{s}\right) \in V, \bigcap_{j=1}^{s} g_{j} X_{j}$ is transverse at each point of intersection.

The following result follows from [ $\mathrm{F}_{1}$, Prop. 7.1 and Sect. 12.2].

Proposition 7. Let $w_{1}, \ldots, w_{s} \in W^{P}$ and let $g_{1}, \ldots, g_{s} \in G$ be such that the intersection $Y:=g_{1} X_{w_{1}}^{P} \cap \cdots \cap g_{s} X_{w_{s}}^{P}$ is proper (or empty) inside $G / P$. Then, we have

$$
\left[X_{w_{1}}^{P}\right] \ldots\left[X_{w_{s}}^{P}\right]=[Y]
$$

where $[Y]$ denotes the Poincaré dual of the fundamental class of the pure (but not necessarily irreducible) scheme $Y$.

Moreover, for any irreducible component $C$ of $Y$, writing

$$
[C]=\sum_{w \in W^{P}} n_{w}\left[X_{w}^{P}\right]
$$

for some (unique) $n_{w} \in \mathbb{Z}$, we have $n_{w} \in \mathbb{Z}_{+}$. 


\section{SHRAWAN KUMAR}

A Review of Geometric Invariant Theory. We will need to consider in Sections 5 and 6 the Geometric Invariant Theory (GIT) in a nontraditional setting, where a nonreductive group acts on a nonprojective variety. First we recall the following definition due to Mumford.

Definition 1. Let $S$ be any (not necessarily reductive) algebraic group acting on a (not necessarily projective) variety $\mathbb{X}$ and let $\mathbb{L}$ be an $S$-equivariant line bundle on $\mathbb{X}$. Any algebraic group morphism $\mathbb{G}_{m} \rightarrow S$ is called a one-parameter subgroup (for short, OPS) in $S$. Let $O(S)$ be the set of all the OPS in $S$. Take any $x \in \mathbb{X}$ and $\delta \in O(S)$ such that the $\operatorname{limit}_{\lim _{t \rightarrow 0}} \delta(t) x$ exists in $\mathbb{X}$ (i.e., the morphism $\delta_{x}: \mathbb{G}_{m} \rightarrow X$ given by $t \mapsto \delta(t) x$ extends to a morphism $\left.\widetilde{\delta}_{x}: \mathbb{A}^{1} \rightarrow X\right)$. Then, following Mumford, define a number $\mu^{\mathbb{L}}(x, \delta)$ as follows: Let $x_{o} \in X$ be the point $\widetilde{\delta}_{x}(0)$. Since $x_{o}$ is $\mathbb{G}_{m}$-invariant via $\delta$, the fiber of $\mathbb{L}$ over $x_{o}$ is a $\mathbb{G}_{m}$-module; in particular, it is given by a character of $\mathbb{G}_{m}$. This integer is defined as $\mu^{\mathbb{L}}(x, \delta)$.

We record the following standard properties of $\mu^{\mathbb{L}}(x, \delta)$; see [MFK, Chap. $\left.2, \S 1\right]$ :

Proposition 8. For any $x \in \mathbb{X}$ and $\delta \in O(S)$ such that $\lim _{t \rightarrow 0} \delta(t) x$ exists in $\mathbb{X}$, we have the following (for any $S$-equivariant line bundles $\mathbb{L}, \mathbb{L}_{1}, \mathbb{L}_{2}$ ):

(a) $\mu^{\mathbb{L}_{1} \otimes \mathbb{L}_{2}}(x, \delta)=\mu^{\mathbb{L}_{1}}(x, \delta)+\mu^{\mathbb{L}_{2}}(x, \delta)$.

(b) If there exists $\sigma \in H^{0}(\mathbb{X}, \mathbb{L})^{S}$ such that $\sigma(x) \neq 0$, then $\mu^{\mathbb{L}}(x, \delta) \geq 0$.

(c) If $\mu^{\mathbb{L}}(x, \delta)=0$, then any element of $H^{0}(\mathbb{X}, \mathbb{L})^{S}$ which does not vanish at $x$ does not vanish at $\lim _{t \rightarrow 0} \delta(t) x$ as well.

(d) For any $S$-variety $\mathbb{X}^{\prime}$ together with an $S$-equivariant morphism $f: \mathbb{X}^{\prime} \rightarrow \mathbb{X}$ and any $x^{\prime} \in \mathbb{X}^{\prime}$ such that $\lim _{t \rightarrow 0} \delta(t) x^{\prime}$ exists in $\mathbb{X}^{\prime}$, we have $\mu^{f^{*}} \mathbb{L}\left(x^{\prime}, \delta\right)=$ $\mu^{\mathbb{L}}\left(f\left(x^{\prime}\right), \delta\right)$.

(e) (Hilbert-Mumford criterion) Assume that $\mathbb{X}$ is projective, $S$ is connected and reductive and $\mathbb{L}$ is ample. Then, $x \in \mathbb{X}$ is semistable (with respect to $\mathbb{L})$ if and only if $\mu^{\mathbb{L}}(x, \delta) \geq 0$, for all $\delta \in O(S)$.

In particular, if $x \in \mathbb{X}$ is semistable and $\delta$-fixed, then $\mu^{\mathbb{L}}(x, \delta)=0$.

Let $S$ be a connected reductive group. For an OPS $\delta \in O(S)$, define the associated Kempf's parabolic subgroup $P(\delta)$ of $S$ by

$$
P(\delta):=\left\{g \in S: \lim _{t \rightarrow 0} \delta(t) g \delta(t)^{-1} \text { exists in } S\right\}
$$

For an OPS $\delta \in O(S)$, let $\dot{\delta} \in \mathfrak{s}$ be its derivative at 1 .

Let $P$ be any standard parabolic subgroup of $G$ acting on $P / B_{L}$ via the left multiplication. We call $\delta \in O(P) P$-admissible if, for all $x \in P / B_{L}, \lim _{t \rightarrow 0} \delta(t) \cdot x$ exists in $P / B_{L}$. If $P=G$, then $P / B_{L}=G / B$ and any $\delta \in O(G)$ is $G$-admissible since $G / B$ is a projective variety.

Observe that, $B_{L}$ being the semidirect product of its commutator $\left[B_{L}, B_{L}\right]$ and $H$, any $\lambda \in \Lambda$ extends uniquely to a character of $B_{L}$. Thus, for any $\lambda \in \Lambda$, we have a $P$-equivariant line bundle $\mathcal{L}_{P}(\lambda)$ on $P / B_{L}$ associated to the principal $B_{L}$-bundle $P \rightarrow P / B_{L}$ via the one-dimensional $B_{L}$-module $\lambda^{-1}$. Thus, $\mathcal{L}_{G}(\lambda)=\mathcal{L}(\lambda)$, as defined in Section 2. We have taken the following lemma from [BK $\mathrm{BK}_{1}$, Lemma 14]. It is a generalization of the corresponding result in [BS, Sect. 4.2]. 
Lemma 9. Let $\delta \in O(H)$ be such that $\dot{\delta} \in \mathfrak{h}_{+}$. Then, $\delta$ is P-admissible and, moreover, for any $\lambda \in \Lambda$ and $x=u l B_{L} \in P / B_{L}\left(\right.$ for $\left.u \in U_{P}, l \in L_{P}\right)$, we have the following formula:

$$
\mu^{\mathcal{L}_{P}(\lambda)}(x, \delta)=-\lambda(w \dot{\delta})
$$

where $P_{L}(\delta):=P(\delta) \cap L$ and $w \in W_{P} / W_{P_{L}(\delta)}$ is any coset representative such that $l^{-1} \in B_{L} w P_{L}(\delta)$.

Proof. We first show that $\delta$ is $P$-admissible. Take $x=u l B_{L} \in P / B_{L}$, for $u \in U_{P}$ and $l \in L_{P}$. Then, $\delta(t) x=\delta(t) u \delta(t)^{-1}\left(\delta(t) l B_{L}\right)$. Now, since $\dot{\delta} \in \mathfrak{h}_{+}$and $u \in U_{P}$, it is easy to see that $\lim _{t \rightarrow 0} \delta(t) u \delta(t)^{-1}$ exists in $U_{P}$. Also, $\lim _{t \rightarrow 0} \delta(t) l B_{L}$ exists in $L / B_{L}$ since $L / B_{L}$ is a projective variety. Thus, $\delta$ is $P$-admissible.

We next calculate $\mu^{\mathcal{L}_{P}(\lambda)}(x, \delta)$ for $x=u l B_{L} \in P / B_{L}$. Write $l^{-1}=b_{l} \dot{w} q$, for some $b_{l} \in B_{L}$ and $q \in P_{L}(\delta) \supset B_{L}$ (where $\dot{w}$ is a representative of $w$ in the normalizer $N_{L}(H)$ of $H$ in $\left.L\right)$. Consider the OPS $b: \mathbb{G}_{m} \rightarrow B_{L}$ defined by $b(t)=b_{l} \dot{w} \delta(t)^{-1} \dot{w}^{-1} b_{l}^{-1}$. Then,

$$
\delta(t) u l b(t)=\delta(t) u q^{-1} \delta(t)^{-1} \dot{w}^{-1} b_{l}^{-1}
$$

In particular, by the definition of $P(\delta), \lim _{t \rightarrow 0} \delta(t) u l b(t)$ exists in $P$. Consider the $\mathbb{G}_{m}$-invariant section $\sigma(t)=[\delta(t) u l, 1]:=(\delta(t) u l, 1) \bmod B_{L}$ of $\delta_{x}^{*}\left(\mathcal{L}_{P}(\lambda)\right)$ over $\mathbb{G}_{m}$, where $\delta_{x}: \mathbb{G}_{m} \rightarrow P / B_{L}$ is the map $t \mapsto \delta(t) x$. Then, the section $\sigma(t)$ corresponds to the function $\mathbb{G}_{m} \rightarrow \mathbb{A}^{1}, t \mapsto \lambda^{-1}\left(b(t)^{-1}\right)$. From this we see that $\mu^{\mathcal{L}_{P}(\lambda)}(x, \delta)=-\lambda(w \dot{\delta})$.

Let $\lambda=\left(\lambda_{1}, \ldots, \lambda_{s}\right) \in \Lambda_{+}^{s}$ and let $\mathbb{L}(\lambda)$ denote the $G$-linearized line bundle $\mathcal{L}\left(\lambda_{1}\right) \otimes \cdots \otimes \mathcal{L}\left(\lambda_{s}\right)$ on $(G / B)^{s}$ (under the diagonal action of $G$ ). Then, there exist unique standard parabolic subgroups $P_{1}, \ldots, P_{s}$ such that the line bundle $\mathbb{L}(\lambda)$ descends as an ample line bundle $\overline{\mathbb{L}}(\lambda)$ on $\mathbb{X}(\lambda):=G / P_{1} \times \cdots \times G / P_{s}$. We call a point $x \in(G / B)^{s} G$-semistable (with respect to, not necessarily ample, $\mathbb{L}(\lambda)$ ) if its image in $\mathbb{X}(\lambda)$ under the canonical map $\pi:(G / B)^{s} \rightarrow \mathbb{X}(\lambda)$ is semistable with respect to the ample line bundle $\overline{\mathbb{L}}(\lambda)$. Now, one has the following celebrated theorem due to Klyachko [Kly] for $G=\mathrm{SL}(n)$, extended to general $G$ by KnutsonTao [BS].

Theorem 10. Let $\lambda_{1}, \ldots, \lambda_{s} \in \Lambda_{+}$. Then, the following are equivalent:

(a) $\left(\lambda_{1}, \ldots, \lambda_{s}\right) \in \Gamma_{s}(G)$.

(b) For every standard maximal parabolic subgroup $P$ and all Weyl group elements $w_{1}, \ldots, w_{s} \in W^{P} \simeq W / W_{P}$ such that

$$
\left[X_{w_{1}}^{P}\right] \ldots\left[X_{w_{s}}^{P}\right]=d\left[X_{e}^{P}\right] \text { for some } d \neq 0,
$$

the following inequality is satisfied:

$$
I_{\left(w_{1}, \ldots, w_{s}\right)}^{P}: \quad \sum_{j=1}^{s} \lambda_{j}\left(w_{j} x_{P}\right) \leq 0
$$

where $\alpha_{i_{P}}$ is the unique simple root not in the Levi of $P$ and $x_{P}:=x_{i_{P}}$. 


\section{SHRAWAN KUMAR}

Proof. Define the set $Y_{s} \subset G^{s}$ consisting of those $\left(g_{1}, \ldots, g_{s}\right) \in G^{s}$ such that $g_{1} X_{w_{1}}^{Q} \cap \ldots \cap g_{s} X_{w_{s}}^{Q}$ and $\left(g_{1} B w_{1} Q / Q\right) \cap \ldots \cap\left(g_{s} B w_{s} Q / Q\right)$ are proper intersections and such that the latter intersection is dense in $g_{1} X_{w_{1}}^{Q} \cap \ldots \cap g_{s} X_{w_{s}}^{Q}$ for all the standard parabolic subgroups $Q$ and all $w_{1}, \ldots, w_{s} \in W$. Then, by Theorem $6, Y_{s}$ contains a nonempty open subset of $G^{s}$.

Now, $\lambda=\left(\lambda_{1}, \ldots \lambda_{s}\right) \in \Lambda_{+}^{s}$ belongs to $\Gamma_{s}(G)$

$\Longleftrightarrow X^{s}=(G / B)^{s}$ contains a $G$-semistable point $y$ with respect to the line bundle $\mathbb{L}(\lambda)$ on $X^{s}$.

$\Longleftrightarrow \mu^{\mathbb{L}(\lambda)}(y, \sigma) \geq 0$ for all one-parameter subgroups $\sigma$ in $G$.

(To prove the first equivalence, observe that, for any $N \geq 0$,

$$
H^{0}\left(X^{s}, \mathbb{L}(N \lambda)\right) \simeq H^{0}(\mathbb{X}(\lambda), \overline{\mathbb{L}}(N \lambda))
$$

under the pull-back map. The second equivalence of course follows by the HilbertMumford criterion Proposition 8(e) together with 8(d).)

Proof of $(\mathrm{a}) \Rightarrow(\mathrm{b})$ in Theorem 10 :

Take $\lambda=\left(\lambda_{1}, \ldots, \lambda_{s}\right) \in \Gamma_{s}(G)$. Then, $X^{s}$ has a $G$-semistable point for the line bundle $\mathbb{L}(\lambda)$. Moreover, since the set of semistable points is open, we can take a semistable point $y=\left(g_{1} B, \ldots, g_{s} B\right)$ with $\left(g_{1}, \ldots, g_{s}\right) \in Y_{s}$.

Now, take a maximal parabolic $P$ and $w_{1}, \ldots, w_{s} \in W^{P}$ satisfying (12). Thus,

$$
\left(g_{1} B w_{1} P / P\right) \cap \ldots \cap\left(g_{s} B w_{s} P / P\right) \neq \varnothing .
$$

Take $g P \in\left(g_{1} B w_{1} P / P\right) \cap \ldots \cap\left(g_{s} B w_{s} P / P\right)$. Take the one-parameter subgroup of $G: \sigma=\operatorname{Exp}\left(t x_{P}\right)$.

Then, by Lemma 9 and Proposition 8,

$$
\mu^{\mathbb{L}(\lambda)}\left(g^{-1} y, \sigma\right)=\sum-\lambda_{j}\left(w_{j} x_{P}\right) \geq 0
$$

where the last inequality is by the Hilbert-Mumford criterion Proposition 8(e). This proves $(\mathrm{a}) \Rightarrow(\mathrm{b})$.

Proof of $(\mathrm{b}) \Rightarrow(\mathrm{a})$ : If (a) were false, then $\lambda=\left(\lambda_{1}, \ldots, \lambda_{s}\right) \notin \Gamma_{s}(G)$, i.e., $X^{s}$ has no $G$-semistable points for $\mathbb{L}(\lambda)$. Take any $\left(g_{1}, \ldots, g_{s}\right) \in Y_{s}$ and consider the point $y=\left(g_{1} B, \ldots, g_{s} B\right) \in X^{s}$. Since it is not a semistable point, there exists a one-parameter subgroup $\sigma=g^{-1} \operatorname{Exp}(t x) g$, for $x \in \mathfrak{h}_{+}$and $g \in G$ such that

$$
\mu^{\mathbb{L}(\lambda)}(y, \sigma)<0 \Leftrightarrow \mu^{\mathbb{L}(\lambda)}(g y, \operatorname{Exp}(t x))<0 .
$$

Let $Q$ be the Kempf's parabolic attached to $\operatorname{Exp}(t x)$. Then, by definition, $Q \supset B$ and the simple roots of the Levi of $Q$ are precisely those $\alpha_{i}$ such that $\alpha_{i}(x)=0$. Take $w_{1}, \ldots, w_{s} \in W^{Q}$ such that

$$
\left(g g_{j}\right)^{-1} \in B w_{j} Q \quad \forall 1 \leq j \leq s .
$$


Thus, by Lemma 9,

$$
\mu^{\mathbb{L}(\lambda)}(g y, \operatorname{Exp} t x)=-\sum_{j=1}^{s} \lambda_{j}\left(w_{j} x\right)<0
$$

In particular, there exists a maximal parabolic $P \supset Q$ such that

$$
\sum_{j=1}^{s} \lambda_{j}\left(w_{j} x_{P}\right)>0
$$

Now, by (13),

$$
g g_{1} X_{w_{1}}^{Q} \cap \ldots \cap g g_{s} X_{w_{s}}^{Q} \quad \text { is nonempty. }
$$

In particular, $g g_{1} X_{w_{1}}^{P} \cap \ldots \cap g g_{s} X_{w_{s}}^{P}$ is nonempty and since $\left(g g_{1}, \ldots, g g_{s}\right) \in Y_{s}$, $g g_{1} X_{w_{1}}^{P} \cap \ldots \cap g g_{s} X_{w_{s}}^{P}$ is a proper intersection. Thus, by Proposition 7 , the cup product

$$
\left[X_{w_{1}}^{P}\right] \ldots\left[X_{w_{s}}^{P}\right] \neq 0
$$

Hence, there exists a $w_{s}^{\prime} \leq w_{s}$ such that $w_{s}^{\prime} \in W^{P}$ and

$$
\left[X_{w_{1}}^{P}\right] \ldots\left[X_{w_{s-1}}^{P}\right] \cdot\left[X_{w_{s}^{\prime}}^{P}\right]=d\left[X_{e}^{P}\right] \text {, for some } d \neq 0 \text {. }
$$

Now, by the inequality $I_{\left(w_{1}, \ldots, w_{s-1}, w_{s}^{\prime}\right)}^{P}$ in (b), we get that

$$
\left(\sum_{j=1}^{s-1} \lambda_{j}\left(w_{j} x_{P}\right)\right)+\lambda_{s}\left(w_{s}^{\prime} x_{P}\right) \leq 0
$$

But since $w_{s}^{\prime} \leq w_{s}$, we have

$$
\lambda_{s}\left(w_{s}^{\prime} x_{P}\right) \geq \lambda_{s}\left(w_{s} x_{P}\right)
$$

by, e.g., [K $\mathrm{K}_{1}$, Lemma 8.3.3]. Thus, we get

$$
\sum_{j=1}^{s} \lambda_{j}\left(w_{j} x_{P}\right) \leq\left(\sum_{j=1}^{s-1} \lambda_{j}\left(w_{j} x_{P}\right)\right)+\lambda_{s}\left(w_{s}^{\prime} x_{P}\right) \leq 0 .
$$

This contradicts (14) and hence proves that (a) is true.

The following result follows easily by combining Theorems 10 and 5 . For a maximal parabolic $P$, let $\alpha_{i_{P}}$ be the unique simple root not in the Levi of $P$. Then, we set $\omega_{P}:=\omega_{i_{P}}$. 


\section{SHRAWAN KUMAR}

Corollary 11. Let $\left(h_{1}, \ldots, h_{s}\right) \in \mathfrak{h}_{+}^{s}$. Then, the following are equivalent:

(a) $\left(h_{1}, \ldots, h_{s}\right) \in \bar{\Gamma}_{s}(\mathfrak{g})$.

(b) For every standard maximal parabolic subgroup $P$ in $G$ and every choice of s-tuples $\left(w_{1}, \ldots, w_{s}\right) \in\left(W^{P}\right)^{s}$ such that

$$
\left[X_{w_{1}}^{P}\right] \cdots\left[X_{w_{s}}^{P}\right]=d\left[X_{e}^{P}\right] \text { for some } d \neq 0
$$

the following inequality holds:

$$
\omega_{P}\left(\sum_{j=1}^{s} w_{j}^{-1} h_{j}\right) \leq 0 .
$$

Proof. Observe first that, under the identification of $\mathfrak{h}$ with $\mathfrak{h}^{*}$ induced from the Killing form, $\mathfrak{h}_{+}$is isomorphic with the set

$$
\Lambda_{+}(\mathbb{R}):=\left\{\lambda \in \mathfrak{h}^{*}: \lambda\left(\alpha_{i}^{\vee}\right) \in \mathbb{R}_{+}, \text {for all the simple roots } \alpha_{i}\right\}
$$

of dominant real weights in $\mathfrak{h}^{*}$. In fact, under this identification, $x_{j}$ corresponds with $2 \omega_{j} /\left\langle\alpha_{j}, \alpha_{j}\right\rangle$, where $\omega_{j}$ denotes the $j$ th fundamental weight. Thus, the corollary follows from Theorems 10 and 5 .

The same proof as above of Theorem 10 gives the following result, which is weaker in the direction ' $(\mathrm{a}) \Leftarrow(\mathrm{b})$ ' and stronger in the ' $(\mathrm{a}) \Rightarrow(\mathrm{b})$ ' direction.

Theorem 12. For $\lambda_{1}, \ldots, \lambda_{s} \in \Lambda_{+}$, the following are equivalent:

(a) $\left(\lambda_{1}, \ldots, \lambda_{s}\right) \in \Gamma_{s}(G)$.

(b) For any (not necessarily maximal) parabolic subgroup $Q$ and any $w_{1}, \ldots, w_{s}$ $\in W^{Q}$ such that $\left[X_{w_{1}}^{Q}\right] \ldots\left[X_{w_{s}}^{Q}\right] \neq 0$ (not necessarily in the top cohomology class ), the following inequality holds for any maximal parabolic $P \supset Q$ :

$$
I_{\left(w_{1}, \ldots, w_{s}\right)}^{P}: \quad \sum_{j=1}^{s} \lambda_{j}\left(w_{j} x_{P}\right) \leq 0 .
$$

Remark 1. (a) Following Theorem 12, we can easily see that Corollary 11 remains true if we replace (b) (in Corollary 11) by demanding the inequalities (15) for any (not necessarily maximal) parabolic subgroup $Q$ and any $w_{1}, \ldots, w_{s} \in W^{Q}$ such that $\left[X_{w_{1}}^{Q}\right] \ldots\left[X_{w_{s}}^{Q}\right] \neq 0$.

(b) As proved by Belkale $\left[\mathrm{B}_{1}\right]$ for $G=\operatorname{SL}(n)$ and extended for an arbitrary $G$ by Kapovich-Leeb-Millson $\left[\mathrm{KLM}_{1}\right]$, Theorem 10 (and hence Corollary 11) remains true if we replace $d$ by 1 in the identity (12). A much sharper (and optimal) result for an arbitrary $G$ is obtained in Theorem 24 .

\section{Specialization of Theorem 10 to $G=\mathrm{SL}(n)$ : Horn Inequalities}

We first need to recall the Knutson-Tao saturation theorem [KT], conjectured by Zelevinsky [Z]. Other proofs of their result are given by Derksen-Weyman [DK], Belkale $\left[\mathrm{B}_{3}\right]$ and Kapovich-Millson $\left[\mathrm{KM}_{2}\right]$. 
Theorem 13. Let $G=\operatorname{SL}(n)$ and let $\left(\lambda_{1}, \ldots, \lambda_{s}\right) \in \Gamma_{s}(G)$ be such that $\lambda_{1}+\cdots+$ $\lambda_{s}$ belongs to the root lattice. Then,

$$
\left[V\left(\lambda_{1}\right) \otimes \cdots \otimes V\left(\lambda_{s}\right)\right]^{G} \neq 0 .
$$

Specializing Theorem 10 to $G=\operatorname{SL}(n)$, as seen below, we obtain the classical Horn inequalities.

In this case, the partial flag varieties corresponding to the maximal parabolics $P_{r}$ are precisely the Grassmannians of $r$-planes in $n$-space $G / P_{r}=\operatorname{Gr}(r, n)$, for $0<r<n$. The Schubert cells in $\operatorname{Gr}(r, n)$ are parameterized by the subsets of cardinality $r$ :

$$
I=\left\{i_{1}<\ldots<i_{r}\right\} \subset\{1, \ldots, n\} .
$$

The corresponding Weyl group element $w_{I} \in W^{P_{r}}$ is nothing but the permutation

$$
1 \mapsto i_{1}, \quad 2 \mapsto i_{2}, \cdots, r \mapsto i_{r}
$$

and $w_{I}(r+1), \ldots, w_{I}(n)$ are the elements in $\{1, \ldots, n\} \backslash I$ arranged in ascending order.

Let $I$ ' be the 'dual' set

$$
I^{\prime}=\{n+1-i, \quad i \in I\}
$$

arranged in ascending order.

Then, the Schubert class $\left[X_{I}:=X_{w_{I}}^{P_{r}}\right]$ is Poincaré dual to the Schubert class $\left[X_{I^{\prime}}\right] \in H^{*}(\operatorname{Gr}(r, n), \mathbb{Z})$. Moreover,

$$
\operatorname{dim} X_{I}=\operatorname{codim} X_{I^{\prime}}=\left(\sum_{i \in I} i\right)-\frac{r(r+1)}{2} .
$$

We recall the following definition due to Horn.

Definition 2. For $0<r<n$, inductively define the set $S_{n}^{r}$ of triples $(I, J, K)$ of subsets of $\{1, \ldots, n\}$ of cardinality $r$ (arranged in ascending order) satisfying

(a) $\sum_{i \in I} i+\sum_{j \in J} j=\frac{r(r+1)}{2}+\sum_{k \in K} k$.

(b) For all $0<p<r$ and $(F, G, H) \in S_{r}^{p}$, the following inequality holds:

$$
\sum_{f \in F} i_{f}+\sum_{g \in G} j_{g} \leq \frac{p(p+1)}{2}+\sum_{h \in H} k_{h}
$$

The following theorem follows by Theorem 10 for $G=\mathrm{SL}(n)$ (proved by Klyachko) and Theorem 13 (proved by Knutson-Tao). Belkale $\left[\mathrm{B}_{3}\right]$ gave another geometric proof of the theorem.

Theorem 14. For subsets $(I, J, K)$ of $\{1, \ldots, n\}$ of cardinality $r$, the product

$$
\left[X_{I^{\prime}}\right] \cdot\left[X_{J^{\prime}}\right] \cdot\left[X_{K}\right]=d\left[X_{e}^{P_{r}}\right] \text { for some } d \neq 0 \Longleftrightarrow(I, J, K) \in S_{n}^{r} .
$$


Proof. For $\mathrm{SL}(n) / P_{r}=\mathrm{Gr}(r, n)$,

$$
x_{P_{r}}=\epsilon_{1}+\cdots+\epsilon_{r}-\frac{r}{n}\left(\epsilon_{1}+\cdots+\epsilon_{n}\right),
$$

where $\epsilon_{i}$ is the $n \times n$ diagonal matrix with 1 in the $i$ th place and 0 elsewhere. Thus, for $I=\left\{i_{1}<\ldots<i_{r}\right\}$,

$$
w_{I}\left(x_{P_{r}}\right)=\epsilon_{i_{1}}+\cdots+\epsilon_{i_{r}}-\frac{r}{n}\left(\epsilon_{1}+\cdots+\epsilon_{n}\right) .
$$

Recall the classical result that the tensor product structure constants of $\mathrm{GL}_{r^{-}}$ polynomial representations with highest weights

$$
\lambda: n-r \geq \lambda_{1} \geq \lambda_{2} \geq \ldots \geq \lambda_{r} \geq 0
$$

correspond to the intersection product structure constants for the Schubert varieties $X_{I_{\lambda}^{\prime}} \subset \mathrm{SL}(n) / P_{r}=\operatorname{Gr}(r, n)$, where $I_{\lambda} \subset\{1, \ldots, n\}$ is the sequence: $\lambda_{r}+1<$ $\lambda_{r-1}+2<\ldots<\lambda_{1}+r$. Specifically, for $\lambda^{(1)}, \ldots, \lambda^{(s)}$ satisfying (18) with $\sum_{j=1}^{s} \operatorname{dim} X_{I_{\lambda}^{(j)}}=(n-r) r$, we have (cf., e.g., $\left.\left[\mathrm{F}_{0}, \S 9.4\right]\right)$

$$
\operatorname{dim}\left[V\left(\lambda^{(1)}\right) \otimes \ldots \otimes V\left(\lambda^{(s)}\right)\right]^{\mathrm{SL}(r)}=\text { coeff. of }\left[X_{e}^{P_{r}}\right] \text { in } \prod_{j=1}^{s}\left[X_{I_{\lambda}^{\prime}(j)}\right] .
$$

Proof of Theorem 14 ' $\Rightarrow$ ': Take subsets $I, J, K \subset\{1, \ldots, n\}$ of cardinality $r$ such that

$$
\left[X_{I^{\prime}}\right] \cdot\left[X_{J^{\prime}}\right] \cdot\left[X_{K}\right]=d\left[X_{e}^{P_{r}}\right] \in H^{*}\left(\operatorname{SL}(n) / P_{r}\right) \text { for some constant } d \neq 0 .
$$

From the above, we see that

$$
\operatorname{dim} \operatorname{Gr}(r, n)=\operatorname{codim} X_{I^{\prime}}+\operatorname{codim} X_{J^{\prime}}+\operatorname{codim} X_{K},
$$

which gives, by the identity (16),

$$
\operatorname{dim} X_{K}=\operatorname{dim} X_{I}+\operatorname{dim} X_{J} .
$$

From the identities (16) and (21), the identity (a) follows for $(I, J, K)$.

From the identities (19) and (20), we see that as SL(r)-representations,

$$
\left[V\left(\lambda_{I}\right) \otimes V\left(\lambda_{J}\right) \otimes V\left(\lambda_{K^{\prime}}\right)\right]^{\mathrm{SL}(r)} \neq 0,
$$

where $\lambda_{I}$ is the partition

$$
\lambda_{I}: n-r \geq i_{r}-r \geq i_{r-1}-(r-1) \geq \ldots \geq i_{1}-1 \geq 0 .
$$

Thus,

$$
\left(\lambda_{I}, \lambda_{J}, \lambda_{K^{\prime}}\right) \in \Gamma_{3}(\mathrm{SL}(r)) .
$$


Hence, by Theorem 10 applied to $\mathrm{SL}(r)$, for any maximal parabolic subgroup $P_{p} \subset \mathrm{SL}(r), 0<p<r$, and subsets $F, G, H$ of $\{1, \ldots, r\}$ of cardinality $p$ with

$$
\left[X_{F^{\prime}}\right] \cdot\left[X_{G^{\prime}}\right] \cdot\left[X_{H}\right]=d^{\prime}\left[X_{e}^{P_{p}}\right] \text {, for some } d^{\prime} \neq 0,
$$

we have

$$
\lambda_{I}\left(w_{F^{\prime}} x_{P_{p}}\right)+\lambda_{J}\left(w_{G^{\prime}} x_{P_{p}}\right)+\lambda_{K^{\prime}}\left(w_{H} x_{P_{p}}\right) \leq 0 .
$$

Observe that, from the identity (a) of Definition 2,

$$
\left|\lambda_{I}\right|+\left|\lambda_{J}\right|+\left|\lambda_{K^{\prime}}\right|=(n-r) r
$$

Thus, by the identities (17) and (23),

$$
\sum_{f \in F} i_{f}-\sum_{f \in F} f+\sum_{g \in G} j_{g}-\sum_{g \in G} g-\sum_{h \in H} k_{h}+\sum_{h \in H} h \leq 0,
$$

i.e.,

$$
\sum_{f \in F} i_{f}+\sum_{g \in G} j_{g} \leq \sum_{h \in H} k_{h}+\sum_{f \in F} f+\sum_{g \in G} g-\sum_{h \in H} h=\sum_{h \in H} k_{h}+\frac{p(p+1)}{2},
$$

where the last equality follows from the analogue of the identities (16) and (21) corresponding to the identity (22). Now, by induction, assuming the validity of Theorem 14 for the nonvanishing of cup products in $\mathrm{SL}(r) / P_{p}$ (since $p<r<n$ ), we get that

$$
(F, G, H) \in S_{r}^{p} \Leftrightarrow\left[X_{F^{\prime}}\right] \cdot\left[X_{G^{\prime}}\right] \cdot\left[X_{H}\right]=d^{\prime}\left[X_{e}^{P_{p}}\right], \text { for some } d^{\prime} \neq 0 .
$$

Thus, we get that $(I, J, K) \in S_{n}^{r}$, proving the ' $\Rightarrow$ ' implication.

Conversely, assume that the subsets $(I, J, K)$ each of cordinality $r$ contained in $\{1, \ldots, n\}$ belong to $S_{n}^{r}$. We want to prove that

$$
\left[X_{I^{\prime}}\right] \cdot\left[X_{J^{\prime}}\right] \cdot\left[X_{K}\right]=d\left[X_{e}^{P_{r}}\right], \quad \text { for some } d \neq 0 .
$$

By the identity (19) and the condition (a) of Definition 2, this is equivalent to the nonvanishing $\left[V\left(\lambda_{I}\right) \otimes V\left(\lambda_{J}\right) \otimes V\left(\lambda_{K^{\prime}}\right)\right]^{\mathrm{SL}(r)} \neq 0$. By Theorem 13 for $G=\mathrm{SL}(r)$, the latter is equivalent to $\left(\lambda_{I}, \lambda_{J}, \lambda_{K^{\prime}}\right) \in \Gamma_{3}(\mathrm{SL}(r))$, since $\lambda_{I}+\lambda_{J}+\lambda_{K^{\prime}}$ belongs to the root lattice of SL $(r)$ because of the condition (a) (cf. identity (23)).

By Theorem 10 for $G=\mathrm{SL}(r)$ and by assuming the validity of Theorem 14 by induction on $n,\left(\lambda_{I}, \lambda_{J}, \lambda_{K^{\prime}}\right) \in \Gamma_{3}(\mathrm{SL}(r)) \Longleftrightarrow$ for all maximal parabolic sub groups $P_{p}, 0<p<r$, of $\mathrm{SL}(r)$, and all $(F, G, H) \in S_{r}^{p}$, we have

$$
\lambda_{I}\left(w_{F^{\prime}} x_{P_{p}}\right)+\lambda_{J}\left(w_{G^{\prime}} x_{P_{p}}\right)+\lambda_{K^{\prime}}\left(w_{H} x_{P_{p}}\right) \leq 0,
$$

which is equivalent to the inequality

$$
\sum_{f \in F} i_{f}+\sum_{g \in G} j_{g} \leq \sum_{h \in H} k_{h}+\frac{p(p+1)}{2},
$$

by the previous calculation.

But the last inequality is true by the definition of $S_{n}^{r}$. This proves the theorem. 


\section{SHRAWAN KUMAR}

Remark 2. (1) Belkale-Kumar have given two inductive criteria (though only necessary conditions) to determine when the product of a number of Schubert cohomology classes in any $G / P$ is nonzero. The first criterion is in terms of the characters (cf. $\left.\left[\mathrm{BK}_{1}, \mathrm{Thm} .29\right]\right)$ and the second criterion is in terms of dimension counts (cf. $\left[\mathrm{BK}_{1}\right.$, Thm. 36]).

(2) Purbhoo $[\mathrm{P}]$ has given a criterion (again only a necessary condition) to determine which of the Schubert intersections vanish in terms of a combinatorial recipe called 'root game'. He has a similar recipe to determine the vanishing for branching Schubert calculus.

(3) For any cominuscule flag variety $G / P$, Purbhoo-Sottile have determined a recursive set of inequalities (coming only from the class of cominuscule flag varieties) which determines when the intersection product $\left[X_{w_{1}}^{P}\right] \ldots\left[X_{w_{s}}^{P}\right]$ is nonzero in $H^{*}(G / P)$ (cf. [PS, Thm. 4]).

For a Hermitian $n \times n$ matrix $A$, let $\lambda_{A}=\left(\lambda_{1} \geq \cdots \geq \lambda_{n}\right)$ be its set of eigenvalues (which are all real). Let $\mathfrak{a}$ be the standard Cartan subalgebra of $\operatorname{sl}(n)$ consisting of traceless diagonal matrices and let $\mathfrak{b} \subset \operatorname{sl}(n)$ be the standard Borel subalgebra consisting of traceless upper triangular matrices (where $s l(n)$ is the Lie algebra of $\mathrm{SL}(n)$ consisting of traceless $n \times n$-matrices). Then, the Weyl chamber

$$
\mathfrak{a}_{+}=\left\{\operatorname{diag}\left(e_{1} \geq \cdots \geq e_{n}\right): \sum e_{i}=0\right\} .
$$

Define the Hermitian eigencone

$$
\begin{aligned}
\bar{\Gamma}(n)=\{ & \left(a_{1}, a_{2}, a_{3}\right) \in \mathfrak{a}_{+}^{3}: \text { there exist } n \times n \text { Hermitian matrices } A, B, C \text { with } \\
& \left.\lambda_{A}=a_{1}, \lambda_{B}=a_{2}, \lambda_{C}=a_{3} \text { and } A+B=C\right\} .
\end{aligned}
$$

It is easy to see that $\bar{\Gamma}(n)$ essentially coincides with the eigencone $\bar{\Gamma}_{3}(\operatorname{sl}(n))$ defined in Section 3. Specifically,

$$
\left(a_{1}, a_{2}, a_{3}\right) \in \bar{\Gamma}(n) \Longleftrightarrow\left(a_{1}, a_{2}, a_{3}^{*}\right) \in \bar{\Gamma}_{3}(s l(n)),
$$

where $\left(e_{1} \geq \cdots \geq e_{n}\right)^{*}:=\left(-e_{n} \geq \cdots \geq-e_{1}\right)$.

Combining Corollary 11 with Theorem 14, we get the following Horn's conjecture [Ho] established by the works of Klyachko (Corollary 11 for $\mathfrak{g}=\operatorname{sl}(n)$ ) and Knutson-Tao (Theorem 13). Danilov-Koshevoy $[\mathrm{DK}]^{2}$ have given a solution of a different formulation of Horn's problem in terms of discretely concave functions (without using GIT).

Corollary 15. For $\left(a_{1}, a_{2}, a_{3}\right) \in \mathfrak{a}_{+}^{3}$, the following are equivalent.

(a) $\left(a_{1}, a_{2}, a_{3}\right) \in \bar{\Gamma}(n)$.

(b) For all $0<r<n$ and all $(I, J, K) \in S_{n}^{r}$,

$$
\left|a_{3}(K)\right| \leq\left|a_{1}(I)\right|+\left|a_{2}(J)\right|,
$$

where for a subset $I=\left(i_{1}<\cdots<i_{r}\right) \subset\{1, \ldots, n\}$ and $a=\left(e_{1} \geq \cdots \geq\right.$ $\left.e_{n}\right) \in \mathfrak{a}_{+}, a(I):=\left(e_{i_{1}} \geq \cdots \geq e_{i_{r}}\right)$, and $|a(I)|:=e_{i_{1}}+\cdots+e_{i_{r}}$.

\footnotetext{
${ }^{2}$ We thank them for bringing their paper to our attention.
} 


\section{EIGENVALUE PROBLEM}

Proof. Clearly $\left(a_{1}, a_{2}, a_{3}\right) \in \bar{\Gamma}(n) \Longleftrightarrow\left(a_{1}^{*}, a_{2}^{*}, a_{3}^{*}\right) \in \bar{\Gamma}(n)$. Thus, by Corollary 11 and Theorem 14, (a) is equivalent to the condition that for all $0<r<n$ and $(I, J, K) \in S_{n}^{r}$,

$$
\omega_{P_{r}}\left(w_{I^{\prime}}^{-1} a_{1}^{*}+w_{J^{\prime}}^{-1} a_{2}^{*}+w_{K}^{-1} a_{3}\right) \leq 0 .
$$

Now, since $\omega_{P_{r}}$ corresponds to $x_{P_{r}}$ under the isomorphism of $\mathfrak{a}^{*}$ with $\mathfrak{a}$ induced from the Killing form $\langle$,$\rangle , the inequality (24) is equivalent to$

$$
\left\langle a_{1}^{*}, w_{I^{\prime}} x_{P_{r}}\right\rangle+\left\langle a_{2}^{*}, w_{J^{\prime}} x_{P_{r}}\right\rangle+\left\langle a_{3}, w_{K} x_{P_{r}}\right\rangle \leq 0 .
$$

Now, from the identity (17), the inequality (25) is equivalent to (since trace $a_{1}=$ trace $a_{2}=$ trace $a_{3}^{*}=0$ by assumption):

$$
\left|a_{3}(K)\right| \leq\left|a_{1}(I)\right|+\left|a_{2}(J)\right| .
$$

This proves the corollary.

We have the following representation theoretic analogue of the above corollary, obtained by combining Theorems 10, 13 and 14 .

Corollary 16. Let $\lambda=\left(\lambda_{1} \geq \cdots \geq \lambda_{n} \geq 0\right), \mu=\left(\mu_{1} \geq \cdots \geq \mu_{n} \geq 0\right)$ and $\nu=\left(\nu_{1} \geq \cdots \geq \nu_{n} \geq 0\right)$ be three partitions such that $|\lambda|+|\mu|-|\nu| \in n \mathbb{Z}$, where $|\lambda|:=\lambda_{1}+\cdots+\lambda_{n}$. Then, the following are equivalent:

(a) $V(\nu)$ appears as a $\mathrm{SL}(n)$-submodule of $V(\lambda) \otimes V(\mu)$.

(b) For all $0<r<n$ and all $(I, J, K) \in S_{n}^{r}$,

$$
|\nu(K)| \leq|\lambda(I)|+|\mu(J)|-\frac{r}{n}(|\lambda|+|\mu|-|\nu|),
$$

where for a subset $I=\left(i_{1}<\cdots<i_{r}\right) \subset\{1, \ldots, n\}, \lambda(I)$ denotes $\left(\lambda_{i_{1}} \geq\right.$ $\left.\cdots \geq \lambda_{i_{r}}\right)$ and $|\lambda(I)|:=\lambda_{i_{1}}+\cdots+\lambda_{i_{r}}$.

Proof. The condition $|\lambda|+|\mu|-|\nu| \in n \mathbb{Z}$ is equivalent to the condition that $\lambda+\mu+\nu^{*}$ belongs to the root lattice of $s l(n)$, where $\nu^{*}$ is the partition $\left(\nu_{1}-\nu_{n} \geq \cdots \geq\right.$ $\left.\nu_{1}-\nu_{2} \geq 0 \geq 0\right)$. Moreover, $V(\nu) \subset V(\lambda) \otimes V(\mu)$ (as an $\mathrm{SL}(n)$-submodule) if and only if $V\left(\nu^{*}\right) \subset V\left(\lambda^{*}\right) \otimes V\left(\mu^{*}\right)$ (as an $\mathrm{SL}(n)$-submodule). Thus, by Theorems 10,13 and 14, (a) is equivalent to the condition that for all $0<r<n$ and all $(I, J, K) \in S_{n}^{r}$,

$$
\lambda^{*}\left(w_{I^{\prime}} x_{P_{r}}\right)+\mu^{*}\left(w_{J^{\prime}} x_{P_{r}}\right)+\nu\left(w_{K} x_{P_{r}}\right) \leq 0 .
$$

By using the identity (17), the above inequality (26) is equivalent to

$$
|\nu(K)| \leq|\lambda(I)|+|\mu(J)|-\frac{r}{n}(|\lambda|+|\mu|-|\nu|) .
$$

This proves the corollary.

Definition 3. For $0<r<n$, inductively define $\widehat{S}_{n}^{r}$ as the set of triples $(I, J, K)$, where $I, J, K$ are subsets of $\{1, \ldots, n\}$ of cardinality $r$ satisfying the condition (b) of Definition 2 for $\widehat{S}_{r}^{p}$ and the condition (a') (instead of the condition (a)).

$$
\sum_{i \in I} i+\sum_{j \in J} j \leq \frac{r(r+1)}{2}+\sum_{k \in K} k .
$$

The following result is due to Belkale $\left[\mathrm{B}_{3}\right.$, Thm. 0.1], which is parallel to Theorem 14. 


\section{SHRAWAN KUMAR}

Theorem 17. Let $0<r<n$. For subsets $(I, J, K)$ of $\{1, \ldots, n\}$ of cardinality $r$, the product

$$
\left[X_{I^{\prime}}\right] \cdot\left[X_{J^{\prime}}\right] \cdot\left[X_{K}\right] \quad \text { is nonzero } \Leftrightarrow \quad(I, J, K) \in \widehat{S}_{n}^{r} \text {. }
$$

Remark 3. The Hermitian eigencone $\bar{\Gamma}(n)$ has extensively been studied since the initial work of H. Weyl in 1912 [W] followed by the works of Fan [Fa], Lidskii [Li], Wielandt [Wi] and culminating in the conjecture of Horn [Ho] and then its proof by combining the works of Klyachko [Kly] and Knutson-Tao [KT]. (Also see Thompson-Freede [TF].) For a detailed survey on the subject, we refer to Fulton's article $\left[\mathrm{F}_{2}\right]$.

\section{Deformed product}

This section is based on the work $\left[\mathrm{BK}_{1}\right]$ due to Belkale-Kumar.

We continue to follow the notation and assumptions from Secton 2; in particular, $G$ is a semisimple connected complex algebraic group and $P \subset G$ is a standard parabolic subgroup.

Consider the shifted Bruhat cell:

$$
\Phi_{w}^{P}:=w^{-1} B w P \subset G / P .
$$

Let $T^{P}=T(G / P)_{e}$ be the tangent space of $G / P$ at $e \in G / P$. It carries a canonical action of $P$. For $w \in W^{P}$, define $T_{w}^{P}$ to be the tangent space of $\Phi_{w}^{P}$ at $e$. We shall abbreviate $T^{P}$ and $T_{w}^{P}$ by $T$ and $T_{w}$ respectively when the reference to $P$ is clear. By (8), $B_{L}$ stabilizes $\Phi_{w}^{P}$ keeping $e$ fixed. Thus,

$$
B_{L} T_{w} \subset T_{w} .
$$

The following result follows easily from the Kleiman transversality theorem Theorem 6 and Proposition 7 by observing that $g \Phi_{w}^{P}$ passes through $e \Leftrightarrow g \Phi_{w}^{P}=p \Phi_{w}^{P}$ for some $p \in P$.

Proposition 18. Take any $\left(w_{1}, \ldots, w_{s}\right) \in\left(W^{P}\right)^{s}$ such that

$$
\sum_{j=1}^{s} \operatorname{codim} \Phi_{w_{j}}^{P} \leq \operatorname{dim} G / P
$$

Then, the following three conditions are equivalent:

(a) $\left[X_{w_{1}}^{P}\right] \ldots\left[X_{w_{s}}^{P}\right] \neq 0 \in H^{*}(G / P)$.

(b) For general $\left(p_{1}, \ldots, p_{s}\right) \in P^{s}$, the intersection $p_{1} \Phi_{w_{1}}^{P} \cap \cdots \cap p_{s} \Phi_{w_{s}}^{P}$ is transverse at $e$.

(c) For general $\left(p_{1}, \ldots, p_{s}\right) \in P^{s}$,

$$
\operatorname{dim}\left(p_{1} T_{w_{1}} \cap \cdots \cap p_{s} T_{w_{s}}\right)=\operatorname{dim} G / P-\sum_{j=1}^{s} \operatorname{codim} \Phi_{w_{j}}^{P} .
$$

The set of s-tuples in (b) as well as (c) is an open subset of $P^{s}$. 
Definition 4. Let $w_{1}, \ldots, w_{s} \in W^{P}$ be such that

$$
\sum_{j=1}^{s} \operatorname{codim} \Phi_{w_{j}}^{P}=\operatorname{dim} G / P
$$

We then call the $s$-tuple $\left(w_{1}, \ldots, w_{s}\right)$ Levi-movable (for short, L-movable) if, for general $\left(l_{1}, \ldots, l_{s}\right) \in L^{s}$, the intersection $l_{1} \Phi_{w_{1}}^{P} \cap \cdots \cap l_{s} \Phi_{w_{s}}^{P}$ is transverse at $e$.

By Proposition 18 , if $\left(w_{1}, \ldots, w_{s}\right)$ is $L$-movable, then $\left[X_{w_{1}}^{P}\right] \ldots\left[X_{w_{s}}^{P}\right]=d\left[X_{e}^{P}\right]$ in $H^{*}(G / P)$, for some nonzero $d$.

Definition 5. Let $w \in W^{P}$. Since $T_{w}$ is a $B_{L}$-module (by (27)), we have the $P$-equivariant vector bundle $\mathcal{T}_{w}:=P \times T_{w}$ on $P / B_{L}$. In particular, we have the $P$-equivariant vector bundle $\mathcal{T}:=P \underset{B_{L}}{\stackrel{B_{L}}{\times}} T$ and $\mathcal{T}_{w}$ is canonically a $P$-equivariant subbundle of $\mathcal{T}$. Take the top exterior powers $\operatorname{det}\left(\mathcal{T} / \mathcal{T}_{w}\right)$ and $\operatorname{det}\left(\mathcal{T}_{w}\right)$, which are $P$-equivariant line bundles on $P / B_{L}$. Observe that, since $T$ is a $P$-module, the $P$ equivariant vector bundle $\mathcal{T}$ is $P$-equivariantly isomorphic with the product bundle $P / B_{L} \times T$ under the map $\xi: P / B_{L} \times T \rightarrow \mathcal{T}$ taking $\left(p B_{L}, v\right) \mapsto\left[p, p^{-1} v\right]$, for $p \in P$ and $v \in T$; where $P$ acts on $P / B_{L} \times T$ diagonally. We will often identify $\mathcal{T}$ with the product bundle $P / B_{L} \times T$ under $\xi$.

For $w \in W^{P}$, define the character $\chi_{w} \in \Lambda$ by

$$
\chi_{w}=\sum_{\beta \in\left(R^{+} \backslash R_{\mathfrak{\imath}}^{+}\right) \cap w^{-1} R^{+}} \beta .
$$

Then, from $\left[\mathrm{K}_{1}, 1.3 .22 .3\right]$ and $(8)$,

$$
\chi_{w}=\rho-2 \rho^{L}+w^{-1} \rho,
$$

where $\rho$ (resp. $\rho^{L}$ ) is half the sum of the roots in $R^{+}$(resp. in $R_{\mathfrak{l}}^{+}$).

The following lemma is easy to establish.

Lemma 19. For $w \in W^{P}$, as $P$-equivariant line bundles on $P / B_{L}$, we have: $\operatorname{det}\left(\mathcal{T} / \mathcal{T}_{w}\right)=\mathcal{L}_{P}\left(\chi_{w}\right)$.

Let $\mathcal{T}_{s}$ be the $P$-equivariant product bundle $\left(P / B_{L}\right)^{s} \times T \rightarrow\left(P / B_{L}\right)^{s}$ under the diagonal action of $P$ on $\left(P / B_{L}\right)^{s} \times T$. Then, $\mathcal{T}_{s}$ is canonically $P$-equivariantly isomorphic with the pull-back bundle $\pi_{j}^{*}(\mathcal{T})$, for any $1 \leq j \leq s$, where $\pi_{j}$ : $\left(P / B_{L}\right)^{s} \rightarrow P / B_{L}$ is the projection onto the $j$ th factor. For any $w_{1}, \ldots, w_{s} \in W^{P}$, we have a $P$-equivariant map of vector bundles on $\left(P / B_{L}\right)^{s}$ :

$$
\Theta=\Theta_{\left(w_{1}, \ldots, w_{s}\right)}: \mathcal{T}_{s} \rightarrow \bigoplus_{j=1}^{s} \pi_{j}^{*}\left(\mathcal{T} / \mathcal{T}_{w_{j}}\right)
$$

obtained as the direct sum of the projections $\mathcal{T}_{s} \rightarrow \pi_{j}^{*}\left(\mathcal{T} / \mathcal{T}_{w_{j}}\right)$ under the identification $\mathcal{T}_{s} \simeq \pi_{j}^{*}(\mathcal{T})$. Now, assume that $w_{1}, \ldots, w_{s} \in W^{P}$ satisfies the condition 


\section{SHRAWAN KUMAR}

(29). In this case, we have the same rank bundles on the two sides of the map (32). Let $\theta$ be the bundle map obtained from $\Theta$ by taking the top exterior power:

$$
\theta=\operatorname{det}(\Theta): \operatorname{det}\left(\mathcal{T}_{s}\right) \rightarrow \operatorname{det}\left(\mathcal{T} / \mathcal{T}_{w_{1}}\right) \otimes \cdots \otimes \operatorname{det}\left(\mathcal{T} / \mathcal{T}_{w_{s}}\right)
$$

Clearly, $\theta$ is $P$-equivariant and hence one can view $\theta$ as a $P$-invariant element in

$$
\begin{aligned}
& H^{0}\left(\left(P / B_{L}\right)^{s}, \operatorname{det}\left(\mathcal{T}_{s}\right)^{*} \otimes\left(\operatorname{det}\left(\mathcal{T} / \mathcal{T}_{w_{1}}\right) \otimes \cdots \otimes \operatorname{det}\left(\mathcal{T} / \mathcal{T}_{w_{s}}\right)\right)\right) \\
& \quad=H^{0}\left(\left(P / B_{L}\right)^{s}, \mathbb{L}\right), \text { where } \mathbb{L}:=\mathcal{L}_{P}\left(\chi_{w_{1}}-\chi_{1}\right) \otimes \cdots \otimes \mathcal{L}_{P}\left(\chi_{w_{s}}\right) .
\end{aligned}
$$

The following lemma follows easily from Proposition 18.

Lemma 20. Let $\left(w_{1}, \ldots, w_{s}\right)$ be an s-tuple of elements of $W^{P}$ satisfying the condition (29). Then we have the following:

(a) The section $\theta$ is nonzero if and only if $\left[X_{w_{1}}^{P}\right] \ldots\left[X_{w_{s}}^{P}\right] \neq 0 \in H^{*}(G / P)$.

(b) The $s$-tuple $\left(w_{1}, \ldots, w_{s}\right)$ is L-movable if and only if the section $\theta$ restricted to $\left(L / B_{L}\right)^{s}$ is not identically 0 .

Proposition 21. Assume that $\left(w_{1}, \ldots, w_{s}\right) \in\left(W^{P}\right)^{s}$ satisfies equation (29). Then the following are equivalent:

(a) $\left(w_{1}, \ldots, w_{s}\right)$ is L-movable.

(b) $\left[X_{w_{1}}^{P}\right] \ldots\left[X_{w_{s}}^{P}\right]=d\left[X_{e}^{P}\right]$ in $H^{*}(G / P)$, for some nonzero $d$, and for each $\alpha_{i} \in \Delta \backslash \Delta(P)$, we have

$$
\left(\left(\sum_{j=1}^{s} \chi_{w_{j}}\right)-\chi_{1}\right)\left(x_{i}\right)=0
$$

Proof. $(\mathrm{a}) \Rightarrow(\mathrm{b})$ : Let $\left(w_{1}, \ldots, w_{s}\right) \in\left(W^{P}\right)^{s}$ be $L$-movable. Consider the restriction $\widehat{\theta}$ of the $P$-invariant section $\theta$ to $\left(L / B_{L}\right)^{s}$. Then, $\widehat{\theta}$ is nonvanishing by the above lemma. But, for

$$
H^{0}\left(\left(L / B_{L}\right)^{s}, \mathbb{L}\right)^{L}
$$

to be nonzero, the center of $L$ should act trivially (under the diagonal action) on $\mathbb{L}$ restricted to $\left(L / B_{L}\right)^{s}$, where $\mathbb{L}$ is as in the identity (34). This gives $\sum_{j=1}^{s} \chi_{w_{j}}(h)=$ $\chi_{1}(h)$, for all $h$ in the Lie algebra $\mathfrak{z}_{L}$ of the center of $L$; in particular, for $h=x_{i}$ for $\alpha_{i} \in \Delta \backslash \Delta(P)$. Further, the assertion that $\left[X_{w_{1}}^{P}\right] \ldots\left[X_{w_{s}}^{P}\right]=d\left[X_{e}^{P}\right]$, for some nonzero $d$, follows from Proposition 18 and the condition (29).

$(\mathrm{b}) \Rightarrow(\mathrm{a})$ : By the above lemma, $\theta\left(\bar{p}_{1}, \ldots, \bar{p}_{s}\right) \neq 0$, for some $\bar{p}_{j} \in P / B_{L}$. Consider the central OPS of $L: \delta(t):=\prod_{\alpha_{i} \in \Delta \backslash \Delta(P)} t^{\bar{x}_{i}}$, where $\bar{x}_{i}$ is the smallest positive integral multiple of $x_{i}$ such that $\bar{x}_{i}$ lies in the coroot lattice of $\mathfrak{g}$. For any $x=$ $u l B_{L} \in P / B_{L}$, with $u \in U_{P}$ and $l \in L_{P}$,

$$
\lim _{t \rightarrow 0} \delta(t) x=\lim _{t \rightarrow 0} \delta(t) u \delta(t)^{-1}(\delta(t) l) B_{L}
$$

But, since $\beta(\dot{\delta})>0$, for all $\beta \in R^{+} \backslash R_{\mathfrak{l}}^{+}$, we get $\lim _{t \rightarrow 0} \delta(t) u \delta(t)^{-1}=1$. Moreover, since $\delta(t)$ is central in $L, \delta(t) l B_{L}=l B_{L}$; in particular, the limit $\lim _{t \rightarrow 0} \delta(t) l B_{L}$ exists and equals $l B_{L}$. Thus, $\lim _{t \rightarrow 0} \delta(t) x$ exists and lies in $L / B_{L}$. 
Let $\bar{p}:=\left(\bar{p}_{1}, \ldots, \bar{p}_{s}\right) \in \mathbb{X}:=\left(P / B_{L}\right)^{s}$. Then, by Lemma 9 (since $\delta$ is central in $L)$, we get

$$
\mu^{\mathbb{L}}(\bar{p}, \delta)=-\sum_{\alpha_{i} \in \Delta \backslash \Delta(P)}\left(\left(\left(\sum_{j=1}^{s} \chi_{w_{j}}\right)-\chi_{1}\right)\left(\bar{x}_{i}\right)\right)
$$

$=0$, by assumption.

Therefore, using Proposition 8 (c) for $S=P, \theta$ does not vanish at $\lim _{t \rightarrow 0} \delta(t) \bar{p}$. But, from the above, this limit exists as an element of $\left(L / B_{L}\right)^{s}$. Hence, $\left(w_{1}, \ldots, w_{s}\right)$ is $L$-movable by Lemma 20 .

Corollary 22. For any $u, v, w \in W^{P}$ such that $c_{u, v}^{w} \neq 0$ (cf. equation (36)), we have

$$
\left(\chi_{w}-\chi_{u}-\chi_{v}\right)\left(x_{i}\right) \geq 0 \text { for each } \alpha_{i} \in \Delta \backslash \Delta(P) .
$$

Proof. By the assumption of the corollary and the identity $(9),\left[X_{u}^{P}\right] \cdot\left[X_{v}^{P}\right]$. $\left[X_{w_{o} w w_{o}^{P}}^{P}\right]=d\left[X_{e}^{P}\right]$, for some nonzero $d$ (in fact $d=c_{u, v}^{w}$ ). Thus, by taking $\left(w_{1}, w_{2}, w_{3}\right)=\left(u, v, w_{o} w w_{o}^{P}\right)$ in Lemma 20 , the section $\theta$ is nonzero. Now, apply Proposition $8(\mathrm{~b})$ for the OPS $\delta(t)=t^{\bar{x}_{i}}$ and Lemma 9 (together with the identity (31)) to get the corollary.

The definition of the following deformed product $\odot_{0}$ (now known as the BelkaleKumar product) was arrived at from the crucial concept of Levi-movability as in Definition 4 . This deformed product is used in determining the facets (codimension 1 faces) of $\bar{\Gamma}_{s}(\mathfrak{g})$.

Definition 6. Let $P$ be any standard parabolic subgroup of $G$. Write the standard cup product in $H^{*}(G / P, \mathbb{Z})$ in the $\left\{\left[X_{w}^{P}\right]\right\}_{w \in W^{P}}$ basis as follows:

$$
\left[X_{u}^{P}\right] \cdot\left[X_{v}^{P}\right]=\sum_{w \in W^{P}} c_{u, v}^{w}\left[X_{w}^{P}\right]
$$

Introduce the indeterminates $\tau_{i}$ for each $\alpha_{i} \in \Delta \backslash \Delta(P)$ and define a deformed cup product $\odot$ as follows:

$$
\left[X_{u}^{P}\right] \odot\left[X_{v}^{P}\right]=\sum_{w \in W^{P}}\left(\prod_{\alpha_{i} \in \Delta \backslash \Delta(P)} \tau_{i}^{\left(w^{-1} \rho-u^{-1} \rho-v^{-1} \rho-\rho\right)\left(x_{i}\right)}\right) c_{u, v}^{w}\left[X_{w}^{P}\right],
$$

where $\rho$ is the (usual) half sum of positive roots of $\mathfrak{g}$.

By Corollary 22 and the identity (31), whenever $c_{u, v}^{w}$ is nonzero, the exponent of $\tau_{i}$ in the above is a nonnegative integer. Moreover, it is easy to see that the product $\odot$ is associative and clearly commutative. This product should not be confused with the small quantum cohomology product of $G / P$.

The cohomology of $G / P$ obtained by setting each $\tau_{i}=0$ in $\left(H^{*}(G / P, \mathbb{Z}) \otimes\right.$ $\left.\mathbb{Z}\left[\tau_{i}\right], \odot\right)$ is denoted by $\left(H^{*}(G / P, \mathbb{Z}), \odot_{0}\right)$. Thus, as a $\mathbb{Z}$-module, it is the same as the singular cohomology $H^{*}(G / P, \mathbb{Z})$ and under the product $\odot_{0}$ it is associative (and commutative). Moreover, it continues to satisfy the Poincaré duality (cf. [ $\mathrm{BK}_{1}$, Lemma 16(d)]). 


\section{SHRAWAN KUMAR}

It should be remarked that, in general, the canonical pull-back map $H^{*}\left(G / P_{2}, \mathbb{Z}\right)$ $\rightarrow H^{*}\left(G / P_{1}, \mathbb{Z}\right)$, for $P_{1} \subset P_{2}$, does not respect the product $\odot_{0}$.

In the $\left\{\epsilon_{w}^{P}\right\}_{w \in W^{P}}$ basis, by the identity (9), the deformed product takes the form

$$
\epsilon_{u}^{P} \odot \epsilon_{v}^{P}=\sum_{w \in W^{P}}\left(\prod_{\alpha_{i} \in \Delta \backslash \Delta(P)} \tau_{i}^{\left(u^{-1} \rho+v^{-1} \rho-w^{-1} \rho-\rho\right)\left(x_{i}\right)}\right) d_{u, v}^{w} \epsilon_{w}^{P},
$$

where $\epsilon_{u}^{P} \cdot \epsilon_{v}^{P}=\sum_{w \in W^{P}} d_{u, v}^{w} \epsilon_{w}^{P}$.

Lemma 23. Let $P$ be a cominuscule maximal standard parabolic subgroup of $G$ (i.e., the unique simple root $\alpha_{P} \in \Delta \backslash \Delta(P)$ appears with coefficient 1 in the highest root of $\left.R^{+}\right)$. Then, the product $\odot$ coincides with the cup product in $H^{*}(G / P)$.

Proof. By the definition of $\odot$, it suffices to show that for any $u, v, w \in W^{P}$ such that $c_{u, v}^{w} \neq 0$,

$$
\left(\chi_{w}-\left(\chi_{u}+\chi_{v}\right)\right)\left(x_{P}\right)=0 .
$$

By the definition of $\chi_{w}$ (cf. (30)), since $P$ is cominuscule,

$$
\chi_{w}\left(x_{P}\right)=\left|w^{-1} R^{+} \cap\left(R^{+} \backslash R_{\mathfrak{l}}^{+}\right)\right|=\operatorname{codim}\left(\Phi_{w}^{P}: G / P\right),
$$

where the last equality follows since

$$
R\left(T_{w}\right)=w^{-1} R^{+} \cap\left(R^{-} \backslash R_{\mathfrak{l}}^{-}\right)
$$

where $R^{-}:=R \backslash R^{+}$and $R_{\mathfrak{l}}^{-}:=R_{\mathfrak{l}} \backslash R_{\mathfrak{l}}^{+}$. Moreover, since $c_{u, v}^{w} \neq 0$,

$$
\operatorname{codim}\left(\Phi_{u}^{P}: G / P\right)+\operatorname{codim}\left(\Phi_{v}^{P}: G / P\right)=\operatorname{codim}\left(\Phi_{w}^{P}: G / P\right) .
$$

Combining equations (39) and (40), we get equation (38).

Alternatively, one can prove the lemma by observing that the unipotent radical $U_{P}$ of $P$ acts trivially on the tangent space $T_{P}(G / P)$ and by using the definition of Levi-movability together with Proposition 18.

Remark 4. Belkale-Kumar have given a criterion (though only necessary conditions) to determine when the deformed product of a number of Schubert cohomology classes in any $G / P$ is nonzero. The criterion is in terms of the characters (cf. $\left[\mathrm{BK}_{1}\right.$, Theorem 32]).

\section{Efficient determination of the eigencone}

This section is again based on the work $\left[\mathrm{BK}_{1}\right]$ due to Belkale-Kumar. The following theorem $\left[\mathrm{BK}_{1}\right.$, Thm. 22] determines the saturated tensor semigroup $\Gamma_{s}(G)$ efficiently. Specifically, as proved by Ressayre (see Corollary 36), the set of inequalities given by (b) of the following theorem (resp. (b) of Corollary 29) is an irredundant set of inequalities determining $\Gamma_{s}(G)\left(\operatorname{resp} . \bar{\Gamma}_{s}(\mathfrak{g})\right)$.

For $G=\operatorname{SL}(n)$, each maximal parabolic $P$ is cominuscule, and hence, by Lemma $23, \odot_{0}$ coincides with the standard cup product in $H^{*}(G / P)$. Thus, the following theorem (resp. Corollary 29) in this case reduces to Theorem 10 (resp. Corollary 


\section{EIGENVALUE PROBLEM}

11) with $d=1$ in the identity (12). In this case the irredundancy of the system was proved by Knutson-Tao-Woodward [KTW].

It may be mentioned that replacing the product $\odot_{0}$ in the (b)-part of the following theorem by the standard cup product (i.e., Theorem 10 with $d=1$ in the identity (12); cf. Remark 1 (b)), we get, in general, 'far more' inequalities for simple groups other than $\mathrm{SL}(n)$. For example, for $G$ of type $\mathrm{B}_{3}$ (or $\mathrm{C}_{3}$ ), Theorem 10 with $d=1$ gives rise to 126 inequalities, whereas the following theorem gives only 93 inequalities (cf. [KuLM]).

Theorem 24. Let $G$ be a connected semisimple group and let $\left(\lambda_{1}, \ldots, \lambda_{s}\right) \in \Lambda_{+}^{s}$. Then, the following are equivalent:

(a) $\lambda=\left(\lambda_{1}, \ldots, \lambda_{s}\right) \in \Gamma_{s}(G)$.

(b) For every standard maximal parabolic subgroup $P$ in $G$ and every choice of s-tuples $\left(w_{1}, \ldots, w_{s}\right) \in\left(W^{P}\right)^{s}$ such that

$$
\left[X_{w_{1}}^{P}\right] \odot_{0} \cdots \odot_{0}\left[X_{w_{s}}^{P}\right]=\left[X_{e}^{P}\right] \in\left(H^{*}(G / P, \mathbb{Z}), \odot_{0}\right),
$$

the following inequality holds:

$$
\sum_{j=1}^{s} \lambda_{j}\left(w_{j} x_{P}\right) \leq 0, \quad \quad\left(I_{\left(w_{1}, \ldots, w_{s}\right)}^{P}\right)
$$

where $\alpha_{i_{P}}$ is the (unique) simple root in $\Delta \backslash \Delta(P)$ and $x_{P}:=x_{i_{P}}$.

Before we come to the proof of the theorem, we need the following.

Definition 7. (Maximally destabilizing one-parameter subgroups) We recall the definition of Kempf's OPS attached to an unstable point, which is in some sense the 'most destabilizing' OPS. Let $\mathbb{X}$ be a projective variety with the action of a connected reductive group $S$ and let $\mathbb{L}$ be an $S$-linearized ample line bundle on $\mathbb{X}$. Introduce the set $M(S)$ of fractional OPS in $S$. This is the set consisting of the ordered pairs $(\delta, a)$, where $\delta \in O(S)$ and $a \in \mathbb{Z}_{>0}$, modulo the equivalence relation $(\delta, a) \simeq(\gamma, b)$ if $\delta^{b}=\gamma^{a}$. The equivalence class of $(\delta, a)$ is denoted by $[\delta, a]$. An OPS $\delta$ of $S$ can be thought of as the element $[\delta, 1] \in M(S)$. The group $S$ acts on $M(S)$ via conjugation: $g \cdot[\delta, a]=\left[g \delta g^{-1}, a\right]$. Choose an $S$-invariant norm $q: M(S) \rightarrow \mathbb{R}_{+}$, where norm means that $q_{\left.\right|_{M(H)}}$ is the square root of a positive definite quadratic form on the $\mathbb{Q}$-vector space $M(H)$ for a maximal torus $H$ of $S$. We can extend the definition of $\mu^{\mathbb{L}}(x, \delta)$ to any element $\widehat{\delta}=[\delta, a] \in M(S)$ and $x \in \mathbb{X}$ by setting $\mu^{\mathbb{L}}(x, \widehat{\delta})=\mu^{\mathbb{L}}(x, \delta) / a$. We note the following elementary property: If $\widehat{\delta} \in M(S)$ and $p \in P(\delta)$ (where $P(\delta)$ is the Kempf's parabolic defined by the identity (11)), then

$$
\mu^{\mathbb{L}}(x, \widehat{\delta})=\mu^{\mathbb{L}}\left(x, p \widehat{\delta} p^{-1}\right) .
$$

For any unstable (i.e., nonsemistable) point $x \in \mathbb{X}$, define

$$
q^{*}(x)=\inf _{\widehat{\delta} \in M(S)}\left\{q(\widehat{\delta}) \mid \mu^{\mathbb{L}}(x, \widehat{\delta}) \leq-1\right\},
$$


and the optimal class

$$
\Lambda(x)=\left\{\widehat{\delta} \in M(S) \mid \mu^{\mathbb{L}}(x, \widehat{\delta}) \leq-1, q(\widehat{\delta})=q^{*}(x)\right\} .
$$

Any $\widehat{\delta} \in \Lambda(x)$ is called Kempf's OPS associated to $x$.

By a theorem of Kempf (cf. [Ki, Lemma 12.13]), $\Lambda(x)$ is nonempty and the parabolic $P(\widehat{\delta}):=P(\delta)$ (for $\widehat{\delta}=[\delta, a]$ ) does not depend upon the choice of $\widehat{\delta} \in \Lambda(x)$. The parabolic $P(\widehat{\delta})$ for $\widehat{\delta} \in \Lambda(x)$ will be denoted by $P(x)$ and called the Kempf's parabolic associated to the unstable point $x$. Moreover, $\Lambda(x)$ is a single conjugacy class under $P(x)$.

We recall the following theorem due to Ramanan-Ramanathan [RR, Prop. 1.9].

Theorem 25. For any unstable point $x \in \mathbb{X}$ and $\widehat{\delta}=[\delta, a] \in \Lambda(x)$, let

$$
x_{o}=\lim _{t \rightarrow 0} \delta(t) \cdot x \in \mathbb{X}
$$

Then, $x_{o}$ is unstable and $\widehat{\delta} \in \Lambda\left(x_{o}\right)$.

For a real number $d>0$ and $\widehat{\delta}=[\delta, a] \in M(S)$, define

$$
\mathbb{X}_{d, \widehat{\delta}}=\mathbb{X}_{d, \widehat{\delta}}^{\mathbb{L}}:=\left\{x \in \mathbb{X}: q^{*}(x)=d \text { and } \widehat{\delta} \in \Lambda(x)\right\}
$$

and

$$
Z_{d, \widehat{\delta}}=Z_{d, \widehat{\delta}}^{\mathbb{L}}:=\left\{x \in \mathbb{X}_{d, \widehat{\delta}}: \delta \text { fixes } x\right\} .
$$

By Theorem25, we have the map

$$
p_{\widehat{\delta}}: \mathbb{X}_{d, \widehat{\delta}} \rightarrow Z_{d, \widehat{\delta}}, x \mapsto \lim _{t \rightarrow 0} \delta(t) \cdot x
$$

We recall the following result from $[\mathrm{Ki}, \S 13]$.

Proposition 26. Let $\mathbb{X}$ and $\mathbb{L}$ be as above. Assume further that $\mathbb{X}$ is smooth. Then, we have the following:

(a) $Z_{d, \widehat{\delta}}$ is an open $S^{\delta}$-stable subset of $\mathbb{X}^{\delta}$, where $S^{\delta}$ is the centralizer of $\delta$ in $S$.

(b) $\mathbb{X}_{d, \widehat{\delta}}=\left\{x \in \mathbb{X}: \lim _{t \rightarrow 0} \delta(t) \cdot x \in Z_{d, \widehat{\delta}}\right\}$, and it is stable under $P(\delta)$.

(c) There is a bijective morphism

$$
S \times{ }^{P(\delta)} \mathbb{X}_{d, \widehat{\delta}} \rightarrow \mathbb{X}_{d,\langle\widehat{\delta}\rangle},
$$

which is an isomorphism if $\mathbb{X}_{d,\langle\widehat{\delta}\rangle}$ is normal, where

$$
\mathbb{X}_{d,\langle\widehat{\delta}\rangle}=\bigcup_{g \in S} \mathbb{X}_{d, g \cdot \widehat{\delta}}
$$

Let $\langle M(S)\rangle$ denote the $S$-conjugacy classes in $M(S)$. We have the following result due to Hesselink [He]. 
Proposition 27. For $\mathbb{X}$ and $\mathbb{L}$ as in Proposition 26,

$$
\mathbb{X}=\mathbb{X}^{s} \bigcup \underset{d>0,\langle\widehat{\delta}\rangle \in\langle M(S)\rangle}{\bigcup} \mathbb{X}_{d,\langle\widehat{\delta}\rangle}
$$

is a finite stratification by locally-closed $S$-stable subvarieties of $\mathbb{X}$, where $\mathbb{X}^{s}$ is the set of semistable points of $\mathbb{X}$ with respect to the ample line bundle $\mathbb{L}$.

Proof of Theorem 24. Let $\mathbb{L}$ denote the $G$-linearized line bundle $\mathcal{L}\left(\lambda_{1}\right) \otimes \cdots \otimes \mathcal{L}\left(\lambda_{s}\right)$ on $(G / B)^{s}$ and let $P_{1}, \ldots, P_{s}$ be the standard parabolic subgroups such that $\mathbb{L}$ descends as an ample line bundle $\overline{\mathbb{L}}$ on $\mathbb{X}(\lambda):=G / P_{1} \times \cdots \times G / P_{s}$. As earlier, we call a point $x \in(G / B)^{s}$ semistable (with respect to, not necessarily ample, $\mathbb{L}$ ) if its image in $\mathbb{X}(\lambda)$ under the canonical map $\pi:(G / B)^{s} \rightarrow \mathbb{X}(\lambda)$ is semistable. Since the map $\pi$ induces an isomorphism of $G$-modules:

$$
H^{0}\left(\mathbb{X}(\lambda), \overline{\mathbb{L}}^{N}\right) \simeq H^{0}\left((G / B)^{s}, \mathbb{L}^{N}\right), \forall N>0
$$

the condition (a) of Theorem 24 is equivalent to the following condition:

(c) The set of semistable points of $(G / B)^{s}$ with respect to $\mathbb{L}$ is nonempty.

The implication $(\mathrm{a}) \Rightarrow(\mathrm{b})$ of Theorem 24 is of course a special case of Theorem 10.

To prove the implication $(\mathrm{b}) \Rightarrow(\mathrm{a})$ in Theorem 24 , we need to recall the following result due to Kapovich-Leeb-Millson $\left[\mathrm{KLM}_{1}\right]$. (For a self-contained algebrogeometric proof of this result, see $\left[\mathrm{BK}_{1}, \S 7.4\right]$.) Suppose that $x=\left(\bar{g}_{1}, \ldots, \bar{g}_{s}\right) \in$ $(G / B)^{s}$ is an unstable point and $P(x)$ the Kempf's parabolic associated to $\pi(x)$. Let $\widehat{\delta}=[\delta, a]$ be a Kempf's OPS associated to $\pi(x)$. Express $\delta(t)=f \gamma(t) f^{-1}$, where $\dot{\gamma} \in \mathfrak{h}_{+}$. Then, the Kempf's parabolic $P(\gamma)$ is a standard parabolic. Define $w_{j} \in W / W_{P(\gamma)}$ by $f P(\gamma) \in g_{j} B w_{j} P(\gamma)$ for $j=1, \ldots, s$. Let $P$ be a maximal parabolic containing $P(\gamma)$.

\section{Theorem 28.}

(i) The intersection $\bigcap_{j=1}^{s} g_{j} B w_{j} P \subset G / P$ is the singleton $\{f P\}$.

(ii) For the simple root $\alpha_{i_{P}} \in \Delta \backslash \Delta(P), \sum_{j=1}^{s} \lambda_{j}\left(w_{j} x_{i_{P}}\right)>0$.

Now, we come to the proof of the implication $(b) \Rightarrow(a)$ in Theorem 24. Assume, if possible, that (a) (equivalently (c) as above) is false, i.e., the set of semistable points of $(G / B)^{s}$ is empty. Thus, any point $x=\left(\bar{g}_{1}, \ldots, \bar{g}_{s}\right) \in(G / B)^{s}$ is unstable. Choose a general $x$ so that for each standard parabolic $\widetilde{P}$ in $G$ and any $\left(z_{1}, \ldots, z_{s}\right) \in W^{s}$, the intersection $g_{1} B z_{1} \widetilde{P} \cap \cdots \cap g_{s} B z_{s} \widetilde{P}$ is transverse (possibly empty) and dense in $g_{1} \overline{B z_{1} \widetilde{P}} \cap \cdots \cap g_{s} \overline{B z_{s} \widetilde{P}}$ (cf. Theorem 6 ). Let $\widehat{\delta}=$ $[\delta, a], P, \gamma, f, w_{j}$ be as above associated to $x$. It follows from Theorem 28 that $\bigcap_{j=1}^{s} g_{j} B w_{j} P \subset G / P$ is the single point $f$ and, since $x$ is general, we get

$$
\left[X_{w_{1}}^{P}\right] \ldots\left[X_{w_{s}}^{P}\right]=\left[X_{e}^{P}\right] \in H^{*}(G / P, \mathbb{Z})
$$

We now claim that the $s$-tuple $\left(w_{1}, \ldots, w_{s}\right) \in\left(W / W_{P}\right)^{s}$ is $L$-movable. 


\section{SHRAWAN KUMAR}

Write $g_{j}=f p_{j} w_{j}^{-1} b_{j}$, for some $p_{j} \in P(\gamma)$ and $b_{j} \in B$ (where we have abused the notation to also denote a lift of $w_{j}$ in $N(H)$ by $\left.w_{j}\right)$. Hence,

$$
\delta(t) \bar{g}_{j}=f \gamma(t) p_{j} w_{j}^{-1} B=f \gamma(t) p_{j} \gamma^{-1}(t) w_{j}^{-1} B \in G / B .
$$

Define, $l_{j}=\lim _{t \rightarrow 0} \gamma(t) p_{j} \gamma^{-1}(t)$. Then, $l_{j} \in L(\gamma)$. Therefore,

$$
\lim _{t \rightarrow 0} \delta(t) x=\left(f l_{1} w_{1}^{-1} B, \ldots, f l_{s} w_{s}^{-1} B\right) .
$$

By Theorem 25, $\widehat{\delta} \in \Lambda\left(\pi\left(\lim _{t \rightarrow 0} \delta(t) x\right)\right)$. We further note that clearly

$$
f P(\gamma) \in \cap_{j}\left(f l_{j} w_{j}^{-1}\right) B w_{j} P(\gamma) .
$$

Applying Theorem 28 to the unstable point $x_{o}=\lim _{t \rightarrow 0} \delta(t) x$ yields: $f P$ is the only point in the intersection $\bigcap_{j=1}^{s} f l_{j} w_{j}^{-1} B w_{j} P$, i.e., translating by $f$, we get: $\dot{e}=e P$ is the only point in the intersection $\Omega:=\bigcap_{j=1}^{s} l_{j} w_{j}^{-1} B w_{j} P$. Thus, $\operatorname{dim} \Omega=$ 0 . By (43), the expected dimension of $\Omega$ is 0 as well. Now, the variety $l_{j} w_{j}^{-1} B w_{j} P$ is isomorphic with the closed subvariety $\left(l_{j} w_{j}^{-1} B w_{j} l_{j}^{-1}\right) \cap U_{P}^{-}$of $U_{P}^{-}$, where $U_{P}^{-}$ is the opposite unipotent radical of $P$. But, the variety $\left(l_{j} w_{j}^{-1} B w_{j} l_{j}^{-1}\right) \cap U_{P}^{-}$is isomorphic with a linear subspace of $\mathfrak{u}_{P}^{-}$via the exponential map. Thus, since $\operatorname{dim} \Omega$ and the expected dimension of $\Omega$ are both 0 , we get that the intersection $\bigcap l_{j} w_{j}^{-1} B w_{j} P$ is transverse at $e \in G / P$, proving that $\left(w_{1}, \ldots, w_{s}\right)$ is $L$-movable. Now, part (ii) of Theorem 28 contradicts the inequality $I_{\left(w_{1}, \ldots, w_{s}\right)}^{P}$. Thus, the set of semistable points of $(G / B)^{s}$ is nonempty, proving condition (a) of Theorem 24 .

The following result follows easily by combining Theorems 24 and 5 . For a maximal parabolic $P$, let $\alpha_{i_{P}}$ be the unique simple root not in the Levi of $P$ and we set $\omega_{P}:=\omega_{i_{P}}$.

Corollary 29. Let $\left(h_{1}, \ldots, h_{s}\right) \in \mathfrak{h}_{+}^{s}$. Then the following are equivalent:

(a) $\left(h_{1}, \ldots, h_{s}\right) \in \bar{\Gamma}_{s}(\mathfrak{g})$.

(b) For every standard maximal parabolic subgroup $P$ in $G$ and every choice of s-tuples $\left(w_{1}, \ldots, w_{s}\right) \in\left(W^{P}\right)^{s}$ such that

$$
\left[X_{w_{1}}^{P}\right] \odot_{0} \cdots \odot_{0}\left[X_{w_{s}}^{P}\right]=\left[X_{e}^{P}\right],
$$

the following inequality holds:

$$
\omega_{P}\left(\sum_{j=1}^{s} w_{j}^{-1} h_{j}\right) \leq 0 .
$$

Remark 5. The cone $\bar{\Gamma}_{3}(\mathfrak{g}) \subset \mathfrak{h}_{+}^{3}$ is quite explicitly determined for any simple $\mathfrak{g}$ of rank 2 in $\left[\mathrm{KLM}_{1}, \S 7\right]$; any simple $\mathfrak{g}$ of rank 3 in $[\mathrm{KuLM}]$ (reproduced in Section $14)$; and for $\mathfrak{g}=s o(8)$ in $[\mathrm{KKM}]$. It has $12(6+6) ; 18(9+9) ; 30(15+15) ; 41(10+21+$ 10); $93(18+48+27) ; 93(18+48+27) ; 294(36+186+36+36) ; 1290(126+519+519+$ 126); $26661(348+1662+4857+14589+4857+348)$ facets inside $\mathfrak{h}_{+}^{3}$ (intersecting the interior of $\mathfrak{h}_{+}^{3}$ ) for $\mathfrak{g}$ of type $A_{2} ; B_{2} ; G_{2} ; A_{3} ; B_{3} ; C_{3} ; D_{4} ; F_{4} ; E_{6}$ respectively. The notation $30(15+15)$ means that there are 15 (irredundant) inequalities coming from $G / P_{1}$ and there are 15 inequalities coming from $G / P_{2}$ via Corollary 29(b). (The indexing convention is as in [Bo, Planche I-IX].) 


\section{Study of the saturated restriction semigroup and irredundancy of its inequalities}

This section is based on the work of Ressayre $\left[\mathrm{R}_{1}\right]$ (also see $[\mathrm{Br}]$ ).

Let $G \subset \widehat{G}$ be connected reductive complex algebraic groups. We fix a maximal torus $H$ (resp. $\widehat{H}$ ) and a Borel subgroup $H \subset B$ (resp. $\widehat{H} \subset \widehat{B}$ ) of $G$ (resp. $\widehat{G}$ ) such that $H \subset \widehat{H}$ and $B \subset \widehat{B}$. We shall follow the notation from Section 2 for $G$ and the corresponding objects for $\widehat{G}$ will be denoted by a hat on the top.

Define the saturated restriction semigroup

$$
\Gamma(G, \widehat{G})=\left\{(\lambda, \widehat{\lambda}) \in \Lambda_{+} \times \widehat{\Lambda}_{+}:[V(N \lambda) \otimes \widehat{V}(N \widehat{\lambda})]^{G} \neq 0, \text { for some } N \geq 1\right\} .
$$

The aim of this section is to determine this semigroup in terms of an irredundant system of inequalities.

Lemma 30. The interior of $\Gamma(G, \widehat{G})_{\mathbb{R}}$ inside $\Lambda_{+}(\mathbb{R}) \times \widehat{\Lambda}_{+}(\mathbb{R})$ is nonempty if and only if no nonzero ideal of $\mathfrak{g}$ is an ideal of $\widehat{\mathfrak{g}}$, where $\Gamma(G, \widehat{G})_{\mathbb{R}}$ is the cone inside $\Lambda_{+}(\mathbb{R}) \times \widehat{\Lambda}_{+}(\mathbb{R})$ generated by $\Gamma(G, \widehat{G})$ and $\Lambda_{+}(\mathbb{R})$ is the cone inside $\Lambda \otimes_{\mathbb{Z}} \mathbb{R}$ generated by $\Lambda_{+}$and $\widehat{\Lambda}_{+}(\mathbb{R})$ has a similar meaning.

Proof. By $\left[\mathrm{MR}\right.$, Cor. 1], the codimension of $\Gamma(G, \widehat{G})_{\mathbb{R}}$ in $\Lambda_{+}(\mathbb{R}) \times \widehat{\Lambda}_{+}(\mathbb{R})$ is the dimension of the kernel $H_{o}$ of the adjoint action Ad $: H \rightarrow \operatorname{Aut}(\widehat{G} / G)$. Clearly, $H_{o}=\bigcap_{\widehat{g} \in \widehat{G}} \widehat{g} G \widehat{g}^{-1}$, which is a normal subgroup of $\widehat{G}$ contained in $G$. Moreover, any normal subgroup $N$ of $G$ which is also normal in $\widehat{G}$ is of course contained in $H_{o}$. This proves the lemma.

Remark 6. A stronger result than the above lemma is proved in [PR, Thm. 4].

For any $G$-dominant OPS $\delta \in O(H)$, (i.e., $\dot{\delta} \in \mathfrak{h}_{+}$), let $P(\delta)$ (resp. $\widehat{P}(\delta)$ ) be the Kempf's parabolic associated to $\delta$ inside $G$ (resp. $\widehat{G}$ ), cf. the identity (11). Since $\delta$ is dominant for $G, P(\delta)$ is a standard parabolic subgroup of $G$.

Analogous to the Definition 4, we define the following.

Definition 8. Let $(w, \widehat{w}) \in W^{P(\delta)} \times \widehat{W}^{\widehat{P}(\delta)}$ be such that

$$
\ell(\widehat{w})+\ell(w)=\operatorname{dim} \widehat{G} / \widehat{P}(\delta) .
$$

Then, we say that $(w, \widehat{w})$ is L-movable if the canonical map

$$
\left.T_{e}\left(\Phi_{w}^{P(\delta)}\right) \stackrel{(d \iota)_{e}}{\longrightarrow} \frac{T_{\widehat{e}}(\widehat{G} / \widehat{P}(\delta))}{T_{\widehat{e}} \widehat{l} \widehat{l} \widehat{\Phi}_{\widehat{w}}(\delta)}\right)
$$

is an isomorphism for some $\widehat{l} \in \widehat{L}(\delta)$, where $e$ (resp. $\widehat{e})$ is the base point $1 \cdot P(\delta) \in$ $G / P(\delta)($ resp. $1 \cdot \widehat{P}(\delta) \in \widehat{G} / \widehat{P}(\delta))$.

For any $w \in W / W_{P(\delta)}$, let $\gamma_{w}^{P(\delta)}$ be the sum of the $H$-weights in the normal space $T_{e}(G / P(\delta)) / T_{e}\left(\Phi_{w}^{P(\delta)}\right)$. We similarly define $\widehat{\gamma}_{\widehat{w}}^{\widehat{P}(\delta)}$ for any $\widehat{w} \in \widehat{W} / \widehat{W}_{\widehat{P}(\delta)}$. Then, it is easy to see from Lemma 19 (since $\delta$ is $G$-dominant) that

$$
\gamma_{w}^{P(\delta)}(\dot{\delta})=-\left(\rho+w^{-1} \rho\right)(\dot{\delta}) .
$$


Moreover,

$$
\widehat{\gamma}_{\widehat{w}}^{\widehat{P}(\delta)}(\dot{\delta})=-\left(\widehat{v}^{-1} \widehat{\rho}+\widehat{w}^{-1} \widehat{\rho}\right)(\dot{\delta})
$$

where $\widehat{v} \in \widehat{W}$ is such that $\widehat{v}(\dot{\delta}) \in \widehat{\mathfrak{h}}_{+}$.

We have the following result analogous to Proposition 21.

Proposition 31. Let $(w, \widehat{w}) \in W^{P(\delta)} \times \widehat{W}^{\widehat{P}(\delta)}$ be such that

$$
\ell(\widehat{w})+\ell(w)=\operatorname{dim} \widehat{G} / \widehat{P}(\delta)
$$

Then, the following are equivalent:

(a) $(w, \widehat{w})$ is L-movable for the embedding $\iota: G / P(\delta) \rightarrow \widehat{G} / \widehat{P}(\delta)$,

(b) $\left[X_{w}^{P(\delta)}\right] \cdot \iota^{*}\left[\widehat{X}_{\widehat{w}}^{\widehat{P}^{(\delta)}}\right]=d[\mathrm{pt}]$, for some $d \neq 0$, and

$$
\widehat{\gamma}_{\widehat{w}}(\delta)(\dot{\delta})=\gamma_{e}^{P(\delta)}(\dot{\delta})-\gamma_{w}^{P(\delta)}(\dot{\delta})
$$

Proof. Let $T$ (resp. $\widehat{T}$ ) be the tangent space of $G / P(\delta)$ (resp. $\widehat{G} / \widehat{P}(\delta)$ ) at the base point $1 \cdot P(\delta)$ (resp. $1 \cdot \widehat{P}(\delta)$ ). Similarly, let $T_{w}$ (resp. $\widehat{T}_{\widehat{w}}$ ) be the tangent space of $\Phi_{w}^{P(\delta)}$ (resp. $\widehat{\Phi}_{\widehat{w}}^{\widehat{P}(\delta)}$ ) at the base point. Then, $\widehat{T}$ and $\widehat{T}_{\widehat{w}}$ are $\widehat{B}_{\widehat{L}(\delta)}$ modules since $\widehat{B}_{\widehat{L}(\delta)}$ stabilizes $\widehat{\Phi}_{\widehat{w}}^{\widehat{P}(\delta)}$ keeping the base point $1 \cdot \widehat{P}(\delta)$ fixed, where $\widehat{B}_{\widehat{L}(\delta)}$ is the Borel subgroup $\widehat{B} \cap \widehat{L}(\delta)$ of $\widehat{L}(\delta)$.

Let $\widehat{\mathcal{T}}$ (resp. $\left.\widehat{\mathcal{T}} / \widehat{\mathcal{T}}_{\widehat{w}}\right)$ be the vector bundle $\widehat{P}(\delta) \times{ }^{\widehat{B}} \widehat{L}(\delta) \widehat{T}\left(\operatorname{resp} . \widehat{P}(\delta) \times{ }^{\widehat{B}} \widehat{L}(\delta)\left(\widehat{T} / \widehat{T}_{\widehat{w}}\right)\right.$ ) over the base space $\widehat{P}(\delta) / \widehat{B}_{\widehat{L}(\delta)}$. For any vector space $V$, we let $\epsilon(V)$ be the trivial vector bundle $\widehat{P}(\delta) / \widehat{B}_{\widehat{L}(\delta)} \times V$ over $\widehat{P}(\delta) / \widehat{B}_{\widehat{L}(\delta)}$.

We have the $B_{L(\delta)}$-equivariant bundle map

$$
\Theta: \epsilon\left(T_{w}\right) \rightarrow \widehat{\mathcal{T}} / \widehat{\mathcal{T}}_{\widehat{w}}
$$

obtained as the composition

$$
\epsilon\left(T_{w}\right) \hookrightarrow \epsilon(\widehat{T}) \stackrel{\alpha}{\rightarrow} \widehat{\mathcal{T}} \rightarrow \widehat{\mathcal{T}} / \widehat{\mathcal{T}}_{\widehat{w}},
$$

where $B_{L(\delta)}:=B \cap L(\delta), B_{L(\delta)}$ acts on $\epsilon\left(T_{w}\right)$ diagonally, the first map is the canonical inclusion, the last map is induced by the projection and the $\widehat{P}(\delta)$-equivariant isomorphism $\alpha$ is given by

$$
\alpha\left(p \widehat{B}_{\widehat{L}(\delta)}, v\right)=\left[\widehat{p}, \widehat{p}^{-1} v\right], \quad \text { for } \widehat{p} \in \widehat{P}(\delta), v \in \widehat{T} .
$$

(Observe that $\widehat{T}$ is canonically a $\widehat{P}(\delta)$-module.)

By assumption, the map $\Theta$ is a bundle map between the bundles of the same rank. Hence, $\Theta$ induces a bundle map $\theta$ by taking the top exterior powers

$$
\theta: \epsilon\left(\operatorname{det} T_{w}\right) \rightarrow \operatorname{det}\left(\widehat{\mathcal{T}} / \widehat{\mathcal{T}}_{\widehat{w}}\right)
$$


which can be viewed as a $B_{L(\delta)}$-invariant section in

$$
H^{0}\left(\widehat{P}(\delta) / \widehat{B}_{\widehat{L}(\delta)},\left(\epsilon\left(\operatorname{det} T_{w}\right)^{-1}\right) \otimes \operatorname{det}\left(\widehat{\mathcal{T}} / \widehat{\mathcal{T}}_{\widehat{w}}\right)\right)
$$

By definition, $(w, \widehat{w})$ is $L$-movable if and only if the section $\theta_{\mid\left(\widehat{L}(\delta) / \widehat{B}_{\widehat{L}(\delta)}\right)} \neq 0$. Now, the rest of the proof of this proposition is identical to the proof of Proposition 21 and Lemma 20, since the image of $\delta$ is central in $\widehat{L}(\delta)$. (Since $\operatorname{Im} \delta$ is central in $\widehat{L}(\delta)$, it is easy to see, by the same proof as that of Lemma 9 , that

$$
\left.\mu^{\left(\epsilon\left(\operatorname{det} T_{w}\right)^{-1}\right) \otimes \operatorname{det}\left(\widehat{\mathcal{T}} / \widehat{\mathcal{T}}_{\widehat{w}}\right)}(\widehat{p}, \delta)=\widehat{\gamma}_{\widehat{w}}^{\widehat{P}(\delta)}(\dot{\delta})-\gamma_{e}^{P(\delta)}(\dot{\delta})+\gamma_{w}^{P(\delta)}(\dot{\delta}) .\right)
$$

This proves the proposition.

For any $\delta \in 0(H)$, the centralizer of $\delta$ in $G$ :

$$
G^{\delta}:=\left\{g \in G: g \delta(t)=\delta(t) g \text { for all } t \in \mathbb{G}_{m}\right\}
$$

is the Levi subgroup $L(\delta)$ (containing $H$ ) of the Kempf's parabolic subgroup $P(\delta)$. Let $Y$ be a smooth projective $G$-variety. Let $C$ be an irreducible component of $Y^{\delta}$ and let

$$
C_{+}=\left\{y \in Y: \lim _{t \rightarrow 0} \delta(t) \cdot y \text { lies in } C\right\} .
$$

Then, $C$ is a closed smooth $L(\delta)$-stable subvariety of $Y$ (since $L(\delta)$ is connected); $C_{+}$is a $P(\delta)$-stable, smooth, irreducible, locally-closed subvariety of $Y$ (by a result of Bialynicki-Birula); and the map $\pi_{\delta}: C_{+} \rightarrow C, y \mapsto \lim _{t \rightarrow 0} \delta(t) \cdot y$ is a morphism.

Consider the $G$-equivariant morphism

$$
\eta: G \times{ }^{P(\delta)} C_{+} \rightarrow Y, \quad[g, y] \mapsto g \cdot y
$$

The following definition is due to Ressayre $\left[\mathrm{R}_{1}\right]$.

Definition 9. The pair $(C, \delta)$ is called a well-covering pair if there exists a $P(\delta)$ stable open (irreducible) subset $C_{+}^{o}$ of $C_{+}$such that $C_{+}^{o} \cap C$ is nonempty and the map $\eta_{o}=\eta_{\mid\left(G \times{ }^{P(\delta)} C_{+}^{o}\right)}$ is an isomorphism onto an open subset of $Y$.

Now, we take $Y=X:=G / B \times \widehat{G} / \widehat{B}$ with the diagonal action of $G$ and let $\delta \in O(H)$ be a $G$-dominant OPS. It is easy to see that

$$
X^{\delta}=\bigsqcup C_{\delta}(w, \widehat{w})
$$

where

$$
C_{\delta}(w, \widehat{w}):=\left(L(\delta) \cdot w^{-1} B / B\right) \times\left(\widehat{L}(\delta) \cdot \widehat{w}^{-1} \widehat{B} / \widehat{B}\right),
$$

and the union runs over $(w, \widehat{w}) \in W^{P(\delta)} \times \widehat{W}^{\widehat{P}(\delta)}$. Further, it is easy to see that

$$
C_{\delta}(w, \widehat{w})_{+}=\left(P(\delta) \cdot w^{-1} B / B\right) \times\left(\widehat{P}(\delta) \cdot \widehat{w}^{-1} \widehat{B} / \widehat{B}\right)
$$




\section{SHRAWAN KUMAR}

Lemma 32. For any $(w, \widehat{w}) \in W^{P(\delta)} \times \widehat{W}^{\widehat{P}(\delta)}$, the following are equivalent:

(a) The pair $\left(C_{\delta}(w, \widehat{w}), \delta\right)$ is a well-covering pair.

(b) The pair $(w, \widehat{w})$ is L-movable for the embedding $\iota: G / P(\delta) \hookrightarrow \widehat{G} / \widehat{P}(\delta)$ and

$$
\left[X_{w}^{P(\delta)}\right] \cdot \iota^{*}\left[\widehat{X}_{\widehat{w}}^{\widehat{P}(\delta)}\right]=[\mathrm{pt}] .
$$

Proof. The projection $\pi: G \times{ }^{P(\delta)} C_{\delta}(w, \widehat{w})_{+} \rightarrow G / P(\delta)$ induces an isomorphism between the fiber $\eta^{-1}((g B, \widehat{g} \widehat{B}))$ and the locally closed subscheme $\left(g C_{w}^{P(\delta)}\right) \cap$ $\left(\widehat{g} \widehat{C}_{\widehat{w}}(\delta)\right.$ of $\widehat{G} / \widehat{P}(\delta)$, for any $g \in G$ and $\widehat{g} \in \widehat{G}$.

Proof of $(\mathrm{a}) \Rightarrow(\mathrm{b})$ : Since $\left(C_{\delta}(w, \widehat{w}), \delta\right)$ is a well-covering pair, there exist $l \in$ $L(\delta)$ and $\widehat{l} \in \widehat{L}(\delta)$ such that $\eta_{o}^{-1}\left(l w^{-1} B, \widehat{l} \widehat{w}^{-1} \widehat{B}\right)$ is a reduced one point. Thus, $\eta^{-1}\left(l w^{-1} B, \widehat{l}^{-1} \widehat{B}\right) \simeq\left(l \Phi_{w}^{P(\delta)}\right) \cap\left(\widehat{l}_{\widehat{w}}^{\widehat{P}(\delta)}\right)$ is a reduced single point in a neighborhood of $1 \cdot \widehat{P}(\delta)$, showing that $(w, \widehat{w})$ is $L$-movable.

Take any (general) $y_{o}=\left(g_{o} B, \widehat{g}_{o} \widehat{B}\right) \in \operatorname{Im}\left(\eta_{o}\right)$ so that $\eta_{o}^{-1}\left(y_{o}\right)=\eta^{-1}\left(y_{o}\right)$ and the intersection $\left(g_{o} C_{w}^{P(\delta)}\right) \cap\left(\widehat{g}_{o} \widehat{C}_{\widehat{w}}{ }^{(\delta)}\right)$ is proper inside $\widehat{G} / \widehat{P}(\delta)$ and dense in $\left(g_{o} X_{w}^{P(\delta)}\right) \cap$ $\left(\widehat{g}_{o} \widehat{X}_{\widehat{w}} \widehat{P}^{(\delta)}\right)$. Such a $y_{o}$ exists since $\operatorname{Im}\left(\eta_{o}\right)$ is open in $X$. Now, $\eta_{o}^{-1}\left(y_{o}\right)=\eta^{-1}\left(y_{o}\right)$ is a single reduced point by the assumption. Thus, $\left(g_{o} C_{w}^{P(\delta)}\right) \cap\left(\widehat{g}_{o} \widehat{C}_{\widehat{w}}(\delta)\right)$ is a single reduced point, showing that

$$
\left[X_{w}^{P(\delta)}\right] \cdot \iota^{*}\left[\widehat{X}_{\widehat{w}}^{\widehat{P}(\delta)}\right]=[\mathrm{pt}] .
$$

Proof of (b) $\Rightarrow(\mathrm{a})$ : Take a $G$-stable open subset $V \subset G / B \times \widehat{G} / \widehat{B}$ so that for any $(g B, \widehat{g} \widehat{B}) \in V$, the intersection $g C_{w}^{P(\delta)} \cap\left(\widehat{g} \widehat{C}_{\widehat{w}} \widehat{P}^{(\delta)}\right)$ is transverse inside $\widehat{G} / \widehat{P}(\delta)$ and dense in $\left(g X_{w}^{P(\delta)}\right) \cap\left(\widehat{g} \widehat{X}_{\widehat{w}}^{\widehat{P}(\delta)}\right)$. Since $\left[X_{w}^{P(\delta)}\right] \cdot \iota^{*}\left[\widehat{X}_{\widehat{w}}^{\widehat{P}}(\delta)\right]=[\mathrm{pt}]$ by assumption, for any $(g B, \widehat{g} \widehat{B}) \in V$, the scheme $\left(g C_{w}^{P(\delta)}\right) \cap\left(\widehat{g} \widehat{C}_{\widehat{w}}^{\widehat{P}(\delta)}\right)$ is a reduced single point. Thus, $\eta_{\mid \eta^{-1}(V)}: \eta^{-1}(V) \rightarrow V$ is an isomorphism; in particular, $\eta: G \times{ }^{P(\delta)} C_{\delta}(w, \widehat{w})_{+} \rightarrow$ $X$ is a birational map. Let $V^{\prime}$ be the open subset

$$
V^{\prime}:=\left\{y \in G \times{ }^{P(\delta)} C_{\delta}(w, \widehat{w})_{+}:(d \eta)_{y} \text { is an isomorphism }\right\} .
$$

Then, $V^{\prime}$ is clearly $G$-stable and hence can be written as $G \times{ }^{P(\delta)} C_{+}^{o}$, for a $P(\delta)$ stable open subset $C_{+}^{o}$ of $C_{\delta}(w, \widehat{w})_{+}$. Since $\eta_{\mid v^{\prime}}$ is a smooth birational morphism, it is an isomorphism onto an open subset of $X$ (cf. [Sh, Cor. $1, \S 4.3$, Chap. II]). Since $(w, \widehat{w})$ is $L$-movable, the point $1 \cdot \widehat{P}(\delta)$ is a reduced isolated point of the scheme $\left(l \Phi_{w}^{P(\delta)}\right) \cap\left(\widehat{l} \widehat{\Phi}_{\widehat{w}}^{\widehat{P}}(\delta)\right)$ for some $l \in L(\delta)$ and $\widehat{l} \in \widehat{L}(\delta)$. Hence $\left[1,\left(l w^{-1} B, \widehat{l} \widehat{w}^{-1} \widehat{B}\right)\right] \in V^{\prime}$. Thus, $\left(C_{\delta}(w, \widehat{w}), \delta\right)$ is a well-covering pair.

Definition 10. We will call a nonzero $G$-dominant indivisible OPS $\delta \in O(H)$ special for the pair $(G, \widehat{G})$ if $\mathbb{C} \dot{\delta}=\cap \operatorname{Ker} \beta$, where the intersection runs over the set of $\mathfrak{h}$-weights of $\widehat{\mathfrak{l}}(\delta) / \mathfrak{l}(\delta)$, where $\mathfrak{l}(\delta)$ (resp. $\widehat{\mathfrak{l}}(\delta)$ ) denotes the Lie algebra of $L(\delta)$ (resp. $\widehat{L}(\delta)$ ).

We denote the set of all special OPS for the pair $(G, \widehat{G})$ by $\mathfrak{S}=\mathfrak{S}(G, \widehat{G})$. Clearly, it is a finite set. Let us enumerate

$$
\mathfrak{S}(G, \widehat{G})=\left\{\delta_{1}, \ldots, \delta_{q}\right\}
$$




\section{EIGENVALUE PROBLEM}

Theorem 33. With the notation as above, assume that no nonzero ideal of $\mathfrak{g}$ is an ideal of $\widehat{\mathfrak{g}}$. Let $(\lambda, \widehat{\lambda}) \in \Lambda_{+} \times \widehat{\Lambda}_{+}$. Then, the following three conditions are equivalent:

(a) $(\lambda, \widehat{\lambda}) \in \Gamma(G, \widehat{G})$.

(b) For any $G$-dominant $\delta \in O(H)$, and any $(w, \widehat{w}) \in W^{P(\delta)} \times \widehat{W^{P}(\delta)}$ such that $\left[X_{w}^{P(\delta)}\right] \cdot \iota^{*}\left(\left[\widehat{X}_{\widehat{w}}^{\widehat{P}(\delta)}\right]\right) \neq 0$ in $H^{*}(G / P(\delta), \mathbb{Z})$, where $\widehat{X}_{\widehat{w}}^{\widehat{P}(\delta)}:=\overline{\widehat{B} \widehat{w} \widehat{P}(\delta) / \widehat{P}(\delta)} \subset$ $\widehat{G} / \widehat{P}(\delta)$ (even though $\widehat{P}(\delta)$ may not be a standard parabolic subgroup) and $\iota: G / P(\delta) \rightarrow \widehat{G} / \widehat{P}(\delta)$ is the canonical embedding, we have

$$
I_{(w, \widehat{w})}^{\delta}: \quad \lambda(w \dot{\delta})+\widehat{\lambda}(\widehat{w} \dot{\delta}) \leq 0
$$

(c) For any $O P S \delta_{i} \in \mathfrak{S}(G, \widehat{G})$ and any $(w, \widehat{w}) \in W^{P\left(\delta_{i}\right)} \times \widehat{W^{P}\left(\delta_{i}\right)}$ such that

$\left(c_{1}\right)\left[X_{w}^{P\left(\delta_{i}\right)}\right] \cdot \iota^{*}\left(\left[\widehat{X}_{\widehat{w}}^{\widehat{P}^{(}\left(\delta_{i}\right)}\right]\right)=\left[X_{e}^{P\left(\delta_{i}\right)}\right] \in H^{*}\left(G / P\left(\delta_{i}\right), \mathbb{Z}\right)$, and

$\left(c_{2}\right) \gamma_{e}^{P\left(\delta_{i}\right)}\left(\dot{\delta}_{i}\right)-\gamma_{w}^{P\left(\delta_{i}\right)}\left(\dot{\delta}_{i}\right)=\widehat{\gamma}_{\widehat{w}}\left(\delta_{i}\right)\left(\dot{\delta}_{i}\right)$,

the inequality $I_{(w, \widehat{w})}^{\delta_{i}}($ as in $(50))$ is satisfied.

Proof. For a dominant pair $(\lambda, \widehat{\lambda}) \in \Lambda_{+} \times \widehat{\Lambda}_{+}$, we have the line bundle

$$
\mathcal{L}(\lambda \otimes \widehat{\lambda}):=\mathcal{L}(\lambda) \otimes \mathcal{L}(\widehat{\lambda})
$$

on $X=G / B \times \widehat{G} / \widehat{B}$. Let $P(\lambda), \widehat{P}(\widehat{\lambda})$ be the unique standard parabolic subgroups such that the line bundle $\mathcal{L}(\lambda \otimes \widehat{\lambda})$ descends as an ample line bundle $\mathbb{L}(\lambda \otimes \hat{\lambda})$ on $X(\lambda, \widehat{\lambda}):=G / P(\lambda) \times \widehat{G} / \widehat{P}(\widehat{\lambda})$. As earlier, we call a point $(g B, \widehat{g} \widehat{B}) \in X$ semistable with respect to the line bundle $\mathcal{L}(\lambda \otimes \widehat{\lambda})$ if $\pi(g B, \widehat{g} \widehat{B})$ is $G$-semistable with respect to the ample line bundle $\mathbb{L}(\lambda \otimes \widehat{\lambda})$, where $\pi: X \rightarrow X(\lambda, \hat{\lambda})$ is the canonical projection.

Proof of $(\mathrm{a}) \Rightarrow(\mathrm{b})$ : We abbreviate $P(\delta)$ (resp. $\widehat{P}(\delta)$ ) by $P$ (resp. $\widehat{P}$ ). Pick any (general) $(g, \widehat{g}) \in G \times \widehat{G}$ satisfying the following:

$$
\begin{aligned}
& \widehat{g} \widehat{C}_{\widehat{w}}^{\widehat{P}} \cap g C_{w}^{P} \text { and } \widehat{g} \widehat{X}_{\widehat{w}}^{\widehat{P}} \cap g X_{w}^{P} \text { are proper intersections in } \widehat{G} / \widehat{P} \\
& \text { with } \widehat{g} \widehat{C}_{\widehat{w}}^{\widehat{P}} \cap g C_{w}^{P} \text { dense in } \widehat{g} \widehat{X} \widehat{\widehat{w}} \cap g X_{w}^{P},
\end{aligned}
$$

and

$$
(g B, \widehat{g} \widehat{B}) \text { is a } G \text {-semistable point of } X \text { with respect to } \mathcal{L}(\lambda \otimes \widehat{\lambda}) .
$$

Then, by the assumption on the cohomology product as in (b), we get $h \in G$ such that $h^{-1} P \in \widehat{g} \widehat{C}_{\widehat{w}} \cap g C_{w}^{P}$. Pick $\widehat{v} \in \widehat{W}$ such that $\widehat{v} \dot{\delta} \in \widehat{\mathfrak{h}}_{+}$. Then,

$$
\begin{aligned}
\mu^{\mathcal{L}(\lambda \otimes \widehat{\lambda})}((h g B, h \widehat{g} \widehat{B}), \delta) & =\mu^{\mathcal{L}(\lambda)}(h g B, \delta)+\mu^{\mathcal{L}(\widehat{\lambda})}(h \widehat{g} \widehat{B}, \delta) \\
& =\mu^{\mathcal{L}(\lambda)}(h g B, \delta)+\mu^{\mathcal{L}(\widehat{\lambda})}(\widehat{v} h \widehat{g} \widehat{B}, \widehat{v} \cdot \delta) \\
& =-\lambda(w \dot{\delta})-\widehat{\lambda}\left(\widehat{w} \widehat{v}^{-1} \widehat{v} \dot{\delta}\right), \text { by Lemma } 9 \\
& \geq 0, \text { by Proposition } 8 .
\end{aligned}
$$


This proves (b).

Proof of (b) $\Rightarrow$ (a): Pick any (general) $(g, \widehat{g}) \in G \times \widehat{G}$ satisfying the equation (51) for any $G$-dominant weight $\delta_{o} \in O(H)$ and any pairs $(w, \widehat{w}) \in W^{P\left(\delta_{o}\right)} \times \widehat{W}^{\widehat{P}\left(\delta_{o}\right)}$. This is possible since there are only finitely many $P\left(\delta_{o}\right)$ and $\widehat{P}\left(\delta_{o}\right)$ as we run through $G$-dominant weights $\delta_{o} \in O(H)$. Fix any $(g, \widehat{g}) \in G \times \widehat{G}$ as above and consider the point $x=(g B, \widehat{g} \widehat{B}) \in X$. If (a) of Theorem 33 were false, then no point of $X$ would be semistable for the line bundle $\mathcal{L}(\lambda \otimes \widehat{\lambda})$. Thus, by Proposition 8 , there exists an OPS $\delta \in O(G)$ (depending upon $x$ ) such that

$$
\mu^{\mathcal{L}(\lambda \otimes \widehat{\lambda})}((g B, \widehat{g} \widehat{B}), \delta)<0 .
$$

Let $\delta=h^{-1} \delta_{o} h$, for $h \in G$ so that $\delta_{o}$ belongs to $O(H)$ and it is $G$-dominant. Pick $w \in W, \widehat{w} \in \widehat{W}$ such that $(h g)^{-1} \in C_{w}^{P\left(\delta_{o}\right)}$ and $(h \widehat{g})^{-1} \in \widehat{C}_{\widehat{w}}^{\widehat{P}\left(\delta_{o}\right)}$. Thus, by Lemma 9 ,

$$
\begin{aligned}
\mu^{\mathcal{L}(\lambda \otimes \widehat{\lambda})}((g B, \widehat{g} \widehat{B}), \delta) & =\mu^{\mathcal{L}(\lambda)}\left(h g B, \delta_{o}\right)+\mu^{\mathcal{L}(\widehat{\lambda})}\left(h \widehat{g} \widehat{B}, \delta_{o}\right) \\
& =-\lambda\left(w \dot{\delta}_{o}\right)-\widehat{\lambda}\left(\widehat{w} \dot{\delta}_{o}\right) \\
& <0, \text { by the inequality }(53) .
\end{aligned}
$$

Now, $\left[X_{w}^{P\left(\delta_{0}\right)}\right] \cdot \iota^{*}\left(\left[\widehat{X}_{\widehat{w}}^{\widehat{P}\left(\delta_{o}\right)}\right]\right) \neq 0$, because of the choice of $(w, \widehat{w})$ and a general point $(g, \widehat{g})$ satisfying the condition (51). This contradicts (b) and hence proves (a).

We now come to the proof of the equivalence of (a) and (c). Since $(a) \Rightarrow(b)$ and clearly $(\mathrm{b}) \Rightarrow(\mathrm{c})$, we get $(\mathrm{a}) \Rightarrow(\mathrm{c})$.

Proof of $(\mathrm{c}) \Rightarrow(\mathrm{a})$ : We first show that for $(\lambda, \widehat{\lambda}) \in \Lambda_{++} \times \widehat{\Lambda}_{++}$, if $(\lambda, \widehat{\lambda}) \notin \Gamma(G, \widehat{G})$, then there exists a well-covering pair $\left(C_{\delta}(w, \widehat{w}), \delta\right)$ (defined by $(48)$ ), for some $G$ dominant $\delta \in O(H)$ and $w \in W^{P(\delta)}, \widehat{w} \in \widehat{W}^{\widehat{P}(\delta)}$, such that the inequality $I_{(w, \widehat{w})}^{\delta}$ is violated, i.e.,

$$
\lambda(w \dot{\delta})+\widehat{\lambda}(\widehat{w} \dot{\delta})>0 .
$$

Since $(\lambda, \widehat{\lambda}) \notin \Gamma(G, \widehat{G})$ (by assumption), the set of $G$-semistable points for the ample line bundle $\mathcal{L}(\lambda \otimes \widehat{\lambda})$ on $X$ is empty. Thus, by Proposition 27, there exists a class $\langle\widehat{\delta}=[\delta, a]\rangle \in\langle M(G)\rangle$ with dominant $\delta$ and a number $d>0$ such that $X_{d,\langle\widehat{\delta}\rangle}$ is a $G$-stable nonempty open subset of $X$; in particular, it is smooth. Hence, by Proposition 26 (c), $X_{d, \widehat{\delta}}$ is irreducible and hence so is $Z_{d, \widehat{\delta}}$ (because of the surjective morphism $p_{\widehat{\delta}}: X_{d, \widehat{\delta}} \rightarrow Z_{d, \widehat{\delta}}$ ). Moreover, by Proposition $26, Z_{d, \widehat{\delta}}$ being an open subset of $X^{\delta}, \bar{Z}_{d, \widehat{\delta}}$ is an irreducible component of $X^{\delta}$. Hence, by the identity (47), there exists $(w, \widehat{w}) \in W^{P(\delta)} \times \widehat{W}^{\widehat{P}(\delta)}$ such that

$$
\bar{Z}_{d, \widehat{\delta}}=C_{\delta}(w, \widehat{w}) .
$$

Since the map $C_{\delta}(w, \widehat{w})_{+} \rightarrow C_{\delta}(w, \widehat{w}), y \mapsto \lim _{t \rightarrow 0} \delta(t) \cdot y$, is a morphism (cf. the discussion before Definition 9), $X_{d, \widehat{\delta}}$ is an open (and $P(\delta)$-stable) subset of $C_{\delta}(w, \widehat{w})_{+}$. 
By Proposition 26(c),

$$
G \times{ }^{P(\delta)} X_{d, \widehat{\delta}} \rightarrow X_{d,\langle\widehat{\delta}\rangle}
$$

is an isomorphism. This shows that $\left(C_{\delta}(w, \widehat{w}), \delta\right)$ is a well-covering pair. By definition, for any $x \in X_{d, \widehat{\delta}}$,

$$
\mu^{\mathcal{L}(\lambda \otimes \widehat{\lambda})}(x, \widehat{\delta}) \leq-1
$$

Thus, by Lemma 9,

$$
-\lambda(w \dot{\delta})-\widehat{\lambda}(\widehat{w} \dot{\delta}) \leq-a
$$

This proves the assertion (54).

Since $\Gamma(G, \widehat{G})_{\mathbb{R}} \subset \Lambda_{+}(\mathbb{R}) \times \widehat{\Lambda}_{+}(\mathbb{R})$ is a convex cone with nonempty interior (by Lemma 30$)$, we get that $\Gamma(G, \widehat{G})_{\mathbb{R}}$ is the cone inside $\Lambda_{+}(\mathbb{R}) \times \widehat{\Lambda}_{+}(\mathbb{R})$ determined by the inequalities

$$
\lambda(w \dot{\delta})+\widehat{\lambda}(\widehat{w} \dot{\delta}) \leq 0
$$

running over all the well-covering pairs $\left(C_{\delta}(w, \widehat{w}), \delta\right)$ with $G$-dominant indecomposable $\delta \in O(H)$. We finally show that, for any well-covering pair $\left(C_{\delta}(w, \widehat{w}), \delta\right)$ with $G$-dominant indecomposable $\delta \in O(H)$, if the hyperplane $F$ :

$$
\lambda(w \dot{\delta})+\widehat{\lambda}(\widehat{w} \dot{\delta})=0
$$

is a (codimension one) facet of $\Gamma(G, \widehat{G})_{\mathbb{R}}$ intersecting $\Lambda_{++}(\mathbb{R}) \times \widehat{\Lambda}_{++}(\mathbb{R})$, then $\delta$ is special.

Let $F_{+}:=F \cap\left(\Lambda_{++}(\mathbb{R}) \times \widehat{\Lambda}_{++}(\mathbb{R})\right)$. For any $(\lambda, \widehat{\lambda}) \in \Lambda_{++} \times \widehat{\Lambda}_{++}$, let $\mathcal{C}(\lambda, \hat{\lambda})$ denote the GIT class of $(\lambda, \widehat{\lambda})$ consisting of those $(\mu, \widehat{\mu}) \in \Lambda_{++} \times \widehat{\Lambda}_{++}$such that the set of $G$-semistable points $X^{s}(\mathcal{L}(\lambda \otimes \hat{\lambda}))=X^{s}(\mathcal{L}(\mu \otimes \widehat{\mu}))$. By [DH] (or [Ro]), $\Lambda_{++} \times \widehat{\Lambda}_{++}$breaks up into finitely many GIT classes, such that the cones generated by them are all locally closed convex cones. Now, for any $(\lambda, \widehat{\lambda}) \in\left(\Lambda_{++} \times \widehat{\Lambda}_{++}\right) \cap$ $\Gamma(G, \widehat{G})$ and any well-covering pair $(C, \delta)$,

$$
\mu^{\mathcal{L}(\lambda \otimes \widehat{\lambda})}(C, \delta)=0 \Leftrightarrow X^{s}(\mathcal{L}(\lambda \otimes \widehat{\lambda})) \cap C \neq \varnothing .
$$

If $X^{s}(\mathcal{L}(\lambda \otimes \widehat{\lambda})) \cap C \neq \varnothing$, by Proposition8 (e), $\mu^{\mathcal{L}(\lambda \otimes \widehat{\lambda})}(C, \delta)=0$, since $C \subset X^{\delta}$. Conversely, if $\mu^{\mathcal{L}(\lambda \otimes \widehat{\lambda})}(C, \delta)=0$, take $x \in X^{s}(\mathcal{L}(\lambda \otimes \widehat{\lambda})) \cap C_{+}$(which is possible since $\operatorname{Im} \eta$ contains an open subset). By Proposition8 (c), $\lim _{t \rightarrow 0} \delta(t) \cdot x \in X^{s}(\mathcal{L}(\lambda \otimes \widehat{\lambda}))$. This proves (55).

From (55), we see that $F_{+} \cap \Lambda_{++} \times \widehat{\Lambda}_{++}$is a (finite) union of GIT classes. In particular, it contains a GIT class $\mathcal{C}\left(\lambda_{o}, \widehat{\lambda}_{o}\right)$ such that the cone generated by it has a nonempty interior in $F_{+}$. Take $x_{o} \in X^{s} \cap C$ such that its $G$-orbit is closed in $X^{s}$, where we have abbreviated $X^{s}:=X^{s}\left(\mathcal{L}\left(\lambda_{o} \otimes \widehat{\lambda}_{o}\right)\right)$ and $C:=C_{\delta}(w, \widehat{w})$. By the following argument, such an $x_{0}$ exists:

Take a $P(\delta)$-orbit $\mathfrak{O}:=P(\delta) \cdot x$ in $C_{+} \cap X^{s}$ of the smallest dimension. Then, $\mathfrak{O}$ is a closed subset of $X^{s}$; for if it is not closed in $X^{s}$, then its closure $\overline{\mathfrak{O}}$ in $X^{s}$ would contain a $P(\delta)$-orbit $\mathfrak{O}^{\prime}$ of strictly smaller dimension. Of course, $\mathfrak{D}^{\prime} \subset \bar{C}_{+}$, where $\bar{C}_{+}$is the closure of $C_{+}$in $X$. Further, $\partial C_{+}:=\bar{C}_{+} \backslash C_{+} \subset X \backslash X^{s}$. To see this, take a 


\section{SHRAWAN KUMAR}

$G$-equivariant embedding $\theta: X \hookrightarrow \mathbb{P}(V)$ for a $G$-module $V$ such that $\mathcal{L}\left(\lambda_{o} \otimes \widehat{\lambda}_{o}\right)^{N}$ is $G$-equivariantly isomorphic with $\theta^{*}(\mathcal{O}(1))$ for some $N>0$. Decompose $V=$ $V_{-} \oplus V_{0} \oplus V_{+}$under the action of $\delta(t)$, where $V_{0}$ is the invariant subspace and $V_{+}$ (resp. $V_{-}$) is the sum of the eigenspaces of positive (resp. negative) eigenvalues. Then, it is easy to see that $C \subset \mathbb{P}\left(V_{0}\right), C_{+} \subset \mathbb{P}\left(V_{0} \oplus V_{+}\right)$and $\partial C_{+} \subset \mathbb{P}\left(V_{+}\right)$. Thus, $\partial C_{+} \subset X \backslash X^{s}$. Hence, $\mathfrak{O}^{\prime} \subset C_{+}$, a contradiction, proving that $\mathfrak{O}$ is closed in $C_{+} \cap X^{s}$. By Lemma $9, \mu^{\mathcal{L}\left(\lambda_{o} \otimes \widehat{\lambda}_{o}\right)}\left(C_{+}, \delta\right)=0$. Hence, for any $x \in \mathfrak{O}$, by Proposition $8(\mathrm{c}), x_{o}:=\lim _{t \rightarrow 0} \delta(t) \cdot x \in X^{s}$. Thus, $x_{o} \in \mathfrak{O}$. Hence, $G \cdot x_{o}=G \cdot \mathfrak{O}$ is closed in $X^{s}$, since $G / P(\delta)$ is a projective variety.

Since $G \cdot x_{o}$ is contained in an affine open subset of $X^{s}\left(\mathcal{L}\left(\lambda_{o} \otimes \widehat{\lambda}_{o}\right)\right)$, by Matsushima's theorem, the isotropy $G_{x_{o}}$ is a reductive group contained of course in a Borel subgroup of $G$. Thus, $\operatorname{Im} \delta \subset G_{x_{o}} \subset H^{\prime}$, for some maximal torus $H^{\prime}$ of $G$.

But, since $x_{o} \in X^{s}\left(\mathcal{L}\left(\lambda_{o} \otimes \lambda_{o}\right)\right)$, it is easy to see that $\mathcal{L}\left(N \lambda_{o} \otimes N \widehat{\lambda}_{o}\right)_{\mid G \cdot x_{o}}$ is $G$-equivariantly trivial for some $N>0$. Thus, $\mathcal{C}\left(\lambda_{o}, \widehat{\lambda}_{o}\right)$ and hence $F_{+}$is contained in the kernel of the following map:

$$
\gamma:(\Lambda \times \widehat{\Lambda}) \otimes_{\mathbb{Z}} \mathbb{R} \rightarrow \operatorname{Pic}^{G}\left(G \cdot x_{o}\right) \otimes_{\mathbb{Z}} \mathbb{R} \simeq \Lambda\left(G_{x_{o}}^{o}\right) \otimes_{\mathbb{Z}} \mathbb{R},
$$

where $\Lambda\left(G_{x_{o}}^{o}\right)$ is the character group of the identity component $G_{x_{o}}^{o}$ of $G_{x_{o}}$. But, since $\gamma$ is clearly surjective and $F_{+}$lies in the kernel of $\gamma, \Lambda\left(G_{x_{o}}^{o}\right) \bigotimes_{\mathbb{Z}} \mathbb{R}$ is at most one-dimensional. Further, since $\operatorname{Im} \delta \subset G_{x_{o}}^{o}$, we see that $G_{x_{o}}^{o}$ is exactly onedimensional and $\operatorname{Im} \delta=G_{x_{o}}^{o}$. Thus, the general isotropy of the action of $L(\delta) / \operatorname{Im} \delta$ on $C_{\delta}(w, \widehat{w})$ is finite. As an $L(\delta)$-variety, $C_{\delta}(w, \widehat{w})$ is isomorphic with the full flag variety $(L(\delta) / B(\delta)) \times(\widehat{L}(\delta) / \widehat{B}(\delta))$ of $L(\delta) \times \widehat{L}(\delta)$, where $B(\delta)$ (resp. $\widehat{B}(\delta))$ is a Borel subgroup of $L(\delta)$ (resp. $\widehat{L}(\delta))$ containing $H$ (resp. $\widehat{H}$ ). Since $L(\delta)$ acts transitively on $L(\delta) / B(\delta)$ and centralizes $\delta$, for a general point $y \widehat{B}(\delta)$, the identity component of the isotropy of the point

$$
(B(\delta), y \widehat{B}(\delta)) \in(L(\delta) / B(\delta)) \times(\widehat{L}(\delta) / \widehat{B}(\delta))
$$

under the action of $L(\delta)$ coincides with $\operatorname{Im} \delta$. Let $\widehat{U}(\delta)$ be the unipotent radical of $\widehat{B}(\delta)$ and let $\widehat{w}_{o}^{\delta}$ be the longest element of the Weyl group of $\widehat{L}(\delta)$. Then, we have the open cell $\widehat{U}(\delta) \simeq \widehat{U}(\delta) \cdot \widehat{w}_{o}^{\delta} \widehat{B}(\delta) / \widehat{B}(\delta)$ in $\widehat{L}(\delta) / \widehat{B}(\delta)$. Replacing the point $(B(\delta), y \widehat{B}(\delta))$ by $(l B(\delta), l y \widehat{B}(\delta))$, for some $l \in L(\delta)$, we can assume that $B(\delta)=\widehat{B}(\delta) \cap L(\delta)$. Under the action of $B(\delta)$ on $\widehat{L}(\delta) / \widehat{B}(\delta)$, the open cell $\widehat{U}(\delta)$ is stable and the action is given by

$$
(t \cdot u) \widehat{u}=t u \widehat{u} t^{-1}, \text { for } t \in H, u \in U(\delta), \widehat{u} \in \widehat{U}(\delta),
$$

where $U(\delta)$ is the unipotent radical of $B(\delta)$. Since the isotropy of $(B(\delta), y \widehat{B}(\delta))$ under the action of $L(\delta)$ coincides with the isotropy of $y \widehat{B}(\delta)$ under the action of $B(\delta)$, for a general point $\widehat{u} \in \widehat{U}(\delta)$, the connected component of the isotropy of $U(\delta) \cdot \widehat{u} \in U(\delta) \backslash \widehat{U}(\delta)$ under the action of $H$ (via the conjugation action) coincides with $\operatorname{Im} \delta$. 


\section{EIGENVALUE PROBLEM}

But $U(\delta) \backslash \widehat{U}(\delta) \simeq \widehat{\mathfrak{u}}(\delta) / \mathfrak{u}(\delta)$ as $H$-varieties. Thus, we get $\mathbb{C} \dot{\delta}=\cap \operatorname{Ker} \beta$, where the intersection runs over all the $H$-weights $\beta$ of $\widehat{\mathfrak{u}}(\delta) / \mathfrak{u}(\delta)$. Thus, $\delta$ is special.

This proves that any facet of $\Gamma(G, \widehat{G})_{\mathbb{R}}$ which intersects $\Lambda_{++}(\mathbb{R}) \times \widehat{\Lambda}_{++}(\mathbb{R})$ is given by

$$
\lambda(w \dot{\delta})+\widehat{\lambda}(\widehat{w} \dot{\delta})=0,
$$

where $\left(C_{\delta}(w, \widehat{w}), \delta\right)$ is a well-covering pair with $\delta \in O(H)$ special.

Thus, the theorem follows from Proposition 31 and Lemma 32.

Remark 7. (a) Knutson-Tao [BS] proved a weaker version of Theorem 33, where they have (in general) many more inequalities. Their set of inequalities consists of $I_{(w, \widehat{w})}^{\delta}$, where $\delta$ runs over (in general) a larger set of OPS in $H$ than $\mathfrak{S}(G, \widehat{G})$ and for any $\delta$ in their set, they considered the inequalities $I_{(w, \widehat{w})}^{\delta}$ for any pair $(w, \widehat{w}) \in W^{P\left(\delta_{i}\right)} \times \widehat{W}^{\widehat{P}\left(\delta_{i}\right)}$ satisfying only $\iota^{*}\left(\left[\widehat{X}_{\widehat{w}}^{\widehat{P}\left(\delta_{i}\right)}\right]\right) \cdot\left[X_{w}^{P\left(\delta_{i}\right)}\right]=d\left[X_{e}^{P\left(\delta_{i}\right)}\right]$, for some nonzero $d$.

(b) The equivalence of (a) and (c) in Theorem 33 can also be obtained by a proof quite similar to the proof of Theorem 24 .

Lemma 34. If we specialize Theorem 33 to the case when $G$ is a connected semisimple group, $\widehat{G}=G^{s-1}$ and $G$ is embedded in $G^{s-1}$ diagonally, then we recover Theorem 24.

Proof. Since $\mathfrak{g}$ is semisimple, no nonzero ideal of $\mathfrak{g}$ is an ideal of $\widehat{\mathfrak{g}}:=\mathfrak{g}^{s-1}$. Further, the set of nonzero $H$-weights of $\widehat{\mathfrak{g}} / \mathfrak{g}$ is precisely equal to the set $R$ of the roots of g. Now, for a root $\beta$ and a (dominant) element $x=\sum_{p=1}^{k} r_{i_{p}} x_{i_{p}} \in \mathfrak{h}_{+}$with each $r_{i_{p}}>0$ and $i_{1}, \ldots, i_{k}$ distinct, $\beta(x)=0$ if and only if $\beta \in \sum_{j \notin\left\{i_{1}, \ldots, i_{k}\right\}} \mathbb{Z} \alpha_{j}$. Thus, if $k \geq 2$, then there is no OPS $\delta \in \mathfrak{S}(G, \widehat{G})$ such that $\dot{\delta}=x$. From this we see that $\mathfrak{S}(G, \widehat{G})=\left\{\delta_{i}(z):=z^{d_{i} x_{i}}\right\}_{1 \leq i \leq \ell}$, for some unique positive rational numbers $d_{i}$. Clearly, $\left\{P\left(\delta_{i}\right)\right\}_{1 \leq i \leq \ell}$ bijectively parameterizes the set of the standard maximal parabolic subgroups of $G$. By using the identity (31), it is easy to see that for $w=w_{1}, \widehat{w}=\left(w_{2}, \ldots, w_{s}\right)$, the identity $\left(\mathbf{b}_{2}\right)$ of Theorem 33 is equivalent to the identity

$$
\left(\left(\sum_{j=1}^{s} \chi_{w_{j}}\right)-\chi_{1}\right)\left(x_{i}\right)=0
$$

Thus, by Proposition 21, the two conditions $\left(b_{1}\right)$ and $\left(b_{2}\right)$ of Theorem 33 are equivalent to the condition (b) of Theorem 24 . Hence, Theorem 33, for the case of the diagonal embedding $G \rightarrow G^{s-1}$, is equivalent to Theorem 24 .

The following theorem (again due to Ressayre $\left[R_{1}\right]$ ) shows that the set of inequalities given by the (c) part of Theorem 33 is an irredundant system. As earlier, let $\Gamma(G, \widehat{G})_{\mathbb{R}}$ be the cone generated by $\Gamma(G, \widehat{G})$ inside the vector space $\Lambda(\mathbb{R}) \times \widehat{\Lambda}(\mathbb{R})$, where $\Lambda(\mathbb{R}):=\Lambda \otimes_{\mathbb{Z}} \mathbb{R}$.

Theorem 35. Following the assumptions of Theorem 33, the set of inequalities provided by the (c)-part of Theorem 33 is an irredundant system of inequalities describing the cone $\Gamma(G, \widehat{G})_{\mathbb{R}}$ inside $\Lambda_{+}(\mathbb{R}) \times \widehat{\Lambda}_{+}(\mathbb{R})$, i.e., the hyperplanes given by the equality in $I_{(w, \widehat{w})}^{\delta_{i}}$ are precisely those facets of the cone $\Gamma(G, \widehat{G})_{\mathbb{R}}$ which 


\section{SHRAWAN KUMAR}

intersect the interior of $\Lambda_{+}(\mathbb{R}) \times \widehat{\Lambda}_{+}(\mathbb{R})$, where $\Lambda_{+}(\mathbb{R})$ denotes the cone inside $\Lambda(\mathbb{R})$ generated by $\Lambda_{+}$.

Proof. First of all, the inequalities $I_{(w, \widehat{w})}^{\delta_{i}}($ as in $(50))$ for $\delta_{i}$ and $(w, \widehat{w})$ as in the (c)-part of Theorem 33 are pairwise distinct, even up to scalar multiples:

The stabilizer of $\dot{\delta}_{i}$ under the action of $W$ (resp. $\widehat{W}$ ) is precisely equal to the subgroup $W_{P\left(\delta_{i}\right)}$ (resp. $\left.\widehat{W}_{\widehat{P}\left(\delta_{i}\right)}\right)$. Let the pair $\left(w \dot{\delta}_{i}, \widehat{w} \dot{\delta}_{i}\right)=z\left(v \dot{\delta}_{i}, \widehat{v} \dot{\delta}_{i}\right)$, for some $1 \leq i \leq q, z \in \mathbb{R}$ and $(w, \widehat{w}) \neq(v, \widehat{v}) \in W^{P\left(\delta_{i}\right)} \times \widehat{W}^{\widehat{P}\left(\delta_{i}\right)}$ as in the (c)-part of Theorem 33. Then, it is easy to see that $z= \pm 1$. Moreover, $z \neq 1$ because of the stabilizer assertion as above. Further, $z \neq-1$, for otherwise $\Gamma(G, \widehat{G})$ would satisfy two inequalities with opposite signs, contradicting Lemma 30 .

Now, $\left(w \dot{\delta}_{i}, \widehat{w} \dot{\delta}_{i}\right)$ can not be equal to $z\left(v \dot{\delta}_{j}, \widehat{v} \dot{\delta}_{j}\right)$, for any $1 \leq i \neq j \leq q$ and $z \in \mathbb{R}$ : We can not have $z>0$ since each $\delta_{i}$ is indecomposable. For $z<0$, again $\Gamma(G, \widehat{G})$ would satisfy two inequalities with opposite signs.

Also, since each $\dot{\delta}_{i} \neq 0$, none of the hyperplanes $H_{(w, \widehat{w})}^{\delta_{i}}: \lambda\left(w \dot{\delta}_{i}\right)+\widehat{\lambda}\left(\widehat{w} \dot{\delta}_{i}\right)=0$ (given by the (c)-part of Theorem 33) is a face of the dominant chamber for the group $G \times \widehat{G}$.

We finally show that $H_{(w, \widehat{w})}^{\delta_{i}} \cap \Gamma(G, \widehat{G})_{\mathbb{R}}$ is a (codimension one) facet of $\Gamma(G, \widehat{G})_{\mathbb{R}}$ for any $\delta_{i} \in \mathfrak{S}(G, \widehat{G})$ and any $(w, \widehat{w}) \in W^{P\left(\delta_{i}\right)} \times \widehat{W}^{\widehat{P}\left(\delta_{i}\right)}$ as in the (c)-part of Theorem 33:

In the following, we abbreviate $\delta_{i}$ by $\delta$. Consider $\Gamma(C)_{\mathbb{R}} \subset \Lambda(\mathbb{R}) \times \widehat{\Lambda}(\mathbb{R})$, where $C=C_{\delta}(w, \widehat{w})$,

$$
\Gamma(C):=\left\{(\lambda, \widehat{\lambda}) \in \Lambda \times \widehat{\Lambda}: H^{0}\left(C, \mathcal{L}(N \lambda \otimes N \widehat{\lambda})_{\mid C}\right)^{G^{\delta}} \neq 0, \text { for some } N>0\right\}
$$

and $\Gamma(C)_{\mathbb{R}}$ is the cone inside $\Lambda(\mathbb{R}) \times \widehat{\Lambda}(\mathbb{R})$ generated by $\Gamma(C)$. We show that

$$
\langle\Gamma(C)\rangle=\left\langle H_{(w, \widehat{w})}^{\delta} \cap \Gamma(G, \widehat{G})\right\rangle,
$$

where $\langle\Gamma(C)\rangle$ (resp. $\left.\left\langle H_{(w, \widehat{w})}^{\delta} \cap \Gamma(G, \widehat{G})\right\rangle\right)$ denotes the $\mathbb{R}$-subspace of $\Lambda(\mathbb{R}) \times \widehat{\Lambda}(\mathbb{R})$ spanned by $\Gamma(C)\left(\operatorname{resp} . H_{(w, \widehat{w})}^{\delta} \cap \Gamma(G, \widehat{G})\right)$. We first show that

$$
H_{(w, \widehat{w})}^{\delta} \cap \Gamma(G, \widehat{G}) \subset \Gamma(C) .
$$

Take $(\lambda, \widehat{\lambda}) \in H_{(w, \widehat{w})}^{\delta} \cap \Gamma(G, \widehat{G})$. Then, by the proof of Theorem 33 (specifically, the part "Proof of $(\mathrm{a}) \Rightarrow(\mathrm{b})$ ") there exists a $G$-semistable point $x=(g B, \widehat{g} \widehat{B}) \in$ $X:=G / B \times \widehat{G} / \widehat{B}$ corresponding to the line bundle $\mathcal{L}(\lambda \otimes \widehat{\lambda})$ such that $x \in C_{+}:=$ $C_{\delta}(w, \widehat{w})_{+}$with

$$
\mu^{\mathcal{L}(\lambda \otimes \widehat{\lambda})}(x, \delta)=-\lambda(w \dot{\delta})-\widehat{\lambda}(\widehat{w} \dot{\delta})=0 .
$$

Since $x$ is a semistable point, there exists $N>0$ and a section $\sigma \in H^{0}(X, \mathcal{L}(N \lambda \otimes$ $N \widehat{\lambda}))^{G}$ such that $\sigma(x) \neq 0$. Hence, by Proposition 8 (c), $\sigma$ does not vanish at $\lim _{t \rightarrow 0} \delta(t) x$. Thus, $(\lambda, \widehat{\lambda}) \in \Gamma(C)$. 


\section{EIGENVALUE PROBLEM}

Conversely, take finitely many $\left(\lambda_{j}, \widehat{\lambda}_{j}\right) \in \Gamma(C)$ which $\mathbb{R}$-span $\langle\Gamma(C)\rangle$. We can assume (replacing $\left(\lambda_{j}, \widehat{\lambda}_{j}\right)$ by a multiple $\left.\left(N \lambda_{j}, N \widehat{\lambda}_{j}\right)\right)$ that $\mathcal{L}_{j}:=\mathcal{L}\left(\lambda_{j} \otimes \widehat{\lambda}_{j}\right)_{\mid C}$ has a nonzero $L(\delta)$-invariant section $\sigma_{j}$. We now show that $\sigma_{j}$ can be extended to a $G$-invariant rational section $\widehat{\sigma}_{j}$ of $\mathcal{L}\left(\lambda_{j} \otimes \widehat{\lambda}_{j}\right)$ on $X$ :

Extend the action of $L(\delta)$ on $C$ to an action of $P(\delta)$ on $C$ by demanding that the unipotent radical $U(\delta)$ of $P(\delta)$ acts trivially on $C$ (and hence on $\mathcal{L}_{j}$ ). (Observe that the standard action of $P(\delta)$ on $X$ does not keep $C$ stable in general, so this action is a different action of $P(\delta)$ on $C$.) It is easy to see that the map $\pi_{\delta}: C_{+} \rightarrow C$ (defined just above Definition 9 ) is $P(\delta)$-equivariant. Thus, we have a $G$-equivariant line bundle $G \times{ }^{P(\delta)} \pi_{\delta}^{*}\left(\mathcal{L}_{j}\right) \rightarrow G \times^{P(\delta)} C_{+}$. Also, we have a $G$ equivariant line bundle $\eta^{*}\left(\mathcal{L}\left(\lambda_{j} \otimes \widehat{\lambda}_{j}\right)\right)$ on $G \times{ }^{P(\delta)} C_{+}$. We claim that these two $G$-equivariant line bundles denoted respectively by $\mathcal{M}_{1}$ and $\mathcal{M}_{2}$ on $G \times{ }^{P(\delta)} C_{+}$ are $G$-equivariantly isomorphic:

To prove this, recall the well-known result that for any parabolic subgroup $P$ of $G$ and any $P$-variety $Y$, the map

$$
\operatorname{Pic}^{G}\left(G \times{ }^{P} Y\right) \rightarrow \operatorname{Pic}^{P}(Y), \quad \mathcal{L} \mapsto \mathcal{L}_{\left.\right|_{Y}}
$$

is an isomorphism, where $\operatorname{Pic}^{P}(Y)$ is the group of isomorphism classes of $P$ equivariant line bundles on $Y$ (cf., e.g., [ $\mathrm{R}_{1}$, Lemma 4]). Thus, we are reduced to showing that the two line bundles $\mathcal{M}_{1}$ and $\mathcal{M}_{2}$ restricted to $C_{+}$are isomorphic as $P(\delta)$-equivariant line bundles. Further, the restriction map $\gamma: \operatorname{Pic}^{P(\delta)}\left(C_{+}\right) \rightarrow$ $\operatorname{Pic}^{L(\delta)}(C)$ is an isomorphism: Since $\pi_{\delta}: C_{+} \rightarrow C$ is $P(\delta)$-equivariant (with the trivial action of $U(\delta)$ on $C$ ), the map $\gamma$ is injective. Moreover, since $\pi_{\delta}: C_{+} \rightarrow C$ is a $(L(\delta)$-equivariant) vector bundle (by a result of Bialynicki-Birula), the restriction map between the nonequivariant Picard groups: $\operatorname{Pic}\left(C_{+}\right) \rightarrow \operatorname{Pic}(C)$ is an isomorphism. Thus, for any $\mathcal{M} \in \operatorname{Pic}^{P(\delta)}\left(C_{+}\right)$, the $P(\delta)$-equivariant line bundle

$$
\mathcal{D}:=\mathcal{M}^{*} \otimes\left(\pi_{\delta}^{*}\left(\mathcal{M}_{\mid C}\right)\right)
$$

is nonequivariantly trivial. Hence, the $P(\delta)$-equivariant structure on the line bundle $\mathcal{D}$ is given by a character $\chi$ of $P(\delta)$. Further, since $\mathcal{D}$ restricted to $C$ is $L(\delta)$-equivariantly trivial, the character $\chi_{\left.\right|_{L(\delta)}}$ is trivial and hence so is $\chi$ itself. Hence, $\mathcal{D}$ is $P(\delta)$-equivariantly trivial, i.e., $\mathcal{M}_{1}$ and $\mathcal{M}_{2}$ are isomorphic as $P(\delta)$ equivariant line bundles. Thus, it suffices to show that the two line bundles $\mathcal{M}_{1}$ and $\mathcal{M}_{2}$ restricted to $C$ are $L(\delta)$-equivariantly isomorphic. But, this is true since both of the line bundles are the same restricted to $C$.

The $L\left(\delta\right.$ )-invariant section $\sigma_{j}$ of $\mathcal{L}_{j}$ (which is automatically $P(\delta)$-invariant) gives rise to the $G$-invariant section $\bar{\sigma}_{j}$ defined by $[g, x] \mapsto\left[g, \sigma_{j}\left(\pi_{\delta}(x)\right)\right]$, for $g \in G, x \in$ $C_{+}$. Since $(C, \delta)$ is a well-covering pair, $\bar{\sigma}_{j}$ descends to a $G$-invariant regular section on a $G$-stable open subset $X^{o}$ of $X$ such that $X^{o} \cap C \neq \varnothing$, and thus a $G$-invariant rational section $\widehat{\sigma}_{j}$ of the line bundle $\mathcal{L}\left(\lambda_{j} \otimes \widehat{\lambda}_{j}\right)$ on $X$. Let $\left\{E_{p}\right\}_{p}$ be the irreducible components of $X \backslash X^{o}$ of codimension one. Since $G$ is connected, each $E_{p}$ is $G$-stable. Consider the line bundle $\mathcal{E}:=\mathcal{O}_{X}\left(\sum_{p} a_{p} E_{p}\right)$, with $a_{p} \geq 0$ large enough so that each of the rational sections $\widehat{\sigma}_{j}$ of $\mathcal{L}\left(\lambda_{j} \otimes \widehat{\lambda}_{j}\right)$ are $(G$-invariant $)$ regular sections $\widehat{\lambda}_{j}^{o}$ of the line bundle $\mathcal{L}\left(\lambda_{j} \otimes \widehat{\lambda}_{j}\right) \otimes \mathcal{E}$. Moreover, since no $E_{p}$ contains 


\section{SHRAWAN KUMAR}

$C,\left(\widehat{\lambda}_{j}^{o}\right)_{\mid C} \neq 0$. We can easily lift the diagonal $G$-equivariant structure on $\mathcal{E}$ to a $G \times \widehat{G}$-equivariant structure by replacing (if needed) $\mathcal{E}$ by $\mathcal{E}^{N}$ for some $N>0$. Let $\mathcal{E} \simeq \mathcal{L}(\mu \otimes \widehat{\mu})$. Then, $\left(\lambda_{j}+\mu, \widehat{\lambda}_{j}+\widehat{\mu}\right) \in H_{(w, \widehat{w})}^{\delta} \cap \Gamma(G, \widehat{G})$, for all $j$. Since both of $\left(\lambda_{j}+\mu, \widehat{\lambda}_{j}+\widehat{\mu}\right)$ and $\left(\lambda_{j}+2 \mu, \widehat{\lambda}_{j}+2 \widehat{\mu}\right)$ are in $H_{(w, \widehat{w})}^{\delta} \cap \Gamma(G, \widehat{G})$, we see that each $\left(\lambda_{j}, \widehat{\lambda}_{j}\right) \in\left\langle H_{(w, \widehat{w})}^{\delta} \cap \Gamma(G, \widehat{G})\right\rangle$. Thus,

$$
\langle\Gamma(C)\rangle \subset\left\langle H_{(w, \widehat{w})}^{\delta} \cap \Gamma(G, \widehat{G})\right\rangle .
$$

Combining (57) and (58), we get (56).

As a $G^{\delta}$-variety, $C$ is isomorphic with $G^{\delta} / B^{\delta} \times \widehat{G}^{\delta} / \widehat{B}^{\delta}$. Thus, by [MR, Corollaire $1],\langle\Gamma(C)\rangle$ is of codimension one in $\Lambda(\mathbb{R}) \times \widehat{\Lambda}(\mathbb{R})$, since $\delta$ is special. This proves the theorem.

The following result for any semisimple and connected $G$ is a particular case of Theorem 35 (cf. Lemma 34). In the case $G=\mathrm{SL}(n)$, the following corollary was earlier proved by Knutson-Tao-Woodward [KTW].

Corollary 36. The set of inequalities provided by the (b)-part of Theorem 24 is an irredundant system of inequalities describing the cone $\Gamma_{s}(G)_{\mathbb{R}}$ generated by $\Gamma_{s}(G)$ inside $\Lambda_{+}(\mathbb{R})^{s}$, i.e., the hyperplanes given by the equality in $I_{\left(w_{1}, \ldots, w_{s}\right)}^{P}$ are precisely those facets of the cone $\Gamma_{s}(G)_{\mathbb{R}}$ which intersect the interior of $\Lambda_{+}(\mathbb{R})^{s}$.

By Theorem 5 , the same result is true for the cone $\bar{\Gamma}_{s}(\mathfrak{g})$, i.e., the inequalities given by Corollary 29(b) form an irredundant system of inequalities describing the cone $\bar{\Gamma}_{s}(\mathfrak{g})$ inside $\mathfrak{h}_{+}^{s}$, i.e., the hyperplanes given by the equality in $I_{\left(w_{1}, \ldots, w_{s}\right)}^{P}$ are precisely those facets of the cone $\bar{\Gamma}_{s}(\mathfrak{g})$ which intersect the interior of $\mathfrak{h}_{+}^{s}$.

Remark 8. (1) Fix a maximal compact subgroup $\widehat{K} \subset \widehat{G}$ and $K \subset G$ such that $K \subset \widehat{K}$. Define

$$
\bar{\Gamma}(\mathfrak{g}, \widehat{\mathfrak{g}}):=\left\{(h, \widehat{h}) \in \mathfrak{h}_{+} \times \widehat{\mathfrak{h}}_{+}: K \cdot(-h) \cap \pi(\widehat{K} \cdot \widehat{h}) \neq \varnothing\right\},
$$

where $\pi: \hat{i \mathfrak{k}} \rightarrow i \mathfrak{k}$ is the restriction map obtained from the identifications (induced from the Killing forms) $\widehat{i \mathfrak{k}} \simeq \widehat{\mathfrak{k}}^{*}$ and $i \mathfrak{k} \simeq i \mathfrak{k}^{*}$. Then, we get exact analogues of Theorems 33 and 35 for $\bar{\Gamma}(\mathfrak{g}, \widehat{\mathfrak{g}})$ by using an analogue of Theorem 5 in this setting (just as we got Corollary 29 from Theorem 24).

(2) Knutson-Tao have determined the cone $\bar{\Gamma}(\mathfrak{g}, \widehat{\mathfrak{g}})$ for the pairs $(\mathfrak{h}, \mathfrak{g}$ ) (for any semisimple $\mathfrak{g}$ and its Cartan subalgebra $\mathfrak{h}$ ); $\left(\mathfrak{s}, \mathfrak{g}\right.$ ) (for any $s l_{2}$-triple $\mathfrak{s}$ ); and $\left(G_{2}, \operatorname{sl}(3)\right)$ (cf. [BS, $\left.\left.\S 5\right]\right)$.

(3) Smaller faces of the cone $\Gamma(G, \widehat{G})_{\mathbb{R}}$ are determined by Ressayre in $\left[\mathrm{R}_{1}\right]$ and $\left[\mathrm{R}_{5}\right]$ (also see $[\mathrm{Br}]$ ).

(4) For any simple $G$ with Lie algebra different from $\mathfrak{s l}(2)$, the cone $\Gamma_{s}(G)_{\mathbb{R}}$ inside $\Lambda(\mathbb{R})^{s}$ has facets precisely those given by the facets of $\Gamma_{s}(G)_{\mathbb{R}}$ intersecting the interior of $\Lambda_{+}(\mathbb{R})^{s}$ together with the facets of the dominant chamber $\Lambda_{+}(\mathbb{R})^{s}$ inside $\Lambda(\mathbb{R})^{s}$ (cf. [KTW, Thm. 4] for $G=\mathrm{SL}(n), n \geq 3$, and [MR] for an arbitrary $G)$. As observed by Ressayre, it is easy to see that this property fails for the pair $(\mathrm{GL}(n), \mathrm{SL}(n+1))$ embedded as a Levi subgroup. 


\section{EIGENVALUE PROBLEM}

\section{Notational generalities on classical groups}

For a general reference for the material in this section, see, e.g., [BL]. In its present form it is taken from $\left[\mathrm{BK}_{2}\right]$.

\subsection{Special linear group $\operatorname{SL}(n+1)$.}

In this case we take $B$ to be the (standard) Borel subgroup consisting of upper triangular matrices of determinant 1 and $H$ to be the subgroup consisting of diagonal matrices (of determinant 1). Then,

$$
\mathfrak{h}=\left\{\mathbf{t}=\operatorname{diag}\left(t_{1}, \ldots, t_{n+1}\right): \sum t_{i}=0\right\},
$$

and

$$
\mathfrak{h}_{+}=\left\{\mathbf{t} \in \mathfrak{h}: t_{i} \in \mathbb{R} \text { and } t_{1} \geq \cdots \geq t_{n+1}\right\} .
$$

For any $1 \leq i \leq n$,

$$
\alpha_{i}(\mathbf{t})=t_{i}-t_{i+1} ; \quad \alpha_{i}^{\vee}=\operatorname{diag}(0, \ldots, 0,1,-1,0, \ldots, 0) ; \quad \omega_{i}(\mathbf{t})=t_{1}+\cdots t_{i},
$$

where 1 is placed in the $i$ th place.

The Weyl group $W$ can be identified with the symmetric group $S_{n+1}$, which acts via the permutation of the coordinates of $\mathbf{t}$. Let $\left\{r_{1}, \ldots, r_{n}\right\} \subset S_{n+1}$ be the (simple) reflections corresponding to the simple roots $\left\{\alpha_{1}, \ldots, \alpha_{n}\right\}$ respectively. Then,

$$
r_{i}=(i, i+1) .
$$

For any $1 \leq m \leq n$, let $P_{m} \supset B$ be the (standard) maximal parabolic subgroup of $\operatorname{SL}(n+1)$ such that its unique Levi subgroup $L_{m}$ containing $H$ has for its simple roots $\left\{\alpha_{1}, \ldots, \widehat{\alpha}_{m}, \ldots, \alpha_{n}\right\}$. Then, $\operatorname{SL}(n+1) / P_{m}$ can be identified with the Grassmannian $\operatorname{Gr}(m, n+1)=\operatorname{Gr}\left(m, \mathbb{C}^{n+1}\right)$ of $m$-dimensional subspaces of $\mathbb{C}^{n+1}$. Moreover, the set of minimal coset representatives $W^{P_{m}}$ of $W / W_{P_{m}}$ can be identified with the set of $m$-tuples

$$
S(m, n+1)=\left\{A:=1 \leq a_{1}<\cdots<a_{m} \leq n+1\right\} .
$$

Any such $m$-tuple $A$ represents the permutation

$$
v_{A}=\left(a_{1}, \ldots, a_{m}, a_{m+1}, \ldots, a_{n+1}\right),
$$

where $\left\{a_{m+1}<\cdots<a_{n+1}\right\}=[n+1] \backslash\left\{a_{1}, \ldots, a_{m}\right\}$ and

$$
[n+1]:=\{1, \ldots, n+1\} .
$$

For a complete flag $E_{\bullet}: 0=E_{0} \subsetneq E_{1} \subsetneq \cdots \subsetneq E_{n+1}=\mathbb{C}^{n+1}$, and $A \in$ $S(m, n+1)$, define the corresponding shifted Schubert cell inside $\operatorname{Gr}(m, n+1)$ :

$$
\begin{aligned}
\Omega_{A}\left(E_{\bullet}\right)=\{M \in \operatorname{Gr}(m, n+1) & \text { for any } 0 \leq \ell \leq m \text { and any } a_{\ell} \leq b<a_{\ell+1}, \\
& \left.\operatorname{dim} M \cap E_{b}=\ell\right\},
\end{aligned}
$$

where we set $a_{0}=0$ and $a_{m+1}=n+1$. Then, $\Omega_{A}\left(E_{\bullet}\right)=g\left(E_{\bullet}\right) C_{v_{A}}^{P_{m}}$, where $g\left(E_{\bullet}\right)$ is an element of SL $(n+1)$ which takes the standard flag $E_{\bullet}^{o}$ to the flag $E_{\bullet}$. (Observe that $g\left(E_{\bullet}\right)$ is determined up to the right multiplication by an element of $B$.) Its closure in $\operatorname{Gr}(m, n+1)$ is denoted by $\bar{\Omega}_{A}\left(E_{\bullet}\right)$ and its cycle class in $H^{*}(\operatorname{Gr}(m, n+1))$ by $\left[\bar{\Omega}_{A}\right]$. (Observe that the cohomology class $\left[\bar{\Omega}_{A}\right]$ does not depend upon the choice of $E_{\bullet}$.) For the standard flag $E_{\bullet}=E_{\bullet}^{o}$, we thus have $\Omega_{A}\left(E_{\bullet}\right)=C_{v_{A}}^{P_{m}}$. 


\section{SHRAWAN KUMAR}

\subsection{Symplectic group $\operatorname{Sp}(2 n)$.}

Let $V=\mathbb{C}^{2 n}$ be equipped with the nondegenerate symplectic form $\langle$,$\rangle so that its$ matrix $\left(\left\langle e_{i}, e_{j}\right\rangle\right)_{1 \leq i, j \leq 2 n}$ in the standard basis $\left\{e_{1}, \ldots, e_{2 n}\right\}$ is given by

$$
E=\left(\begin{array}{cc}
0 & J \\
-J & 0
\end{array}\right)
$$

where $J$ is the anti-diagonal matrix $(1, \ldots, 1)$ of size $n$. Let

$$
\operatorname{Sp}(2 n):=\{g \in \mathrm{SL}(2 n): g \text { leaves the form }\langle,\rangle \text { invariant }\}
$$

be the associated symplectic group. Clearly, $\operatorname{Sp}(2 n)$ can be realized as the fixed point subgroup $G^{\sigma}$ under the involution $\sigma: G \rightarrow G$ defined by $\sigma(A)=E\left(A^{t}\right)^{-1} E^{-1}$, where $G=\operatorname{SL}(2 n)$. The involution $\sigma$ keeps both of $B$ and $H$ stable, where $B$ and $H$ are as in the $\mathrm{SL}(2 n)$ case. Moreover, $B^{\sigma}$ (respectively, $H^{\sigma}$ ) is a Borel subgroup (respectively, a maximal torus) of $\mathrm{Sp}(2 n)$. We denote $B^{\sigma}, H^{\sigma}$ by $B^{C}=B^{C_{n}}, H^{C}=$ $H^{C_{n}}$ respectively and (when confusion is likely) $B, H$ by $B^{A_{2 n-1}}, H^{A_{2 n-1}}$ respectively (for SL $(2 n)$ ). Then, the Lie algebra of $H^{C}$ (the Cartan subalgebra $\mathfrak{h}^{C}$ )

$$
\mathfrak{h}^{C}=\left\{\operatorname{diag}\left(t_{1}, \ldots, t_{n},-t_{n}, \ldots,-t_{1}\right): t_{i} \in \mathbb{C}\right\} .
$$

Let $\Delta^{C}=\left\{\beta_{1}, \ldots, \beta_{n}\right\}$ be the set of simple roots. Then, for any $1 \leq i \leq n, \beta_{i}=$ $\alpha_{i \mid \mathfrak{h}^{C}}$, where $\left\{\alpha_{1}, \ldots, \alpha_{2 n-1}\right\}$ are the simple roots of $\operatorname{SL}(2 n)$. The corresponding (simple) coroots $\left\{\beta_{1}^{\vee}, \ldots, \beta_{n}^{\vee}\right\}$ are given by

$$
\beta_{i}^{\vee}=\alpha_{i}^{\vee}+\alpha_{2 n-i}^{\vee}, \text { for } 1 \leq i<n
$$

and

$$
\beta_{n}^{\vee}=\alpha_{n}^{\vee}
$$

Thus,

$$
\mathfrak{h}_{+}^{C}=\left\{\operatorname{diag}\left(t_{1}, \ldots, t_{n},-t_{n}, \ldots,-t_{1}\right): \text { each } t_{i} \text { is real and } t_{1} \geq \cdots \geq t_{n} \geq 0\right\} .
$$

Moreover, $\mathfrak{h}_{+}^{A_{2 n-1}}$ is $\sigma$-stable and

$$
\left(\mathfrak{h}_{+}^{A_{2 n-1}}\right)^{\sigma}=\mathfrak{h}_{+}^{C} .
$$

Let $\left\{s_{1}, \ldots, s_{n}\right\}$ be the (simple) reflections in the Weyl group $W^{C}=W^{C_{n}}$ of $\operatorname{Sp}(2 n)$ corresponding to the simple roots $\left\{\beta_{1}, \ldots, \beta_{n}\right\}$ respectively. Since $H^{A_{2 n-1}}$ is $\sigma$-stable, there is an induced action of $\sigma$ on the Weyl group $S_{2 n}$ of $\operatorname{SL}(2 n)$. The Weyl group $W^{C}$ can be identified with the subgroup of $S_{2 n}$ consisting of $\sigma$-invariants:

$$
\left\{\left(a_{1}, \ldots, a_{2 n}\right) \in S_{2 n}: a_{2 n+1-i}=2 n+1-a_{i} \forall 1 \leq i \leq 2 n\right\} .
$$

In particular, $w=\left(a_{1}, \ldots, a_{2 n}\right) \in W^{C}$ is determined from $\left(a_{1}, \ldots, a_{n}\right)$. 
Under the inclusion $W^{C} \subset S_{2 n}$, we have

$$
\begin{aligned}
s_{i} & =r_{i} r_{2 n-i} \text { if } 1 \leq i \leq n-1 \\
& =r_{n} \text { if } i=n .
\end{aligned}
$$

Moreover, for any $u, v \in W^{C}$ such that $\ell^{C}(u v)=\ell^{C}(u)+\ell^{C}(v)$, we have

$$
\ell^{A_{2 n-1}}(u v)=\ell^{A_{2 n-1}}(u)+\ell^{A_{2 n-1}}(v)
$$

where $\ell^{C}(w)$ denotes the length of $w$ as an element of the Weyl group $W^{C}$ of $\operatorname{Sp}(2 n)$ and similarly for $\ell^{A_{2 n-1}}$.

For $1 \leq r \leq n$, we let $\operatorname{IG}(r, 2 n)=\operatorname{IG}(r, V)$ to be the set of $r$-dimensional isotropic subspaces of $V$ with respect to the form $\langle$,$\rangle , i.e.,$

$$
\mathrm{IG}(r, 2 n):=\left\{M \in \operatorname{Gr}(r, 2 n):\left\langle v, v^{\prime}\right\rangle=0, \forall v, v^{\prime} \in M\right\} .
$$

Then, it is the quotient $\operatorname{Sp}(2 n) / P_{r}^{C}$ of $\operatorname{Sp}(2 n)$ by the standard maximal parabolic subgroup $P_{r}^{C}$ with $\Delta^{C} \backslash\left\{\beta_{r}\right\}$ as the set of simple roots of its Levi component $L_{r}^{C}$. (Again we take $L_{r}^{C}$ to be the unique Levi subgroup of $P_{r}^{C}$ containing $H^{C}$.) It can be easily seen that the set $W_{r}^{C}$ of minimal-length coset representatives of $W^{C} / W_{P_{r}^{C}}$ is identified with the set

$$
\mathfrak{S}(r, 2 n)=\left\{I:=1 \leq i_{1}<\cdots<i_{r} \leq 2 n \text { and } I \cap \bar{I}=\varnothing\right\}
$$

where

$$
\bar{I}:=\left\{2 n+1-i_{1}, \ldots, 2 n+1-i_{r}\right\}
$$

Any such $I$ represents the permutation $w_{I}=\left(i_{1}, \ldots, i_{n}\right) \in W^{C}$ by taking $\left\{i_{r+1}<\right.$ $\left.\cdots<i_{n}\right\}=[n] \backslash(I \sqcup \bar{I})$.

\subsection{Definition}

A complete flag

$$
E_{\bullet}: 0=E_{0} \subsetneq E_{1} \subsetneq \cdots \subsetneq E_{2 n}=V
$$

is called an isotropic flag if $E_{a}^{\perp}=E_{2 n-a}$, for $a=1, \ldots, 2 n$. (In particular, $E_{n}$ is a maximal isotropic subspace of $V$.)

For an isotropic flag $E_{\bullet}$ as above, there exists an element $k\left(E_{\bullet}\right) \in \operatorname{Sp}(2 n)$ which takes the standard flag $E_{\bullet}^{o}$ to the flag $E_{\bullet}$. (Observe that $k\left(E_{\bullet}\right)$ is determined up to the right multiplication by an element of $B^{C}$.)

For any $I \in \mathfrak{S}(r, 2 n)$ and any isotropic flag $E_{\bullet}$, we have the corresponding shifted Schubert cell inside IG $(r, V)$ :

$\Phi_{I}\left(E_{\bullet}\right)=\left\{M \in \operatorname{IG}(r, V):\right.$ for any $0 \leq \ell \leq r$ and any $\left.i_{\ell} \leq a<i_{\ell+1}, \operatorname{dim} M \cap E_{a}=\ell\right\}$, where we set $i_{0}=0$ and $i_{r+1}=2 n$. Clearly, set theoretically,

$$
\Phi_{I}\left(E_{\bullet}\right)=\Omega_{I}\left(E_{\bullet}\right) \cap \mathrm{IG}(r, V)
$$

this is also a scheme theoretic equality (cf. $\left[\mathrm{BK}_{2}\right.$, Prop. $\left.\left.36(4)\right]\right)$. Moreover, $\Phi_{I}\left(E_{\bullet}\right)=$ $k\left(E_{\bullet}\right) C_{w_{I}}^{P_{r}^{C}}$. Denote the closure of $\Phi_{I}\left(E_{\bullet}\right)$ inside $\operatorname{IG}(r, V)$ by $\bar{\Phi}_{I}\left(E_{\bullet}\right)$ and its cycle class in $H^{*}(\operatorname{IG}(r, V)$ ) (which does not depend upon the choice of the isotropic flag $\left.E_{\bullet}\right)$ by $\left[\bar{\Phi}_{I}\right]$. For the standard flag $E_{\bullet}=E_{\bullet}^{o}$, we have $\Phi_{I}\left(E_{\bullet}\right)=C_{w_{I}^{r}}^{P^{C}}$. 


\section{SHRAWAN KUMAR}

\subsection{Special orthogonal group $\mathrm{SO}(2 n+1)$.}

Let $V^{\prime}=\mathbb{C}^{2 n+1}$ be equipped with the nondegenerate symmetric form $\langle$,$\rangle so that$ its matrix $E=\left(\left\langle e_{i}, e_{j}\right\rangle\right)_{1 \leq i, j<2 n+1}$ (in the standard basis $\left\{e_{1}, \ldots, e_{2 n+1}\right\}$ ) is the $(2 n+1) \times(2 n+1)$ antidiagonal matrix with 1 's all along the antidiagonal except at the $(n+1, n+1)$ th place where the entry is 2 . Note that the associated quadratic form on $V^{\prime}$ is given by

$$
Q\left(\sum t_{i} e_{i}\right)=t_{n+1}^{2}+\sum_{i=1}^{n} t_{i} t_{2 n+2-i}
$$

Let

$$
\mathrm{SO}(2 n+1):=\{g \in \mathrm{SL}(2 n+1): g \text { leaves the quadratic form } Q \text { invariant }\}
$$

be the associated special orthogonal group. Clearly, $\mathrm{SO}(2 n+1)$ can be realized as the fixed point subgroup $G^{\theta}$ under the involution $\theta: G \rightarrow G$ defined by $\theta(A)=$ $E^{-1}\left(A^{t}\right)^{-1} E$, where $G=\operatorname{SL}(2 n+1)$. The involution $\theta$ keeps both of $B$ and $H$ stable. Moreover, $B^{\theta}$ (respectively, $H^{\theta}$ ) is a Borel subgroup (respectively, a maximal torus) of $\mathrm{SO}(2 n+1)$. We denote $B^{\theta}, H^{\theta}$ by $B^{B}=B^{B_{n}}, H^{B}=H^{B_{n}}$ respectively. Then, the Lie algebra of $H^{B}$ (the Cartan subalgebra $\mathfrak{h}^{B}$ )

$$
\mathfrak{h}^{B}=\left\{\operatorname{diag}\left(t_{1}, \ldots, t_{n}, 0,-t_{n}, \ldots,-t_{1}\right): t_{i} \in \mathbb{C}\right\} .
$$

This allows us to identify $\mathfrak{h}^{C}$ with $\mathfrak{h}^{B}$ under the map

$$
\operatorname{diag}\left(t_{1}, \ldots, t_{n},-t_{n}, \ldots,-t_{1}\right) \mapsto \operatorname{diag}\left(t_{1}, \ldots, t_{n}, 0,-t_{n}, \ldots,-t_{1}\right) .
$$

Let $\Delta^{B}=\left\{\delta_{1}, \ldots, \delta_{n}\right\}$ be the set of simple roots. Then, for any $1 \leq i \leq n, \delta_{i}=$ $\alpha_{i \mid \mathfrak{h}^{B}}$, where $\left\{\alpha_{1}, \ldots, \alpha_{2 n}\right\}$ are the simple roots of $\mathrm{SL}(2 n+1)$. The corresponding (simple) coroots $\left\{\delta_{1}^{\vee}, \ldots, \delta_{n}^{\vee}\right\}$ are given by

$$
\delta_{i}^{\vee}=\alpha_{i}^{\vee}+\alpha_{2 n+1-i}^{\vee}, \text { for } 1 \leq i<n
$$

and

$$
\delta_{n}^{\vee}=2\left(\alpha_{n}^{\vee}+\alpha_{n+1}^{\vee}\right) .
$$

Thus, under the above identification,

$$
\mathfrak{h}_{+}^{B}=\mathfrak{h}_{+}^{C} .
$$

Moreover, $\mathfrak{h}_{+}^{A_{2 n}}$ is $\theta$-stable and

$$
\left(\mathfrak{h}_{+}^{A_{2 n}}\right)^{\theta}=\mathfrak{h}_{+}^{B} .
$$

Let $\left\{s_{1}^{\prime}, \ldots, s_{n}^{\prime}\right\}$ be the (simple) reflections in the Weyl group $W^{B}=W^{B_{n}}$ of $\mathrm{SO}(2 n+1)$ corresponding to the simple roots $\left\{\delta_{1}, \ldots, \delta_{n}\right\}$ respectively. Since $H^{A_{2 n}}$ is $\theta$-stable, there is an induced action of $\theta$ on the Weyl group $S_{2 n+1}$ of $\mathrm{SL}(2 n+1)$. 


\section{EIGENVALUE PROBLEM}

The Weyl group $W^{B}$ can be identified with the subgroup of $S_{2 n+1}$ consisting of $\theta$-invariants:

$$
\left\{\left(a_{1}, \ldots, a_{2 n+1}\right) \in S_{2 n+1}: a_{2 n+2-i}=2 n+2-a_{i} \forall 1 \leq i \leq 2 n+1\right\} .
$$

In particular, $w=\left(a_{1}, \ldots, a_{2 n+1}\right) \in W^{B}$ is determined from $\left(a_{1}, \ldots, a_{n}\right)$. (Observe that $a_{n+1}=n+1$.) We can identify the Weyl groups $W^{C} \simeq W^{B}$ under the map $\left(a_{1}, \ldots, a_{2 n}\right) \mapsto\left(a_{1}, \ldots, a_{n}, n+1, a_{n+1}+1, \ldots, a_{2 n}+1\right)$.

Under the inclusion $W^{B} \subset S_{2 n+1}$, we have

$$
\begin{aligned}
s_{i}^{\prime} & =r_{i} r_{2 n+1-i} \text { if } 1 \leq i \leq n-1, \\
& =r_{n} r_{n+1} r_{n} \text { if } i=n .
\end{aligned}
$$

For $1 \leq r \leq n$, we let $\mathrm{OG}(r, 2 n+1)=\mathrm{OG}\left(r, V^{\prime}\right)$ be the set of $r$-dimensional isotropic subspaces of $V^{\prime}$ with respect to the quadratic form $Q$, i.e.,

$$
\mathrm{OG}(r, 2 n+1):=\left\{M \in \operatorname{Gr}\left(r, V^{\prime}\right): Q(v)=0, \forall v \in M\right\} .
$$

Then, it is the quotient $\mathrm{SO}(2 n+1) / P_{r}^{B}$ of $\mathrm{SO}(2 n+1)$ by the standard maximal parabolic subgroup $P_{r}^{B}$ with $\Delta^{B} \backslash\left\{\delta_{r}\right\}$ as the set of simple roots of its Levi component $L_{r}^{B}$. (Again we take $L_{r}^{B}$ to be the unique Levi subgroup of $P_{r}^{B}$ containing $H^{B}$.) It can be easily seen that the set $W_{r}^{B}$ of minimal-length coset representatives of $W^{B} / W_{P_{r}^{B}}$ is identified with the set

$\mathfrak{S}^{\prime}(r, 2 n+1)=\left\{J:=1 \leq j_{1}<\cdots<j_{r} \leq 2 n+1, j_{p} \neq n+1\right.$ for any $p$ and $\left.J \cap \bar{J}^{\prime}=\varnothing\right\}$

where

$$
\bar{J}^{\prime}:=\left\{2 n+2-j_{1}, \ldots, 2 n+2-j_{r}\right\} .
$$

Any such $J$ represents the permutation $w_{J}^{\prime}=\left(j_{1}, \ldots, j_{n}\right) \in W^{B}$ by taking $\left\{j_{r+1}<\right.$ $\left.\cdots<j_{n}\right\}=[n] \backslash\left(J \sqcup \bar{J}^{\prime}\right)$.

Similar to the Definition 8.3 of isotropic flags on $V$, we have the notion of isotropic flags on $V^{\prime}$. Then, for an isotropic flag $E_{\bullet}^{\prime}$, there exists an element $k\left(E_{\bullet}^{\prime}\right) \in$ $\mathrm{SO}(2 n+1)$ which takes the standard flag $E^{\prime o}$ to the flag $E_{\bullet}^{\prime}$. (Observe that $k\left(E_{\bullet}^{\prime}\right)$ is determined up to the right multiplication by an element of $B^{B}$.)

For any $J \in \mathfrak{S}^{\prime}(r, 2 n+1)$ and any isotropic flag $E_{\bullet}^{\prime}$, we have the corresponding shifted Schubert cell inside OG $\left(r, V^{\prime}\right)$ :

$$
\begin{aligned}
& \Psi_{J}\left(E_{\bullet}^{\prime}\right)=\left\{M \in \mathrm{OG}\left(r, V^{\prime}\right):\right. \\
& \left.\qquad \text { for any } 0 \leq \ell \leq r \text { and any } j_{\ell} \leq a<j_{\ell+1}, \operatorname{dim} M \cap E_{a}^{\prime}=\ell\right\},
\end{aligned}
$$

where we set $j_{0}=0$ and $j_{r+1}=2 n+1$. Clearly, set theoretically,

$$
\Psi_{J}\left(E_{\bullet}^{\prime}\right)=\Omega_{J}\left(E_{\bullet}^{\prime}\right) \cap \mathrm{OG}\left(r, V^{\prime}\right) ;
$$

this is also a scheme theoretic equality. Moreover, $\Psi_{J}\left(E_{\bullet}^{\prime}\right)=k\left(E_{\bullet}^{\prime}\right) C_{w_{J}^{\prime}}^{P_{r}^{B}}$. Denote the closure of $\Psi_{J}\left(E_{\bullet}^{\prime}\right)$ inside $\mathrm{OG}\left(r, V^{\prime}\right)$ by $\bar{\Psi}_{J}\left(E_{\bullet}^{\prime}\right)$ and its cycle class in $H^{*}\left(\mathrm{OG}\left(r, V^{\prime}\right)\right)$ (which does not depend upon the choice of the isotropic flag $E_{\bullet}^{\prime}$ ) by $\left[\bar{\Psi}_{J}\right]$. For the standard flag $E_{\bullet}^{\prime}=E_{\bullet}^{o}$, we have $\Psi_{J}\left(E_{\bullet}^{\prime}\right)=C_{w_{J}^{\prime}}^{P_{r}^{B}}$. 


\section{SHRAWAN KUMAR}

\section{Comparison of the eigencones under diagram automorphisms}

Fix a positive integer $s$. Let $V=\mathbb{C}^{2 n}$ be equipped with the nondegenerate symplectic form $\langle$,$\rangle as in Section 8$, and let $1 \leq r \leq n$ be a positive integer. Let $A^{1}, \ldots, A^{s} \in \mathfrak{S}(r, 2 n)$. The following theorem is a key technical result that underlies the proof of the comparison of the eigencone for $\operatorname{Sp}(2 n)$ with that of $\mathrm{SL}(2 n)$. The following results $37-41$ are due to Belkale-Kumar $\left[\mathrm{BK}_{2}\right]$.

Instead of giving the original proof of the following theorem due to BelkaleKumar $\left[\mathrm{BK}_{2}\right]$, we give a shorter proof observed by Sottile [So] using the work of Eisenbud-Harris $[\mathrm{EH}]$ on rational normal curves.

Theorem 37. Let $E_{\bullet}^{1}, \ldots, E_{\bullet}^{s}$ be isotropic flags on $V$ in general position. Then, the intersection of subvarieties $\bigcap_{j=1}^{s} \bar{\Omega}_{A^{j}}\left(E_{\bullet}^{j}\right)$ inside $\operatorname{Gr}(r, V)$ is proper (possibly empty).

Proof. Consider the rational normal curve $\gamma: \mathbb{C} \rightarrow V=\mathbb{C}^{2 n}$,

$$
\gamma(t)=\left(1, t, \frac{t^{2}}{2 !}, \ldots, \frac{t^{n}}{n !},-\frac{t^{n+1}}{n+1 !}, \frac{t^{n+2}}{n+2 !}, \ldots,(-1)^{n-1} \frac{t^{2 n-1}}{2 n-1 !}\right)
$$

Defne the corresponding 'osculating' flag

$$
E(t) .: E(t)_{1} \subset \cdots \subset E(t)_{2 n}
$$

by $E(t)_{j}:=\mathbb{C} \gamma(t) \oplus \mathbb{C} \gamma^{(1)}(t) \oplus \cdots \oplus \mathbb{C} \gamma^{(j-1)}(t)$, where $\gamma^{(k)}(t)$ is the $k$ th derivative of $\gamma$ at $t$. Then, it is easy to see that $E(t)$. is an isotropic flag for any value of $t$.

By a theorem due to Eisenbud-Harris [EH, Thm. 2.3], the intersection

$$
\bigcap_{j=1}^{s} \bar{\Omega}_{A^{j}}\left(E\left(t_{j}\right) \bullet\right.
$$

inside $\operatorname{Gr}(r, V)$ is proper if $t_{1}, \ldots, t_{s}$ are distinct complex numbers. This proves the theorem.

Remark 9. Even though we do not need it, as observed by Sottile [So] using the work of Mukhin-Tarasov-Varchenko [MTV, Cor. 6.3], the intersection of the open cells $\bigcap_{j=1}^{s} \Omega_{A^{j}}\left(E\left(t_{j}\right)_{\bullet}\right)$ is transverse if $t_{1}, \ldots, t_{s}$ are distinct real numbers.

The following result follows as an immediate consequence of the above theorem.

Corollary 38. Let $1 \leq r \leq n$ and let $I^{1}, \ldots, I^{s} \in \mathfrak{S}(r, 2 n)$ be such that

$$
\prod_{j=1}^{s}\left[\bar{\Phi}_{I^{j}}\right] \neq 0 \in H^{*}(\mathrm{IG}(r, 2 n)) .
$$

Then, $\prod_{j=1}^{s}\left[\bar{\Omega}_{I^{j}}\right] \neq 0 \in H^{*}(\operatorname{Gr}(r, 2 n))$. 
Proof. Observe that by Proposition 7,

$$
\prod_{j=1}^{s}\left[\bar{\Phi}_{I^{j}}\right] \neq 0 \text { if and only if } \bigcap_{j=1}^{s} \bar{\Phi}_{I^{j}}\left(E_{\bullet}^{j}\right) \neq \varnothing
$$

for isotropic flags $\left\{E_{\bullet}^{j}\right\}$ such that the above intersection is proper. Thus, by assumption, $\bigcap_{j=1}^{s} \bar{\Phi}_{I^{j}}\left(E_{\bullet}^{j}\right) \neq \varnothing$ for such flags $\left\{E_{\bullet}^{j}\right\}$. By the above theorem and equation (62), we conclude that $\cap_{j=1}^{s} \bar{\Omega}_{I^{j}}\left(E_{\bullet}^{j}\right) \neq \varnothing$ and the intersection is proper for isotropic flags $\left\{E_{\bullet}^{j}\right\}_{1 \leq j \leq s}$ in general position. From this and using equation (65) for $\operatorname{Gr}(r, V)$, the corollary follows.

We have the following analogue of Theorem 37 for $\mathrm{SO}(2 n+1)$ proved similarly by replacing the rational normal curve $\gamma$ by $\eta: \mathbb{C} \rightarrow V=\mathbb{C}^{2 n+1}$ given by

$$
\eta(t)=\left(1, t, \frac{t^{2}}{2 !}, \ldots, \frac{t^{n-1}}{n-1 !}, \frac{t^{n}}{n ! \sqrt{2}},-\frac{t^{n+1}}{n+1 !}, \frac{t^{n+2}}{n+2 !}, \ldots,(-1)^{n} \frac{t^{2 n}}{2 n !}\right) .
$$

Theorem 39. Let $1 \leq r \leq n$. Let $A^{1}, \ldots, A^{s}$ be subsets of $[2 n+1]$ each of cardinality $r$. Let $E^{\prime 1}, \ldots,{E^{\prime}}^{s}$. be isotropic flags on $V^{\prime}=\mathbb{C}^{2 n+1}$ in general position. Then, the intersection $\bigcap_{j=1}^{s} \bar{\Omega}_{A^{j}}\left(E^{\prime j}\right.$. $)$ of subvarieties of $\operatorname{Gr}\left(r, V^{\prime}\right)$ is proper (possibly empty).

The following result follows as an immediate consequence of the above theorem (just as in the case of $\operatorname{Sp}(2 n)$ ).

Corollary 40. Let $1 \leq r \leq n$ and let $J^{1}, \ldots, J^{s} \in \mathfrak{S}^{\prime}(r, 2 n+1)$ be such that

$$
\prod_{j=1}^{s}\left[\bar{\Psi}_{J^{j}}\right] \neq 0 \in H^{*}(\mathrm{OG}(r, 2 n+1))
$$

Then, $\prod_{j=1}^{s}\left[\bar{\Omega}_{J^{j}}\right] \neq 0 \in H^{*}(\operatorname{Gr}(r, 2 n+1))$.

Recall that $\mathfrak{h}_{+}^{C}$ (respectively, $\mathfrak{h}_{+}^{B}$ ) is the dominant chamber in the Cartan subalgebra of $\operatorname{Sp}(2 n)$ (respectively, $\mathrm{SO}(2 n+1)$ ) as in Section 8 .

The following theorem provides a comparison of the eigencone for $s p(2 n)$ with that of $\operatorname{sl}(2 n)$ (and also for $s o(2 n+1)$ with that of $\operatorname{sl}(2 n+1)$ ).

Theorem 41. For any $s \geq 1$,

(a) $\bar{\Gamma}_{s}(s p(2 n))=\bar{\Gamma}_{s}(\operatorname{sl}(2 n)) \cap\left(\mathfrak{h}_{+}^{C}\right)^{s}$.

(b) $\bar{\Gamma}_{s}(s o(2 n+1))=\bar{\Gamma}_{s}(s l(2 n+1)) \cap\left(\mathfrak{h}_{+}^{B}\right)^{s}$.

(Observe that by Section $8, \mathfrak{h}_{+}^{C} \subset \mathfrak{h}_{+}^{A_{2 n-1}}$ and $\mathfrak{h}_{+}^{B} \subset \mathfrak{h}_{+}^{A_{2 n}}$ ).

Proof. Clearly, $\bar{\Gamma}_{s}(s p(2 n)) \subset \bar{\Gamma}_{s}(s l(2 n))$. Conversely, we need to show that if $\mathbf{h}=\left(h_{1}, \ldots, h_{s}\right) \in\left(\mathfrak{h}_{+}^{C}\right)^{s}$ is such that $\mathbf{h} \in \bar{\Gamma}_{s}(s l(2 n))$, then $\mathbf{h} \in \bar{\Gamma}_{s}(s p(2 n))$. Take any $1 \leq r \leq n$ and any $I^{1}, \ldots, I^{s} \in \mathfrak{S}(r, 2 n)$ such that

$$
\left[\bar{\Phi}_{I^{1}}\right] \ldots\left[\bar{\Phi}_{I^{s}}\right]=d\left[\bar{\Phi}_{e}\right] \in H^{*}(\operatorname{IG}(r, 2 n)) \text { for some nonzero } d \text {. }
$$


By Corollary 38,

$$
\left[\bar{\Omega}_{I^{1}}\right] \ldots\left[\bar{\Omega}_{I^{s}}\right] \neq 0 \in H^{*}(\operatorname{Gr}(r, 2 n)) .
$$

In particular, by Corollary 11 (rather Remark 1(a)) applied to $\operatorname{sl}(2 n)$,

$$
\omega_{r}\left(\sum_{j=1}^{s} v_{I^{j}}^{-1} h_{j}\right) \leq 0,
$$

where $\omega_{r}$ is the $r$ th fundamental weight of $\mathrm{SL}(2 n)$ and $v_{I^{j}} \in S_{2 n}$ is the element associated to $I^{j}$ as in Subsection 8.1. It is easy to see that the $r$ th fundamental weight $\omega_{r}^{C}$ of $\operatorname{Sp}(2 n)$ is the restriction of $\omega_{r}$ to $\mathfrak{h}^{C}$. Moreover, even though the elements $v_{I^{j}} \in S_{2 n}$ and $w_{I^{j}} \in W^{C}$ are, in general, different, we still have

$$
\omega_{r}\left(v_{I^{j}}^{-1} h_{j}\right)=\omega_{r}^{C}\left(w_{I^{j}}^{-1} h_{j}\right) .
$$

Applying Corollary 11 for $s p(2 n)$, we get the (a)-part of the theorem. 38.)

The proof for $s o(2 n+1)$ is similar. (Apply Corollary 40 instead of Corollary

Remark 10. (1) Belkale-Kumar have given a set of necessary and sufficient conditions to determine the nonvanishing of any product of Schubert classes $\left[X_{w}^{P}\right]$ in $\left(H^{*}(G / P), \odot_{0}\right)$ (under the deformed product) for any maximal parabolic subgroup $P$ and any $G$ of type $\mathrm{B}_{n}$ or $\mathrm{C}_{n}$ (cf. $\left[\mathrm{BK}_{2}\right.$, Thm. 30, 41 and Rem. 31, 42]).

(2) For any $G$ of type $\mathrm{B}_{n}$ or $\mathrm{C}_{n}$, and any maximal parabolic subgroup $P$, Ressayre has determined the triples $\left(w_{1}, w_{2}, w_{3}\right) \in\left(W^{P}\right)^{3}$ such that $\left[X_{w_{1}}^{P}\right] \odot_{0}$ $\left[X_{w_{2}}^{P}\right] \odot_{0}\left[X_{w_{3}}^{P}\right]=1\left[X_{e}^{P}\right]$ in terms of the corresponding result for certain associated Schubert varieties in Grassmannians (cf. $\left[\mathrm{R}_{3}\right.$, Thm. 14 and 15]).

Let $\mathfrak{g}$ be a simple simply-laced Lie algebra and let $\sigma: \mathfrak{g} \rightarrow \mathfrak{g}$ be a diagram automorphism with fixed subalgebra $\mathfrak{k}$ (which is necessarily a simple Lie algebra again). Let $\mathfrak{b}$ (resp. $\mathfrak{h}$ ) be a Borel (resp. Cartan) subalgebra of $\mathfrak{g}$ such that they are stable under $\sigma$. Then, $\mathfrak{b}^{\mathfrak{k}}:=\mathfrak{b}^{\sigma}\left(\right.$ resp. $\left.\mathfrak{h}^{\mathfrak{k}}:=\mathfrak{h}^{\sigma}\right)$ is a Borel (resp. Cartan) subalgebra of $\mathfrak{k}$. Let $\mathfrak{h}_{+}$and $\mathfrak{h}_{+}^{\mathfrak{k}}$ be the dominant chambers in $\mathfrak{h}$ and $\mathfrak{h}^{\mathfrak{k}}$ respectively. Then,

$$
\mathfrak{h}_{+}^{\mathfrak{k}}=\mathfrak{h}_{+} \cap \mathfrak{k} .
$$

We have the following generalization of Theorem 41 conjectured by Belkale-Kumar. (In fact, they have made a stronger conjecture, cf. Conjecture 51.)

Theorem 42. For any $s \geq 1$,

$$
\bar{\Gamma}_{s}(\mathfrak{k})=\bar{\Gamma}_{s}(\mathfrak{g}) \cap\left(\mathfrak{h}_{+}^{\mathfrak{k}}\right)^{s} .
$$

(In the cases $(\mathrm{d})$ and $(\mathrm{e})$ as below, the theorem is proved only for $s=3$, though it must be true for any $s$ ).

Proof. Unfortunately, the proof is case by case. Following is the complete list of $(\mathfrak{g}, \mathfrak{k})$ coming from the diagram automorphisms of simple Lie algebras $\mathfrak{g}$ :
(a) $(\operatorname{sl}(2 n), \operatorname{sp}(2 n)), n \geq 2$,
(b) $(\operatorname{sl}(2 n+1)$, so $(2 n+1)), n \geq 2$,
(c) $(s o(2 n), s o(2 n-1)), n \geq 4$,
(d) $\left(s o(8), \mathrm{G}_{2}\right)$,
(e) $\left(\mathrm{E}_{6}, \mathrm{~F}_{4}\right)$. 


\section{EIGENVALUE PROBLEM}

In the cases (a) and (b), the theorem is nothing but Theorem 41.

In the case (c), it was proved by E. Braley in her thesis [Bra]. Similar to the proof of Theorem 41, her proof relies on the comparison between the intersection theory of the partial flag varieties $G / P$ of $G$ (corresponding to the maximal parabolic subgroups $P$ of $G$ ) with that of the partial flag varieties $K / Q$ of $K$ (corresponding to the maximal parabolic subgroups $Q$ of $K$ ). But her proof uses the deformed product in the cohomology of $K / Q$ and Corollary 29, whereas she needs to use the standard cup product in the cohomology of $G / P$ and Corollary 11.

The theorem for the cases (d) and (e) was proved by B. Lee in his thesis [Le]. Lee used the comparison between the deformed product in the cohomology of $G / P$ corresponding to the maximal parabolic subgroups $P$ of $G$ with that of the deformed product in the cohomology of $K / Q$ corresponding to the maximal parabolic subgroups $Q$ of $K$ (and Corollary 29). Lee used the recipe of Duan (cf. [D $\left.\mathrm{D}_{1}\right],\left[\mathrm{D}_{2}\right]$ ) to develop a program which allowed him to explicitly calculate the deformed product in the cohomology of the relevant flag varieties.

\section{Saturation problem}

We continue to follow the notation and assumptions from Section 2; in particular, $G$ is a semisimple connected complex algebraic group. In Section 3, we defined the saturated tensor semigroup $\Gamma_{s}(G)$ (for any integer $s \geq 1$ ) and determined it by describing its facets (cf. Theorems 10 and 24 ).

Define the tensor semigroup for $G$ :

$$
\widehat{\Gamma}_{s}(G)=\left\{\left(\lambda_{1}, \ldots, \lambda_{s}\right) \in \Lambda_{+}^{s}:\left[V\left(\lambda_{1}\right) \otimes \cdots \otimes V\left(\lambda_{s}\right)\right]^{G} \neq 0\right\} .
$$

It is indeed a semigroup by $\left[\mathrm{K}_{3}\right.$, Lemma 3.9]. The saturation problem aims at comparing these two semigroups. We first prove that $\widehat{\Gamma}_{s}(G)$ is a finitely generated semigroup. More generally, we have the following result (cf. [Br, Thm. 2.1]).

Lemma 43. Let $S$ be a reductive subgroup of a connected semisimple group $G$. Let

$$
\mathcal{D}_{S}=\left\{\lambda \in \Lambda_{+}:[V(\lambda)]^{S} \neq 0\right\},
$$

where $\Lambda_{+}$is the set of dominant characters of $G$. Then, $\mathcal{D}_{S}$ is a finitely generated semigroup.

Proof. Since $S$ is reductive, by Matsushima's theorem, $G / S$ is an affine variety. In particular, the affine coordinate ring $\mathbb{C}[G / S]$ is a finitely generated $\mathbb{C}$-algebra. Now, by the Frobenius reciprocity,

$$
\begin{aligned}
\mathbb{C}[G / S] & \simeq \bigoplus_{\lambda \in \Lambda_{+}} V(\lambda) \otimes\left[V(\lambda)^{*}\right]^{S} \text { as } G \text {-modules, } \\
& =\bigoplus_{\lambda \in \mathcal{D}_{S}} V(\lambda) \otimes\left[V(\lambda)^{*}\right]^{S} .
\end{aligned}
$$

Of course, $\mathbb{C}[G / S] \hookrightarrow \mathbb{C}[G]$. Consider the map $\Delta^{*}: \mathbb{C}[G] \otimes \mathbb{C}[G] \rightarrow \mathbb{C}[G]$ induced from the diagonal map $\Delta: G \rightarrow G \times G$. Then, for the $G \times G$-isotypic component 


\section{SHRAWAN KUMAR}

$V(\lambda) \otimes V(\lambda)^{*}$ of $\mathbb{C}[G]$, we have

$$
\Delta^{*}\left(\left(V(\lambda) \otimes V(\lambda)^{*}\right) \otimes\left(V(\mu) \otimes V(\mu)^{*}\right)\right) \subset V(\lambda+\mu) \otimes V(\lambda+\mu)^{*} .
$$

Take a finite set of algebra generators $f_{1}, \ldots, f_{N}$ of $\mathbb{C}[G / S]$ so that, under the above decomposition (66), $f_{p} \in V\left(\lambda_{p}\right) \otimes\left[V\left(\lambda_{p}\right)^{*}\right]^{S}$ for some $\lambda_{p} \in \mathcal{D}_{S}$. Then, it is easy to see that these $\left\{\lambda_{p}\right\}$ generate the semigroup $\mathcal{D}_{S}$.

As an easy consequence of the above lemma, we get the following.

Corollary 44. There exists a uniform integer $d>0$ (depending only upon $s$ and $G)$ such that for any $\lambda=\left(\lambda_{1}, \ldots, \lambda_{s}\right) \in \Gamma_{s}(G), d \lambda=\left(d \lambda_{1}, \ldots, d \lambda_{s}\right) \in \widehat{\Gamma}_{s}(G)$.

Proof. Take a finite set of semigroup generators $\lambda^{p}=\left(\lambda_{1}^{p}, \ldots, \lambda_{s}^{p}\right)$ of $\Gamma_{s}(G)$, which exists by Theorem 5 , since $\bar{\Gamma}_{s}(\mathfrak{g})$ is a rational polyhedral cone. Also, choose a finite set of semigroup generators $\mu^{k}=\left(\mu_{1}^{k}, \ldots, \mu_{s}^{k}\right)$ of $\widehat{\Gamma}_{s}(G)$ (cf. Lemma 43). We can of course write

$$
\lambda^{p}=\sum_{k} a_{p}^{k} \mu^{k}, \text { for some non-negative rational numbers } a_{p}^{k} .
$$

Now, take $d>0$ large enough so that $d a_{p}^{k} \in \mathbb{Z}_{+}$for all $a_{p}^{k}$. Take any $\gamma=$ $\left(\gamma_{1}, \ldots, \gamma_{s}\right) \in \Gamma_{s}(G)$ and write

$$
\begin{aligned}
\gamma & =\sum_{p} n_{p} \lambda^{p}, \text { for some } n_{p} \in \mathbb{Z}_{+} \\
& =\sum_{k, p} n_{p} a_{p}^{k} \mu^{k} \\
& =\sum_{k}\left(\sum_{p} n_{p} a_{p}^{k}\right) \mu^{k} .
\end{aligned}
$$

This implies that

$$
d \gamma=\sum_{k}\left(\sum_{p} n_{p} d a_{p}^{k}\right) \mu^{k} \in \widehat{\Gamma}_{s}(G) .
$$

We now begin with the following definition. We take $s=3$ as this is the most relevant case to the tensor product decomposition.

Definition 11. An integer $d \geq 1$ is called a saturation factor for $G$, if for any $(\lambda, \mu, \nu) \in \Gamma_{3}(G)$ such that $\lambda+\mu+\nu \in Q$, we have $(d \lambda, d \mu, d \nu) \in \widehat{\Gamma}_{3}(G)$, where $Q$ is the root lattice of $G$. Of course, if $d$ is a saturation factor then so is its any multiple. If $d=1$ is a saturation factor for $G$, we say that the saturation property holds for $G$.

The saturation theorem of Knutson-Tao (cf. Theorem 13) asserts that the saturation property holds for $G=\operatorname{SL}(n)$.

The following general result (though not optimal) on the saturation factor is obtained by Kapovich-Millson $\left[\mathrm{KM}_{2}\right]$ by using the geometry of geodesics in Euclidean buildings and Littelmann's path model (see the Appendix). A weaker form of the following theorem was conjectured by Kumar in a private communication to J. Millson (also see [KT, Conj.]). 


\section{EIGENVALUE PROBLEM}

Theorem 45. For any connected simple $G, d=k_{\mathfrak{g}}^{2}$ is a saturation factor, where $k_{\mathfrak{g}}$ is the least common multiple of the coefficients of the highest root $\theta$ of the Lie algebra $\mathfrak{g}$ of $G$ written in terms of the simple roots $\left\{\alpha_{1}, \ldots, \alpha_{\ell}\right\}$.

Observe that the value of $k_{\mathfrak{g}}$ is 1 for $\mathfrak{g}$ of type $\mathrm{A}_{\ell}(\ell \geq 1)$; it is 2 for $\mathfrak{g}$ of type $\mathrm{B}_{\ell}(\ell \geq 2), \mathrm{C}_{\ell}(\ell \geq 3), \mathrm{D}_{\ell}(\ell \geq 4)$; and it is $6,12,60,12,6$ for $\mathfrak{g}$ of type $\mathrm{E}_{6}, \mathrm{E}_{7}, \mathrm{E}_{8}$, $\mathrm{F}_{4}, \mathrm{G}_{2}$ respectively.

Kapovich-Millson determined $\widehat{\Gamma}_{3}(G)$ explicitly for $G=\operatorname{Sp}(4)$ and $\mathrm{G}_{2}$ (cf. $\left[\mathrm{KM}_{1}\right.$, Thm. 5.3, 6.1]). In particular, from their description, the following theorem follows easily.

Theorem 46. The saturation property does not hold for either $G=\operatorname{Sp}(4)$ or $\mathrm{G}_{2}$. Moreover, 2 is a saturation factor (and no odd integer $d$ is a saturation factor) for $\mathrm{Sp}(4)$, whereas both of 2,3 are saturation factors for $\mathrm{G}_{2}$ (and hence any integer $d>1$ is a saturation factor for $\mathrm{G}_{2}$ ).

It was known earlier that the saturation property fails for $G$ of type $B_{\ell}$ (cf. [E]). Kapovich-Millson $\left[\mathrm{KM}_{1}\right]$ made the following very interesting conjecture:

Conjecture 47. If $G$ is simply-laced, then the saturation property holds for $G$.

Apart from $G=\mathrm{SL}(n)$, the only other simply connected, simple, simply-laced group $G$ for which the above conjecture is known so far is $G=\operatorname{Spin}(8)$, proved by Kapovich-Kumar-Millson [KKM, Thm. 5.3] by explicit calculation using Theorem 24.

Theorem 48. The above conjecture is true for $G=\operatorname{Spin}(8)$.

Finally, we have the following improvement of Theorem 45 for the classical groups $\mathrm{SO}(n)$ and $\mathrm{Sp}(2 \ell)$. It was proved by Belkale-Kumar $\left[\mathrm{BK}_{2}\right.$, Thm. 25 and 26 ] for the groups $\mathrm{SO}(2 \ell+1)$ and $\mathrm{Sp}(2 \ell)$ by using geometric techniques. Sam [S] proved it for $\mathrm{SO}(2 \ell)$ (and also for $\mathrm{SO}(2 \ell+1)$ and $\mathrm{Sp}(2 \ell)$ ) via the quiver approach (following the proof by Derksen-Weyman [DW] for $G=\mathrm{SL}(n)$ ).

Theorem 49. For the groups $\mathrm{SO}(n)(n \geq 7)$ and $\mathrm{Sp}(2 \ell)(\ell \geq 2), 2$ is a saturation factor.

The Belkale-Kumar proof of the above theorem for $\mathrm{SO}(2 \ell+1)$ and $\mathrm{Sp}(2 \ell)$ relies on the following theorem $\left[\mathrm{BK}_{2}\right.$, Thm. 23].

Theorem 50. Let $\left(\lambda^{1}, \ldots, \lambda^{s}\right) \in \widehat{\Gamma}_{s}(\operatorname{SL}(2 \ell))$. Then, $\left(\lambda_{C}^{1}, \ldots, \lambda_{C}^{s}\right) \in \widehat{\Gamma}_{s}(\operatorname{Sp}(2 \ell))$, where $\lambda_{C}^{j}$ is the restriction of $\lambda^{j}$ to the maximal torus of $\operatorname{Sp}(2 \ell)$.

$A$ similar result is true for $\mathrm{Sp}(2 \ell)$ replaced by $\mathrm{SO}(2 \ell+1)$.

Belkale-Kumar $\left[\mathrm{BK}_{2}\right.$, Conj. 29] conjectured the following generalization of Theorem 50. Let $G$ be a simply-connected, simple complex algebraic group and let $\sigma$ be a diagram automorphism of $G$ (in particular, $G$ is simply-laced) with fixed subgroup $K$.

Conjecture 51. Let $\left(\lambda^{1}, \ldots, \lambda^{s}\right) \in \widehat{\Gamma}_{s}(G)$. Then, $\left(\lambda_{K}^{1}, \ldots, \lambda_{K}^{s}\right) \in \widehat{\Gamma}_{s}(K)$, where $\lambda_{K}^{j}$ is the restriction of $\lambda^{j}$ to the maximal torus of $K$.

(Observe that $\lambda_{K}$ is dominant for $K$ for any dominant character $\lambda$ for $G$ with respect to the Borel subgroup $B^{K}:=B^{\sigma}$ of $\left.K\right)$. 


\section{SHRAWAN KUMAR}

Remark 11. Lee showed in his thesis [Le] that the above conjecture is true for the pair (Spin (8), $\mathrm{G}_{2}$ ).

We generalize Definition 11 in the following.

Definition 12. Let $G \subset \widehat{G}$ be connected reductive groups with the choice of $H, \widehat{H}, B, \widehat{B}$ as in the beginning of Section 7 . An integer $d \geq 1$ is called a saturation factor for the pair $(G, \widehat{G})$, if for any $\widehat{\lambda} \in \widehat{\Lambda}_{+}$and $\lambda \in \Lambda_{+}$such that

(a) for all $t \in \widehat{Z} \cap G, \lambda(t) \cdot \widehat{\lambda}(t)=1$, where $\widehat{Z}$ is the center of $\widehat{G}$, and

(b) there exists $N>0$ such that $[V(N \lambda) \otimes \widehat{V}(N \widehat{\lambda})]^{G} \neq 0$,

then we have $[V(d \lambda) \otimes \widehat{V}(d \widehat{\lambda})]^{G} \neq 0$.

If we can take $d=1$, we say that the saturation property holds for the pair $(G, \widehat{G})$.

As proved by Pasquier-Ressayre [PS, Thm. 5], the pairs

$$
\begin{gathered}
(\operatorname{Spin}(2 n-1), \operatorname{Spin}(2 n)) ;\left(\operatorname{SL}(3), G_{2}\right) ;\left(G_{2}, \operatorname{Spin}(7)\right) ; \\
\left(\operatorname{Spin}(9), F_{4}\right) ;\left(F_{4}, E_{6}\right) ;(\operatorname{Sp}(2 n), \operatorname{SL}(2 n))
\end{gathered}
$$

for any $2 \leq n \leq 5$ have the saturation property.

The following result is due to Haines-Kapovich-Millson [HKM, Cor. 3.4], though we give a different proof (observed by A. Berenstein), reducing the problem to that of the saturation factor for $\widehat{G}$.

Theorem 52. Let $\widehat{G}$ be any connected simple group and $G$ any Levi subgroup. Then, if $d$ is a saturation factor for $\widehat{G}$, then $d$ is also a saturation factor for the pair $(G, \widehat{G})$.

In particular, $k_{\widehat{\mathfrak{g}}}^{2}$ is a saturation factor for the pair $(G, \widehat{G})$, where $\widehat{\mathfrak{g}}$ is the Lie algebra of $\widehat{G}$ and $k_{\widehat{\mathfrak{g}}}$ is as defined in Theorem 45.

Proof. Let $\omega_{G}:=\sum_{\alpha_{i} \in \widehat{\Delta} \backslash \Delta} \omega_{i}$. We first show that for any $\lambda \in \Lambda_{+}$and $\widehat{\lambda} \in \widehat{\Lambda}_{+}$,

$$
\operatorname{dim}\left([V(\lambda) \otimes \widehat{V}(\widehat{\lambda})]^{G}\right)=\operatorname{dim}\left(\left[\widehat{V}(\widehat{\lambda}) \otimes \widehat{V}\left(m \omega_{G}\right) \otimes \widehat{V}\left(-\widehat{w}_{o}\left(m \omega_{G}-w_{o} \lambda\right)\right)\right]^{\widehat{G}}\right),
$$

where $\widehat{w}_{o}$ (resp. $w_{o}$ ) is the longest element of the Weyl group $\widehat{W}$ of $\widehat{G}$ (resp. $W$ of $G)$ and $m=m_{\lambda, \widehat{\lambda}}$ is any positive integer such that $e_{i}^{m+1} \cdot x=0$, for all $x \in \widehat{V}(\widehat{\lambda})$ and $\alpha_{i} \in \widehat{\Delta} \backslash \Delta$ and such that $m \omega_{G}-w_{o} \lambda \in \widehat{\Lambda}_{+}$(where $e_{i}$ is the root vector corresponding to the simple root $\alpha_{i}$ ). To prove this, observe that (since $V(\lambda)^{*} \simeq$ $\left.V\left(-w_{o} \lambda\right)\right)$

$$
\begin{aligned}
{[V(\lambda) \otimes \widehat{V}(\widehat{\lambda})]^{G} } & \simeq \operatorname{Hom}_{G}\left(V\left(-w_{o} \lambda\right), \widehat{V}(\widehat{\lambda})\right) \\
& \simeq\left\{v \in \widehat{V}(\widehat{\lambda})_{-w_{o} \lambda}: e_{i} \cdot v=0 \text { for all } \alpha_{i} \in \Delta\right\} \\
& \simeq\left\{v \in \widehat{V}(\widehat{\lambda})_{-w_{o} \lambda}: e_{i}^{m\left\langle\omega_{G}, \alpha_{i}^{\vee}\right\rangle+1} \cdot v=0 \text { for all } \alpha_{i} \in \widehat{\Delta}\right\} .
\end{aligned}
$$

The last space has the same dimension as that of $\left[\widehat{V}(\widehat{\lambda}) \otimes \widehat{V}\left(m \omega_{G}\right) \otimes \widehat{V}\left(-\widehat{w}_{o}\left(m \omega_{G}\right.\right.\right.$ $\left.\left.-w_{o} \lambda\right)\right)^{\widehat{G}}$ from $\left[\mathrm{K}_{3}\right.$, Thm. 3.7]. This proves the identity (67). From the identity (67), the theorem follows easily by observing that $m_{N \lambda, N \widehat{\lambda}}$ can be taken to be $N m_{\lambda, \widehat{\lambda}}$. 
Example 1 (due to J. Millson). Let $p, r$ be positive integers and let $V=\mathbb{C}^{2 p+r}$ be equipped with a quadratic form $f$ that is the sum of $p$ hyperbolic planes and $r$ squares. Let $V=E \oplus W \oplus F$ be a Witt splitting, so $E$ and $F$ are totally isotropic $p$-dimensional subspaces which are dually paired by $f$ and $W$ has dimension $r$ with $f_{\left.\right|_{W}}$ nondegenerate. Let $G$ be the identity component of the isometry group of $f$, $P$ the stabilizer of $E$ in $G$ and $L$ the Levi subgroup of $P$ (i.e., $L \simeq \mathrm{GL}(E) \times \mathrm{SO}(W)$ is the stabilizer in $G$ of both $E$ and $F)$.

Then, the restriction of $V=V\left(\omega_{1}\right)$ to $L$ has no nonzero invariant vectors, whereas the restriction of $V\left(2 \omega_{1}\right)$ to $L$ has a nonzero invariant vector.

Remark 12. As shown by Roth [Ro] (also by Ressayre $\left[\mathrm{R}_{7}\right]$ ), for a pair $(\lambda, \hat{\lambda})$ in any regular face (i.e., a face which intersects $\Lambda_{++} \times \widehat{\Lambda}_{++}$) of the cone $\Gamma(G, \widehat{G})_{\mathbb{R}}$ (cf. Theorem 35 for the definition of $\Gamma(G, \widehat{G})_{\mathbb{R}}$ ), the dimension of the invariant subspace $[V(\lambda) \otimes \widehat{V}(\widehat{\lambda})]^{G}$ is equal to a similar dimension for representations of Levi subgroups of $G$ and $\widehat{G}$.

We also recall the following 'rigidity' result conjectured by Fulton and proved by Knutson-Tao-Woodward [KTW]. (Subsequently, geometric proofs were given by Belkale $\left[\mathrm{B}_{4}\right]$ and Ressayre $\left[\mathrm{R}_{2}\right]$.)

Theorem 53. Let $L=\mathrm{GL}(r)$ and let $\lambda, \mu, \nu \in \Lambda(H)_{+}$. Then, if the dimension $\operatorname{dim}\left([V(\lambda) \otimes V(\mu) \otimes V(\nu)]^{\mathrm{SL}(r)}\right)=1$, we have $\operatorname{dim}\left([V(n \lambda) \otimes V(n \mu) \otimes V(n \nu)]^{\mathrm{SL}(r)}\right)=$ 1 , for every positive integer $n$.

The direct generalization of the above theorem for an arbitrary connected reductive group $L$ is false. However, Belkale-Kumar-Ressayre [BKR] proved the following generalization using the deformed product.

Theorem 54. Let $G$ be any connected reductive group and let $P$ be any standard parabolic subgroup with the Levi subgroup $L$ containing $H$. Then, for any $w_{1}, \ldots, w_{s} \in W^{P}$ such that

$$
\left[X_{w_{1}}^{P}\right] \odot_{0} \cdots \odot_{0}\left[X_{w_{s}}^{P}\right]=\left[X_{e}^{P}\right] \in H^{*}\left(G / P, \odot_{0}\right),
$$

we have, for every positive integer $n$,

$$
\operatorname{dim}\left(\left[V_{L}\left(n \chi_{w_{1}}\right) \otimes \cdots \otimes V_{L}\left(n \chi_{w_{s}}\right)\right]^{L^{s s}}\right)=1,
$$

where $L^{s s}$ denotes the semisimple part $[L, L]$ of $L, V_{L}(\lambda)$ is the irreducible representation of $L$ with highest weight $\lambda$ and $\chi_{w}:=\rho-2 \rho^{L}+w^{-1} \rho$ ( $\rho$ and $\rho^{L}$ being the half sum of positive roots of $G$ and $L$ respectively).

\section{Deformed product and Lie algebra cohomology}

We continue to follow the same notation and assumptions from Section 2. We relate the cohomology algebra $H^{*}(G / P)$ under the product $\odot_{0}$ with the Lie algebra cohomology of the nil-radical $\mathfrak{u}_{P}$ of the parabolic subalgebra $\mathfrak{p}$. 


\section{SHRAWAN KUMAR}

For any Lie algebra $\mathfrak{s}$ and a subalgebra $\mathfrak{t}$, let $H^{*}(\mathfrak{s}, \mathfrak{t})$ be the Lie algebra cohomology of the pair $(\mathfrak{s}, \mathfrak{t})$ with trivial coefficients. Recall (cf. $\left[K_{1}\right.$, Sect. 3.1]) that this is the cohomology of the cochain complex

$$
\begin{aligned}
C^{\bullet}(\mathfrak{s}, \mathfrak{t}) & =\left\{C^{p}(\mathfrak{s}, \mathfrak{t})\right\}_{p \geq 0}, \quad \text { where } \\
C^{p}(\mathfrak{s}, \mathfrak{t}) & :=\operatorname{Hom}_{\mathfrak{t}}\left(\wedge^{p}(\mathfrak{s} / \mathfrak{t}), \mathbb{C}\right) .
\end{aligned}
$$

For any (positive) root $\beta \in R^{+}$, let $y_{\beta} \in \mathfrak{g}_{\beta}$ be a nonzero root vector and let $y_{-\beta} \in \mathfrak{g}_{-\beta}$ be the vector such that $\left\langle y_{\beta}, y_{-\beta}\right\rangle=1$ under the Killing form. For any $w \in W^{P}$, let $\Phi_{w}:=w^{-1} R^{-} \cap R^{+} \subset R\left(\mathfrak{u}_{P}\right)$. Then, as is well known,

$$
\sum_{\beta \in \Phi_{w}} \beta=\rho-w^{-1} \rho .
$$

In particular, $\Phi_{v}=\Phi_{w}$ iff $v=w$. Let $\Phi_{w}=\left\{\beta_{1}, \ldots, \beta_{p}\right\} \subset R\left(\mathfrak{u}_{P}\right)$. Set $y_{w}:=$ $y_{\beta_{1}} \wedge \cdots \wedge y_{\beta_{p}} \in \wedge^{p}\left(\mathfrak{u}_{P}\right)$, determined up to a nonzero scalar multiple. Then, up to scalar multiples, $y_{w}$ is the unique weight vector of $\wedge\left(\mathfrak{u}_{P}\right)$ with weight $\rho-w^{-1} \rho$ (cf. [Ko, Lemma 5.12]). Similarly, we can define $y_{w}^{-}:=y_{-\beta_{1}} \wedge \cdots \wedge y_{-\beta_{p}} \in \wedge^{p}\left(\mathfrak{u}_{P}^{-}\right)$ of weight $w^{-1} \rho-\rho$.

We recall the following fundamental result due to Kostant [Ko].

Theorem 55. For any standard parabolic subgroup $P$ of $G$,

$$
H^{p}\left(\mathfrak{u}_{P}\right)=\bigoplus_{\substack{w \in W^{P}: \\ \ell(w)=p}} M_{w},
$$

as $\mathfrak{l}_{P}$-modules, where $M_{w}$ is the unique irreducible $\mathfrak{l}_{P}$-submodule of $H^{p}\left(\mathfrak{u}_{P}\right)$ with highest weight $w^{-1} \rho-\rho$ (which is $\mathfrak{l}_{P}$-dominant for any $\left.w \in W^{P}\right)$. This has a highest weight vector $\phi_{w} \in \wedge^{p}\left(\mathfrak{u}_{P}\right)^{*}$ defined by $\phi_{w}\left(y_{w}\right)=1$ and $\phi_{w}(y)=0$ for any weight vector of $\wedge^{p}\left(\mathfrak{u}_{P}\right)$ of weight $\neq \rho-w^{-1} \rho$.

Similarly, for the opposite nil-radical $\mathfrak{u}_{P}^{-}$,

$$
H^{p}\left(\mathfrak{u}_{P}^{-}\right)=\bigoplus_{\substack{w \in W^{P}: \\ \ell(w)=p}} N_{w},
$$

as $\mathfrak{l}_{P}$-modules, where $N_{w}$ is the unique irreducible $\mathfrak{l}_{P}$-submodule of $H^{p}\left(\mathfrak{u}_{P}^{-}\right)$isomorphic with the dual $M_{w}^{*}$ and it has a lowest weight vector $\phi_{w}^{-} \in \wedge^{p}\left(\mathfrak{u}_{P}^{-}\right)^{*}$ defined by $\phi_{w}^{-}\left(y_{w}^{-}\right)=1$ and $\phi_{w}^{-}(y)=0$ for any weight vector of $\wedge^{p}\left(\mathfrak{u}_{P}^{-}\right)$of weight $\neq w^{-1} \rho-\rho$.

Thus,

$$
\begin{aligned}
& {\left[H^{p}\left(\mathfrak{u}_{P}\right) \otimes H^{q}\left(\mathfrak{u}_{P}^{-}\right)\right]^{\mathfrak{l}_{P}}=0, \quad \text { unless } p=q, \text { and }} \\
& {\left[H^{p}\left(\mathfrak{u}_{P}\right) \otimes H^{p}\left(\mathfrak{u}_{P}^{-}\right)\right]^{\mathfrak{l}_{P}} \simeq \bigoplus_{\substack{w \in W^{P}: \\
\ell(w)=p}} \mathbb{C} \xi^{w},}
\end{aligned}
$$

where $\xi^{w} \in\left[M_{w} \otimes N_{w}\right]^{l_{P}}$ is the unique element whose $H$-equivariant projection to $\left(M_{w}\right)_{w^{-1} \rho-\rho} \otimes N_{w}$ is the element $\phi_{w} \otimes \phi_{w}^{-},\left(M_{w}\right)_{w^{-1} \rho-\rho}$ being the weight space of $M_{w}$ corresponding to the weight $w^{-1} \rho-\rho$. (Observe that the ambiguity in the choice of $y_{w}$ disappears in the definition of $\xi^{w}$ giving rise to a completely unique element.)

The following theorem is due to Belkale-Kumar (cf. $\left[\mathrm{BK}_{1}\right.$, Thm. 43] for a proof). 
Theorem 56. For any standard parabolic subgroup $P$ of $G$, there is a graded algebra isomorphism

$$
\phi:\left(H^{*}(G / P, \mathbb{C}), \odot_{0}\right) \simeq\left[H^{*}\left(\mathfrak{u}_{P}\right) \otimes H^{*}\left(\mathfrak{u}_{P}^{-}\right)\right]^{\mathfrak{l}_{P}}
$$

such that, for any $w \in W^{P}$,

$$
\phi\left(\epsilon_{w}^{P}\right)=(-1)^{\frac{p(p-1)}{2}}\left(\frac{i}{2 \pi}\right)^{p}\left\langle\rho, \Phi_{w^{-1}}\right\rangle \xi^{w}
$$

where $p:=\ell(w),\left\langle\rho, \Phi_{w^{-1}}\right\rangle:=\prod_{\alpha \in w R^{-} \cap R^{+}}\langle\rho, \alpha\rangle$ (for any $\left.w \in W\right)$, and we take the tensor product algebra structure on the right side.

A proof of the following corollary due to Belkale-Kumar can be found in $\left[\mathrm{BK}_{1}\right.$, Cor. 44].

Corollary 57. The product in $\left(H^{*}(G / B), \odot_{0}\right)$ is given by

$$
\begin{aligned}
\epsilon_{u}^{B} \odot_{0} \epsilon_{v}^{B} & =0 \text { if } \Phi_{u} \cap \Phi_{v} \neq \varnothing \\
& =0 \text { if } \Phi_{u} \cap \Phi_{v}=\varnothing \text { and } \nexists w \in W \text { with } \Phi_{w}=\Phi_{u} \sqcup \Phi_{v} \\
& =\frac{\left\langle\rho, \Phi_{u^{-1}}\right\rangle\left\langle\rho, \Phi_{v^{-1}}\right\rangle}{\left\langle\rho, \Phi_{w^{-1}}\right\rangle} \epsilon_{w}^{B} \text { if } \Phi_{u} \cap \Phi_{v}=\varnothing \text { and } \exists w \in W \text { with } \Phi_{w}=\Phi_{u} \sqcup \Phi_{v} .
\end{aligned}
$$

As shown by Dimitrov-Roth [DR 1 , Thm. 9.1.2], for any classical $G$ or $G=G_{2}$, and any $u, v, w \in W$ such that $\Phi_{w}=\Phi_{u} \sqcup \Phi_{v}$, the structure constant

$$
\frac{\left\langle\rho, \Phi_{u^{-1}}\right\rangle\left\langle\rho, \Phi_{v^{-1}}\right\rangle}{\left\langle\rho, \Phi_{w^{-1}}\right\rangle}=1
$$

Remark 13. (a) The above result (Theorem 56) identifying $H^{*}(G / P)$ under the deformed product with the Lie algebra cohomology has crucially been used (though for affine $G$ ) by Kumar in the solution of the Cachazo-Douglas-Seiberg-Witten conjecture (cf. $\left[\mathrm{K}_{2}\right]$ ).

(b) Evens-Graham have realized the algebra $\left(H^{*}(G / P), \odot_{t}\right)$ (for any value of

$$
\left.t=\left(t_{1}, \ldots, t_{m}\right) \in \mathbb{C}^{m}, \text { where } m:=|\Delta|-|\Delta(P)|\right)
$$

as the relative cohomology algebra $H^{*}\left(\mathfrak{g}_{t}, \mathfrak{l}_{\Delta}\right)$ for certain Lie subalgbebras $\mathfrak{g}_{t} \supset \mathfrak{l}_{\Delta}$ of $\mathfrak{g} \times \mathfrak{g}\left(\mathrm{cf} .\left[\mathrm{EG}_{1}\right]\right)$.

Let $J_{t}:=\left\{\alpha_{q}, 1 \leq q \leq \ell: \alpha_{q} \in \Delta \backslash \Delta(P)\right.$ and $\left.t_{q} \neq 0\right\}, D_{t}:=\Delta(P) \cup J_{t}$ and let $P_{t} \supset P$ be the parabolic subgroup of $G$ such that its Levi subgroup has $D_{t}$ for its set of simple roots.

Now, Evens-Graham prove that the standard singular cohomology algebra $H^{*}\left(P_{t} / P\right)$ is isomorphic, as an algebra, to a certain graded subalgebra $A_{t}$ of $\left(H^{*}(G / P), \odot_{t}\right)$. Moreover, the algebra $\left(H^{*}\left(G / P_{t}\right), \odot_{0}\right)$ is isomorphic, as an algebra, with $\left(H^{*}(G / P), \odot_{t}\right) / I_{+}$, where $I_{+}$is the graded ideal of $\left(H^{*}(G / P), \odot_{t}\right)$ generated by the positive degree elements in $A_{t}$ (cf. $\left.\left[\mathrm{EG}_{2}\right]\right)$. 


\section{SHRAWAN KUMAR}

\section{A restricted functoriality of the deformed product and a product formula}

Let the notation and assumptions be as in the beginning of Section 7. Take a $G$ dominant OPS $\delta \in O(H)$. Thus, $P(\delta)$ is a standard parabolic since $\delta$ is dominant for $G$. Moreover, the choice of the Borel subgroup $\widehat{B}$ is made so that $B \subset \widehat{B} \supset \widehat{H}$ and $\widehat{B} \subset \widehat{P}(\delta)$. (Such a $\widehat{B}$ depends upon the choice of $\delta$.) We have the embedding $\iota: G / P(\delta) \rightarrow \widehat{G} / \widehat{P}(\delta)$. Define a $\mathbb{Z}[\tau]$-linear product $\odot^{\delta}$ (with single indeterminate $\tau)$ in $H^{*}(G / P, \mathbb{Z}) \otimes_{\mathbb{Z}} \mathbb{Z}[\tau]$ by

$$
\left.\left[X_{u}^{P}\right] \odot^{\delta}\left[X_{v}^{P}\right]=\sum_{w \in W^{P}} \tau^{\left(w^{-1} \rho-u^{-1} \rho-v^{-1} \rho-\rho\right)(\dot{\delta})}\right) c_{u, v}^{w}\left[X_{w}^{P}\right],
$$

where $P:=P(\delta)$ and $c_{u, v}^{w}$ is given by

$$
\left[X_{u}^{P}\right] \cdot\left[X_{v}^{P}\right]=\sum_{w \in W^{P}} c_{u, v}^{w}\left[X_{w}^{P}\right] .
$$

By Corollary 22, the exponent $\left(w^{-1} \rho-u^{-1} \rho-v^{-1} \rho-\rho\right)(\dot{\delta}) \geq 0$, whenever $c_{u, v}^{w} \neq 0$, since $\dot{\delta} \in \sum_{\alpha_{i} \in \Delta \backslash \Delta(P)} \mathbb{Z}_{+} x_{i}$.

Define a similar product, again denoted by $\odot^{\delta}$, in $H^{*}(\widehat{G} / \widehat{P}, \mathbb{Z}) \otimes_{\mathbb{Z}} \mathbb{Z}[\tau]$, where $\widehat{P}:=\widehat{P}(\delta)$.

In particular, we can specialize $\tau=0$ in the above product $\odot{ }^{\delta}$. Since $\alpha_{i}(\dot{\delta})>0$ for any $\alpha_{i} \in \Delta \backslash \Delta(P)$, it is easy to see from Corollary 22 that

$$
\left(\left[X_{u}^{P}\right] \odot^{\delta}\left[X_{v}^{P}\right]\right)_{\tau=0}=\left[X_{u}^{P}\right] \odot_{0}\left[X_{v}^{P}\right] .
$$

A similar result is true for the product $\odot^{\delta}$ in $H^{*}(\widehat{G} / \widehat{P}, \mathbb{Z})$. Let $\iota^{*}: H^{*}(\widehat{G} / \widehat{P}, \mathbb{Z}) \rightarrow$ $H^{*}(G / P, \mathbb{Z})$ be the standard pull-back map in cohomology. Write

$$
\iota^{*}\left(\left[\widehat{X}_{\widehat{w}}^{\widehat{P}}\right]\right)=\sum_{w \in W^{P}} d_{w}^{\widehat{w}}\left[X_{w}^{P}\right] .
$$

Now, define a $\mathbb{Z}[\tau]$-linear map

$$
\theta^{\delta}: H^{*}(\widehat{G} / \widehat{P}, \mathbb{Z}) \otimes_{\mathbb{Z}} \mathbb{Z}[\tau] \rightarrow H^{*}(G / P, \mathbb{Z}) \otimes_{\mathbb{Z}} \mathbb{Z}[\tau]
$$

by

$$
\theta^{\delta}\left(\left[\widehat{X}_{\widehat{w}}^{\widehat{P}}\right]\right)=\sum_{w \in W^{P}} \tau^{\chi_{w}(\dot{\delta})-\widehat{\chi}_{\hat{w}}(\dot{\delta})} d_{w}^{\widehat{w}}\left[X_{w}^{P}\right],
$$

where $\chi_{w}$ is given by the identity (31). By an argument similar to the proof of Corollary 22, we can see that if $d_{w}^{\widehat{w}} \neq 0$, then $\chi_{w}(\dot{\delta})-\widehat{\chi}_{\widehat{w}}(\dot{\delta}) \geq 0$. Thus, the map $\theta^{\delta}$ is well defined.

Let $\theta_{0}^{\delta}: H^{*}(\widehat{G} / \widehat{P}, \mathbb{Z}) \rightarrow H^{*}(G / P, \mathbb{Z})$ be the map obtained by setting $\tau=0$ in the definition of $\theta^{\delta}$. Let us express

$$
\theta_{0}^{\delta}\left(\left[\widehat{X}_{\widehat{w}}^{\widehat{P}}\right]\right)=\sum_{w \in W^{P}} c_{w}^{\widehat{w}}\left[X_{w}^{P}\right] .
$$

We have the following result due to Ressayre-Richmond [ReR, Thm. 1.1]. 
Theorem 58. The map $\theta_{0}^{\delta}: H^{*}(\widehat{G} / \widehat{P}, \mathbb{Z}) \rightarrow H^{*}(G / P, \mathbb{Z})$ is a graded algebra homomorphism with respect to the deformed products on both the domain and the range. Moreover, it satisfies

$$
c_{w}^{\widehat{w}}=d_{w}^{\widehat{w}} \text { if } c_{w}^{\widehat{w}} \neq 0 .
$$

Proof. It is easy to see, by an explicit calculation, that $\theta^{\delta}$ is a graded $\mathbb{Z}[\tau]$-algebra homomorphism with respect to the products $\odot^{\delta}$ on both the domain and the range. From this and the identity (72), the theorem follows immediately.

Remark 14. (1) As observed by Ressayre-Richmond [ReR, Lemma 3.3], it is easy to see that if $G / P$ is cominuscule, then $\theta_{0}^{\delta}=\iota^{*}$. (Use the identity (39), the definition of $\widehat{\chi}_{\widehat{w}}$ as in the identity (30) and the nonnegativity of $\chi_{w}(\dot{\delta})-\widehat{\chi}_{\widehat{w}}(\dot{\delta})$ if $d_{w}^{\widehat{w}} \neq 0$.)

(2) The map $\theta_{0}^{\delta}$ is partially computed for the pairs $(\operatorname{SL}(2), \operatorname{SL}(n)),(\operatorname{SL}(n) \times$ $\left.\mathrm{SL}(n), \mathrm{SL}\left(n^{2}\right)\right)$ and $(\mathrm{SO}(2 n+1), \mathrm{SL}(2 n+1))$ by Ressayre-Richmond [ReR, $\left.\S 4\right]$.

(3) Clearly, the conditions $\left(c_{1}\right)$ and $\left(c_{2}\right)$ in Theorem 33 can be replaced by the condition

$$
\left[X_{w}^{P\left(\delta_{i}\right)}\right] \cdot \theta_{0}^{\delta_{i}}\left(\left[\widehat{X}_{\widehat{w}}^{\widehat{P}\left(\delta_{i}\right)}\right]\right)=\left[X_{e}^{P\left(\delta_{i}\right)}\right] \in H^{*}\left(G / P\left(\delta_{i}\right), \mathbb{Z}\right),
$$

cf. [ReR, Thm. 5.1].

We follow the following notation and assumptions till the end of this section.

Let $G \subset \widehat{G}$ be connected reductive groups. Let $B \subset G$ and $\widehat{B} \subset \widehat{G}$ be Borel subgroups, and $H \subset B$ and $\widehat{H} \subset \widehat{B}$ be maximal tori. We assume that $H \subset \widehat{H}$ and there exists $x \in N(\widehat{H})$ such that $B=x \widehat{B} x^{-1} \cap G$, where $N(\widehat{H})$ is the normalizer of $\widehat{H}$ in $\widehat{G}$.

Let $\widehat{B} \subset \widehat{P} \subset \widehat{Q}$ be (standard) parabolic subgroups in $\widehat{G}$. Define the standard parabolic subgroups in $G: P=x \widehat{P} x^{-1} \cap G, Q=x \widehat{Q} x^{-1} \cap G$.

Define an embedding of the flag verieties

$$
f_{2}: G / P \hookrightarrow \widehat{G} / \widehat{P}, g P \mapsto x^{-1} g x \widehat{P}
$$

and similarly $f: G / Q \hookrightarrow \widehat{G} / \widehat{Q}$. Then, we have a commutative diagram

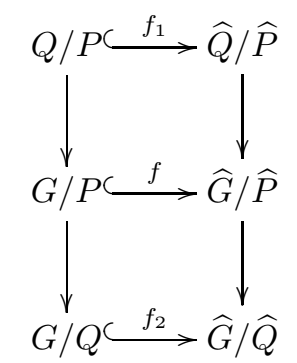

where the vertical maps are the standard maps. The Weyl group for $\widehat{G}$ is denoted by $\widehat{W}$ and similarly $W$ for $G$. Let $\widehat{w} \in \widehat{W}^{\widehat{P}}$ be such that

$$
\operatorname{dim} \widehat{G} / \widehat{P}-\ell(\widehat{w})=\operatorname{dim} G / P, \quad \text { and } \operatorname{dim} \widehat{G} / \widehat{Q}-\ell(\widehat{u})=\operatorname{dim} G / Q,
$$

where $\widehat{w}=\widehat{u} \widehat{v}$ is the unique decomposition with $\widehat{u} \in \widehat{W}^{\widehat{Q}}$ and $\widehat{v} \in \widehat{W}^{\widehat{P}} \cap \widehat{W}_{\widehat{Q}}$. Thus, we automatically get

$$
\operatorname{dim} \widehat{Q} / \widehat{P}-\ell(\widehat{v})=\operatorname{dim} Q / P .
$$

Recall from Section 5 that $\widehat{\Phi}_{\widehat{w}}^{\widehat{P}}$ is the shifted cell $\widehat{w}^{-1} \widehat{B} \widehat{w} \widehat{P} / \widehat{P} \subset \widehat{G} / \widehat{P}$. 


\section{SHRAWAN KUMAR}

Lemma 59. For any $g=q \dot{\widehat{u}}^{-1}$, with $q \in \widehat{Q}$ and a representative $\dot{\hat{u}}$ of $\widehat{u}$,

$$
g \widehat{C_{\widehat{w}}} \widehat{P} \cap \widehat{Q} / \widehat{P}=q \widehat{C}_{\widehat{v}}^{\widehat{P}} .
$$

Proof. Let $\widehat{R}_{\widehat{w}}:=\widehat{R}^{+} \cap \widehat{w}^{-1} \widehat{R}^{-}$, where $\widehat{R}^{+}$(resp. $\widehat{R}^{-}$) is the set of positive (resp. negative) roots of $\widehat{G}$. Let $\widehat{U}_{\widehat{R}_{\widehat{w}}}$ (resp. $\widehat{U}_{\widehat{R}_{\widehat{w}}}^{-}$) be the unipotent subgroup of the unipotent radical of $\widehat{B}$ (resp. $\widehat{B}^{-}$) such that its Lie algebra has roots $\widehat{R}_{\widehat{w}}$ (resp. $-\widehat{R}_{\widehat{w}}$ ). Then, it is easy to see from $\left[\mathrm{K}_{1}\right.$, Lemma 1.3.14] that

$$
\widehat{\Phi}_{\widehat{w}}^{\widehat{P}}=\widehat{v}^{-1} \widehat{U}_{\widehat{R}_{\widehat{u}}}^{-} \widehat{v} \widehat{U}_{\widehat{R}}^{-} \widehat{P} / \widehat{P} .
$$

Also, it is easy to see that

$$
\widehat{U}_{\widehat{R}_{\widehat{u}}}^{-} \cap \widehat{Q}=(1) .
$$

Thus, by the identities (75) and (76),

$$
g \widehat{C}_{\widehat{w}}^{\widehat{P}} \cap \widehat{Q} / \widehat{P}=q \widehat{v} \widehat{U}_{\widehat{R}_{\widehat{v}}}^{-} \widehat{P} / \widehat{P}=q \widehat{C}_{\widehat{v}}^{\widehat{P}} .
$$

This proves the lemma.

Definition 13. Define a subset $\mathscr{X}=\mathscr{X}_{\widehat{u}}$ by

$$
\mathscr{X}=\left\{(\bar{g}, \bar{h}) \in G / Q \times \widehat{G} / \widehat{B}: f_{2}(\bar{g}) \in h \widehat{C}_{\widehat{u}}^{\widehat{Q}}\right\},
$$

where $\widehat{C}_{\widehat{u}}^{\widehat{Q}}$ is the Schubert cell $\widehat{B} \widehat{u} \widehat{Q} / \widehat{Q}$ in $\widehat{G} / \widehat{Q}$, and $\bar{g}$ denotes $g Q$, etc.

Let $Q$ act on $\widehat{Q} \widehat{u}^{-1} \widehat{B} / \widehat{B}$ via $q \odot z=\left(x^{-1} q x\right) \cdot z$.

Lemma 60. There is an isomorphism

$$
\mu: G \times^{Q}\left(\widehat{Q} \widehat{u}^{-1} \widehat{B} / \widehat{B}\right) \stackrel{\sim}{\rightarrow} \mathscr{X}, \mu[g, z]=\left(\bar{g},\left(x^{-1} g x\right) \cdot z\right) \text { forg } \in G, z \in \widehat{Q} \widehat{u}^{-1} \widehat{B} / \widehat{B} .
$$

Thus, $\mathscr{X}$ is an irreducible smooth variety.

Proof. For $\bar{h} \in \widehat{G} / \widehat{B},(\overline{1}, \bar{h}) \in \mathscr{X} \Leftrightarrow 1 \in h \widehat{B} \widehat{u} \widehat{Q} / \widehat{Q} \Leftrightarrow h \in \widehat{Q} \widehat{u}^{-1} \widehat{B}$. Moreover, $(\bar{g}, \bar{h}) \in \mathscr{X} \Leftrightarrow\left(\overline{1}, x^{-1} g^{-1} x \bar{h}\right) \in \mathscr{X}$. From this it is easy to see that $\mu$ is an isomorphism.

Definition 14. Let $\xi_{\widehat{u}}: \widehat{Q} \widehat{u}^{-1} \widehat{B} / \widehat{B} \rightarrow \widehat{Q} / \widehat{B}$ be the map $q \widehat{u}^{-1} \widehat{B} \mapsto q \widehat{B}$, for $q \in \widehat{Q}$. This is well defined since $\left(\widehat{u}^{-1} \widehat{B} \widehat{u}\right) \cap \widehat{Q}=\left(\widehat{u}^{-1} \widehat{B} \widehat{u}\right) \cap \widehat{B}$ (and clearly $\widehat{Q}$-equivariant).

Define a subset $\mathscr{X}^{\circ} \subset \mathscr{X}$ consisting of $(\bar{g}, \bar{h}) \in \mathscr{X}$ satisfying:

(a) $\left(x^{-1} g^{-1} x h \widehat{C} \widehat{\widehat{P}}\right) \cap \widehat{Q} / \widehat{P}$ intersects $f_{1}(Q / P)$ in $\widehat{Q} / \widehat{P}$ transversally at every point of the intersection, and

(b) $\left(\xi_{\widehat{u}}\left(x^{-1} g^{-1} x \bar{h}\right) \widehat{C}_{\widehat{v}}^{\widehat{P}}\right) \cap f_{1}(Q / P)=\left(\xi_{\widehat{u}}\left(x^{-1} g^{-1} x \bar{h}\right) \widehat{X}_{\widehat{v}}^{\widehat{P}}\right) \cap f_{1}(Q / P)$.

(Recall that since $(\bar{g}, \bar{h}) \in \mathscr{X}$, we have $x^{-1} g^{-1} x h \in \widehat{Q} \widehat{u}^{-1} \widehat{B}$ by the proof of Lemma 60. Moreover, by Lemma $59,\left(x^{-1} g^{-1} x h \widehat{C}_{\widehat{w}}^{\widehat{P}}\right) \cap \widehat{Q} / \widehat{P}=\xi_{\widehat{u}}\left(x^{-1} g^{-1} x \bar{h}\right) \widehat{C} \widehat{\widehat{P}}$; in particular, it is smooth. 


\section{EIGENVALUE PROBLEM}

Definition 15. Let $Q$ act on $\widehat{Q} / \widehat{B}$ via $q \odot z=\left(x^{-1} q x\right) \cdot z$. Define $\xi: \mathscr{X} \rightarrow \mathcal{Z}:=$ $G \times{ }^{Q} \widehat{Q} / \widehat{B}$ by

$$
\xi(\mu[g, z])=\left[g, \xi_{\widehat{u}}(z)\right] \quad \text { for } \quad g \in G, z \in \widehat{Q} \widehat{u}^{-1} \widehat{B} / \widehat{B} .
$$

Proposition 61. The subset $\mathscr{X}^{\circ}$ contains a dense open subset of $\mathscr{X}$.

Proof. By Theorem 6 and the identity (74), there exists a dense open subset $V \subset$ $\widehat{Q} / \widehat{B}$, which is stable under the left multiplication by $x^{-1} Q x$, such that for any $\bar{q} \in V$

(a) $q \widehat{C}_{\widehat{V}} \widehat{P} \cap f_{1}(Q / P)$ is a transverse intersection in $\widehat{Q} / \widehat{P}$ (at any point of the intersection), and

(b) $q \widehat{C}_{\widehat{v}}^{\widehat{P}} \cap f_{1}(Q / P)=q \widehat{X}_{\widehat{v}}^{\widehat{P}} \cap f_{1}(Q / P)$.

Now, $(\bar{g}, \bar{h}) \in \mathscr{X}$ belongs to $\mathscr{X}^{\circ}$ if $\xi_{\widehat{u}}\left(x^{-1} g^{-1} x \bar{h}\right) \in V$. Thus, $\mathscr{X}^{\circ}$ contains a dense open subset of $\mathscr{X}$.

Let $\sigma: \mathscr{X} \rightarrow \widehat{G} / \widehat{B}$ be the projection on the second factor.

Lemma 62. Assume that $f_{2}^{*}\left[\widehat{X}_{\widehat{u}}^{\widehat{Q}}\right] \neq 0 \in H^{*}(G / Q)$. Then, $\sigma$ is a dominant morphism. Moreover,

$$
\operatorname{dim} \mathscr{X}=\operatorname{dim} \widehat{G} / \widehat{B} .
$$

Proof. Since codim $\widehat{C}_{\widehat{u}}^{\widehat{Q}}=\operatorname{dim} G / Q$ (by assumption $\left.(73)\right)$ and $f_{2}^{*}\left(\left[\widehat{X}_{\widehat{u}}^{\widehat{Q}}\right]\right) \neq 0$, we get that $h \widehat{C}_{\widehat{u}}^{\widehat{Q}} \cap f(G / Q)$ is a finite nonempty subset for general $h \in \widehat{G}$. Thus, the map $\sigma$ is dominant and on a dense open subset of $\widehat{G} / \widehat{B}, \sigma$ has finite fibres. Thus, $\operatorname{dim} \widehat{G} / \widehat{B}=\operatorname{dim} \mathscr{X}$.

The following result, as well as Theorem 64, is due to Richmond $\left[\mathrm{Ri}_{2}\right]$ (and also due to Ressayre $\left.\left[\mathrm{R}_{6}\right]\right)$.

Theorem 63. Let $\widehat{w} \in \widehat{W}^{\widehat{P}}$ be such that it satisfies the condition (73). Write

$$
\begin{aligned}
& f^{*}\left(\left[\widehat{X}_{\hat{w}}^{\widehat{P}}\right]\right)=d[\mathrm{pt}] \in H^{*}(G / P), \\
& f_{2}^{*}\left(\left[\widehat{X}_{\widehat{u}}^{\widehat{Q}}\right]\right)=d_{1}[\mathrm{pt}] \in H^{*}(G / Q), \\
& f_{1}^{*}\left(\left[\widehat{X}_{\hat{v}}^{\widehat{P}}\right]\right)=d_{2}[\mathrm{pt}] \in H^{*}(Q / P)
\end{aligned}
$$

for some integers $d, d_{1}, d_{2}$. Then, $d=d_{1} d_{2}$.

(Observe that $\widehat{X}_{\widehat{v}}^{\widehat{P}} \subset \widehat{Q} / \widehat{P}$ since $\widehat{v} \in \widehat{W}^{\widehat{P}} \cap \widehat{W}_{\widehat{Q}}$ ).

Proof. Assume first that $d_{1} \neq 0$. Choose general elements $y=\bar{h} \in \widehat{G} / \widehat{B}$ such that

(a) $h \widehat{C}_{\widehat{u}}^{\widehat{Q}} \cap f_{2}(G / Q)$ is a transverse intersection,

(b) $h \widehat{C}_{\widehat{u}}^{\widehat{Q}} \cap f_{2}(G / Q)=h \widehat{X}_{\widehat{u}}^{\widehat{Q}} \cap f_{2}(G / Q)$,

(c) $h \widehat{C} \widehat{\widehat{P}} \cap f(G / P)$ is a transverse intersection,

(d) $h \widehat{C}_{\widehat{w}}^{\widehat{P}} \cap f(G / P)=h \widehat{X}_{\widehat{w}}^{\widehat{P}} \cap f(G / P)$,

(e) $(\bar{g}, \bar{h}) \in \mathscr{X}^{\circ}$ for all $\bar{g} \in G / Q$ such that $(\bar{g}, \bar{h}) \in \mathscr{X}$. 


\section{SHRAWAN KUMAR}

We now show the existence of such $y$ 's. Let $\mathfrak{V}$ be an open dense subset of $\widehat{G} / \widehat{B}$ satisfying (a)-(d), which exists by Theorem 6 and the assumption (73). Take a dense open subset $\widehat{\mathscr{X}}^{o}$ of $\mathscr{X}$ contained in $\mathscr{X}^{o} \cap \sigma^{-1}(\mathfrak{V})$. Then,

$$
\operatorname{dim}\left(\overline{\sigma\left(\mathscr{X} \backslash \widehat{\mathscr{X}}^{o}\right)}\right) \leq \operatorname{dim}\left(\mathscr{X}-\widehat{\mathscr{X}}^{o}\right)<\operatorname{dim} \mathscr{X}=\operatorname{dim} \widehat{G} / \widehat{B}
$$

where the last equality follows by Lemma 62 . Thus, $\sigma^{-1}\left(\overline{\sigma\left(\mathscr{X} \backslash \widehat{\mathscr{X}^{\circ}}\right)}\right)$ is a proper closed subset of $\mathscr{X}$. Take any $y^{\prime} \in \mathscr{X} \backslash \sigma^{-1}\left(\overline{\sigma\left(\mathscr{X} \backslash \widehat{\mathscr{X}^{\circ}}\right)}\right)$. Then, clearly $y^{\prime} \in \mathscr{X}^{o}$ and $y=\sigma\left(y^{\prime}\right) \in \mathfrak{V}$. This proves the existence of $y$ satisfying (a)-(e).

For any $y=\bar{h} \in \widehat{G} / \widehat{B}$ satisfying the conditions (a)-(e),

$$
d_{1}=\left|\left(h \widehat{C}_{\widehat{u}}^{\widehat{Q}}\right) \cap f_{2}(G / Q)\right|, \quad d=\left|\left(h \widehat{C}_{\widehat{w}}^{\widehat{P}}\right) \cap f(G / P)\right| .
$$

Moreover, under the projection map $\left(h \widehat{C}_{\widehat{w}}^{\widehat{P}}\right) \cap f(G / P) \stackrel{\pi}{\rightarrow}\left(h \widehat{C}_{\widehat{u}}^{\widehat{Q}}\right) \cap f_{2}(G / Q)$, for any $\bar{g} \in f_{2}^{-1}\left(h \widehat{C}_{\widehat{u}}^{\widehat{Q}} \cap f_{2}(G / Q)\right)$,

$\pi^{-1}\left(f_{2}(\bar{g})\right) \simeq\left(x^{-1} g^{-1} x h \widehat{C}_{\widehat{w}}^{\widehat{P}} \cap \widehat{Q} / \widehat{P}\right) \cap f_{1}(Q / P)=\left(\xi_{\widehat{u}}\left(x^{-1} g^{-1} x \bar{h}\right) \widehat{C}_{\widehat{v}}^{\widehat{P}}\right) \cap f_{1}(Q / P)$,

where the last equality follows from Definition 14 and the condition (e). The last intersection is a transverse intersection in $\widehat{Q} / \widehat{P}$ and

$$
\left(\xi_{\widehat{u}}\left(x^{-1} g^{-1} x \bar{h}\right) \widehat{X}_{\widehat{v}}^{\widehat{P}}\right) \cap f_{1}(Q / P)=\left(\xi_{\widehat{u}}\left(x^{-1} g^{-1} x \bar{h}\right) \widehat{C}_{\widehat{v}}^{\widehat{P}}\right) \cap f_{1}(Q / P),
$$

by the definition of $\mathscr{X}^{\circ}$. Thus,

$$
\left|\pi^{-1}\left(f_{2}(\bar{g})\right)\right|=d_{2} .
$$

This gives $d=d_{1} d_{2}$, proving the theorem for the case $d_{1} \neq 0$.

We finally show that if $d_{1}=0$, then $d=0$. For, if not, take general $g \in \widehat{G}$ such that $g \widehat{C}_{\widehat{w}}^{\widehat{P}} \cap f(G / P)$ is nonempty. Then, $g \widehat{C}_{\widehat{u}}^{\widehat{Q}} \cap f(G / Q)$ is nonempty too since $\pi\left(g \widehat{C}_{\widehat{w}}^{\widehat{P}} \cap f(G / P)\right) \subset g \widehat{C}_{\widehat{u}}^{\widehat{Q}} \cap f(G / Q)$. This proves the theorem completely.

A particular case of Definition 8 is the following.

Definition 16. Let $\widehat{w} \in \widehat{W}^{\widehat{P}}$ be such that $\operatorname{codim} \widehat{\Phi} \widehat{\widehat{P}}=\operatorname{dim} G / P$. Then, $\widehat{\Phi}_{\widehat{w}}^{\widehat{P}}$ is said to be L-movable for the embedding $f: G / P \rightarrow \widehat{G} / \widehat{P}$ if

$$
T_{e}(G / P) \stackrel{(d f)_{e}}{\longrightarrow} \frac{T_{\widehat{e}}(\widehat{G} / \widehat{P})}{T_{\widehat{e}}\left(\widehat{l} \widehat{\Phi}_{\widehat{w}}^{\widehat{P}}\right)}
$$

is an isomorphism for some $\widehat{l} \in \widehat{L}_{\widehat{P}}$, where $\widehat{L}_{\widehat{P}}$ is the Levi subgroup of $\widehat{P}$ containing $\widehat{H}$.

Recall the definition of the elements $x_{i} \in \mathfrak{h}$ from the equation (7). 
Theorem 64. Let $\widehat{w} \in \widehat{W}^{\widehat{P}}$ be such that $\widehat{\Phi} \widehat{\widehat{P}} \subset \widehat{G} / \widehat{P}$ is L-movable for the embedding $f: G / P \rightarrow \widehat{G} / \widehat{P}$ (in particular, codim $\widehat{\Phi} \widehat{\widehat{P}}=\operatorname{dim} G / P)$. Assume further that there exists a dominant regular element $y_{o} \in \mathfrak{z}\left(L_{Q}\right)$ (i.e., an element of the form $\left.y_{o}=\sum_{\alpha_{i} \in \Delta(G) \backslash \Delta(Q)} r_{i} x_{i}, \quad r_{i}>0\right)$ such that $\operatorname{Ad}\left(x^{-1}\right) \cdot y_{o} \in \mathfrak{z}\left(\widehat{L}_{\widehat{Q}}\right)$, where $\mathfrak{z}\left(L_{Q}\right)$ denotes the Lie algebra of the center of the Levi subgroup $L_{Q}$ of $Q$. Then, $\widehat{\Phi} \widehat{\widehat{u}} \subset \widehat{G} / \widehat{Q}$ is Levi movable for the embedding $f_{2}: G / Q \rightarrow \widehat{G} / \widehat{Q}$ and $\widehat{\Phi} \widehat{\widehat{V}} \subset \widehat{Q} / \widehat{P}$ is Levi movable for the embedding $f_{1}: Q / P \rightarrow \widehat{Q} / \widehat{P}$.

In particular, the assumption (and hence the conclusion) of Theorem 63 is automatically satisfied under the above assumptions.

Proof. Let $C$ be the group $x Z\left(\widehat{L}_{\widehat{Q}}\right) x^{-1} \cap H$, where $Z\left(\widehat{L}_{\widehat{Q}}\right)$ is the center of the Levi subgroup $\widehat{L}_{\widehat{Q}}$ of $\widehat{Q}$. Observe that $C \subset Z\left(L_{Q}\right)$.

Take $\widehat{l} \in \widehat{L}_{\widehat{P}}$ such that the map $\varphi$ in the following big diagram is an isomorphism, which is possible since $\widehat{\Phi}_{\widehat{w}}^{\widehat{P}}$ is $L$-movable for the embedding $f$. Define an action of $C$ on $\widehat{G} / \widehat{P}$ via $t \odot y=\left(x^{-1} t x\right) y$, for $y \in \widehat{G} / \widehat{P}$ and $t \in C$. The left multiplication map $\widehat{G} / \widehat{P} \rightarrow \widehat{G} / \widehat{P}, y \mapsto \widehat{l} y$ commutes with the action of $C$ and hence we have a $C$-module isomorphism

$$
\frac{T_{\widehat{e}}(\widehat{G} / \widehat{P})}{T_{\widehat{e}}\left(\widehat{\Phi}_{\widehat{w}} \widehat{P}\right)} \stackrel{\sim}{\rightarrow} \frac{T_{\widehat{e}}(\widehat{G} / \widehat{P})}{T_{\widehat{e}}\left(\widehat{l} \widehat{l}_{\widehat{w}}^{\widehat{P}}\right)} .
$$

We have the following commutative diagram, where the maps $\varphi_{1}, \varphi, \varphi_{2}$ are induced by the embeddings $f_{1}, f, f_{2}$ respectively. (By $\left[\mathrm{K}_{1}\right.$, Lemma 1.3.14], it is easy to see that $\widehat{\Phi} \widehat{\widehat{P}} \subset \widehat{\Phi}_{\widehat{\omega}}^{\widehat{P}}$ and $\widehat{\Phi}_{\widehat{w}}^{\widehat{P}}$ maps to $\widehat{v}^{-1} \widehat{\Phi}_{\widehat{u}}^{\widehat{Q}}$ under the projection $\widehat{G} / \widehat{P} \rightarrow \widehat{G} / \widehat{Q}$.) Moreover, in the following diagram, all the modules are $C$-modules and all the maps are $C$-module maps, where the action of $C$ on the left vertical side of the diagram is induced from the action of $C$ on $G / P$ via the left multiplication:

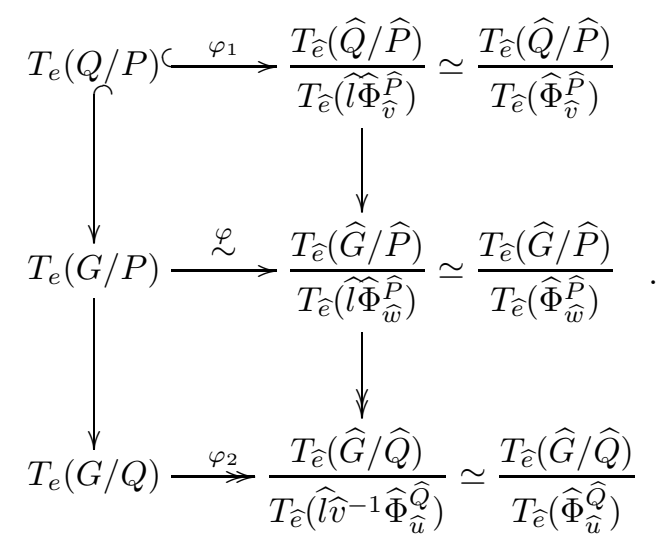

By the identity (30), the sum of the set of roots in $T_{\widehat{e}}(\widehat{G} / \widehat{P})=-\chi_{1}^{\widehat{P}}$ and the sum of the set of roots in $T_{\widehat{e}}(\widehat{G} / \widehat{P}) / T_{\widehat{e}}\left(\widehat{\Phi}_{\widehat{w}}^{\widehat{P}}\right)=-\chi_{\widehat{w}}$. Thus, from the isomorphism $\varphi$, we get:

$$
-\chi_{1 \mid \mathfrak{c}}^{P}=-\widehat{\chi}_{\widehat{w}_{\mid c}}^{\widehat{P}},
$$




\section{SHRAWAN KUMAR}

where $\widehat{\chi}_{\widehat{w} \mid \mathfrak{c}}^{\widehat{P}}$ refers to the twisted action $\odot$, i.e., $\widehat{\chi}_{\widehat{w}}^{\widehat{P}}(y)=\chi_{\widehat{w}}^{\widehat{P}}\left(\operatorname{Ad} x^{-1} \cdot y\right)$, for $y \in \mathfrak{c}$, where $\mathfrak{c}:=$ Lie $C$.

Let $M$ be the kernel of $\varphi_{2}$ and let $\beta$ be the sum of roots in $M$. Then, from the surjective map $\varphi_{2}$, we get

$$
-\chi_{1}^{Q}-\beta=-\widehat{\chi}_{\widehat{u}}^{\widehat{Q}} \quad \text { restricted to } \quad \mathfrak{c}
$$

But, it is easy to see that

$$
\chi_{1 \mid \mathfrak{c}}^{P}=\chi_{1 \mid \mathfrak{c}}^{Q}, \quad \text { and } \widehat{\chi}_{\widehat{w} \mid \mathfrak{c}}^{\widehat{P}}=\widehat{\chi}_{\widehat{u} \mid \mathfrak{c}}^{\widehat{Q}} .
$$

Thus, combining the equations (78)-(80), we get $\beta_{\mid \mathfrak{c}} \equiv 0$. In particular, $\beta\left(y_{0}\right)=0$. But since $y_{o}$ is a dominant regular element of $\mathfrak{z}\left(L_{Q}\right)$ and $\beta$ is a positive sum of roots in $R_{G}^{-} \backslash R_{L_{Q}}^{-}$, this is possible only if $\beta=0$, i.e., $M$ is zero-dimensional. This shows that $M=0$ and hence $\varphi_{2}$ is an isomorphism. Since $\varphi_{1}$ is injective, by dimensional consideration, $\varphi_{1}$ is an isomorphism as well. This proves the theorem.

Remark 15. As in [ReR, Lemma 3.4], by virtue of Theorem 64, the multiplicative formula for the decomposition of structure constants as in Theorem 63 applies to all the structure constants associated to the homomorphism $\theta_{0}^{\delta}$ of Theorem 58 .

Specializing Theorems 63 and 64 to the diagonal case, we immediately get the following. This result was obtained by Richmond for type A flag varieties in $\left[\mathrm{Ri}_{1}\right.$, Thm. 3], for type $\mathrm{C}$ flag varieties in his $\mathrm{PhD}$ thesis, and in general in $\left[\mathrm{Ri}_{2}\right]$.

Corollary 65. Let $G, B, H$ be as in Section 2; in particular, $G$ is a connected semisimple group. Let $B \subset P \subset Q$ be (standard) parabolic subgroups. Let $\left\{w_{j}\right\}_{1 \leq j \leq s} \subset W^{P}$ be such that $\sum_{j=1}^{s} \operatorname{codim} X_{w_{j}}^{P}=\operatorname{dim} G / P$. Assume further that

$$
\sum_{j=1}^{s} \operatorname{codim} X_{u_{j}}^{Q}=\operatorname{dim} G / Q
$$

(and hence $\left.\sum_{j=1}^{s} \operatorname{codim}_{Q / P} X_{v_{j}}^{P}=\operatorname{dim} Q / P\right)$, where $w_{j}=u_{j} v_{j}$ is the unique decomposition with $u_{j} \in W^{Q}$ and $v_{j} \in W^{P} \cap W_{Q}$. Write

$$
\begin{aligned}
{\left[X_{w_{1}}^{P}\right] \ldots\left[X_{w_{s}}^{P}\right] } & =d[\mathrm{pt}] \in H^{*}(G / P), \\
{\left[X_{u_{1}}^{Q}\right] \ldots\left[X_{u_{s}}^{Q}\right] } & =d_{1}[\mathrm{pt}] \in H^{*}(G / Q), \text { and } \\
{\left[X_{v_{1}}^{P}\right] \ldots\left[X_{v_{s}}^{P}\right] } & =d_{2}[\mathrm{pt}] \in H^{*}(Q / P) .
\end{aligned}
$$

Then, $d=d_{1} d_{2}$.

If $\left\{w_{j}\right\}$ is $L_{P}$-movable for $G / P$, then $\left\{u_{j}\right\}$ (resp. $\left\{v_{j}\right\}$ ) is $L_{Q}$-movable for $G / Q$ (resp. $L_{P}$-movable for $Q / P$ ). In particular, $(81)$ is automatically satisfied.

Remark 16. Knutson-Purbhoo [KP] have shown that for a $(d-1)$-step flag variety $G / P$ (for $G=\mathrm{SL}(n)$ ), any structure constant for the deformed product $\odot_{0}$ is a product of $d(d-1) / 2$ Littlewood-Richardson numbers. This refines the factorization into $d-1$ factors as in Corollary 65 . 


\section{Tables of the deformed product $\odot$ for the groups of type $B_{2}, G_{2}, B_{3}$, and $C_{3}$}

We give below the multiplication tables under the deformed product $\odot$ for $G / P$ for the complex simple groups of type $B_{2}, G_{2}, B_{3}$, and $C_{3}$, and maximal parabolic subgroups $P$. Since we are only considering maximal parabolics, we have only one indeterminate, which we denote by $\tau$. We let $r, s$ and $t$ be the simple reflections of any group of rank 3 (and $r, s$ for the simple groups of rank 2) associated to the nodes from left to right of the Dynkin diagram following the Bourbaki [Bo, Planche I-IX] convention (so $t$ corresponds to the long simple root in the case of $C_{3}$ and the short simple root in the case of $B_{3}$ ).

These tables for rank 3 groups are taken from [BK $1, \S 10]$ and [KuLM, $\S 4]$.

Example 1. $G=\mathrm{B}_{2}, P=P_{1}$ : In the following $b_{1}=\epsilon_{r}^{P}, b_{2}=\epsilon_{s r}^{P}, b_{3}=\epsilon_{r s r}^{P}$.

\begin{tabular}{|c|c|c|c|}
\hline$H^{*}\left(G / P_{1}\right)$ & $b_{1}$ & $b_{2}$ & $b_{3}$ \\
\hline$b_{1}$ & $2 b_{2}$ & $b_{3}$ & 0 \\
\hline
\end{tabular}

Example 2. $G=\mathrm{B}_{2}, P=P_{2}$ : In the following $b_{1}=\epsilon_{s}^{P}, b_{2}=\epsilon_{r s}^{P}, b_{3}=\epsilon_{s r s}^{P}$.

\begin{tabular}{|c|c|c|c|}
\hline$H^{*}\left(G / P_{2}\right)$ & $b_{1}$ & $b_{2}$ & $b_{3}$ \\
\hline$b_{1}$ & $\tau b_{2}$ & $b_{3}$ & 0 \\
\hline
\end{tabular}

Example 3. $G=\mathrm{G}_{2}, P=P_{1}$ : In the following $a_{1}=\epsilon_{r}^{P}, a_{2}=\epsilon_{s r}^{P}, a_{3}=\epsilon_{r s r}^{P}, a_{4}=$ $\epsilon_{s r s r}^{P}, a_{5}=\epsilon_{r s r s r}^{P}$.

\begin{tabular}{|c|c|c|c|c|c|}
\hline$H^{*}\left(G / P_{1}\right)$ & $a_{1}$ & $a_{2}$ & $a_{3}$ & $a_{4}$ & $a_{5}$ \\
\hline$a_{1}$ & $\tau^{2} a_{2}$ & $5 \tau a_{3}$ & $\tau^{2} a_{4}$ & $a_{5}$ & 0 \\
\hline$a_{2}$ & & $5 \tau a_{4}$ & $a_{5}$ & 0 & 0 \\
\hline
\end{tabular}

Example 4. $G=\mathrm{G}_{2}, P=P_{2}$ : In the following $a_{1}=\epsilon_{s}^{P}, a_{2}=\epsilon_{r s}^{P}, a_{3}=\epsilon_{s r s}^{P}, a_{4}=$ $\epsilon_{r s r s}^{P}, a_{5}=\epsilon_{s r s r s}^{P}$.

\begin{tabular}{|c|c|c|c|c|c|}
\hline$H^{*}\left(G / P_{2}\right)$ & $a_{1}$ & $a_{2}$ & $a_{3}$ & $a_{4}$ & $a_{5}$ \\
\hline$a_{1}$ & $3 a_{2}$ & $2 \tau a_{3}$ & $3 a_{4}$ & $a_{5}$ & 0 \\
\hline$a_{2}$ & & $2 \tau a_{4}$ & $a_{5}$ & 0 & 0 \\
\hline
\end{tabular}

Example 5. $G=\mathrm{B}_{3}, P=P_{1}$ : In the following $b_{1}=\epsilon_{r}^{P}, b_{2}=\epsilon_{s r}^{P}, b_{3}=\epsilon_{t s r}^{P}, b_{4}=$ $\epsilon_{s t s r}^{P}, b_{5}=\epsilon_{r s t s r}^{P}$.

\begin{tabular}{|c|c|c|c|c|c|}
\hline$H^{*}\left(G / P_{1}\right)$ & $b_{1}$ & $b_{2}$ & $b_{3}$ & $b_{4}$ & $b_{5}$ \\
\hline$b_{1}$ & $b_{2}$ & $2 b_{3}$ & $b_{4}$ & $b_{5}$ & 0 \\
\hline$b_{2}$ & & $2 b_{4}$ & $b_{5}$ & 0 & 0 \\
\hline
\end{tabular}

Example 6. $G=\mathrm{B}_{3}, P=P_{2}$ : In the following $b_{1}=\epsilon_{s}^{P}, b_{2}^{\prime}=\epsilon_{r s}^{P}, b_{2}^{\prime \prime}=\epsilon_{t s}^{P}, b_{3}^{\prime}=$ $\epsilon_{r t t}^{P}, b_{3}^{\prime \prime}=\epsilon_{\text {sts }}^{P}, b_{4}^{\prime}=\epsilon_{\text {srts }}^{P}, b_{4}^{\prime \prime}=\epsilon_{\text {rsts }}^{P}, b_{5}^{\prime}=\epsilon_{\text {tsrts }}^{P}, b_{5}^{\prime \prime}=\epsilon_{r s t r s}^{P}, b_{6}=\epsilon_{r t s r t s}^{P}, b_{7}=$
$\epsilon_{\text {srtsrts. }}^{P}$.

\begin{tabular}{|c|c|c|c|c|c|}
\hline$H^{*}\left(G / P_{2}\right)$ & $b_{1}$ & $b_{2}^{\prime}$ & $b_{2}^{\prime \prime}$ & $b_{3}^{\prime}$ & $b_{3}^{\prime \prime}$ \\
\hline$b_{1}$ & $b_{2}^{\prime}+2 b_{2}^{\prime \prime}$ & $2 b_{3}^{\prime}$ & $b_{3}^{\prime}+b_{3}^{\prime \prime}$ & $2 \tau b_{4}^{\prime}+\tau b_{4}^{\prime \prime}$ & $\tau b_{4}^{\prime}+2 \tau b_{4}^{\prime \prime}$ \\
\hline$b_{2}^{\prime}$ & & $2 \tau b_{4}^{\prime}$ & $\tau b_{4}^{\prime}+\tau b_{4}^{\prime \prime}$ & $2 \tau b_{5}^{\prime}+\tau b_{5}^{\prime \prime}$ & $\tau b_{5}^{\prime \prime}$ \\
\hline$b_{2}^{\prime \prime}$ & & & $\tau b_{4}^{\prime}+\tau b_{4}^{\prime \prime}$ & $\tau b_{5}^{\prime}+\tau b_{5}^{\prime \prime}$ & $\tau b_{5}^{\prime}+\tau b_{5}^{\prime \prime}$ \\
\hline$b_{3}^{\prime}$ & & & & $2 \tau b_{6}$ & $\tau b_{6}$ \\
\hline$b_{3}^{\prime \prime}$ & & & & & $2 \tau b_{6}$ \\
\hline
\end{tabular}


SHRAWAN KUMAR

\begin{tabular}{|c|c|c|c|c|c|c|}
\hline$H^{*}\left(G / P_{2}\right)$ & $b_{4}^{\prime}$ & $b_{4}^{\prime \prime}$ & $b_{5}^{\prime}$ & $b_{5}^{\prime \prime}$ & $b_{6}$ & $b_{7}$ \\
\hline$b_{1}$ & $2 b_{5}^{\prime}+b_{5}^{\prime \prime}$ & $b_{5}^{\prime \prime}$ & $b_{6}$ & $2 b_{6}$ & $b_{7}$ & 0 \\
\hline$b_{2}^{\prime}$ & $2 b_{6}$ & 0 & $b_{7}$ & 0 & 0 & 0 \\
\hline$b_{2}^{\prime \prime}$ & $b_{6}$ & $b_{6}$ & 0 & $b_{7}$ & 0 & 0 \\
\hline$b_{3}^{\prime}$ & $b_{7}$ & 0 & 0 & 0 & 0 & 0 \\
\hline$b_{3}^{\prime \prime}$ & 0 & $b_{7}$ & 0 & 0 & 0 & 0 \\
\hline
\end{tabular}

Example 7. $G=\mathrm{B}_{3}, P=P_{3}$ : In the following $b_{1}=\epsilon_{t}^{P}, b_{2}=\epsilon_{s t}^{P}, b_{3}^{\prime}=\epsilon_{r s t}^{P}, b_{3}^{\prime \prime}=$ $\epsilon_{t s t}^{P}, b_{4}=\epsilon_{t r s t}^{P}, b_{5}=\epsilon_{s t r s t}^{P}, b_{6}=\epsilon_{\text {tstrst }}^{P}$.

\begin{tabular}{|c|c|c|c|c|c|c|c|}
\hline$H^{*}\left(G / P_{3}\right)$ & $b_{1}$ & $b_{2}$ & $b_{3}^{\prime}$ & $b_{3}^{\prime \prime}$ & $b_{4}$ & $b_{5}$ & $b_{6}$ \\
\hline$b_{1}$ & $\tau b_{2}$ & $\tau b_{3}^{\prime}+b_{3}^{\prime \prime}$ & $b_{4}$ & $\tau b_{4}$ & $\tau b_{5}$ & $b_{6}$ & 0 \\
\hline$b_{2}$ & & $2 b_{4}$ & $b_{5}$ & $\tau b_{5}$ & $b_{6}$ & 0 & 0 \\
\hline$b_{3}^{\prime}$ & & & 0 & $b_{6}$ & 0 & 0 & 0 \\
\hline$b_{3}^{\prime \prime}$ & & & & 0 & 0 & 0 & 0 \\
\hline
\end{tabular}

Example 8. $G=\mathrm{C}_{3}, P=P_{1}$ : In the following $a_{1}=\epsilon_{r}^{P}, a_{2}=\epsilon_{s r}^{P}, a_{3}=\epsilon_{t s r}^{P}, a_{4}=$ $\epsilon_{\text {stsr }}^{P}, a_{5}=\epsilon_{r s t s r}^{P}$.

\begin{tabular}{|c|c|c|c|c|c|}
\hline$H^{*}\left(G / P_{1}\right)$ & $a_{1}$ & $a_{2}$ & $a_{3}$ & $a_{4}$ & $a_{5}$ \\
\hline$a_{1}$ & $a_{2}$ & $\tau a_{3}$ & $a_{4}$ & $a_{5}$ & 0 \\
\hline$a_{2}$ & & $\tau a_{4}$ & $a_{5}$ & 0 & 0 \\
\hline
\end{tabular}

Example 9. $G=C_{3}, P=P_{2}$ : In the following $a_{1}=\epsilon_{s}^{P}, a_{2}^{\prime}=\epsilon_{r s}^{P}, a_{2}^{\prime \prime}=\epsilon_{t s}^{P}, a_{3}^{\prime}=$ $\epsilon_{r t s}^{P}, a_{3}^{\prime \prime}=\epsilon_{s t s}^{P}, a_{4}^{\prime}=\epsilon_{s r t s}^{P}, a_{4}^{\prime \prime}=\epsilon_{r s t s}^{P}, a_{5}^{\prime}=\epsilon_{t s r t s}^{P}, a_{5}^{\prime \prime}=\epsilon_{r s t r s}^{P}, a_{6}=\epsilon_{r t s r t s}^{P}, a_{7}=$ $\epsilon_{\text {srtsrts }}^{P}$.

\begin{tabular}{|c|c|c|c|c|c|}
\hline$H^{*}\left(G / P_{2}\right)$ & $a_{1}$ & $a_{2}^{\prime}$ & $a_{2}^{\prime \prime}$ & $a_{3}^{\prime}$ & $a_{3}^{\prime \prime}$ \\
\hline$a_{1}$ & $a_{2}^{\prime}+\tau a_{2}^{\prime \prime}$ & $\tau a_{3}^{\prime}$ & $a_{3}^{\prime}+a_{3}^{\prime \prime}$ & $2 \tau a_{4}^{\prime}+\tau a_{4}^{\prime \prime}$ & $\tau a_{4}^{\prime}+2 \tau a_{4}^{\prime \prime}$ \\
\hline$a_{2}^{\prime}$ & & $\tau^{2} a_{4}^{\prime}$ & $\tau a_{4}^{\prime}+\tau a_{4}^{\prime \prime}$ & $\tau^{2} a_{5}^{\prime}+\tau a_{5}^{\prime \prime}$ & $\tau a_{5}^{\prime \prime}$ \\
\hline$a_{2}^{\prime \prime}$ & & & $2 a_{4}^{\prime}+2 a_{4}^{\prime \prime}$ & $\tau a_{5}^{\prime}+2 a_{5}^{\prime \prime}$ & $\tau a_{5}^{\prime}+2 a_{5}^{\prime \prime}$ \\
\hline$a_{3}^{\prime}$ & & & & $2 \tau a_{6}$ & $\tau a_{6}$ \\
\hline$a_{3}^{\prime \prime}$ & & & & & $2 \tau a_{6}$ \\
\hline
\end{tabular}

\begin{tabular}{|c|c|c|c|c|c|c|}
\hline$H^{*}\left(G / P_{2}\right)$ & $a_{4}^{\prime}$ & $a_{4}^{\prime \prime}$ & $a_{5}^{\prime}$ & $a_{5}^{\prime \prime}$ & $a_{6}$ & $a_{7}$ \\
\hline$a_{1}$ & $\tau a_{5}^{\prime}+a_{5}^{\prime \prime}$ & $a_{5}^{\prime \prime}$ & $a_{6}$ & $\tau a_{6}$ & $a_{7}$ & 0 \\
\hline$a_{2}^{\prime}$ & $\tau a_{6}$ & 0 & $a_{7}$ & 0 & 0 & 0 \\
\hline$a_{2}^{\prime \prime}$ & $a_{6}$ & $a_{6}$ & 0 & $a_{7}$ & 0 & 0 \\
\hline$a_{3}^{\prime}$ & $a_{7}$ & 0 & 0 & 0 & 0 & 0 \\
\hline$a_{3}^{\prime \prime}$ & 0 & $a_{7}$ & 0 & 0 & 0 & 0 \\
\hline
\end{tabular}

Example 10. $G=\mathrm{C}_{3}, P=P_{3}$ : In the following $a_{1}=\epsilon_{t}^{P}, a_{2}=\epsilon_{s t}^{P}, a_{3}^{\prime}=\epsilon_{r s t}^{P}, a_{3}^{\prime \prime}=$ $\epsilon_{t s t}^{P}, a_{4}=\epsilon_{\text {trst }}^{P}, a_{5}=\epsilon_{\text {strst }}^{P}, a_{6}=\epsilon_{\text {tstrst }}^{P}$.

\begin{tabular}{|c|c|c|c|c|c|c|c|}
\hline$H^{*}\left(G / P_{3}\right)$ & $a_{1}$ & $a_{2}$ & $a_{3}^{\prime}$ & $a_{3}^{\prime \prime}$ & $a_{4}$ & $a_{5}$ & $a_{6}$ \\
\hline$a_{1}$ & $2 a_{2}$ & $2 a_{3}^{\prime}+a_{3}^{\prime \prime}$ & $a_{4}$ & $2 a_{4}$ & $2 a_{5}$ & $a_{6}$ & 0 \\
\hline$a_{2}$ & & $2 a_{4}$ & $a_{5}$ & $2 a_{5}$ & $a_{6}$ & 0 & 0 \\
\hline$a_{3}^{\prime}$ & & & 0 & $a_{6}$ & 0 & 0 & 0 \\
\hline$a_{3}^{\prime \prime}$ & & & & 0 & 0 & 0 & 0 \\
\hline
\end{tabular}


Remark 17. (a) The deformed product $\odot_{0}$ for $\mathrm{SO}(8) / P$ for all the maximal parabolic subgroups $P$ of $\mathrm{SO}(8)$ is determined in $[\mathrm{KKM}]$.

(b) The deformed product $\odot_{0}$ for $\mathrm{F}_{4} / P$ and $\mathrm{E}_{6} / P$ for all the maximal parabolic subgroups $P$ is determined by B. Lee (cf. [Le])

\section{An explicit determination of the eigencone for the ranks 2 and 3 simple Lie algebras}

The results in this section are taken from $\left[\mathrm{KLM}_{1}, \S 7\right]$ for the rank 2 root systems and $[\mathrm{KuLM}]$ for the rank 3 root systems.

In this section we describe the irredundant set of inequalities, given by Corollary 29 , determining the eigencone $\bar{\Gamma}_{3}$ inside $\mathfrak{h}_{+}^{3}$ for the ranks 2 and 3 root systems $A_{2}$, $B_{2}, G_{2}, A_{3}, B_{3}$, and $C_{3}$. Thus the following inequalities correspond to the facets of $\bar{\Gamma}_{3}$ which intersect the interior of $\mathfrak{h}_{+}^{3}$. In each of the three rank 2 (resp. rank 3 ) cases, there are 2 (resp. 3) standard maximal parabolics, hence the system breaks up into two (resp. three) subsystems.

\subsection{The inequalities for $A_{2}$}

The Weyl chamber $\mathfrak{h}_{+}$is given by

$$
\mathfrak{h}_{+}=\{(x, y, z): x+y+z=0, x \geq y \geq z\} .
$$

We give below the inequalities in terms of the triples $\left(v_{1}, v_{2}, v_{3}\right) \in \mathfrak{h}_{+}^{3}$ with $v_{j}=\left(x_{j}, y_{j}, z_{j}\right), j=1,2,3$. We only give a set of inequalities up to the action of $S_{3}$. Thus, to get the full set of inequalities, we need to symmetrize these with respect to the action of $S_{3}$ diagonally permuting the variables $x_{1}, x_{2}, x_{3} ; y_{1}, y_{2}, y_{3} ; z_{1}, z_{2}, z_{3}$.

The subsystem associated to $H^{*}\left(G / P_{1}\right)$ (before symmetrization).

$$
\begin{aligned}
& x_{1}+z_{2}+z_{3} \leq 0, \\
& y_{1}+y_{2}+z_{3} \leq 0 .
\end{aligned}
$$

These constitute 6 inequalities after symmetrization.

The subsystem associated to $H^{*}\left(G / P_{2}\right)$ (before symmetrization).

$$
\begin{aligned}
& z_{1}+x_{2}+x_{3} \geq 0, \\
& y_{1}+y_{2}+x_{3} \geq 0 .
\end{aligned}
$$

These constitute 6 inequalities after symmetrization.

To summarize, for $A_{2}$, these provide an irredundant set of altogether 12 inequalities.

\subsection{The inequalities for $B_{2}$}

The Weyl chamber $\mathfrak{h}_{+}$is given by the pairs $(x, y)$ of real numbers satisfying $x \geq$ $y \geq 0$.

The inequalities will now be in terms of $\left(v_{1}, v_{2}, v_{3}\right) \in \mathfrak{h}_{+}^{3}$ with $v_{j}=\left(x_{j}, y_{j}\right), j=$ $1,2,3$. We will need to symmetrize the inequalities with respect to the action of $S_{3}$ diagonally permuting the variables $x_{1}, x_{2}, x_{3} ; y_{1}, y_{2}, y_{3}$. 


\section{SHRAWAN KUMAR}

The subsystem associated to $H^{*}\left(G / P_{1}\right)$ (before symmetrization).

$$
\begin{aligned}
& x_{1} \leq x_{2}+x_{3}, \\
& y_{1} \leq y_{2}+x_{3} .
\end{aligned}
$$

After symmetrizing, we get 9 inequalities.

The subsystem associated to $H^{*}\left(G / P_{2}\right)$ (before symmetrization).

$$
\begin{aligned}
& x_{1}+y_{1} \leq x_{2}+y_{2}+x_{3}+y_{3}, \\
& x_{1}+y_{2} \leq y_{1}+x_{2}+x_{3}+y_{3} .
\end{aligned}
$$

After symmetrizing, we get 9 inequalities.

To summarize, for $\mathrm{B}_{2}$, these provide an irredundant set of altogether 18 inequalities.

\subsection{The inequalities for $G_{2}$}

The Weyl chamber $\mathfrak{h}_{+}$is given by the pairs $(x, y)$ of real numbers satisfying $x \geq$ $0, y \geq 0$.

The inequalities will now be in terms of $\left(v_{1}, v_{2}, v_{3}\right) \in \mathfrak{h}_{+}^{3}$ with $v_{j}=\left(x_{j}, y_{j}\right), j=$ $1,2,3$. We will need to symmetrize the inequalities with respect to the action of $S_{3}$ diagonally permuting the variables $x_{1}, x_{2}, x_{3} ; y_{1}, y_{2}, y_{3}$.

The subsystem associated to $H^{*}\left(G / P_{1}\right)$ (before symmetrization).

$$
\begin{aligned}
2 x_{1}+y_{1} & \leq 2 x_{2}+y_{2}+2 x_{3}+y_{3}, \\
x_{1}+y_{1} & \leq x_{2}+y_{2}+2 x_{3}+y_{3}, \\
x_{1} & \leq x_{2}+2 x_{3}+y_{3} .
\end{aligned}
$$

After symmetrizing, we get 15 inequalities.

The subsystem associated to $H^{*}\left(G / P_{2}\right)$ (before symmetrization).

$$
\begin{aligned}
3 x_{1}+2 y_{1} & \leq 3 x_{2}+2 y_{2}+3 x_{3}+2 y_{3}, \\
3 x_{1}+y_{1} & \leq 3 x_{2}+y_{2}+3 x_{3}+2 y_{3} \\
y_{1} & \leq y_{2}+3 x_{3}+2 y_{3} .
\end{aligned}
$$

After symmetrizing, we get 15 inequalities.

To summarize, for $\mathrm{G}_{2}$, these provide an irredundant set of altogether 30 inequalities.

\subsection{The inequalities for $A_{3}$}

The Weyl chamber $\mathfrak{h}_{+}$is given by

$$
\mathfrak{h}_{+}=\{(x, y, z, w): x+y+z+w=0, x \geq y \geq z \geq w\} .
$$

We give below the inequalities in terms of the triples $\left(v_{1}, v_{2}, v_{3}\right) \in \mathfrak{h}_{+}^{3}$ with $v_{j}=\left(x_{j}, y_{j}, z_{j}, w_{j}\right), j=1,2,3$. We only give a set of inequalities up to the action of $S_{3}$. Thus, to get the full set of inequalities, we need to symmetrize these with respect to the action of $S_{3}$ diagonally permuting the variables $x_{1}, x_{2}, x_{3} ; y_{1}, y_{2}$, $y_{3} ; z_{1}, z_{2}, z_{3} ; w_{1}, w_{2}, w_{3}$. 
EIGENVALUE PROBLEM

The subsystem associated to $H^{*}\left(G / P_{1}\right)$ (before symmetrization).

$$
\begin{array}{r}
x_{1}+w_{2}+w_{3} \leq 0 \\
y_{1}+z_{2}+w_{3} \leq 0 \\
z_{1}+z_{2}+z_{3} \leq 0
\end{array}
$$

These constitute 10 inequalities after symmetrization.

The subsystem associated to $H^{*}\left(G / P_{2}\right)$ (before symmetrization).

$$
\begin{array}{r}
x_{1}+y_{1}+z_{2}+w_{2}+z_{3}+w_{3} \leq 0, \\
x_{1}+z_{1}+y_{2}+w_{2}+z_{3}+w_{3} \leq 0, \\
x_{1}+w_{1}+x_{2}+w_{2}+z_{3}+w_{3} \leq 0, \\
y_{1}+z_{1}+y_{2}+z_{2}+z_{3}+w_{3} \leq 0, \\
x_{1}+w_{1}+y_{2}+w_{2}+y_{3}+w_{3} \leq 0, \\
y_{1}+z_{1}+y_{2}+w_{2}+y_{3}+w_{3} \leq 0 .
\end{array}
$$

These constitute 21 inequalities after symmetrization.

The subsystem associated to $H^{*}\left(G / P_{3}\right)$ (before symmetrization).

$$
\begin{array}{r}
x_{1}+y_{1}+z_{1}+y_{2}+z_{2}+w_{2}+y_{3}+z_{3}+w_{3} \leq 0, \\
x_{1}+y_{1}+w_{1}+x_{2}+z_{2}+w_{2}+y_{3}+z_{3}+w_{3} \leq 0, \\
x_{1}+z_{1}+w_{1}+x_{2}+z_{2}+w_{2}+x_{3}+z_{3}+w_{3} \leq 0 .
\end{array}
$$

These constitute 10 inequalities after symmetrization.

These 41 inequalities form an irredundant set to define $\bar{\Gamma}_{3}\left(A_{3}\right)$ inside $\mathfrak{h}_{+}^{3}$.

\subsection{The inequalities for $B_{3}$}

The Weyl chamber $\mathfrak{h}_{+}$is given by the triples $(x, y, z)$ of real numbers satisfying $x \geq y \geq z \geq 0$.

The inequalities will now be in terms of $\left(v_{1}, v_{2}, v_{3}\right) \in \mathfrak{h}_{+}^{3}$ with $v_{j}=\left(x_{j}, y_{j}, z_{j}\right)$, $j=1,2,3$. We will need to symmetrize the inequalities with respect to the action of $S_{3}$ diagonally permuting the variables $x_{1}, x_{2}, x_{3} ; y_{1}, y_{2}, y_{3} ; z_{1}, z_{2}, z_{3}$.

The subsystem associated to $H^{*}\left(G / P_{1}\right)$ (before symmetrization).

$$
\begin{aligned}
& x_{1} \leq x_{2}+x_{3}, \\
& y_{1} \leq y_{2}+x_{3}, \\
& z_{1} \leq z_{2}+x_{3}, \\
& z_{1} \leq y_{2}+y_{3} .
\end{aligned}
$$

After symmetrizing, we get 18 inequalities. 
SHRAWAN KUMAR

The subsystem associated to $H^{*}\left(G / P_{2}\right)$ (before symmetrization).

$$
\begin{aligned}
& x_{1}+y_{1} \leq x_{2}+y_{2}+x_{3}+y_{3} \\
& x_{1}+z_{1} \leq x_{2}+z_{2}+x_{3}+y_{3} \\
& y_{1}+z_{1} \leq y_{2}+z_{2}+x_{3}+y_{3} \\
& x_{1}+z_{2} \leq z_{1}+x_{2}+x_{3}+y_{3} \\
& y_{1}+z_{2} \leq z_{1}+y_{2}+x_{3}+y_{3} \\
& x_{1}+y_{2} \leq y_{1}+x_{2}+x_{3}+y_{3} \\
& y_{1}+z_{1} \leq x_{2}+z_{2}+x_{3}+z_{3} \\
& y_{1}+z_{2} \leq z_{1}+x_{2}+x_{3}+z_{3} \\
& x_{1}+z_{2} \leq y_{1}+x_{2}+x_{3}+z_{3} .
\end{aligned}
$$

We get 48 inequalities after symmetrizing.

The subsystem associated to $H^{*}\left(G / P_{3}\right)$ (before symmetrization).

$$
\begin{aligned}
& x_{1}+y_{1}+z_{1} \leq x_{2}+y_{2}+z_{2}+x_{3}+y_{3}+z_{3}, \\
& x_{1}+y_{1}+z_{2} \leq z_{1}+x_{2}+y_{2}+x_{3}+y_{3}+z_{3}, \\
& x_{1}+z_{1}+y_{2} \leq y_{1}+x_{2}+z_{2}+x_{3}+y_{3}+z_{3}, \\
& x_{1}+y_{2}+z_{2} \leq y_{1}+z_{1}+x_{2}+x_{3}+y_{3}+z_{3}, \\
& x_{1}+y_{2}+z_{3} \leq y_{1}+z_{1}+x_{2}+z_{2}+x_{3}+y_{3} .
\end{aligned}
$$

After symmetrizing we get 27 inequalities.

To summarize, for $B_{3}$, these provide an irredundant set of altogether 93 inequalities.

\subsection{The inequalities for $C_{3}$}

In this case the Weyl chamber $\mathfrak{h}_{+}$is given by the triples $(x, y, z)$ of real numbers satisfying $x \geq y \geq z \geq 0$. Here $x, y, z$ are the coordinates relative to the standard basis $\epsilon_{1}, \epsilon_{2}, \epsilon_{3}$ in the notation of [Bo, pp. 254-255]. The inequalities will now be in terms of $\left(v_{1}, v_{2}, v_{3}\right) \in \mathfrak{h}_{+}^{3}$ with $v_{j}=\left(x_{j}, y_{j}, z_{j}\right), j=1,2,3$. We will need to symmetrize the inequalities with respect to the action of $S_{3}$ diagonally permuting the variables $x_{1}, x_{2}, x_{3} ; y_{1}, y_{2}, y_{3} ; z_{1}, z_{2}, z_{3}$.

The subsystem associated to $H^{*}\left(G / P_{1}\right)$ (before symmetrization).

$$
\begin{aligned}
& x_{1} \leq x_{2}+x_{3}, \\
& y_{1} \leq y_{2}+x_{3}, \\
& z_{1} \leq z_{2}+x_{3}, \\
& z_{1} \leq y_{2}+y_{3} .
\end{aligned}
$$

These give 18 inequalities after symmetrization. 
The subsystem associated to $H^{*}\left(G / P_{2}\right)$ (before symmetrization).

$$
\begin{aligned}
& x_{1}+y_{1} \leq x_{2}+y_{2}+x_{3}+y_{3}, \\
& x_{1}+z_{1} \leq x_{2}+z_{2}+x_{3}+y_{3}, \\
& y_{1}+z_{1} \leq y_{2}+z_{2}+x_{3}+y_{3}, \\
& x_{1}+z_{2} \leq z_{1}+x_{2}+x_{3}+y_{3}, \\
& y_{1}+z_{2} \leq z_{1}+y_{2}+x_{3}+y_{3}, \\
& x_{1}+y_{2} \leq y_{1}+x_{2}+x_{3}+y_{3}, \\
& y_{1}+z_{1} \leq x_{2}+z_{2}+x_{3}+z_{3}, \\
& y_{1}+z_{2} \leq z_{1}+x_{2}+x_{3}+z_{3}, \\
& x_{1}+z_{2} \leq y_{1}+x_{2}+x_{3}+z_{3} .
\end{aligned}
$$

This subsystem after symmetrization consists of 48 inequalities.

The subsystem associated to $H^{*}\left(G / P_{3}\right)$ (before symmetrization).

$$
\begin{aligned}
& x_{1}+y_{1}+z_{1} \leq x_{2}+y_{2}+z_{2}+x_{3}+y_{3}+z_{3}, \\
& x_{1}+y_{1}+z_{2} \leq z_{1}+x_{2}+y_{2}+x_{3}+y_{3}+z_{3}, \\
& x_{1}+z_{1}+y_{2} \leq y_{1}+x_{2}+z_{2}+x_{3}+y_{3}+z_{3}, \\
& x_{1}+y_{2}+z_{2} \leq y_{1}+z_{1}+x_{2}+x_{3}+y_{3}+z_{3}, \\
& x_{1}+y_{2}+z_{3} \leq y_{1}+z_{1}+x_{2}+z_{2}+x_{3}+y_{3} .
\end{aligned}
$$

This gives 27 inequalities after symmetrization.

The 27 inequalities above can be rewritten in a very simple way. Let $S=$ $\sum_{j=1}^{3} x_{j}+y_{j}+z_{j}$. Then the 27 inequalities are just the inequalities

$$
x_{i}+y_{j}+z_{k} \leq \frac{S}{2}, i, j, k=1,2,3 .
$$

These 93 inequalities form an irredundant set to define $\bar{\Gamma}_{3}\left(C_{3}\right) \subset \mathfrak{h}_{+}^{3}$.

Remark 18. The irredundant set of inequalities to define $\bar{\Gamma}_{3}\left(\mathrm{D}_{4}\right) \subset \mathfrak{h}_{+}^{3}$ is explicitly determined in [KKM, §5]. 
SHRAWAN KUMAR

\title{
A. BUILDINGS AND \\ TENSOR PRODUCT MULTIPLICITIES
}

\author{
MICHAEL KAPOVICH \\ Department of Mathematics \\ University California \\ Davis, CA 95616, USA \\ kapovich@math.ucdavis.edu
}

The goal of this appendix is to explain connections between metric geometry (driven by notions such as distance and curvature) and the representation theory of complex semisimple Lie groups. The connections run through the theory of buildings. We will give sketches of proofs of results established in a sequence of papers $\left[\mathrm{KLM}_{1}\right],\left[\mathrm{KLM}_{2}\right],\left[\mathrm{KLM}_{3}\right],\left[\mathrm{KM}_{1}, \mathrm{KM}_{2}\right]$ of the author and his collaborators: B. Leeb and J. J. Millson. (The results were further extended in the papers [KKM], $[\mathrm{HKM}]$ and $[\mathrm{BeKa}]$.$) We also refer the reader to the survey [Kap] for a different take$ on these results and the discussion of symmetric spaces and eigenvalue problems which we did not discuss here. Some of this theory should generalize in the context of Kac-Moody groups; we refer the reader to [GR] for the first step in this direction. We refer the reader to the papers [FK], [FKK], [Kam], [MV] for other developments connecting algebraic geometry of buildings and representation theory.

Acknowledgements. Partial financial support for this work was provided by the NSF grants DMS-09-05802 and DMS-12-05312.

\section{A.1. Notation}

Throughout, we let $F$ be a local field with discrete valuation and $\mathcal{O} \subset \mathbb{K}$ be the corresponding ring of integers; the reader can think of $F=\mathbb{Q}_{p}, \mathcal{O}=\mathbb{Z}_{p}$. Let $q$ denote the cardinality of the residue field of $F$ and let $\pi \in F$ be a uniformizer. Let $\mathrm{G}$ be a split semisimple algebraic group-scheme over $\mathbb{Z}$, let $\mathrm{G}^{\vee}$ be the Langlands' dual group scheme and set

$$
G=\mathrm{G}(F), \quad G^{\vee}:=\mathrm{G}^{\vee}(\mathbb{C})
$$

We will also fix a dual maximal tori $T \subset G, T^{\vee} \subset G^{\vee}$ and Borel subgroups $B, B^{\vee}$ normalized by these tori. These choices will allow us to talk about (dominant) weights of the group $G^{\vee}$ (more precisely, weights of $T^{\vee}$ positive with respect to $\left.B^{\vee}\right)$, etc. Let $\mathrm{U} \subset \mathrm{G}$ be the unipotent radical, set $U:=\mathrm{U}(F)$.

We let $\mathfrak{X}_{*}(\mathrm{~T}), \mathfrak{X}^{*}(\mathrm{~T})$ denote the groups of cocharacters and characters of $\mathrm{T}$, a maximal torus of $\mathrm{G}$. The subgroup $K=\mathrm{G}(\mathcal{O})$ is a maximal compact subgroup of $G$. Lastly, let $W$ be the Weyl group of $\mathrm{G}$ corresponding to $\mathrm{T}$.

The general theme of this appendix is that the representation theory of the group $G^{\vee}$ is governed by the geometry of the group $G$ : This geometry will manifest itself through the geometry (both metric and algebraic) of the Bruhat-Tits buildings associated with the group $G$. 
Given the root system $R$ of the group $G$ (of rank $\ell$ ) we define the constant $k_{R}$ to the least common multiple of the numbers $a_{1}, \ldots, a_{\ell}$, where

$$
\theta=\sum_{i=1}^{\ell} a_{i} \alpha_{i}
$$

with simple roots $\alpha_{i}$ of $R$ and the highest root $\theta$ of $R$. For instance, if $R$ is an irreducible root system, then $R$ has type $\mathrm{A}$ if and only if $k_{R}=1$ and the largest value $k_{R}=60$ occurs for $R=\mathrm{E}_{8}$.

\section{A.2. Buildings}

In this section we discuss axioms of (discrete) Euclidean (affine) buildings.

Coxeter complexes. Let $A$ be the Euclidean $\ell$-dimensional space. By choosing the origin $o \in \mathbb{R}^{\ell}$ we will be identifying $A$ with the vector space $\mathbb{R}^{\ell}$ equipped with the standard inner product and associated Euclidean metric. Let $W_{\text {af }}$ be a Coxeter group acting properly discontinuously, isometrically and faithfully on $A$, so that Coxeter generators of $W_{\text {af }}$ act as reflections on $A$. Note that the stabilizer $W_{x}$ of a point $x \in A$ in the group $W_{\text {af }}$ is a finite reflection group. We will assume that the group $W_{\text {af }}$ acts cocompactly on $A$, i.e., that $W_{\text {af }}$ is crystallographic. The pair $\left(A, W_{\mathrm{af}}\right)$ is a Euclidean Coxeter complex; the space $A$ is called a model apartment. Fixed-point sets of reflections in $W_{\text {af }}$ are called walls in $A$. Let $\mathcal{W}$ denote the union of walls in $A$. Closures $a$ of components of the complement

$$
A \backslash \mathcal{W},
$$

are alcoves in $A$; they are fundamental domains for the action of $W_{\text {af }}$ on $A$.

A half-apartment in $A$ is a half-space bounded by a wall. The group $W_{\text {af }}$ splits as the semidirect product $\Lambda \rtimes W$, where $\Lambda$ is a group of translations in $\mathbb{R}^{\ell}$. Since $W_{\text {af }}$ was assumed to be crystallographic, it is associated to a root system $R$. Then, $\Lambda$ is a lattice in $A$, the coroot lattice $Q\left(R^{\vee}\right)$; the finite reflection group $W$ is the stabilizer of a point $o \in A$. The normalizer of $W_{\text {af }}$ in the full group of translations of $A$ is the coweight lattice $P\left(R^{\vee}\right)$.

We will fix a fundamental domain $\Delta$ (a positive Weyl chamber) for the action of $W$ on $A$, so that $\Delta$ is bounded by walls. We let $\Delta^{*} \subset \mathbb{R}^{\ell}$ denote the dual cone of $\Delta$ :

$$
\Delta^{*}=\left\{v \in \mathbb{R}^{\ell}:\langle v, u\rangle \geq 0, \forall u \in \Delta\right\} .
$$

The cone $\Delta^{*}$ is spanned by the positive root vectors of $R$.

We define a partial order

$$
u \leq_{\Delta^{*}} v
$$

on $\mathbb{R}^{\ell}$ by requiring that

$$
v-u \in \Delta^{*}
$$

The Coxeter complex has the natural structure of a regular cell complex, where facets are alcoves and vertices are zero-dimensional intersections of walls. By abusing the terminology, we will refer to this cell complex as a Coxeter complex as well. 


\section{SHRAWAN KUMAR}

A vertex of the model Euclidean apartment $A$ is called special if its stabilizer in $W_{\text {af }}$ is isomorphic to $W$, i.e., is the maximal possible. The root system $R$ is the product of root systems of type $A$, if and only if every vertex is special. The numbers $k_{R}$ are defined so that $k_{R}$ is the least natural number $n$ so that the image of every vertex $v \in A$ under the scaling $x \mapsto n x$, is a special vertex of $A$. For instance, for the root system of type $A$, every vertex is special, so $k_{R}=1$.

Affine buildings. A space modeled on a Coxeter complex $\left(A, W_{\mathrm{af}}\right)$ is a metric space $X$ together with a collection of isometric embeddings ("charts") $\phi: A \rightarrow X$, so that transition maps between charts are restrictions of elements of $W_{\text {af }}$. The number $\ell$ is the rank of $X$. Images of charts are called apartments in $X$. Note that apartments are (almost) never open in $X$. By taking images of vertices, walls and half-apartments in $A$ under charts, we define vertices, walls and half-apartments in $A$. An isometry $g: X \rightarrow X$ is an automorphism of the space $X$ if for every pair of charts $\phi, \psi$, the composition $\psi^{-1} \circ g \circ \phi$ is the restriction of an element of $W_{\mathrm{af}}$. Our definition of affine buildings follows [KL]; equivalence of this definition to the more combinatorial one (which could be found, e.g., in $[R]$ ) was established in $[\mathrm{Pa}]$. Note that the definition that we give below extends (by adding an extra axiom) to the case of non-discrete buildings; see $[\mathrm{KL}]$ and $[\mathrm{Pa}]$.

Definition 17. A (thick) Euclidean (affine) building is a space modeled on a Euclidean Coxeter complex and satisfying the three axioms listed below:

A1 (Connectivity). Every pair of points in $X$ belongs to a common apartment. A2 (Curvature bounds). We require $X$ to be a CAT(0)-metric space. (We will explain the definitions below.)

A3 (Thickness). Every wall in $X$ is the intersection of (at least) three halfapartments.

This definition parallels the one of the symmetric space $G / K$ of a (connected) semisimple Lie group. The CAT(0) condition is the analogue of the fact that symmetric spaces of noncompact type have sectional curvature $\leq 0$.

The CAT $(0)$ condition was first introduced by A. D. Alexandrov in the 1950s. Informally, this condition means that geodesic triangles in $X$ are thinner than the geodesic triangles in the Euclidean plane. Below is the precise definition.

A geodesic segment $x y$ in $X$ is an isometric (i.e., distance-preserving) embedding of an interval $[a, b] \subset \mathbb{R}$ into $X$; the points $x, y$ are the images of $a, b$ under this isometric embedding. We will orient the geodesic segment $x y$ from $x$ to $y$. Similarly, one defines geodesic rays as isometric maps $[0, \infty) \rightarrow X$.

An (oriented) geodesic triangle $\tau=x y z$ in $X$ is a concatenation of the three oriented geodesic segments $x y, y z, z x$, the edges of $\tau$; the points $x, y, z$ are the vertices of $\tau$. A disoriented geodesic triangle is formed by the segments $x y, y z, x z$. The side-lengths of a triangle $\tau$ are the lengths of its edges; they are denoted $|x y|,|y z|,|z x|$. Then the side-lengths of $\tau$ satisfy the triangle inequalities

$$
|x y| \leq|y z|+|z x| .
$$

The triangle inequalities above are necessary and sufficient for the existence of a geodesic triangle in $\mathbb{R}^{2}$ with the given side-lengths. Then, in $\mathbb{R}^{2}$ there exists a 


\section{EIGENVALUE PROBLEM}

comparison triangle for the triangle $\tau \subset X$, namely, a geodesic triangle $\widetilde{\tau}$ with vertices $\widetilde{x}, \widetilde{y}, \widetilde{z}$ whose side-lengths are the same as the side-lengths of $\tau$. Given any pair of points $p \in x y, q \in y z$, one defines the corresponding comparison points $\widetilde{p} \in \widetilde{x} \widetilde{y}$ and $\widetilde{q} \in \widetilde{y} \widetilde{z}$, so that

$$
|x p|=|\widetilde{x} \widetilde{p}|, \quad|y q|=|\widetilde{y} \widetilde{q}| .
$$

Then the space $X$ is said to be $\operatorname{CAT}(0)$ if for every geodesic triangle in $X$ we have:

$$
|p q| \leq \mid \widetilde{p} \widetilde{q} .
$$

We refer the reader to [Bll] for further details on CAT(0) geometry.

Example 2. Suppose that $X$ is a 1-dimensional Euclidean building. Then $X$ is a connected graph, whose vertices are the images of the walls in $A$ and all edges have the same length, which is the minimal distance between the walls in $A$. The thickness axiom is equivalent to the requirement that every vertex of $X$ has valence $\geq 3$. The curvature restriction is that the graph $X$ contains no circuits, i.e., $X$ is a tree.

Note that $X$ has the natural structure of a cell complex, where the cells are images of cells in $\left(A, W_{\mathrm{af}}\right)$. However, it is important to note that we consider all points of $X$, not just its vertices.

Chamber-valued distance function. Let $X$ be a Euclidean building. Our next goal is to define a 2-point invariant $d_{\Delta}(x, y)$ in $X$, taking values in the Weyl chamber $\Delta$. We first define $d_{\Delta}(x, y)$ for $x, y \in A$. We identify the affine space $A$ with the vector space $\mathbb{R}^{\ell}$ by declaring $o$ to be the origin. Next, identify the directed segment $\overrightarrow{x y}$ with a vector $v$ in $\mathbb{R}^{\ell}$, then project $v$ to a vector $\bar{v} \in \Delta$ using the action of the group $W \subset W_{\text {af }}$. We declare $\bar{v} \in \Delta$ to be the $\Delta$-valued distance $d_{\Delta}(x, y)$ between the points $x, y \in A$. It is clear that $d_{\Delta}$ is $W_{\mathrm{af}}$-invariant. Now, for a chart $\phi: A \rightarrow X$ we set

$$
d_{\Delta}(\phi(x), \phi(y)):=d_{\Delta}(x, y) .
$$

Since every two points in $X$ belong to a common apartment and transition maps between charts in $X$ are restrictions of elements of $W_{\text {af }}$, it follows that we obtain a well-defined function $d_{\Delta}: X \times X \rightarrow \Delta$. Furthermore, if $g$ is an automorphism of $X$, then $g$ preserves $d_{\Delta}$.

Note that, in general, the function $d_{\Delta}$ is not symmetric; however,

$$
d_{\Delta}(x, y)=-w_{0} d_{\Delta}(y, x)
$$

where $w_{0} \in W$ is the longest element of this finite Coxeter group. For an oriented geodesic segment $x y$ in $X$, we regard $d_{\Delta}(x, y)$ as the $\Delta$-valued lengths of $x y$. More generally, given a piecewise-geodesic path $p$ in $X$ (i.e., a concatenation of geodesic paths $\left.p_{i}, i=1, \ldots, m\right)$, we define the $\Delta$-length of $p$, denoted length ${ }_{\Delta}$, to be the sum

$$
\sum_{i=1}^{m} \operatorname{length}_{\Delta}\left(p_{i}\right) \in \Delta .
$$




\section{SHRAWAN KUMAR}

The "metric space" $\left(X, d_{\Delta}\right)$ has an interesting geometry. For instance, the generalized triangle inequalities for $X$ are necessary and sufficient conditions for the existence of an oriented triangle in $X$ with the given $\Delta$-side lengths $(\lambda, \mu, \nu) \in \Delta^{3}$. A priori, it is far from clear why such conditions are given by linear inequalities. It was proven in $\left[\mathrm{KLM}_{1}\right]$ that these necessary and sufficient conditions are exactly the inequalities defining the eigencone $C(H)$ for any (complex or real) semisimple Lie group $H$ whose Weyl group is isomorphic to $W$; below we will explain why this is true for the group $H=G^{\vee}$.

Not much is known about this "geometry" beyond the generalized triangle inequalities. For instance, one can ask to which extent this geometry is "nonpositively curved." Below is a partial result in this direction (reminiscent of the fact that the ordinary distance between geodesics in a $C A T(0)$ space is a convex function):

Theorem 66. The $\Delta$-distance function between geodesics in $X$ is $\Delta^{*}$-convex. More precisely: Let $\gamma_{1}(t), \gamma_{2}(t)$ be geodesics in $X$ parameterized with the constant speed. Define the function

$$
\varphi(t)=d_{\Delta}\left(\gamma_{1}(t), \gamma_{2}(t)\right) .
$$

Then for all $a, b$, and $t \in[0,1]$,

$$
\varphi((1-t) a+t b) \leq_{\Delta^{*}}(1-t) \varphi(a)+t \varphi(b) .
$$

We now continue with definitions. A vertex in the Euclidean building $X$ modeled on $\left(A, W_{\mathrm{af}}\right)$ is special if it is the image of a special vertex of the model apartment $A$ under a chart. A triangle $\tau$ in $X$ is called special if its vertices are special vertices of $X$ and $\Delta$-side lengths are elements of $\Delta \cap P\left(R^{\vee}\right)$.

We define

$$
\widehat{\mathcal{T}}_{\lambda, \mu, \nu}
$$

to be the space of oriented triangles in $X$ with the $\Delta$-valued side-lengths $\lambda, \mu, \nu$. Note that we do not require vertices of these triangles to be vertices of $X$. Similarly, we define

$$
\widehat{\mathcal{T}}_{\lambda, \mu, \nu}^{s p} \subset \widehat{\mathcal{T}}_{\lambda, \mu, \nu}
$$

to be the subset consisting of special triangles.

Spherical buildings at infinity. Spherical or Tits buildings are defined via axioms similar to the ones for Euclidean buildings, except the model space is no longer a Euclidean space equipped with an action of a reflection group, but a sphere equipped with an isometric action of a finite reflection group. A spherical building $Y$ is a cell complex whose faces are isometric to faces of the spherical Weyl chamber $\Delta_{s p h} \subset S^{\ell-1}$, a fundamental domain for $W$. (The Weyl chamber $\Delta$ of $W$ is the Euclidean cone over $\Delta_{s p h}$.) Then $Y$ admits a simplicial projection $\theta: Y \rightarrow \Delta_{s p h}$. The image $\theta(\xi)$ is called the type of a point $\xi \in Y$. What will be most important for us is that every Euclidean building $X$ has the ideal boundary $Y=\partial_{\text {Tits }} X$ which has the natural structure of a spherical building modeled on the sphere $S^{\ell-1}$ equipped with the action of the finite Weyl group $W$. Every geodesic ray $\rho:[0, \infty) \rightarrow X$ in 


\section{EIGENVALUE PROBLEM}

$X$ determines a point at infinity $\rho(\infty) \in Y$. Two rays $\rho_{1}, \rho_{2}$ determine the same point if they are asymptotic, i.e., the distance function

$$
d(t)=\left|\rho_{1}(t) \rho_{2}(t)\right|
$$

is bounded. One of the key geometric properties of $X$ that we need is that for two asymptotic rays, the distance function $d(t)$ is nonincreasing. This monotonicity property is implied by the $\operatorname{CAT}(0)$ property of $X$.

The angular (Tits) metric on $Y$ is denoted $\angle$. For instance, if $X$ is a tree, then $Y$ has the discrete metric which takes only the values 0 and $\pi$. If $X$ is the rank 2 affine building associated with the group $\mathrm{SL}(3, F)$ ( $W$ is the permutation group $S_{3}$ in this case), then $Y$ is a metric bipartite graph where every edge has length $\pi / 3$. Vertices of $Y$ are points and lines in the projective plane $P^{2}(F)$. Two vertices are connected by an edge iff they are incident.

A similar construction works for nonpositively curved symmetric spaces $X$ : Every such space admits the ideal boundary $\partial_{\text {Tits }} X$ which has the natural structure of a spherical building.

\section{A.3. Weighted configurations and stability}

Suppose that $\xi_{1}, \ldots, \xi_{n}$ are points in a spherical building $Y$ equipped with masses $m_{1}, \ldots, m_{n}$, which are nonnegative numbers. Given such weighted configuration $\psi$ in $Y$ we define $\left(\right.$ see $\left.\left[\mathrm{KLM}_{1}\right]\right)$ the slope function

$$
\operatorname{slope}_{\psi}(\eta)=-\sum_{i=1}^{n} m_{i} \cos \left(\angle\left(\xi_{i}, \eta\right)\right) .
$$

Definition 18. A weighted configuration $\psi$ is called (metrically) semistable if slope $_{\psi}(\eta) \geq 0$ for every $\eta$.

This condition is introduced in $\left[\mathrm{KLM}_{1}\right]$ in order to characterize properness of certain functions on $X$, namely, weighted Busemann functions associated to $\psi$. These functions can be defined for more general finite measures on ideal boundaries of $\operatorname{CAT}(0)$ spaces, and they play important roles in complex analysis (they were first introduced by Douady and Earle in the context of Teichmüller theory) and Riemannian geometry.

Note that a positive multiple of a semistable configuration is again semistable. For instance, if $X$ is a tree then $\psi$ is semistable if and only if the total mass of any point in $Y$ does not exceed half of the total mass

$$
\sum_{i=1}^{n} m_{i}
$$

of $\psi$. (If some points $\xi_{i}$ coincide, their masses, of course, add.)

It turns out (see $\left[\mathrm{KLM}_{1}\right]$ ) that the metric notion of semistability is essentially equivalent to Mumford's definition, once we introduce an algebraic group acting on $Y$. 


\section{SHRAWAN KUMAR}

Gauss correspondence. Let $\Pi=x_{1} x_{2} x_{3} \ldots x_{n}$ denote an oriented geodesic polygon in $X$ with vertices $x_{i}$ and edges $x_{i} x_{i+1}, i$ is taken modulo $n$. (The reader can assume that $n=3$ since we are primarily interested in triangles in $X$.) We then extend every edge $x_{i} x_{i+1}$ of $\Pi$ to a geodesic ray $\rho_{i}$ starting at $x_{i}$ and representing a point $\xi_{i}=\rho_{i}(\infty) \in Y$. We assign the weight $m_{i}=\left|x_{i} x_{i+1}\right|$ to $\xi_{i}$. The ray $\rho_{i}$ is nonunique, but this will not concern us; what is important is that the type $\theta\left(\xi_{i}\right)$ is well defined (unless $m_{i}=0$ ). We note that $\theta\left(\xi_{i}\right)$ is the unit vector which has the same direction as

$$
\lambda_{i}=d_{\Delta}\left(x_{i}, x_{i+1}\right) \in \Delta .
$$

Thus,

$$
\lambda_{i}=m_{i} \theta\left(\xi_{i}\right) .
$$

The multivalued map

$$
\text { Gauss : } \Pi \mapsto \psi
$$

is the Gauss correspondence. (The picture defining this correspondence first appears in the letter from Gauss to Bolyai; see [G].) The following is the key result relating polygons in $X$ and weighted configurations in $Y$.

Theorem $67\left(\left[\mathrm{KLM}_{1}\right]\right)$. Every weighted configuration $\psi \in \operatorname{Gauss}(\Pi)$ is semistable. Conversely, for every semistable weighted configuration $\psi$ in $Y$, there exists a polygon in $X$ (with the metric side-lengths $\left.m_{i}\right)$ so that $\psi \in \operatorname{Gauss}(\Pi)$.

Below we describe what is involved in proving the hard direction in this theorem, namely, the converse implication. (It is very instructive to see why the theorem holds in the rank 1 case.)

Our goal is to "invert Gauss maps", i.e., given a semistable weighted configuration $\psi$, we would like to find a closed geodesic $n$-gon $\Pi$ so that $\psi \in \operatorname{Gauss}(\Pi)$. The polygons $\Pi$ correspond to the fixed points of a certain dynamical system on $X$ that we describe below. For $\xi \in Y=\partial_{\text {Tits }} X$ and $t \geq 0$, define the map $\phi:=\phi_{\xi, t}: X \rightarrow X$ by sending $x$ to the point at distance $t$ from $x$ on the geodesic ray $x \xi$ starting from $x$ and asymptotic to $\xi$. Since $X$ is $C A T(0)$, the map $\phi$ is 1-Lipschitz (recall that the distance function between asymptotic rays is decreasing). Then, given a weighted configuration $\psi$ with nonzero total mass, define the map

$$
\Phi=\Phi_{\psi}: X \rightarrow X
$$

as the composition

$$
\phi_{\xi_{n}, m_{n}} \circ \cdots \circ \phi_{\xi_{1}, m_{1}}
$$

The fixed points of $\Phi$ are the first vertices of closed polygons $\Pi=x_{1} \ldots x_{n}$ so that $\psi \in \operatorname{Gauss}(\Pi)$. Since the map $\Phi$ is 1-Lipschitz, and the space $X$ is complete $C A T(0)$, the map $\Phi$ has a fixed point if and only if the dynamical system $\left(\Phi^{i}\right)_{i \in \mathbb{N}}$ has a bounded orbit; see $\left[\mathrm{KLM}_{2}\right]$. Of course, in general, there is no reason to expect that $\left(\Phi^{i}\right)_{i \in \mathbb{N}}$ has a bounded orbit: For instance, if the configuration $\psi$ is supported at a single point, all orbits are unbounded. The following theorem was proven for locally compact buildings in the original version of $\left[\mathrm{KLM}_{2}\right]$ and by Andreas Balser [Bls] in the general case: 


\section{EIGENVALUE PROBLEM}

Theorem 68. Suppose that $X$ is a Euclidean building. Then $\psi$ is semistable if and only if $\left(\Phi^{i}\right)_{i \in \mathbb{N}}$ has a bounded orbit.

An algebraically inclined reader can ignore all the material in this section except for the following corollary, whose only known proof goes through the stability theory and Gauss correspondence described above:

Corollary 69. Let $X$ be a Euclidean building. Let $\lambda, \mu, \nu$ be dominant weights such that

$$
\lambda+\mu+\nu \in Q\left(R^{\vee}\right)
$$

Take $N \in \mathbb{N}$ and set

$$
\left(\lambda^{\prime}, \mu^{\prime}, \nu^{\prime}\right)=N(\lambda, \mu, \nu)
$$

Suppose that the space $\widehat{T}_{\lambda^{\prime}, \mu^{\prime}, \nu^{\prime}} \neq \varnothing$. Then there exists a triangle $\tau \in \widehat{T}_{\lambda, \mu, \nu}$ so that vertices of $\tau$ are vertices of $X$.

\section{A.4. Buildings and algebraic groups}

The (so far, purely geometric) theory of buildings connects to the theory of algebraic groups as follows. Given a group $G=\mathrm{G}(F)$ as above, Bruhat and Tits [BT] associate with $G$ a Euclidean (Bruhat-Tits) building $X$, so that $G$ acts by automorphisms on $X$. The action $G \curvearrowright X$ is transitive on the set of apartments in $X$. For each apartment $A \subset X$ we define $G_{A}$ to be the stabilizer of $A$ in $G$. Then, the image of $G_{A}$ in $A u t(A)$ (under the restriction map) is equivariantly isomorphic to the group $L \rtimes W$, so that

$$
Q\left(R^{\vee}\right) \subset L \rtimes W \subset W_{\text {af }} \subset P\left(R^{\vee}\right) \rtimes W
$$

and $L$ is the cocharacter lattice of $\mathrm{G}$. The maximal split torus $T$ in $G$ preserves one of the apartments $A \subset X$ and acts on $A$ as the lattice $L$. Furthermore, the group $K$ stabilizes a certain special vertex $o \in X$; the $G$-orbit $\operatorname{Gr}_{G}:=G \cdot o=G / K$ is called the affine Grassmannian of $G$.

The spherical building $Y=\partial_{\text {Tits }} X$ is recovered from $G$ as follows: Stabilizers of faces of $X$ are parabolic subgroups of $G$; stabilizers of facets are conjugates of the Borel subgroup $B$. Thus, the space of facets in $Y$ is naturally identified with the quotient $G / B$, the set of $F$-points in the complete flag variety $\mathrm{G} / \mathrm{B}$.

The group $G$ has the Cartan decomposition

$$
G=K T_{+} K
$$

where $T_{+}$is a subsemigroup in the torus $T$ consisting of elements of the form

$$
\chi(\pi), \quad \chi \in L_{+},
$$

where $\pi \in F$ is a uniformizer (note that $\chi$ 's are cocharacters of T). Thus, we obtain the Cartan projection

$$
c: G \rightarrow L_{+}, \quad c(g)=\chi(\pi)
$$




\section{SHRAWAN KUMAR}

In the case $G=\mathrm{SL}(\ell+1)$, the Cartan decomposition is just another interpretation of the Smith normal form for elements of $G$; thus, we will think of the vector $c(g)$ as the set of invariant factors of $g$.

We can now give a GIT interpretation of semistability for weighted configurations in $Y$. For simplicity, we assume that each point $\xi_{i} \in Y$ is a regular point, i.e., it belongs to a unique facet $\sigma_{i}$ of $Y$; thus, $\sigma_{i} \in G / B$. We will also assume that each vector

$$
\lambda_{i}=m_{i} \theta\left(\xi_{i}\right) \in \Delta
$$

belongs to the lattice $L$. In particular, $\lambda_{i}$ determines a line bundle $\mathcal{L} \lambda_{i}$ on $G / B$. Then

A weighted configuration $\psi$ is metrically semistable if and only if the $n$-tuple of chambers

$$
\left(\sigma_{1}, \ldots, \sigma_{n}\right) \in(G / B)^{n}
$$

is semistable in the GIT sense with respect to the diagonal action of the $G$, where we use the tensor product of the line bundles $\mathcal{L} \lambda_{i}$ to define the polarization.

A similar statement holds for general weighted configurations in $Y$, except we have to use the product of (possibly) partial flag varieties.

We now can give the first algebraic interpretation of oriented polygons $\Pi=$ $x_{1} \ldots x_{n}$ (with $x_{1}=o$ ) in $X$ whose vertices belong to $\mathrm{Gr}_{G}$ : Every polygon $\Pi$ with $\Delta$-side lengths $\lambda_{1}, \ldots, \lambda_{n}$ determines a tuple of elements $g_{1}, \ldots, g_{n} \in G$ such that

$$
c\left(g_{i}\right)=\lambda_{i}, i=1, \ldots n
$$

and

$$
g_{1} \cdots g_{n}=1
$$

Conversely, every tuple $\left(g_{1}, \ldots, g_{n}\right)$ determines a polygon as above. It turns out that instead of constructing polygons with vertices in $\mathrm{Gr}_{G}$, it suffices to construct special polygons $X$. We refer to $\left[\mathrm{KLM}_{2}\right]$ and $\left[\mathrm{KLM}_{3}\right]$ for the details.

We then define two sets

$$
\operatorname{Hecke}(G) \subset \operatorname{Tri}(X),
$$

where $\operatorname{Hecke}(G)$ consists of triples $\left(\lambda_{1}, \lambda_{2}, \lambda_{3}\right) \in L_{+}^{3}$ such that there exists a special oriented triangle in $X$ with $\Delta$-side lengths $\lambda_{1}, \lambda_{2}, \lambda_{3}$, while $\operatorname{Tri}(X)$ consists of triples $\left(\lambda_{1}, \lambda_{2}, \lambda_{3}\right) \in \Delta^{3}$ such that there exists an oriented triangle in $X$ with $\Delta$ side lengths $\lambda_{1}, \lambda_{2}, \lambda_{3}$. (In the next section we will see why the latter set has the name Hecke.) For now, we just record the (easy) fact that

$$
\operatorname{Hecke}(G) \subset\left\{\left(\lambda_{1}, \lambda_{2}, \lambda_{3}\right): \lambda_{1}+\lambda_{2}+\lambda_{3} \in Q\left(R^{\vee}\right)\right\}
$$

see $\left[\mathrm{KLM}_{3}\right]$ for two different proofs (geometric and algebraic). Observe also that, by considering disoriented special triangles in $X$, we can interpret the set $\operatorname{Hecke}(G)$ as answering the following algebraic problem:

- Given two sets of invariant factors $\lambda_{1}, \lambda_{2} \in L_{+}$, describe possible invariant factors of the products $g_{1} g_{2}$, where $c\left(g_{1}\right)=\lambda_{1}, c\left(g_{2}\right)=\lambda_{2}$. 
In this survey we discuss two ways in which special triangles in Euclidean buildings $X$ connect to the representation theory of the group $G^{\vee}$ :

- Satake correspondence.

- Littelmann path model.

\section{A.5. Hecke rings, Satake transform and triangles in buildings}

In this section we describe the Satake transform from the (spherical) Hecke ring of $G$ to the representation ring of $G^{\vee}$. We refer the reader to [Gro] for more details. (There are more general notions of Satake transform which apply to other discrete valued fields, like $\mathbb{C}((t))$; these generalizations require one to work with sheaves.)

Satake transform. Below we describe an integral transform $S$, the Satake transform, from a ring $\mathcal{H}_{G}$ (spherical Hecke ring) of compactly supported, $K$-biinvariant functions on $G$ to the ring of left $K$-invariant, right $U$-invariant functions on $G$. The space of functions $\mathcal{H}_{G}$ is equipped with the convolution product

$$
f \star g(z)=\int_{G} f(x) \cdot g\left(x^{-1} z\right) d x
$$

where $d x$ is the Haar measure on $G$ giving $K$ volume 1 . Then $\left(\mathcal{H}_{G}, \star\right)$ is a commutative and associative ring.

Let $\delta: B \rightarrow \mathbb{R}_{+}^{*}$ be the modular function of $B$. We may regard $\delta$ as a left $K$-invariant, right $U$-invariant function on $G$. By the Iwasawa decomposition for $G$, any such function is determined by its restriction to $T$. We normalize the Haar measure $d u$ on $U$ so that the open subgroup $K \cap U$ has measure 1 . For a compactly supported $K$-biinvariant function $f$ on $G$ we define its Satake transform as a function $S f(g)$ on $G$ given by

$$
S f(g)=\delta(g)^{1 / 2} \cdot \int_{U} f(g u) d u .
$$

(The reader can think of $S$ as a generalization of the Fourier transform.) Then $S f$ is a left $K$-invariant, right $U$-invariant function on $G$ with values in $\mathbb{Z}\left[q^{1 / 2}, q^{-1 / 2}\right]$; this function is determined by its restriction to $T / T \cap K \cong \mathfrak{X}_{*}(\underline{T})$. Let $R\left(G^{\vee}\right) \cong$ $\left(\mathbb{Z}\left[\mathfrak{X}_{*}(\mathrm{~T})\right]\right)^{W}$ be the representation ring of $G^{\vee}$. Then:

Theorem 70. The image of $S$ lies in the subring

$$
\left(\mathbb{Z}\left[\mathfrak{X}_{*}(\mathrm{~T})\right]\right)^{W} \otimes \mathbb{Z}\left[q^{1 / 2}, q^{-1 / 2}\right]
$$

and $S$ defines a ring isomorphism

$$
S: \mathcal{H}_{G} \otimes \mathbb{Z}\left[q^{1 / 2}, q^{-1 / 2}\right] \rightarrow\left(\mathbb{Z}\left[\mathfrak{X}_{*}(\mathrm{~T})\right]\right)^{W} \otimes \mathbb{Z}\left[q^{1 / 2}, q^{-1 / 2}\right] \cong R\left(G^{\vee}\right) \otimes \mathbb{Z}\left[q^{1 / 2}, q^{-1 / 2}\right]
$$

Connection to geometry. How does a Hecke ring relate to the geometry of buildings? Functions on $G$ which are right-invariant under $K$ are the same thing as functions on the affine Grassmannian $\mathrm{Gr}_{G}$, while $K$-biinvariant functions are the 


\section{SHRAWAN KUMAR}

same thing as functions on the cone of dominant cocharacters $L^{+} \subset L=\mathfrak{X}_{*}(\mathrm{~T})$, i.e., functions on the set

$$
\Delta \cap L \cdot o \subset A
$$

where $A$ is a model apartment of $X$ and $\Delta$ is the positive chamber corresponding to our choice of the Borel subgroup B. We let $x_{\mu} \in A$ denote the vertex corresponding to the image of $o$ under the translation of $A$ given by the cocharacter $\mu$. Thus, we can identify $K$-orbits in $\operatorname{Gr}_{G}$ as "spheres with fixed $\Delta$-radius" $\mathbb{S}_{\mu}(o)$ :

$$
K \cdot x_{\mu}=\mathbb{S}_{\mu}(o)=\left\{x \in X^{0}: d_{\Delta}(o, x)=\mu\right\},
$$

where $X^{0}$ is the vertex set of $X$. More generally, for a vertex $u \in \operatorname{Gr}_{G}$ we set

$$
\mathbb{S}_{\mu}(u):=\left\{x \in X^{0}: d_{\Delta}(u, x)=\mu\right\} .
$$

The affine Grassmannian $\mathrm{Gr}_{G}$ has the structure of $F_{q}$-points of an ind-scheme, where the "spheres" $\mathbb{S}_{\mu}(u)$ are algebraic subvarieties (see $\left[\mathrm{H}_{1}\right]$ ). The closures of these subvarieties also have geometrically appealing interpretation as "closed metric balls"

$$
\overline{\mathbb{S}_{\mu}(u)}=\mathbb{B}_{\mu}(u)=\left\{x \in \operatorname{Gr}_{G}: d_{\Delta}(u, x) \leq_{\Delta^{*}} \mu\right\} .
$$

The cocharacters $\mu \in L$ define functions $c_{\mu} \in \mathcal{H}_{G}$, where $c_{\mu} \mid \Delta$ is the characteristic function of the singleton $\left\{x_{\mu}\right\}$. Since $\mathcal{H}_{G}$ consists of compactly supported functions, the functions $c_{\mu}$ form a basis in $\mathcal{H}_{G}$. In particular, we get the Hecke structure constants

$$
\begin{aligned}
m_{\lambda, \mu}^{\eta} & \in \mathbb{Z}_{+}, \\
c_{\lambda} \star c_{\mu} & =\sum_{\eta} m_{\lambda, \mu}^{\eta} c_{\eta} .
\end{aligned}
$$

Of course, these constants completely determine the ring $\mathcal{H}_{G}$. We next interpret the constants $m_{\lambda, \mu}^{\eta}$ in terms of the geometry of the building $X$.

Spaces of special triangles. Fix vectors $\lambda, \mu, \eta \in L_{+}$. Then every disoriented special triangle $a b c$ with vertices in $\operatorname{Gr}_{G} \subset X$ and $\Delta$-side-lengths $d_{\Delta}(a, b)=\lambda, d_{\Delta}(b, c)=$ $\mu, d_{\Delta}(a c)=\eta$ can be transformed (via an element of $G$ ) to a disoriented triangle of the form

$$
\text { oyx } x_{\eta}
$$

where the first and the last vertices are fixed and the vertex $y$ is variable. We let

$$
\mathcal{T}_{\lambda, \mu}^{\eta}\left(F_{q}\right)
$$

denote the space of such triangles. Similarly, for $\nu=\eta^{*}$ we define the space of oriented triangles

$$
\mathcal{T}_{\lambda, \mu, \nu}\left(F_{q}\right)
$$

of the form oyx $x_{\eta}$ with the $\Delta$-side-lengths $\lambda, \mu, \nu$. Let $f(q)=m_{\lambda, \mu, \nu}(q)$ denote the cardinality of the latter set.

Then $\left(\right.$ see $\left.\left[\mathrm{KLM}_{3}\right]\right)$ : 


\section{EIGENVALUE PROBLEM}

Lemma 71. $m_{\lambda, \mu}^{\eta}$ is the cardinality of the set $\mathcal{T}_{\lambda, \mu}^{\eta}\left(F_{q}\right)$.

Thus,

$$
\operatorname{Hecke}(G):=\left\{(\lambda, \mu, \nu): m_{\lambda, \mu}^{\nu^{*}} \neq 0\right\},
$$

which explains the name: This set answers the problem of describing the weights $\eta=\nu^{*}$ which appear with nonzero coefficient in the expansion of the product $c_{\lambda} \star c_{\mu}$ in terms of the basis $\left\{c_{\eta}\right\}$ of the Hecke ring $\mathcal{H}$.

Even though $S$ is an isomorphism, the relation between the structure constants of the Hecke ring of $G$ and the character ring of $G^{\vee}$ is somewhat indirect. Define

$$
n_{\lambda, \mu, \nu}=\operatorname{dim}\left(V_{\lambda} \otimes V_{\mu} \otimes V_{\nu}\right)^{G^{\vee}} .
$$

Let $\rho$ denote the half-sum of positive roots in $R^{\vee}$.

Theorem $72\left(\left[\mathrm{KLM}_{3}\right]\right) \cdot f(q)$ is a polynomial function of $q$ of degree $\leq q^{\langle\rho, \lambda+\mu+\nu\rangle}$ so that

$$
f(q)=n_{\lambda, \mu, \nu} q^{\langle\rho, \lambda+\mu+\nu\rangle}+\text { lower order terms. }
$$

In particular, if $n_{\lambda, \mu, \nu} \neq 0$ then $m_{\lambda, \mu, \nu}(q) \neq 0$ and, hence, $\mathcal{T}_{\lambda, \mu, \nu} \neq \varnothing$. In other words, if

$$
V_{\eta} \subset\left(V_{\lambda} \otimes V_{\mu}\right)^{G^{\vee}}
$$

then there exists a triangle in $X$ with special vertices and $\Delta$-side lengths $\lambda, \mu, \nu=\eta^{*}$.

Furthermore, if we replace the finite field $F_{q}$ with the algebraically closed field $\bar{F}_{q}$, then the space of triangles $\mathcal{T}_{\lambda, \mu, \nu}\left(\bar{F}_{q}\right)$ in the corresponding affine Grassmannian becomes an algebraic variety of dimension $\leq q^{\langle\rho, \lambda+\mu+\nu\rangle}$ and the number $n_{\lambda, \mu, \nu}$ is the number of components of $\mathcal{T}_{\lambda, \mu, \nu}\left(\bar{F}_{q}\right)$ which have the dimension $q^{\langle\rho, \lambda+\mu+\nu\rangle}$ (see $\left.\left[\mathrm{H}_{1}\right]\right)$.

The reverse relation between triangles in $X$ and tensor product decomposition is more subtle: For all simple complex Lie groups $G^{\vee}$ of non-simply laced type, there are examples where $\mathcal{T}_{\lambda, \mu, \nu}$ is nonempty while $n_{\lambda, \mu, \nu}=0$ (see Theorem 84).

We define the semigroup

$$
\operatorname{Rep}\left(G^{\vee}\right):=\left\{(\lambda, \mu, \nu): n_{\lambda, \mu, \nu} \neq 0\right\} .
$$

(Note that $\operatorname{Hecke}(G)$ need not be a semigroup; see $\left[\mathrm{KM}_{1}\right]$.) Thus, we have the inclusions

$$
\operatorname{Rep}\left(G^{\vee}\right) \subset \operatorname{Hecke}(G) \subset\left(L_{+}\right)^{3} \cap\left\{\lambda+\mu+\nu \in Q\left(R^{\vee}\right)\right\} .
$$

\section{A.6. Littelmann path model}

Let $A, W_{\text {af }}, R, G^{\vee}$, etc., be as in section A.2. Littelmann, in the series of papers $\left[\mathrm{L}_{1}\right],\left[\mathrm{L}_{2}\right],\left[\mathrm{L}_{3}\right]$, defined a path model for the representation ring of the group $G^{\vee}$. The key to this model is the notion of $L S$ paths in $A$. Below, we will give a definition of LS paths following $\left[\mathrm{KM}_{2}\right]$. This definition is essentially equivalent to Littelmann's definition (one difference is that we do not insist on the end-points of the path being in the coroot lattice); however, we do not explain the action of root operators on LS paths. 


\section{SHRAWAN KUMAR}

Hecke and LS paths. Every LS path is a piecewise-linear path $p:[0,1] \rightarrow A$ in $A$ satisfying several conditions. For every $t \in[0,1]$ we define two derivatives $p_{-}^{\prime}(t), p_{+}^{\prime}(t)$ : These are the derivatives on the left and on the right respectively. We will assume that $p(t)$ has constant speed, i.e., the Euclidean norm $\left|p^{\prime}(t)\right|$ is constant; in particular,

$$
\left|p_{-}^{\prime}(t)\right|=\left|p_{+}^{\prime}(t)\right|, \quad \forall t .
$$

For every $t$ we also have the finite Coxeter group $W_{p(t)}$, the stabilizer of $p(t)$ in $W_{\text {af }}$.

Axiom 1 ("Billiardness"). The path $p$ is a billiard path: For every $t \in[0,1]$, the vectors $p_{-}^{\prime}(t), p_{+}^{\prime}(t)$ belong to the same $W_{p(t)}$-orbit, i.e., there exists $w \in W_{p(t)}$ so that $w\left(p_{-}^{\prime}(t)\right)=p_{+}^{\prime}(t)$.

For instance, if $w$ is a single reflection in a wall $H$ passing through $p(t)$, then the above condition simply says that the path $p$ bends at the point $t$ according to the rules of optics (i.e., by the reflection in the wall $H$, the "mirror" or the "side of the billiard table").

Since $W_{p(t)}$ is generated by reflections, Axiom 1 implies that $w \in W_{p(t)}$ can be factored as a product of affine reflections

$$
w=\tau_{k} \circ \ldots \circ \tau_{1}
$$

in the group $W_{p(t)}$, where the derivative of each $\tau_{i}$ is a reflection $\tau_{\beta_{i}} \in W$ corresponding to a positive root $\beta_{i} \in R$. In particular, we obtain a chain of vectors

$$
u_{0}:=p_{-}^{\prime}(t), u_{1}:=\tau_{\beta_{1}}\left(u_{0}\right), \ldots, u_{k}=p_{+}^{\prime}(t)=\tau_{\beta_{k}}\left(u_{k-1}\right) .
$$

Definition 19. Let $W^{\prime} \subset W$ be a reflection subgroup. A finite sequence of vectors $u_{0}, \ldots, u_{k}$ in $\mathbb{R}^{\ell}$ is called a positive $W^{\prime}$-chain from $u_{0}$ to $u_{k}$ if for each $i \geq 1$ there exists a reflection $\tau_{\beta_{i}} \in W^{\prime}$ (corresponding to a positive root $\beta_{i}$ ) such that $\tau_{\beta_{i}}\left(u_{i-1}\right)=u_{i}$ and

$$
u_{i} \geq_{\Delta *} u_{i-1},
$$

i.e., $u_{i}-u_{i-1}$ is a positive multiple of $\beta_{i}$. In particular,

$$
u_{k} \geq_{\Delta *} u_{0} .
$$

A positive $W^{\prime}$-chain is called $W$-maximal if it cannot be refined to a larger positive $W$-chain from $u_{0}$ to $u_{k}$.

Clearly, every positive $W$-chain can be refined to a positive $W$-chain which is maximal. However, this is not the case for arbitrary positive $W^{\prime}$-chains where $W^{\prime} \neq W$. For the group $W_{p(t)}$ we let $W_{p(t)}^{\prime}$ denote the subgroup of $W$ consisting of derivatives (i.e., linear parts) of elements of $W_{p(t)}$.

Axiom 2 ("Positivity"). A billiard path $p$ is called a positively folded (or Hecke) path, if for every $t$ there exists a positive $W_{p(t)}^{\prime}$-chain from $p_{-}^{\prime}(t)$ to $p_{+}^{\prime}(t)$.

Geometrically speaking, positivity of the path $p$ means that at each break-point, the derivative $p_{+}^{\prime}(t) \in T_{p(t)}(A) \cong \mathbb{R}^{\ell}$ is obtained from $p_{-}^{\prime}(t) \in T_{p(t)}(A)$ by applying 
a sequence of reflections fixing $p(t)$, so that each reflection moves the corresponding vectors $u_{i-1}$ further towards the positive chamber $\Delta$.

Axiom 3 ("Maximality"). A positive $W_{p(t)}^{\prime}$-chain in Axiom 2 can be found, which is $W$-maximal.

From the geometric viewpoint, this is a strange axiom: It is defined in terms of inability to further refine positive $W_{p(t)}^{\prime}$-chains even if we are allowed to use reflections in $W$ which need not be reflections fixing $p(t)$ and, hence, have nothing to do with the fold made by the path $p$ at the point $p(t)$.

Note that Axiom 3 is satisfied automatically at each point $p(t)$ which is a special vertex of the apartment $A$.

Definition 20. A piecewise-linear path in $A$ is called an $L S$-path if it satisfies Axioms 1, 2 and 3. A path satisfying Axioms 1 and 2 is called a Hecke path.

Littelmann's path model for tensor product multiplicities. Given points $x, y \in A$ and a vector $\mu \in \Delta$, one considers the collection $L S_{x, y, \mu}$ of LS paths $p$ in $A$ connecting $x$ to $y$, so that length $\Delta(p)=\mu$. Similarly, one defines the set of Hecke paths Hecke ${ }_{x, y, \mu}$ of Hecke paths connecting $x$ to $y$.

If $x, y$ belong to $\Delta$, we consider the subset $L S_{x, y, \mu}^{+} \subset L S_{x, y, \mu}$ consisting of positive paths, i.e., paths whose image is contained in $\Delta$. For a weight $\eta \in L$ we let $x_{\eta} \in A$ denote the point so that $\overrightarrow{o x_{\eta}}=\beta$. Given a weight $\gamma \in L_{+}$we let $V_{\gamma}$ denote the (finite-dimensional) irreducible representation of $G^{\vee}$ with the highest weight $\gamma$.

Theorem 73 (P. Littelmann, $\left.\left[\mathrm{L}_{2}\right]\right)$. Let $\lambda, \mu \in L_{+} \subset P\left(R^{\vee}\right)$ be weights for the group $G^{\vee}$. Then

$$
V_{\lambda} \otimes V_{\mu}=\bigoplus_{\eta \in L_{+}} n_{\lambda, \mu}^{\eta} V_{\eta}
$$

where the multiplicity $n_{\lambda, \mu}^{\eta}$ equals the cardinality of $L S_{x_{\lambda}, x_{\eta}, \mu}^{+}$.

We will call the "broken triangle" in $\Delta$ which is the concatenation of the geodesic segment $o x_{\lambda}$, a piecewise-geodesic path $p \in L S_{x_{\lambda}, x_{\nu}, \mu}^{+}$and the geodesic segment $x_{\eta} o$, a Littelmann triangle. Similarly, we define Hecke triangles by replacing LS paths with Hecke paths.

Remark 19. The spaces of Littelmann and Hecke triangles are invariant under scaling by natural numbers.

1-skeleton paths. In $\left[\mathrm{KM}_{2}\right]$, in order to get a better connection between the Littelmann path model and triangles in buildings, we had to modify slightly the concept of LS paths. (This modification is actually a special case of a more general class of paths defined by Littelmann earlier in terms of root operators.) Namely, we will have to relax Axiom 1 in the definition (and accordingly modify Axiom 2). Let $\varpi_{1}, \ldots, \varpi_{\ell}$ denote the fundamental weights of $R$. Then every positive weight $\mu$ of $G$ is the sum

$$
\mu=\sum_{i=1}^{\ell} \mu_{i}, \quad \mu_{i}=c_{i} \varpi_{i}, \quad c_{i} \in \mathbb{Z}_{+}, \quad i=1, \ldots, \ell
$$




\section{SHRAWAN KUMAR}

We then obtain a model path $p_{\mu}$ in $\Delta$ as the concatenation of the geodesic segments $p_{i}$ connecting $o$ to $x_{\mu}$, where each $p_{i}$ is a translate of the geodesic segment $o x_{\mu_{i}}$. Thus, $p_{\mu}$ is contained in the 1-skeleton of the Coxeter cell complex. Using paths $p_{\mu}$ as a model, one defines generalized Hecke and LS paths: These are paths $p$ in the 1-skeleton of the Coxeter complex, where each $p$ is a concatenation of Hecke (resp. LS) paths $p_{1}, \ldots p_{\ell}$, so that

$$
\text { length }_{\Delta}\left(p_{i}\right)=\mu_{i}=c_{i} \varpi_{i} .
$$

In addition, generalized Hecke and LS paths have to satisfy certain positive-folding condition at each end-point of $p_{i}, i=1, \ldots, \ell-1$ (the positivity condition is the same for Hecke and LS paths). We refer to $\left[\mathrm{KM}_{2}\right]$ and $\left[\mathrm{GL}_{2}\right]$ for the precise definition.

We let Hecke $e_{x, y, \mu}^{1}$ and $L S_{x, y, \mu}^{1}$ denote the set of generalized Hecke and LS paths connecting $x$ to $y$. We also define sets Hecke $e_{x, y, \mu}^{1,+}$ and $L S_{x, y, \mu}^{1,+}$ of generalized Hecke and LS paths contained in $\Delta$. It is proven in $\left[\mathrm{KM}_{2}\right]$ that the set

$$
\bigcup_{y} L S_{o, y, \mu}^{1}
$$

coincides with the set of paths in $A$ obtained from $p_{\mu}$ by applying root operators. Thus, Littelmann's proof of Theorem 73 goes through in the case of generalized LS paths and we obtain

Theorem $74\left(\left[\mathrm{KM}_{2}\right]\right)$. Cardinalities of $L S_{x_{\lambda}, x_{\eta}, \mu}^{+}$and $L S_{x_{\lambda}, x_{\eta}, \mu}^{1,+}$ are the same and equal $n_{\lambda, \mu}^{\eta}$.

One of the key advantages of generalized Hecke paths is the following:

Lemma 75. If $p$ is a generalized Hecke path, then $k_{R} \cdot p$ is a generalized LS path.

Proof. Scaling by any natural number $k$ sends Hecke $\mathrm{e}_{x, y, \mu}^{1}$ to Hecke ${ }_{k x, k y, k \mu}^{1}$. Moreover, since break-points of every path $p \in \mathrm{Hecke}_{x, y, \mu}^{1}$ are at vertices of $A$, the break-points of $k \cdot p$ are at special vertices of $A$. Therefore, the path $k_{R} \cdot p$ satisfies the Maximality Axiom and, hence, is a generalized LS-path.

Remark 20. Already for $R=A_{2}$, there are Hecke paths which are not LS paths, even though $k_{R}=1$.

\section{A.7. Path model connection of triangles in buildings and tensor product multiplicities}

Pick a special vertex in $X$ which is the image of $o \in A$ under a chart; by abusing the notation we will again denote this vertex of $X$ by $o$. We then have the natural projection $\mathbb{P}_{\Delta}: X \rightarrow \Delta$,

$$
x \mapsto d_{\Delta}(o, x) .
$$

It is easy to see that $\mathbb{P}_{\Delta}$ sends each geodesic path $\widetilde{p}$ in $X$ to a piecewise-geodesic path $p$ in $\Delta$ so that

$$
\operatorname{length}_{\Delta}(p)=\text { length }_{\Delta}(\widetilde{p}) .
$$

Also, the image of every geodesic path $\widetilde{p}=o x$ under $\mathbb{P}_{\Delta}$ is again a geodesic. With a bit more care, one proves that for every oriented geodesic triangle $\tau=x y z$, so 


\section{EIGENVALUE PROBLEM}

that $x$ is a vertex of $X$, one can choose a special vertex $o \in X$ so that the paths $\mathbb{P}_{\Delta}(x y)$ and $\mathbb{P}_{\Delta}(z x)$ are still geodesic (see $\left.\left[\mathrm{KM}_{2}\right]\right)$. We will refer to such $\sigma=\mathbb{P}_{\Delta}(\tau)$ as a broken triangle.

We now can state the key results connecting geodesics in $X$ and the path model(s) in $A$ :

\section{Theorem $76\left(\left[\mathrm{KM}_{2}\right]\right)$.}

1. For every geodesic path $\widetilde{p}$ in $X$, its projection $p=\mathbb{P}_{\Delta}(\widetilde{p})$ is a Hecke path.

2. Conversely, every Hecke path $p$ in $\Delta$ is the $\mathbb{P}_{\Delta}$-projection of a geodesic path in $X$.

3. Let $\phi: A \rightarrow X$ be a chart and let $p_{\mu} \subset A$ be a model generalized Hecke path. Then $\mathbb{P}_{\Delta}\left(\phi\left(p_{\mu}\right)\right)$ is a generalized Hecke path in $\Delta$.

Remark 21. 1. Some of the arguments in $\left[\mathrm{KM}_{2}\right]$ were simplified in [GGPR].

2. In $\left[\mathrm{KM}_{2}\right]$ we could not prove that every generalized Hecke path in $\Delta$ can be unfolded to a model generalized Hecke path in an apartment in $X$. This was accomplished later on by Gaussent and Littelmann in $\left[\mathrm{GL}_{2}\right]$.

We can now apply these results to triangles in $X$. First of all, if $\sigma \subset \Delta$ is a Littelmann triangle with the side-lengths $\lambda, \mu, \nu \in \Delta$, then $\sigma$ is also a Hecke triangle. In view of Part 2 of Theorem 76, the broken triangle $\sigma$ can be unfolded to an (oriented) geodesic triangle $\tau \subset X$ whose vertices are in $\mathrm{Gr}_{G}$ and whose $\Delta$-side lengths are still $\lambda, \mu, \nu$. This gives an alternative proof of the inclusion

$$
\operatorname{Rep}\left(G^{\vee}\right) \subset \operatorname{Hecke}(G) .
$$

(The first proof was based on the Satake transform; see section A.5.)

The second corollary is a saturation theorem for the set Hecke $(G)$. Suppose that $\tau=x y z$ is an oriented geodesic triangle in $X$ (with the $\Delta$-side lengths $\lambda, \mu, \nu$ ), whose vertices are vertices of $X$. Then multiplication by $k=k_{R}$ sends the broken triangle $\sigma:=\mathbb{P}_{\Delta}(\tau)$ to a new broken triangle $\sigma^{\prime}=k \sigma$, whose vertices are special vertices of $A$. The broken side of $\sigma^{\prime}$ is still a Hecke path; thus, the new broken triangle $\sigma^{\prime}$ is a Hecke triangle in $A$. In view of Part 2 of Theorem 76, the Hecke triangle $\sigma^{\prime}$ can be unfolded to a special geodesic triangle $\tau^{\prime}$ in $X$ whose $\Delta$-side lengths are $k \lambda, k \mu, k \nu$. Furthermore:

Theorem $77\left(\left[\mathrm{KM}_{2}\right]\right)$. Suppose that $(\lambda, \mu, \nu) \in\left(L_{+}\right)^{3}$ and $\lambda+\mu+\nu \in Q\left(R^{\vee}\right)$. Then

$$
\exists N \in \mathbb{N}, N(\lambda, \mu, \nu)=\left(\lambda^{\prime}, \mu^{\prime}, \nu^{\prime}\right) \in \operatorname{Hecke}(G) \Rightarrow k_{R}(\lambda, \mu, \nu) \in \operatorname{Hecke}(G) .
$$

Proof. By assumption, there exists an oriented geodesic triangle $\tau^{\prime}$ in $X$ with $\Delta$ side lengths $\left(\lambda^{\prime}, \mu^{\prime}, \nu^{\prime}\right)$. Then every $\psi \in \operatorname{Gauss}\left(\tau^{\prime}\right)$ is semistable (Theorem 67). By the same theorem, since semistability is preserved by scaling, there exists an oriented triangle $\tau \in \mathcal{T}_{\lambda, \mu, \nu}$, whose vertices are vertices of $X$. Thus, for $k=k_{R}$, the broken triangle $\sigma^{\prime}=k\left(\mathbb{P}_{\Delta}(\tau)\right)$ is a Hecke triangle. Hence, by Theorem 76 (Part 2), this Hecke triangle can be unfolded to a geodesic triangle

Hence,

$$
\tau^{\prime \prime} \in \mathcal{T}_{k \lambda, k \mu, k \nu}^{s p} .
$$

Similarly, we obtain

$$
k(\lambda, \mu, \nu) \in \operatorname{Hecke}(G) .
$$


Theorem $78\left(\left[\mathrm{KM}_{2}\right]\right)$.

$$
k_{R} \cdot \operatorname{Hecke}(G) \subset \operatorname{Rep}\left(G^{\vee}\right) .
$$

Proof. Suppose $(\lambda, \mu, \nu) \in \operatorname{Hecke}(G)$. Take a special triangle $\tau=x y z \subset X$ with the $\Delta$-side lengths $\lambda, \mu, \nu$, where $x=o$. Next, consider an apartment $A^{\prime}=\phi(A) \subset X$ containing $y, z$ and replace the geodesic $y z$ with the model generalized Hecke path $p_{\mu}$ in $\Delta^{\prime} \subset A^{\prime}$, connecting $y$ to $z$ and having the $\Delta$-length $\mu$. Here $\Delta^{\prime} \subset A^{\prime}$ is a Weyl chamber with the tip $y$ containing the point $z$. The result is a "broken triangle" $\Pi \subset X$ (actually, $\Pi$ is a polygon but we prefer to think of the concatenation $p_{\mu}$ as a broken side of a triangle). Now, projecting $\Pi$ to $\Delta$ via $\mathbb{P}_{\Delta}$ results in a generalized Hecke triangle $\Sigma$ with two geodesic sides $x x_{\lambda}, x_{\nu} x$ as before and the broken side $\mathbb{P}_{\Delta}\left(p_{\mu}\right)$ which is a generalized Hecke path $p$. Scaling by $k=k_{R}$ sends $p$ to a generalized LS path $k \cdot p$ (see Lemma 75 ). Thus, the rescaled polygon $k \cdot \mathbb{P}_{\Delta}(\Sigma)$ is a generalized Littelmann triangle with the $\Delta$-side lengths $k \lambda, k \mu, k \nu$. Hence,

$$
n_{\lambda^{\prime}, \mu^{\prime}, \nu^{\prime}} \neq 0
$$

where $\left(\lambda^{\prime}, \mu^{\prime}, \nu^{\prime}\right)=k(\lambda, \mu, \nu)$.

\section{Corollary 79.}

1. $k_{R} \cdot \operatorname{Hecke}(G) \subset \operatorname{Rep}\left(G^{\vee}\right) \subset \operatorname{Hecke}(G)$.

2. For a root system $R$ of type $\mathrm{A}, \operatorname{Rep}\left(G^{\vee}\right)=\operatorname{Hecke}(G)$.

Theorem 76 was improved by Gaussent and Littelmann as follows:

Theorem 80 (S. Gaussent, P. Littelmann, $\left[\mathrm{GL}_{2}\right]$ ). For every generalized Hecke path $p$ in $\Delta$ with length $_{\Delta}(p)=\mu$, there exists a chart $\phi: A \rightarrow X$, so that $p=\mathbb{P}_{\Delta}\left(\phi\left(p_{\mu}\right)\right)$.

Using this result, they computed in $\left[\mathrm{GL}_{2}\right]$ the structure constants $m_{\lambda, \mu}^{\eta}$ for the spherical Hecke ring $\mathcal{H}_{G}$ using the path model based on generalized Hecke paths simplifying the earlier work by C. Schwer [Sc].

\section{A.8. Saturation Theorems and conjectures}

Recall that semigroup $\operatorname{Rep}\left(G^{\vee}\right)$ is contained in the eigencone $C=C\left(G^{\vee}\right)$ which is the set of positive real linear combinations of elements of $\operatorname{Rep}\left(G^{\vee}\right)$. In particular,

$$
(\lambda, \mu, \nu) \in\left(L_{+}\right)^{3} \cap C \Longleftrightarrow \exists N>0,(N \lambda, N \mu, N \nu) \in \operatorname{Rep}\left(G^{\vee}\right) .
$$

We define the semigroup

$$
C_{L}=C \cap\left(L_{+}\right)^{3} \cap\left\{\lambda+\mu+\nu \in Q\left(R^{\vee}\right)\right\}
$$

containing $\operatorname{Rep}\left(G^{\vee}\right)$. Recall also that

$$
\operatorname{Hecke}(G)=\left\{(\lambda, \mu, \nu): \widehat{\mathcal{T}}_{\lambda, \mu, \nu}^{s p} \neq \varnothing\right\} \subset \operatorname{Tri}(X)=\left\{(\lambda, \mu, \nu): \widehat{\mathcal{T}}_{\lambda, \mu, \nu} \neq \varnothing\right\} .
$$

In view of Theorem 67 , the set $\operatorname{Tri}(X)$ is stable under scaling (since scaling preserves semistability). 
Theorem $81\left(\left[\mathrm{KLM}_{3}, \mathrm{KM}_{2}\right]\right)$.

(1) $k_{R} C_{L} \subset \operatorname{Hecke}(G) \subset C_{L}$.

(2) $k_{R} \operatorname{Hecke}(G) \subset \operatorname{Rep}(G) \subset \operatorname{Hecke}(G)$.

(3) $k_{R}^{2} C_{L} \subset \operatorname{Rep}\left(G^{\vee}\right)$.

Proof. The only result that we did not yet explain is the inclusion Hecke $(G) \subset C_{L}$. Note that

$$
k_{R} \operatorname{Hecke}(G) \subset \operatorname{Rep}(G) \subset C_{L} .
$$

Since $C$ is a cone,

$$
\operatorname{Hecke}(G) \subset \frac{1}{k_{R}} C_{L} \subset C \text {. }
$$

On the other hand,

$$
\operatorname{Hecke}(G) \subset L^{3} \cap\left\{\lambda+\mu+\nu \in Q\left(R^{\vee}\right)\right\} .
$$

The inclusions (1) in this theorem are strengthened to

Theorem $82\left(\left[\mathrm{KLM}_{1}\right]\right) \cdot C\left(G^{\vee}\right)=\operatorname{Tri}(X)$.

Proof. This theorem is proven in $\left[\mathrm{KLM}_{1}\right]$ by direct geometric arguments; here we will present an indirect partial proof using the results that we explained so far. Let us first verify the inclusion $C \cap(\mathbb{Q} \otimes L)^{3} \subset \operatorname{Tri}(X)$. Since both sets are stable under rational scaling, it suffices to consider a triple $(\lambda, \mu, \nu) \in N \cdot C_{L}$ for large $N$, i.e.,

$$
(\lambda, \mu, \nu) \in \operatorname{Rep}\left(G^{\vee}\right) \subset \operatorname{Hecke}(G) \subset \operatorname{Tri}(X) .
$$

Note, furthermore, that in view of the local compactness of $X$ (or, by appealing to projections of triangles in $X$ to $\Delta$ ), the set $\operatorname{Tri}(X)$ is closed. Since rational triples are dense in $C$, we obtain the inclusion

$$
C \subset \operatorname{Tri}(X) .
$$

In the same fashion one proves that

$$
\operatorname{Tri}(X) \cap(L \otimes \mathbb{Q})^{3} \subset C .
$$

One can finish a proof by arguing that rational points are dense in $\operatorname{Tri}(X)$. This, of course, follows from the results of $\left[\mathrm{KLM}_{1}\right]$, where it is proven that $\operatorname{Tri}(X)$ is a rational cone. One can also give an alternative argument using root operators acting on generalized LS paths following the arguments used in $\left[\mathrm{KM}_{2}\right]$.

As a corollary, we obtain:

Theorem 83 (Saturation Theorem, $\left.\left[\mathrm{KM}_{2}\right]\right)$. If $\lambda+\mu+\nu \in Q\left(R^{\vee}\right)$ and $\lambda, \mu, \nu$ are dominant weights of $G^{\vee}$ such that

$$
\exists N>0 \quad\left(V_{N \lambda} \otimes V_{N \mu} \otimes V_{N \nu}\right)^{G^{\vee}} \neq 0
$$

then

$$
\left(V_{k \lambda} \otimes V_{k \mu} \otimes V_{k \nu}\right)^{G^{\vee}} \neq 0
$$

for $k=k_{R}^{2}$. In particular, for $R=A_{\ell}, k_{R}=1$ and we recover the Saturation Theorem of Knutson and Tao [KT]. 


\section{SHRAWAN KUMAR}

Proof. This theorem follows immediately from the combination of the results in Theorem 81. It is useful, however, to sketch the overall argument. Let $N>0$ be such that

$$
(N \lambda, N \mu, N \nu) \in \operatorname{Rep}\left(G^{\vee}\right) .
$$

By the inclusion

$$
\operatorname{Rep}\left(G^{\vee}\right) \subset \operatorname{Hecke}(G)
$$

(proven either using Satake correspondence as in section A.5 or via LS path model as in section A.7),

$$
(N \lambda, N \mu, N \nu) \in \operatorname{Hecke}(G) .
$$

Let $\tau$ be a special oriented triangle in $X$ with the $\Delta$-side lengths $(N \lambda, N \mu, N \nu)$. Then, by Theorem 67 , every weighted configuration

$$
\psi \in \operatorname{Gauss}(\tau)
$$

is semistable. Since semistability is preserved by scaling, the weighted configuration $\frac{1}{N} \psi$ is still stable. Thus, by Theorem 67 , there exists an oriented triangle in $X$ with the $\Delta$-side lengths $(N \lambda, N \mu, N \nu)$, whose vertices are vertices of $X$, in view of the condition

$$
\lambda+\mu+\nu \in \mathbb{Q}\left(R^{\vee}\right) .
$$

Therefore, by Theorem 77,

$$
\left(\lambda^{\prime}, \mu^{\prime}, \nu^{\prime}\right)=k_{R}(\lambda, \mu, \nu) \in \operatorname{Hecke}(G) .
$$

Lastly, by Theorem 78 ,

$$
k_{R}^{2}(\lambda, \mu, \nu)=k_{R}\left(\lambda^{\prime}, \mu^{\prime}, \nu^{\prime}\right) \in \operatorname{Rep}\left(G^{\vee}\right) .
$$

It is, then, natural to ask to what extent the "saturation factors" $k_{R}$ and $k_{R}^{2}$ are needed in the above results.

Theorem 84. Let $R$ be a non-simply laced root system. Then there are triples $(\lambda, \mu, \nu)$ so that

$$
(\lambda, \mu, \nu) \in C_{L}
$$

but

$$
(\lambda, \mu, \nu) \notin \operatorname{Rep}\left(G^{\vee}\right)
$$

i.e.,

$$
\left(V_{\lambda} \otimes V_{\mu} \otimes V_{\nu}\right)^{G^{\vee}}=0 .
$$

Moreover, in these examples, the triple $(\lambda, \mu, \nu)$ belongs to $\operatorname{Hecke}(G)$. 


\section{EIGENVALUE PROBLEM}

Proof. It is convenient to switch now the notation from $R^{\vee}$ to $R$ and from $G^{\vee}$ to $G$.

In $\left[\mathrm{KLM}_{3}\right]$ we constructed examples of such triples for $R=\mathrm{B}_{2}=\mathrm{C}_{2}$ and $R=\mathrm{G}_{2}$. Below we will explain how to generalize these examples to the case $R=\mathrm{F}_{4}$, $R=\mathrm{C}_{\ell}, R=\mathrm{B}_{\ell}$. In each case, we will use a triple of weights $(\lambda, \lambda, \lambda)$, where $\lambda$ is one of the fundamental weights. In all cases we will choose $\lambda$ which belongs to the root lattice, and, hence, the condition

$$
3 \lambda \in Q(R)
$$

(necessary for $(\lambda, \lambda, \lambda) \in$ Hecke) is trivially satisfied. Thus, $(\lambda, \lambda, \lambda)$ belongs to Hecke; see Appendix to $\left[\mathrm{H}_{2}\right]$.

We now specify the weight $\lambda$ :

(1) For $R=F_{4}$ we take $\lambda=\varpi_{2}$ (note that $\varpi_{3}$ does not give an example as $\left.\left(\varpi_{3}, \varpi_{3}, \varpi_{3}\right) \in \operatorname{Rep}\left(F_{4}\right)\right)$. The proof in this case is an unilluminating computation using the LiE program for tensor product decomposition.

(2) For $R=C_{2 m}$ we take $\lambda=\varpi_{\ell}$ (the longest fundamental weight), while for $R=C_{2 m+1}$ we will take $\lambda=\varpi_{\ell-1}$ (the next to the longest fundamental weight).

(3) For $R=B_{\ell}, \ell>2$, we take $\lambda=\varpi_{1}$.

Note that for the root systems of type B and C, we have chosen $\lambda$ so that for the point $x=x_{\lambda}$, the interior of the segment $o x$ intersects affine walls in exactly one point (the midpoint). We will give a proof that $(\lambda, \lambda, \lambda) \notin \operatorname{Rep}$ for $R=\mathrm{C}_{\ell}$ since the $B_{\ell}$ case is done by the same method.

Consider first the case when $\ell$ is even. Then

$$
\lambda=(1, \ldots, 1)
$$

in the Bourbaki coordinates. Suppose that $(\lambda, \lambda, \lambda) \in \operatorname{Rep}(\operatorname{Sp}(\ell))$. Let $x=x_{\lambda}=$ $(1, \ldots, 1)$. Since the interior of the segment $o x$ intersects only one wall, every (positive) LS path $p$ connecting $x$ to itself has exactly one break point, a point $y \in \Delta$. Set $\mu=\overrightarrow{y x}$. Then

$$
\mu \in W \cdot \frac{1}{2} \lambda \text {. }
$$

Since $y \in \Delta$, we conclude that

$$
y=\left(\frac{3}{2}, \ldots, \frac{3}{2}, \frac{1}{2}, \ldots, \frac{1}{2}\right) .
$$

The path $p$ is the concatenation of the segments $x y$ and $y x$. We claim that unless $y=(1 / 2, \ldots, 1 / 2)$, the path $p$ is not a Hecke path. Indeed, in order for $p$ to be a Hecke path we would need at least

$$
\overrightarrow{x y} \leq_{\Delta^{*}} \overrightarrow{y x} \Longleftrightarrow 2 \mu=(-1, \ldots,-1,1, \ldots, 1) \in \Delta^{*} .
$$

The latter is clearly false, unless the vector $-2 \mu$ has only positive coordinates, i.e., $y=\frac{1}{2} x$. In the latter case, however, the path $p$ is Hecke but not LS, analogously to $\left[\mathrm{KM}_{1}\right]$ : The Maximality Axiom is violated. 


\section{SHRAWAN KUMAR}

Suppose now that $\ell$ is odd. Then

$$
\lambda=(1, \ldots, 1,0) .
$$

Again, every positive LS path connecting $x$ to itself has exactly one break, at a point $y \in \Delta$. Thus, the point $y$ has to be of the form

$$
\left(\frac{3}{2}, \ldots, \frac{3}{2}, \frac{1}{2}, \ldots, \frac{1}{2}, 0\right)
$$

(as the last coordinate has to be nonnegative). Now, the argument is exactly the same as in the even case.

On the other hand, all known examples fail for simply-laced groups. Furthermore, in all known examples, at least one weight is singular.

Conjecture 85 (Saturation Conjecture).

1. If $R$ is a simply-laced root system, then

$$
(\lambda, \mu, \nu) \in C_{L} \Longleftrightarrow\left(V_{\lambda} \otimes V_{\mu} \otimes V_{\nu}\right)^{G^{\vee}} \neq 0 .
$$

2. In general,

$$
(\lambda, \mu, \nu) \in C_{L} \Rightarrow\left(V_{2 \lambda} \otimes V_{2 \mu} \otimes V_{2 \nu}\right)^{G^{\vee}} \neq 0 .
$$

3. If $\lambda, \mu, \nu$ are regular weights then

$$
(\lambda, \mu, \nu) \in C_{L} \Longleftrightarrow\left(V_{\lambda} \otimes V_{\mu} \otimes V_{\nu}\right)^{G^{\vee}} \neq 0 .
$$

We refer the reader to $\left[\mathrm{KM}_{1}\right]$ for more detailed discussion of the semigroup $\operatorname{Rep}\left(G^{\vee}\right)$ and the set Hecke $(G)$.

\section{References}

[Bll] W. Ballmann, Lectures on Spaces of Nonpositive Curvature (With an Appendix by Misha Brin), DMV Seminar, Vol. 25, Birkhäuser Verlag, Basel, 1995.

[Bls] A. Balser, Polygons with prescribed Gauss map in Hadamard spaces and Euclidean buildings, Canad. Math. Bull. 49 (2006), 321-336.

[B 1 P. Belkale, Local systems on $\mathbb{P}^{1}-S$ for $S$ a finite set, Compositio Math. 129 (2001), 67-86.

$\left[\mathrm{B}_{2}\right] \quad$ P. Belkale, Invariant theory of $G L(n)$ and intersection theory of Grassmannians, IMRN 2004, no. 69, 3709-3721.

$\left[\mathrm{B}_{3}\right] \quad$ P. Belkale, Geometric proofs of Horn and saturation conjectures, J. Alg. Geom. 15 (2006), 133-173.

[B 4 P. Belkale, Geometric proof of a conjecture of Fulton, Advances Math. 216 (2007), 346-357.

[BK 1 P. Belkale, S. Kumar, Eigenvalue problem and a new product in cohomology of flag varieties, Invent. Math. 166 (2006), 185-228. 


\section{EIGENVALUE PROBLEM}

$\left[\mathrm{BK}_{2}\right] \quad$ P. Belkale, S. Kumar, Eigencone, saturation and Horn problems for symplectic and odd orthogonal groups, J. Algebraic Geom. 19 (2010), 199-242.

[BKR] P. Belkale, S. Kumar, N. Ressayre, A generalization of Fulton's conjecture for arbitrary groups, Math. Annalen 354 (2012), 401-425.

[BeKa] A. Berenstein, M. Kapovich, Stability inequalities and universal Schubert calculus of rank 2, Transformation Groups 16 (2011), 955-1007.

[BS] A. Berenstein, R. Sjamaar, Coadjoint orbits, moment polytopes, and the HilbertMumford criterion, Journ. Amer. Math. Soc. 13 (2000), 433-466.

[BL] S. Billey, V. Lakshmibai, Singular Loci of Schubert Varieties, Progress in Mathematics, Vol. 182, Birkhäuser, Boston, 2000.

[Bo] N. Bourbaki, Groupes et Algèbres de Lie, Chap. 4-6, Masson, Paris, 1981.

[Bra] E. Braley, Eigencone Problems for Odd and Even Orthogonal Groups, PhD thesis (under the supervision of P. Belkale), University of North Carolina, 2012.

[Br] M. Brion, Restriction de represéntations et projections d'orbites coadjointes (d'apres Belkale, Kumar et Ressayre), Séminaire Bourbaki, 64ème année, Vol. 2011/2012, no. 1043, Soc. Math. de France, pp. 2011-2012.

[BD] T. Brocker, T. tom Dieck, Representations of Compact Lie Groups, Graduate Texts in Mathematics, Vol. 98, Springer-Verlag, Berlin, 1985.

[BT] F. Bruhat, J. Tits, Groupes reductifs sur un corps local, Inst. Hautes Études Sci. Publ. Math. 41 (1972), 5-251.

[CG] N. Chriss, V. Ginzburg, Representation Theory and Complex Geometry, Birkhäuser, Boston, 1997.

[DK] В. И. Данилов, Г. А. Кошевой, Дискретная выпуклость и эрмитовы матрицы, Труды мат. инст. им. В. А. Стеклова 241 (2003), 68-89. Engl. transl.: V. I. Danilov, G. A. Koshevoi, Discrete convexity and Hermitian matrices, Proc. Steklov Inst. Math. 241 (2003), 58-78.

[DW] H. Derksen, J. Weyman, Semi-invariants of quivers and saturation for Littlewood-Richardson coefficients, J. Amer. Math. Soc. 13 (2000), 467-479.

$\left[\mathrm{DR}_{1}\right] \quad$ I. Dimitrov, M. Roth, Cup products of line bundles on homogeneous varieties and generalized PRV components of multiplicity one, preprint (2009).

$\left[\mathrm{DR}_{2}\right]$ I. Dimitrov, M. Roth, Geometric realization of $P R V$ components and the Littlewood-Richardson cone, Contemp. Math. 490 (2009), Amer. Math. Soc., Providence, RI, 83-95.

[DH] I. Dolgachev, Y. Hu, Variations of geometric invariant theory quotients, Publ. Math. IHES 87 (1998), 5-51.

[D 1 H. Duan, The degree of a Schubert variety, Advances in Math. 180 (2003), 112-133.

$\left[\mathrm{D}_{2}\right] \quad \mathrm{H}$. Duan, Multiplicative rule of Schubert classes, Invent. Math. 159 (2005), 407-436.

[DZ] H. Duan, X. Zhao, Algorithm for multiplying Schubert classes, Internat. J. Algebra Comput. 16 (2006), 1197-1210.

[EH] D. Eisenbud, J. Harris, Divisors on general curves and cuspidal rational curves, Invent. Math. 74 (1983), 371-418. 


\section{SHRAWAN KUMAR}

[E] A. G. Elashvili, Invariant algebras, in: "Lie Groups, their Discrete Subgroups, and Invariant Theory" (ed. E. B. Vinberg), Advances in Soviet Math. 8, Amer. Math. Soc., Providence, 1992, pp. 57-64.

[EG $\mathrm{EG}_{1}$ S. Evens, W. Graham, The Belkale-Kumar cup product and relative Lie algebra cohomology. With an appendix by S. Evens, W. Graham and E. Richmond, Int. Math. Res. Not. IMRN 8 (2013), 1901-1933.

$\left[\mathrm{EG}_{2}\right] \mathrm{S}$. Evens, W. Graham, The relative Hochschild-Serre spectral sequence and the Belkale-Kumar product, Trans. Amer. Math. Soc. 365 (2013), no. 11, 58335857.

[Fa] K. Fan, On a theorem of Weyl concerning eigenvalues of linear transformations, invariant factors, highest weights, and Schubert calculus, Proc. Natl. Acad. Sci. USA 35 (1949), 652-655.

[FK] B. Fontaine, J. Kamnitzer, Cyclic sieving, rotation, and geometric representation theory, Selecta Math. (N.S.) 20 (2014), no. 2, 609-625.

[FKK] B. Fontaine, J. Kamnitzer, G. Kuperberg, Buildings, spiders, and geometric Satake, Compositio Math. 149 (2013), no. 11, 1871-1912.

$\left[\mathrm{F}_{0}\right]$ W. Fulton, Young Tableaux, London Math. Society, Cambridge University Press, 1997.

[F1] W. Fulton, Intersection Theory, 2nd edn., Springer, New York, 1998.

$\left[\mathrm{F}_{2}\right]$ W. Fulton, Eigenvalues, invariant factors, highest weights, and Schubert calculus, Bull. Amer. Math. Soc. (N.S.) 37 (2000), 209-249.

[G] C. F. Gauss, Letter to W. Bolyai, Collected Works, Vol. 8, 222-223.

[GGPR] S. Gaussent, C. Charignon, N. Bardy-Panse, G. Rousseau, Une preuve plus immobiliŕe du thórème de "saturation" de Kapovich-Leeb-Millson, Enseign. Math. (2) 59 (2013), no. 1-2, 3-37.

$\left[\mathrm{GL}_{1}\right] \quad \mathrm{S}$. Gaussent, P. Littelmann, LS-Galleries, the path model and $M V$-cycles, Duke Math. J. 127 (2005), 35-88.

$\left[\mathrm{GL}_{2}\right] \quad \mathrm{S}$. Gaussent, P. Littelmann, One-skeleton galleries, the path model and a generalization of Macdonald's formula for Hall-Littlewood polynomials, Int. Math. Res. Notices 12 (2012), 2649-2707.

[GR] S. Gaussent, G. Rousseau, Kac-Moody groups, hovels and Littelmann's paths, Annales Inst. Fourier 58 (2008), 2605-2657.

[Gro] B. Gross, On the Satake isomorphism, in: Galois Representations in Arithmetic Algebraic Geometry (Durham, 1996), London Math. Soc. Lecture Notes, Vol. 254, Cambridge Univ. Press, Cambridge, 1998, pp. 223-237.

$\left[\mathrm{H}_{1}\right] \quad$ T. Haines, Structure constants for Hecke and representations rings, IMRN 39 (2003), 2103-2119.

$\left[\mathrm{H}_{2}\right]$ T. Haines, Equidimensionality of convolution morphisms and applications to saturation problems (with Appendix by T. Haines, M. Kapovich, J. J. Millson), Advances in Math. 20 (2006), 297-327.

[HKM] T. Haines, M. Kapovich, J. J. Millson, Ideal quadrilaterals in Euclidean buildings, constant term maps for spherical Hecke rings and branching to Levi subgroups, J. Algebra 361 (2012), 41-78.

[H] R. Hartshorne, Algebraic Geometry, Springer-Verlag, New York, 1977. Russian transl.: P. Хартсхорн, Алгебрачческая геометрия, Мир, М., 1981. 


\section{EIGENVALUE PROBLEM}

[He] W. H. Hesselink, Desingularizations of varieties of nullforms, Invent. Math. 55 (1979), 141-163.

[Ho] A. Horn, Eigenvalues of sums of Hermitian matrices, Pacific J. Math. 12 (1962), 225-241.

[J] J. C. Jantzen, Representations of Algebraic Groups, 2nd edn., Mathematical Surveys and Monographs, Vol. 107, Amer. Math. Soc., Providence, RI, 2003.

[Kam] J. Kamnitzer, Mirkovic-Vilonen cycles and polytopes, Annals of Math. (2) 171 (2010), 245-294.

[Kap] M. Kapovich, Generalized triangle inequalities and their applications, Proceedings of the International Congress of Mathematicians, Madrid, (2006), 719-742.

[KKM] M. Kapovich, S. Kumar, J. J. Millson, The eigencone and saturation for Spin(8), Pure and Applied Math. Quarterly 5 (2009), 755-780.

$\left[\mathrm{KLM}_{1}\right]$ M. Kapovich, B. Leeb, J. J. Millson, Convex functions on symmetric spaces, side lengths of polygons and the stability inequalities for weighted configurations at infinity, J. Diff. Geom. 81 (2009), 297-354.

$\left[\mathrm{KLM}_{2}\right]$ M. Kapovich, B. Leeb, J. J. Millson, Polygons in buildings and their refined side-lengths, Geom. Analysis and Funct. Analysis 19 (2009), 1081-1100.

$\left[\mathrm{KLM}_{3}\right]$ M. Kapovich, B. Leeb, J. J. Millson, The Generalized Triangle Inequalities in Symmetric Spaces and Buildings with Applications to Algebra, Memoirs of AMS, Vol. 192, Amer. Math. Soc., Providence, RI, 2008.

[KL] B. Kleiner, B. Leeb, Rigidity of quasi-isometries for symmetric spaces and Euclidean buildings, Publ. Math. IHES 86 (1997), 115-197.

$\left[\mathrm{KM}_{1}\right] \quad$ M. Kapovich, J. J. Millson, Structure of the tensor product semigroup, Asian J. Math. 10 (2006), 492-540.

$\left[\mathrm{KM}_{2}\right]$ M. Kapovich, J. J. Millson, A path model for geodesics in Euclidean buildings and its applications to representation theory, Groups, Geometry and Dynamics 2 (2008), 405-480.

[Ki] F. Kirwan, Cohomology of Quotients in Symplectic and Algebraic Geometry, Princeton University Press, 1984.

[Kly] A. Klyachko, Stable bundles, representation theory and Hermitian operators, Selecta Mathematica 4 (1998), 419-445.

[KP] A. Knutson, K. Purbhoo, Product and puzzle formulae for $G L_{n}$ Belkale-Kumar coefficients, Electr. J. Combinatorics 18 (2011), P76.

[KT] A. Knutson, T. Tao, The honeycomb model of $G L_{n}(\mathbb{C})$ tensor products I: Proof of the saturation conjecture, J. Amer. Math. Soc. 12 (1999), 1055-1090.

$[\mathrm{KTW}]$ A. Knutson, T. Tao, C. Woodward, The honeycomb model of $G L_{n}(\mathbb{C})$ tensor products II: Puzzles determine facets of the Littlewood-Richardson cone, J. Amer. Math. Soc. 17 (2004), 19-48.

[Ko] B. Kostant. Lie algebra cohomology and the generalized Borel-Weil theorem, Annals of Math. 74 (1961), 329-387.

$\left[\mathrm{K}_{1}\right] \quad$ S. Kumar, Kac-Moody Groups, their Flag Varieties and Representation Theory, Progress in Mathematics, Vol. 204, Birkhäuser, Boston, 2002.

$\left[\mathrm{K}_{2}\right] \quad$ S. Kumar, On the Cachazo-Douglas-Seiberg-Witten conjecture for simple Lie algebras, J. Amer. Math. Soc. 21 (2008), 797-808. 


\section{SHRAWAN KUMAR}

$\left[\mathrm{K}_{3}\right] \quad$ S. Kumar, Tensor product decomposition, Proc. of the International Congress of Mathematicians, Hyderabad (India), (2010), 1226-1261.

[KuLM] S. Kumar, B. Leeb, J. J. Millson, The generalized triangle inequalities for rank 3 symmetric spaces of noncompact type, Contemp. Math. 332 (2003), 171-195.

[KS] S. Kumar, J. Stembridge, Special isogenies and tensor product multiplicities, Inter. Math. Res. Not. 2007 (2007), no. 20, 1-13.

[Le] B. Lee, A Comparison of Eigencones Under Certain Diagram Automorphisms, $\mathrm{PhD}$ thesis (under the supervision of S. Kumar), University of North Carolina, 2012.

[Li] В. Б. Лидский, Собственные значения суммы и произведения симметрических матрии, ДАН СССР 74 (1950), 769-772. Engl. transl.: V. B. Lidskii, The proper values of the sum and product of symmetric matrices, translated by C. D. Benster, N.B.S. Rep. 2248. U. S. Department of Commerce, National Bureau of Standards, Washington, D.C. (1953). 8 pp.

$\left[\mathrm{L}_{1}\right] \quad$ P. Littelmann, A Littlewood-Richardson rule for symmetrizable Kac-Moody algebras, Invent. Math. 116 (1994), 329-346.

$\left[\mathrm{L}_{2}\right] \quad$ P. Littelmann, Paths and root operators in representation theory, Ann. of Math. (2) 142 (1995), 499-525.

$\left[\mathrm{L}_{3}\right] \quad$ P. Littelmann, Characters of representations and paths in $\mathfrak{h}_{\mathbb{R}}^{*}$, in: Representation Theory and Automorphic Forms (Edinburgh, 1996), Proc. Sympos. Pure Math., Vol. 61, Amer. Math. Soc., Providence, RI, 1997, pp. 29-49.

[MV] I. Mirkovic, K. Vilonen, Geometric Langlands duality and representations of algebraic groups over commutative rings, Ann. of Math. (2) 166 (2007), 95143.

$\left[\mathrm{MPR}_{1}\right]$ P-L. Montagard, B. Pasquier, N. Ressayre, Two generalisations of the PRV conjecture, Compositio Math. 147 (2011), 1321-1336.

$\left[\mathrm{MPR}_{2}\right]$ P-L. Montagard, B. Pasquier, N. Ressayre, Generalisations of the PRV conjecture, II, preprint (2012).

[MR] P-L. Montagard, N. Ressayre, Sur des faces du LR-cone généralisé, Bull. SMF 135 (2007), 343-365.

[MTV] E. Mukhin, V. Tarasov, A. Varchenko, Schubert calculus and representations of the general linear group, J. Amer. Math. Soc. 22 (2009), 909-940.

[MFK] D. Mumford, J. Fogarty, F. Kirwan, Geometric Invariant Theory, 3rd edn., Ergebnisse der Mathematik und ihrer Grenzgebiete, Vol. 34, Springer, Berlin, 1994.

[N] L. Ness, A stratification of the null cone via the moment map (with an appendix by D. Mumford), Amer. J. Math. 106 (1984), 1281-1329.

[Pa] A. Parreau, Immeubles affines: construction par les normes et étude des isométries, in: Crystallographic Groups and Their Generalizations (Kortrijk, 1999), Contemp. Math., Vol. 262, Amer. Math. Soc., Providence, RI, 2000, pp. 263-302.

[PR] B. Pasquier, N. Ressayre, The saturation property for branching rulesExamples, Exp. Math. 22 (2013), no. 3, 299-312.

[P] K. Purbhoo, Vanishing and nonvanishing criteria in Schubert calculus, Inter. Math. Res. Not. (2006), Art. ID 24590, 38 pp. 


\section{EIGENVALUE PROBLEM}

[PS] K. Purbhoo, F. Sottile, The recursive nature of the cominuscule Schubert calculus, Advances Math. 217 (2008), 1962-2004.

[RR] S. Ramanan, A. Ramanathan, Some remarks on the instability flag, Tohoku Math. J. 36 (1984), 269-291.

$\left[\mathrm{R}_{0}\right] \quad \mathrm{N}$. Ressayre, The GIT equivalence for G-line bundles, Geom. Dedicata 81 (2000), 295-324.

$\left[\mathrm{R}_{1}\right] \quad \mathrm{N}$. Ressayre, Geometric invariant theory and the generalized eigenvalue problem, Invent. Math. 180 (2010), 389-441.

$\left[\mathrm{R}_{2}\right] \quad \mathrm{N}$. Ressayre, A short geometric proof of a conjecture of Fulton, L'Enseign. Math. 57 (2011), 103-115.

$\left[\mathrm{R}_{3}\right] \quad \mathrm{N}$. Ressayre, $A$ cohomology free description of eigencones in type $A, B$ and $C$, Inter. Math. Res. Not. (2012), no. 21, 4966-5005.

$\left[\mathrm{R}_{4}\right] \quad$ N. Ressayre, GIT-cones and quivers, Math. Zeit. 270 (2012), no. 1-2, 263-275.

$\left[\mathrm{R}_{5}\right] \quad \mathrm{N}$. Ressayre, Geometric invariant theory and generalized eigenvalue problem II, Annales de l'Institut Fourier 61 (2011), no. 4, 1467-1491.

$\left[\mathrm{R}_{6}\right] \quad \mathrm{N}$. Ressayre, Multiplicative formulas in Cohomology of $G / P$ and in quiver representations, Indag. Math. 22 (2011), 87-102.

$\left[\mathrm{R}_{7}\right] \quad$ N. Ressayre, Reductions for branching coefficients, preprint (2012).

[ReR] N. Ressayre, E. Richmond, Branching Schubert calculus and the Belkale-Kumar product on cohomology, Proc. Amer. Math. Soc. 139 (2011), 835-848.

[Ri $\mathrm{R}_{1} \quad$ E. Richmond, A partial Horn recursion in the cohomology of flag varieties, J. Algebraic Comb. 30 (2009), 1-17.

$\left[\mathrm{Ri}_{2}\right] \quad$ E. Richmond, A multiplicative formula for structure constants in the cohomology of flag varieties, Michigan Math. J. 61 (2012), 3-17.

[R] M. Ronan, Lectures on Buildings, Perspectives in Mathematics , Vol. 7, Academic Press, New York, 1989.

[Ro] M. Roth, Reduction rules for Littlewood-Richardson coefficients, Inter. Math. Res. Not. (2011), no. 18, 4105-4134.

[Sj] R. Sjamaar, Convexity properties of the moment mapping re-examined, Advances Math. 138 (1998), 46-91.

[S] S. Sam, Symmetric quivers, invariant theory, and saturation theorems for the classical groups, Adv. Math. 229 (2012), 1104-1135.

[Sc] C. Schwer, Galleries, Hall-Littlewood polynomials and structure constants of the spherical Hecke algebra, Int. Math. Res. Notes (2006) Art. ID 75395, 31 pp.

[Sh] И. Р. Шафаревич, Основы алгебраической геометрии, Наука, М., 1972. Engl. transl.: I. R. Shafarevich, Basic Algebraic Geometry 1, Springer-Verlag, Berlin, 1977.

[So] F. Sottile, General isotropic flags are general (for Grassmannian Schubert calculus ), J. Algebraic Geom. 19 (2010), 367-370.

[TF] R.C. Thompson, L. Freede, On the eigenvalues of a sum of Hermitian matrices, Linear Algebra Appl. 4 (1971), 369-371.

[W] H. Weyl, Das asymptotische verteilungsgesetz eigenwerte lineare partieller differential-gleichungen, Math. Annalen 71 (1912), 441-479. 


\section{SHRAWAN KUMAR}

[Wi] H. Wielandt, An extremum property of sums of eigenvalues, Proc. Amer. Math. Soc. 6 (1955), 106-110.

[Z] A. Zelevinsky, Littlewood-Richardson semigroups, in: New Perspectives in Algebraic Combinatorics, MSRI Publ. 38 (1999), Cambridge Univ. Press, Cambridge, 337-345. 